University of Tennessee Health Science Center

UTHSC Digital Commons

\title{
Efficacy of Temporary Fixed Retention Following Comprehensive Orthodontic Treatment
}

Mark J. Owens

University of Tennessee Health Science Center

Follow this and additional works at: https://dc.uthsc.edu/dissertations

Part of the Orthodontics and Orthodontology Commons

\section{Recommended Citation}

Owens, Mark J. , "Efficacy of Temporary Fixed Retention Following Comprehensive Orthodontic Treatment" (2011). Theses and Dissertations (ETD). Paper 191. http://dx.doi.org/10.21007/ etd.cghs.2011.0233.

This Thesis is brought to you for free and open access by the College of Graduate Health Sciences at UTHSC Digital Commons. It has been accepted for inclusion in Theses and Dissertations (ETD) by an authorized administrator of UTHSC Digital Commons. For more information, please contact jwelch30@uthsc.edu. 


\title{
Efficacy of Temporary Fixed Retention Following Comprehensive Orthodontic Treatment
}

\author{
Abstract \\ Instability of the occlusion is a common problem following orthodontic treatment. The purpose of the \\ present research was to evaluate posttreatment relapse at long-term recall ( $>10$ years) in cases $(A)$ \\ treated with temporary fixed mandibular retention plus Hawley retainers compared to (B) a similar group \\ retained with just removable retention (standard Hawley type retainers alone). Fixed retainers were \\ removed after having been in place for about 2.5 years. Following this period, use of removable retention \\ was left up to the discretion of the patient in both groups. Data consisted of orthodontic records of 166 \\ American whites, all of whom had received comprehensive orthodontic treatment, collected from multiple \\ private practice orthodontists using conventional Edgewise mechanics. Records were analyzed at \\ pretreatment, end of treatment, and at long-term posttreatment recall examination (mean $=16$ years after \\ treatment). The mandibular Incisor Irregularity index at recall averaged $1.9 \mathrm{~mm}$ in the fixed group and 2.5 \\ $\mathrm{mm}$ in the Hawley-only group. This $1 / 2-\mathrm{mm}$ difference is marginally significant statistically $(P=0.03)$, but \\ seems trivially small clinically. A $1.0 \mathrm{~mm}$ change in Incisor Irregularity during treatment was shown to be \\ associated with $0.13 \mathrm{~mm}$ of relapse long term. Mandibular intercanine width increased (mean $=1.2 \mathrm{~mm}$ ) \\ during treatment, but decreased by almost the same amount by the recall examination. Maxillary and \\ mandibular arch widths became slightly narrower after treatment. Overbite and overjet both increased \\ after treatment. Results of the present study seem more stable than those reported in most long-term \\ studies; however, there is little clinical support for a fixed-retention phase given the modest $(1 / 2-\mathrm{mm})$ \\ difference in Incisor Irregularity.

\section{Document Type} \\ Thesis

\section{Degree Name} \\ Master of Dental Science (MDS)

\section{Program} \\ Orthodontics

\section{Research Advisor} \\ Edward F. Harris, Ph.D.

\section{Keywords} \\ fixed, orthodontics, relapse, removable, retention, stability

\section{Subject Categories} \\ Dentistry | Medicine and Health Sciences | Orthodontics and Orthodontology
}




\title{
EFFICACY OF TEMPORARY FIXED RETENTION FOLLOWING COMPREHENSIVE ORTHODONTIC TREATMENT
}

\author{
A Thesis \\ Presented for \\ The Graduate Studies Council \\ The University of Tennessee \\ Health Science Center
}

\author{
In Partial Fulfillment \\ Of the Requirements for the Degree \\ Master of Dental Science \\ From The University of Tennessee
}

By

Mark J. Owens, D.D.S.

May 2011 
Copyright ( 2011 by Mark J. Owens.

All rights reserved. 


\section{DEDICATION}

This thesis is dedicated to my gracious wife, Kristy Fisher Owens, who, throughout this entire three year ordeal, has been completely supportive and never once complained about the sacrifices we had to make in order for me to return to school and complete this orthodontic program. My achieving this Master's degree in Orthodontics is as much the result of her love and support at home as it was my own effort in school. Thanks, I love you. 


\section{ACKNOWLEDGEMENTS}

There are several folks that I need to thank for their help, guidance and patience in completing this thesis project. First, I am forever thankful for an understanding wife, whose unwavering support and constant encouragement have never wavered throughout this endeavor, or our marriage. I am truly blessed to have three wonderful children, Mark, Luke, and Rhett. You'll never know how much I look forward to coming home to you guys every day and playing baseball in the yard or wrestling on "momma's bed."

I can't thank Dr. Edward F. Harris enough for all of his hard work and assistance on this project. Though rarely recognized, Dr. Harris is such a valuable asset to this department and was integral in the composition and completion of this Master's thesis. Without his constant help and encouragement, this project would not have been possible.

I would also like to thank the members of my thesis committee, Dr. James L. Vaden and Dr. Jere L. Yates, for their unselfish donation of time and energy to my thesis project. Your guidance and advice were invaluable.

Thank you to Dr. George S. Harris, Dr. James L. Ferguson, and Donna Niemczyk for the countless hours of collecting, measuring and digitizing the orthodontic records used in this research project. I am grateful for their assistance in interpreting the significance of such an impressive collection of research data.

And finally, I am grateful to have been blessed with such admirable parents, Don and Carol Owens, who instilled in me a solid work ethic and sense of responsibility from an early age, and have always been available to offer help and guidance in every way throughout my life. 


\begin{abstract}
Instability of the occlusion is a common problem following orthodontic treatment. The purpose of the present research was to evaluate posttreatment relapse at long-term recall (> 10 years) in cases (A) treated with temporary fixed mandibular retention plus Hawley retainers compared to (B) a similar group retained with just removable retention (standard Hawley type retainers alone). Fixed retainers were removed after having been in place for about 2.5 years. Following this period, use of removable retention was left up to the discretion of the patient in both groups. Data consisted of orthodontic records of 166 American whites, all of whom had received comprehensive orthodontic treatment, collected from multiple private practice orthodontists using conventional Edgewise mechanics. Records were analyzed at pretreatment, end of treatment, and at long-term posttreatment recall examination (mean $=16$ years after treatment). The mandibular Incisor Irregularity index at recall averaged 1.9 $\mathrm{mm}$ in the fixed group and $2.5 \mathrm{~mm}$ in the Hawley-only group. This $1 / 2-\mathrm{mm}$ difference is marginally significant statistically $(P=0.03)$, but seems trivially small clinically. A $1.0 \mathrm{~mm}$ change in Incisor Irregularity during treatment was shown to be associated with $0.13 \mathrm{~mm}$ of relapse long term. Mandibular intercanine width increased (mean $=1.2 \mathrm{~mm}$ ) during treatment, but decreased by almost the same amount by the recall examination. Maxillary and mandibular arch widths became slightly narrower after treatment. Overbite and overjet both increased after treatment. Results of the present study seem more stable than those reported in most long-term studies; however, there is little clinical support for a fixed-retention phase given the modest $(1 / 2-\mathrm{mm})$ difference in Incisor Irregularity.
\end{abstract}




\section{TABLE OF CONTENTS}

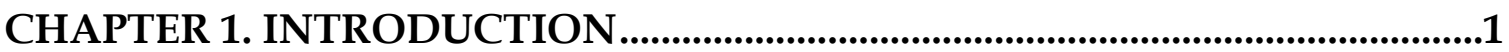

CHAPTER 2. REVIEW OF THE LITERATURE ........................................................

Historical Review of Retention and Relapse............................................................. 3

Etiology of Post-Orthodontic Relapse.................................................................. 5

Arch Dimension and Tooth Extraction ..................................................................

Occlusion and Posttreatment Tooth Position .......................................................10

Periodontal and Gingival Supporting Tissues...................................................14

Mandibular Incisor Dimensions and Approximal Contacts ............................15

Environmental Factors and Neuromusculature................................................16

Continued Physiologic Growth and Development............................................18

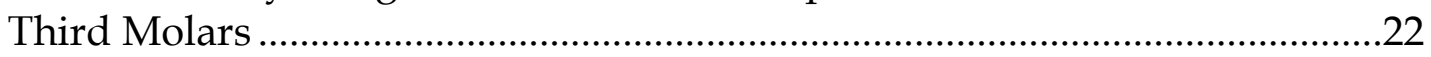

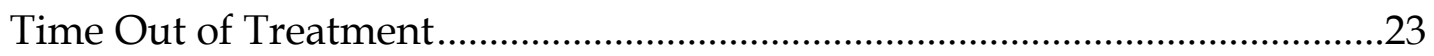

Severity of Pretreatment Malocclusion and Posttreatment Stability...............25

Prevention of Post-Orthodontic Relapse .............................................................. 26

Types of Mechanical Retention .....................................................................26

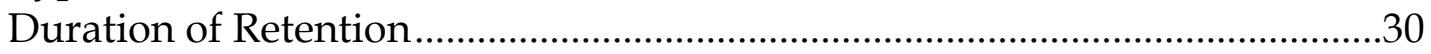

Additional Retention Methods ..........................................................................

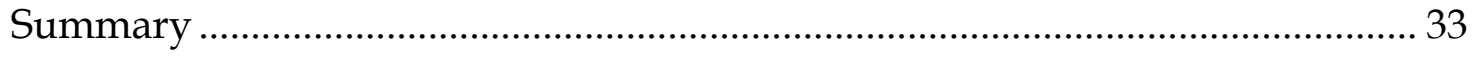

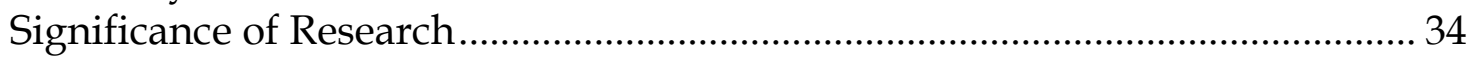

CHAPTER 3. MATERIALS AND METHODS .......................................................36

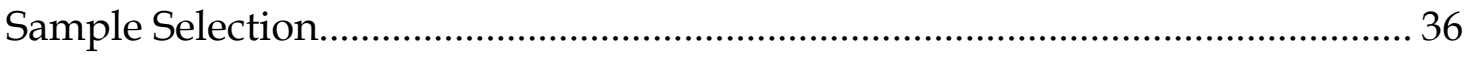

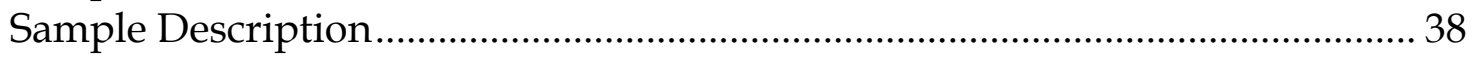

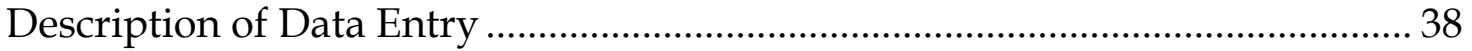

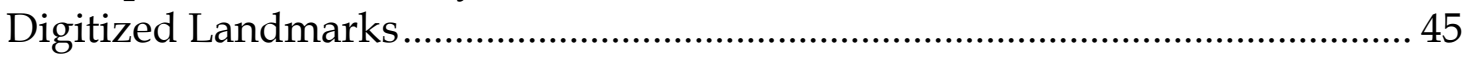

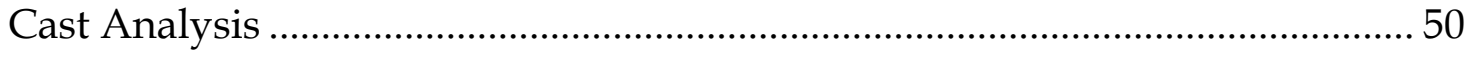

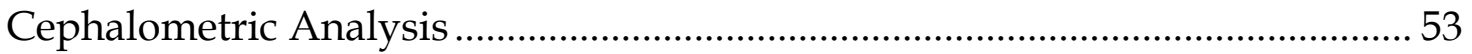

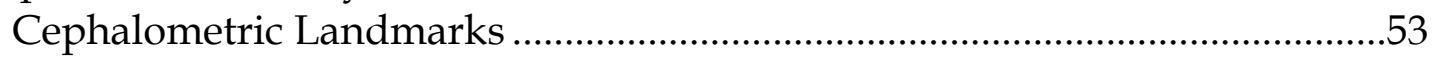

Computed Measurements ..............................................................................56

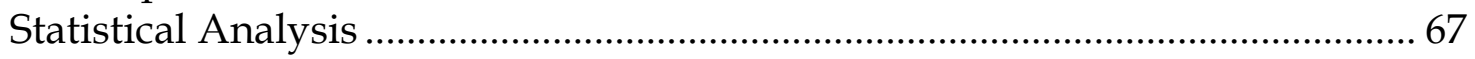

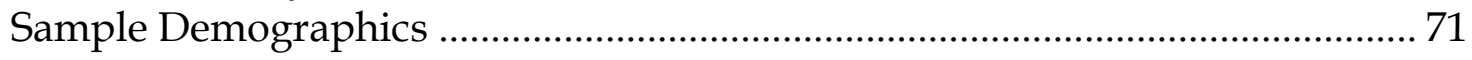

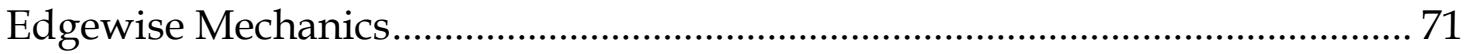

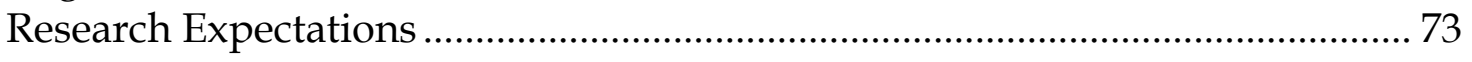

CHAPTER 4. RESULTS ............................................................................................75

Cephalometric and Cast Analysis ........................................................................ 75

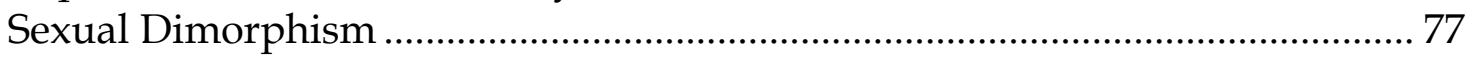

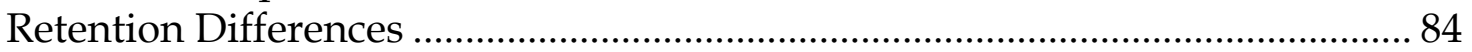

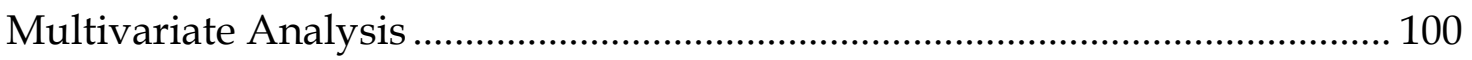

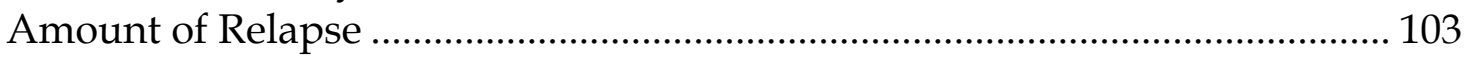

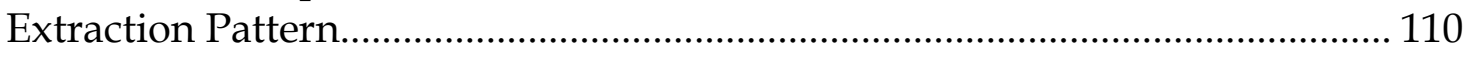


CHAPTER 5. DISCUSSION …............................................................................115

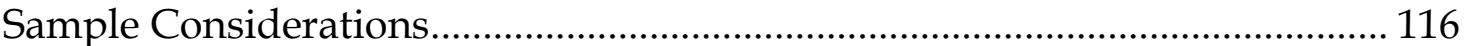

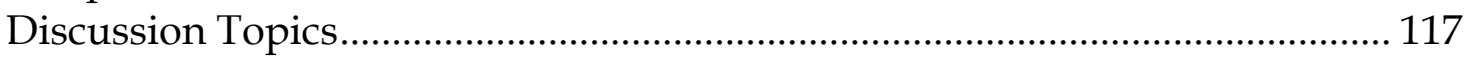

Previous Research and Present Findings.......................................................117

Explanation for Stability ..........................................................................122

Predictors for Fixed Appliances.....................................................................123

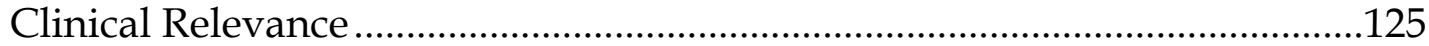

CHAPTER 6. SUMMARY AND CONCLUSIONS.............................................131

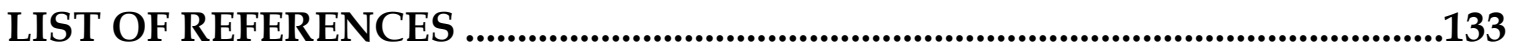

APPENDIX A. DESCRIPTIVE STATISTICS FOR ALL VARIABLES...............151

APPENDIX B. ANCOVA TESTS OF THE COMPARABILITY OF CASES (FIXED VERSUS REMOVABLE RETENTION) WHILE CONTROLLING FOR SEXUAL DIMORPHISM ........................................................................162

APPENDIX C. ANCOVA TESTS EVALUATING WHETHER THE AMOUNT OF CHANGE DEPENDS ON THE TYPE OF RETENTION, WITH PATIENT'S SEX AND TIME-OUT-OF-TREATMENT AS COVARIATES

APPENDIX D. DESCRIPTIVE STATISTICS FOR THOSE VARIABLES EXHIBITING SIGNIFICANCE FOR SEXUAL DIMORPHISM .185

APPENDIX E. EXTRACTION PATTERNS …..................................................191

VITA 


\section{LIST OF TABLES}

Table 3-1 Descriptive statistics for chronologic ages at the three examinations $(\mathrm{n}=166)$

Table 4-1. Results of one-way analyses of variance testing whether the chronologic ages are comparable between the types of retention (fixed or removable) at each of the three examinations and the intervals between them

Table 5-1. Summary of prior studies that investigated the long-term stability of orthodontic treatment for mandibular Incisor Irregularity (II). 


\section{LIST OF FIGURES}

Figure 3-1. All cases $(\mathrm{n}=236)$ available in the Tweed Foundation Study......... 37

Figure 3-2. Illustration of how molar correction was measured........................... 40

Figure 3-3. Illustration showing digitized landmarks......................................... 42

Figure 3-4. Illustration of how the buccal segment angle is constructed. ......... 43

Figure 3-5. Illustration showing midline point and occlusal depth point......... 44

Figure 3-6. Illustration showing anterior segment depth, anterior segment width, and anterior segment ratio ........................................ 46

Figure 3-7. Illustration showing anterior segment perimeter .............................. 47

Figure 3-8. Illustration showing incisor aspect ratio and incisor segment ratio ........................................................................................... 48

Figure 3-9. Illustration showing mandibular segments ........................................ 49

Figure 3-10. Illustration showing occlusal curve. ................................................. 52

Figure 3-11. Illustration of Incisor Irregularity as measured in this study.......... 54

Figure 3-12. Illustration of the incisor overjet and incisor overbite measurements....................................................................................... 55

Figure 3-13. Depiction of the angle ANB (denoted as $\theta$ ) as viewed on a

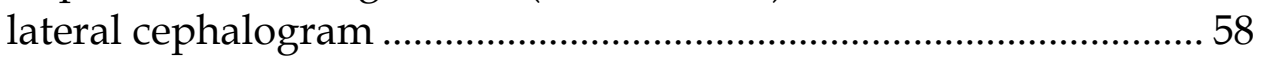

Figure 3-14. Depiction of the horizontal measurement of AOBO from a lateral cephalogram (Jacobson 1975)................................................. 59

Figure 3-15. Illustration of the measurement of FMA (denoted as $\theta$ ) from

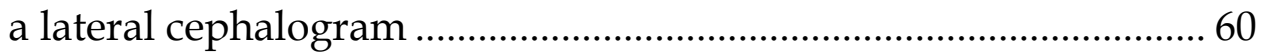

Figure 3-16. Illustration of the measurement of FMIA (denoted as $\theta$ ) from a lateral cephalogram ......................................................................... 61

Figure 3-17. Illustration of the measurement of IMPA (denoted as $\theta$ ) from a lateral cephalogram

Figure 3-18. Illustration of the measurement of the angle SNA (denoted as $\theta$ ) from a lateral cephalogram.

Figure 3-19. Illustration of the angle SNB (denoted as $\theta$ ) on a lateral cephalogram.

Figure 3-20. Illustration of the $\mathrm{Z}$ angle (denoted as $\theta$ ) on a lateral cephalogram. 
Figure 3-21. Illustration of the upper lip measurement on a lateral cephalogram.

Figure 3-22. Illustration of the total chin measurement on a lateral cephalogram.

Figure 3-23. Illustration of the anterior facial height measurement on a lateral cephalogram

Figure 3-24. Illustration of the posterior facial height measurement on a lateral cephalogram

Figure 4-1. Distribution of the 166 subjects according to their years out of treatment.

Figure 4-2. Box plot showing the sex difference in FMIA at the recall examination.

Figure 4-3. Box plot showing the sex difference in FMA at the recall examination.

Figure 4-4. Plot of the average values of $\mathrm{AOBO}$ at the three examinations, by sex.

Figure 4-5. Plot of the average values of FMA at the three examinations, by sex.

Figure 4-6. Box plot of "total chin" at the pretreatment examination based on type of retention. 86

Figure 4-7. Box plot of the distribution in mandibular Incisor Irregularity at the end of treatment according to the type of retention.

Figure 4-8. Box plot of the distribution of the Curve of Spee at the end of treatment in the two retention groups.

Figure 4-9. Box plot of molar relationship at the end of treatment in the two retention groups

Figure 4-10. Box plot of the distribution of Curve of Spee at the recall examination by type of retention......................................................... 91

Figure 4-11. Plot of mean Curve of Spee by examination.

Figure 4-12. Box plot of Incisor Depth at the recall examination by retention group.

Figure 4-13. Box plot of the distributions of mandibular Incisor Irregularity at the recall examination according to type of retention. 
Figure 4-14. Box plot of the distributions of IMPA at the recall

examination.

Figure 4-15. Plot of the mean values for Incisor Irregularity by examination and kind of retention

Figure 4-16. Bar chart of the average changes in Incisor Irregularity by type of retention 98

Figure 4-17. Box plot of Incisor Irregularity at the recall examination, by kind of retention.

Figure 4-18. Bar graph of the means of the four discriminating variables, by group 101

Figure 4-19. Canonical plot of the first two variates that discriminate between the groups treated with a fixed versus just-Hawley retainers.

Figure 4-20. Box plot of the amount of relapse in Anterior Discrepancy (ADisc) dependent on type of retention.

Figure 4-21. Box plot of the amount of relapse in Incisor Segment Ratio (ISR) dependent on type of retention.

Figure 4-22. Box plot of the amount of relapse in Incisor Aspect Ratio (IAR) dependent on type of retention.

Figure 4-23. Box plot of the amount of relapse in Maxillary Intermolar Width (UMW) dependent on type of retention.

Figure 4-24. Box plot of the amount of relapse in Incisor Segment Depth (ISD) dependent on type of retention 108

Figure 4-25. Box plot of the amount of relapse in mandibular Incisor Irregularity (II) dependent on type of retention.

Figure 4-26. Graphic display of Molar Correction (MC) depending on extraction pattern

Figure 4-27. Graphic display of Maxillary Intercanine Width (UCW) depending on extraction pattern

Figure 4-28. Graphic display of Anterior Segment Width (ASW) depending on extraction pattern.

Figure 5-1. Plot of average Incisor Irregularity, by group, at the three examinations.

Figure 5-2. Scatter-plot graph reflecting the association for Incisor Irregularity between the amount of relapse following treatment and the amount of change during treatment. 


\section{CHAPTER 1. INTRODUCTION}

The intent of orthodontic treatment is the "correction and prevention of malocclusion of the teeth" (Tweed 1941, p 5), but the maintenance of the corrections has plagued orthodontists possibly since the inception of the specialty. Hellman (1944) described orthodontic relapse as disturbances occurring in cases that have been successfully treated that lead to a return of the teeth to an undesirable state that may not necessarily resemble the original malocclusion.

Because of the public's perception that orthodontic treatment results should persist indefinitely (Graber 1966) and the fact that stability continues to be a challenge for orthodontists, evidence-based studies are needed to provide information aimed at minimizing relapse. Whether or not individual cases can be viewed as successful or not needs to be evaluated by the stability of treatment results. Rationale and methods of retention following orthodontic treatment continue to be a popular, yet contentious topic within the specialty (Little et al. 1988; Atack et al. 2007; Cerny and Lloyd 2008).

Relapse is the tendency of teeth, which were moved orthodontically to new positions during treatment that may not be well stabilized by the musculature, periodontium and other supporting structures, to return towards their more stable, pretreatment positions (Joondeph and Riedel 1994). Reitan (1959) proposed that teeth tend to return to their pretreatment positions during a period of time following orthodontic correction. In an attempt to establish whether an "equilibrium position" of the postorthodontic dentition is possible, Weinstein et al. (1963) attempted to identify and quantify the effects that various environmental factors might have. Currently however, the various factors proposed to contribute to relapse remain incompletely understood, giving rise to differences in retention protocols among clinicians (Melrose and Millett 1998).

Instability of the dentition following orthodontic treatment exists to some degree in practically every patient (Graber 1994), yet attempts to quantify this degree have proven to be unpredictable (Little et al. 1981). It is widely accepted that the mandibular incisors are the teeth most commonly affected by relapse. In Massler's study based on 2,758 adolescents ranging in ages from 14 to 18 years old, he gave statistical evidence that the mandibular central incisors are the most frequently maloccluded teeth, followed by the mandibular lateral incisors (Massler 1951). Broadbent (1943) and Fastlicht (1970) also noted the higher incidence of crowding in the lower incisor region as compared to other areas of the dentition in both treated and untreated people. 
Joondeph and Riedel (1994, p 908) defined orthodontic retention as "...the holding of teeth in ideal esthetic and functional positions." The goal of retention is to counteract forces that might affect the teeth as a result of their new positions in the oral environment until such time as the teeth and supporting tissues have become reoriented to the new position. The standard Hawley retainer and the fixed mandibular canine-to-canine retainer have remained popular methods to help achieve these goals and are in routine use by many orthodontists today. Some orthodontists suggest that fixed retention is the only way of ensuring a stable result (Angle 1907; Little et al. 1988), while others contend that removable appliances are required to enable the supporting tissues to adapt to the functional demands placed on them (Hawley 1919; Markus 1938; Waldron 1942). Studies have documented the importance of retention as a helpful adjunct of orthodontic treatment to retard relapse, but few studies offer long-term information focusing on the benefits of temporary fixed retention of the mandibular anterior teeth. Due to the scarcity of research is this area and because ambiguities regarding posttreatment retention and relapse continue to exist in both the literature and among practitioners, the present study aims to review the retention problem and address the effectiveness of placing mandibular canine-to-canine fixed retention for a period of two to three years following comprehensive orthodontic treatment.

The purpose of the present research project is to evaluate Incisor Irregularity and relapse in a sample of people at long-term recall who were treated with temporary fixed mandibular retention following comprehensive orthodontic treatment, and compare these results to a similar group of patients at long-term recall who were given removable retention, i.e., standard Hawley type retainers at posttreatment. By evaluating pretreatment, posttreatment and longterm recall records of patients all treated under a single treatment philosophy, an effort is made to clarify the effects of using prolonged mandibular fixed retention on long-term stability. 


\section{CHAPTER 2. REVIEW OF THE LITERATURE}

\section{Historical Review of Retention and Relapse}

No other issue in orthodontics, with the possible exception of extractions, seems to receive as much attention as retention. Retention, as it pertains to orthodontics, has been defined by Moyers (1973, p 442) as "the holding of teeth following orthodontic treatment in the treated position for the period of time necessary for the maintenance of the result" (Blake and Bibby 1998, p 299). Over time, there have been different schools of thought regarding the prevention of relapse (Graber 1994). Henry Kaplan (1988) devoted a review article to the issue of retention. He noted that the problems associated with retention continue today and are just as debated and relevant now as they ever have been despite the considerable amount of attention the subject has received. Much of the early historical references found in this section were taken from Kaplan's review of retention and also from George Huckaba's analysis of relapse (1952).

Although early clinicians recognized changes in the dentition following treatment, Evens in 1854 seems to be the first to mention the need for some type of retention. Emerson Angell in 1860 likewise recognized a need for retention following the expansion of the maxillary median suture (Weinberger 1926). Alfred Coleman (1865) was the first to describe the effects of muscular pressure on the dentition, in essence relapse, and one year later, C. A. Marvin described the physiologic reasons for retention (Weinberger 1926).

Around the beginning of the twentieth century, the issue of retention had become widely recognized, and many prominent clinicians and authors such as Guilford (1898), Angle (1900), Lischer (1912), and Dewey (1914) included chapters dealing with retention in their texts. Kingsley (1908) recognized that there were challenges associated with retention, and admitted that regarding the retention issue, "I am agnostic, I don't know, in each and every individual case I do not know" (Kaplan 1988, p 327). It was the opinion of Edward H. Angle $(1900,1907)$ that achieving a normal occlusion as a result of orthodontic treatment was the most crucial component necessary in achieving long-term stability, even at the expense of an expanded dental arch. In contrast, McCauley (1944) promoted maintaining inter-canine and inter-molar widths of the original malocclusion. In accordance with McCauley, Tweed $(1944,1945)$ also recognized the lack of stability in cases where arch expansion had occurred, and Tweed went on to advocate the extraction of teeth in order to prevent this. Tweed, along with Nance (1947), also proposed that the most stable position for mandibular incisors was upright over their supporting basal bone. 
Strang (1952, p 794) claimed that "the primary causes of malocclusion lie hidden in the mysterious processes of growth and development, which are beyond our power to control," and Strang recommended overcorrection as a means of reducing the incidence of post-treatment relapse. Reitan $(1959,1967)$ noted the potential effects that the periodontium and supporting structures could have on tooth position. Jackson (1904) and Edwards $(1968,1970,1988)$ each described procedures aimed at reducing this influence by surgically separating the attached gingiva from the teeth for a period of time. Boese (1980) also advocated a surgical reduction of interproximal tooth structure to reduce the instability of teeth.

Edward H. Angle (1907) wrote that the problems of retention are often taken too lightly and are greater than the problems encountered during active treatment. Perhaps during a time of extreme frustration, Hawley (1919) proclaimed that he would "give half of his fee to anyone who would be responsible for the retention of his results when the active appliance was removed" (Kaplan 1988, p 328). Milo Hellman (1936) stated that, "We are almost in complete ignorance of the factors which pertain to retention for the individual patient" (Kaplan 1988, p 325), and George Hahn (1944) referred to retention as "The Stepchild of Orthodontia."

Boley and coworkers (2003, p 277) showed optimism that long-term stability is not hopeless and that, "satisfactory long-term results can be achieved for most patients for whom evidence-based treatment objectives - including minimal alteration of the mandibular arch form and the retraction and uprighting or maintenance of mandibular incisors in their original position have been met." Based on his review of the literature on post retention stability of mandibular incisors, Boley (2007) also supported the contention of Little and coworkers (1990) who looked at retention of early treatment cases involving arch length increases, and concluded that decisions made during active treatment, such as lateral arch expansion and advancement of the mandibular incisors, make a difference in long-term stability.

Levin (1972) stated that, "It is the obligation of the clinician to plan the retentive phase of orthodontic therapy for all adult patients before active treatment is begun (Kaplan 1988, p 326)." Graber (1994, p 909) noted that, "retention depends on what is accomplished during treatment." This requires careful consideration of the factors that have the potential to affect posttreatment outcomes and stability. 


\section{Etiology of Post-Orthodontic Relapse}

Melrose (1998, p 507) stated that, "Stability can only be achieved if the forces derived from the periodontal and gingival tissues, the orofacial soft tissues, the occlusion and posttreatment facial growth and development are in equilibrium." Since it appears that numerous factors affecting stability are under the control of the orthodontist and that many are of the opinion that there is no phase of orthodontic treatment that is more important than the functional retention of the finished case (Waldron 1942), the following sections serve to review the etiologies surrounding posttreatment relapse as well as the methods commonly used to minimize its occurrence (Huckaba 1952).

In an attempt to summarize the accepted opinions regarding retention that have influenced the specialty, Riedel (1960) derived several "rules" or "theorems" based on his review of the literature from such noted clinicians and researchers as Kingsley (1880), Angle $(1900,1907)$, Hawley (1919), McCauley (1944), Tweed $(1944,1945)$, and Reitan $(1959,1967)$, among others. Riedel (1960) recognized that posttreatment stability should be a prime objective in orthodontic treatment, and he intended these rules to serve as guidelines to be considered throughout treatment to aid orthodontists in the diagnosis, treatment planning and active phase of orthodontic treatment. By doing this, clinicians could expect that each case would stand the best chance of achieving a stable, long-term posttreatment result. These perceptions have since been periodically updated and now serve as the backbone of information presented by Joondeph (2005) in the current edition of the popular Graber textbook (Graber et al. 2005). Because relapse is a complex problem that is believed to be multifactorial in nature, Riedel's "theorems" as well as other factors and techniques currently thought to influence posttreatment relapse are considered in the following sections.

\section{Arch Dimension and Tooth Extraction}

The size, form, and width of the alveolar dental arches are important factors that should be considered not only in the diagnosis and treatment planning of orthodontic cases, but also in the maintenance of treatment results. Lee (1999, p 305) found in his study of the literature that, with regard to arch width and form, "A variety of opinions exist on the potential for change in arch dimensions." Historically, practitioners have been broadly classified into two groups based on their opinions regarding this issue: those who contend that arch dimensions may be altered in order to meet specific treatment goals (expansionists), and those who contend that arch dimensions of the pretreatment 
malocclusion must be maintained if long-term stability is to be achieved (nonexpansionists).

Angle (1907, p 63) contended that each individual had the potential for normal growth and development as evidenced by his statement, "The best balance, the best harmony, the best proportions of the mouth in its relation to the other features, requires that there shall be a full complement of teeth and that each tooth shall be made to occupy its normal position-normal growth." Because Angle contended that long-term stability hinged on a full complement of teeth maintained in the proper occlusion, expansion was naturally incorporated into many of his treated cases. As a result, cases were often treated with acceptable dental and occlusal relationships at the expense of altered arch dimensions and facial esthetics (Tweed 1944, 1966). Because of Angle's prominence and the influence he had in the field of orthodontics at the time, his opinions became widely accepted within the orthodontic community.

Calvin Case $(1911,1921,1964)$ was one of the earliest clinicians to recognize the relationship between arch dimension and stability. Case alleged that the relapse seen is some of his patients, especially in the mandibular incisors, was the result of teeth being positioned outside the natural confines of their supporting bone. In an effort to correct this, Case became the first orthodontist to extract teeth in an effort to maintain arch dimension. Shortly thereafter, Axel Lundström (1925) also recognized the importance of maintaining an adequate apical base in relationship to the teeth, and accepted tooth extraction as a means of achieving this result.

McCauley (1944) stressed the importance of maintaining the intercanine width throughout treatment. It was his opinion that the Class I canine relationship (i.e. the mandibular canine travels mesial to the maxillary canine in lateral excursions) is the primary determinant of functional occlusion and arch form. McCauley (1944) also noted the importance of arch width when he recommended that because molar width and cuspid width are of such an uncompromising nature, one might establish them as fixed quantities and build the arches around them.

Charles Tweed was perhaps the most notable early proponent of tooth extraction. Tweed $(1944,1945,1952,1966)$ wrote extensively on tooth extraction and the significance of the relationship of the teeth to their apical bases. Tweed was a student of Angle, who, like others at the time, became dissatisfied with not only the instability of his results, but also the facial profiles of his treated patients. Tweed felt that there was a lack of beauty and harmony associated with the face, when the teeth, particularly the lower incisors, where not placed in the proper relationship to their apical bases. So he decided to retreat 100 of his cases 
by extracting bicuspids in an effort to circumvent the arch expansion that was created as a result of his previous non-extraction treatment. Tooth extraction allowed him to: create the space necessary to relieve dental crowding; position the mandibular incisors "upright over basal bone," into what he considered their most stable position; and prevent the expansion of the dental arch.

Strang (1949) also opposed expansion of the dental arches and claimed that expansion of the dental arches would not remain stable. Strang (1949, p 17) stated, "There is no question in my mind that denture expansion as a treatment procedure in the correction of malocclusion should be discarded and every effort should be directed toward preserving the muscular balance that is the most important factor in establishing tooth position." Strang (1949, p 16) asserted that in cases where it was impossible to align the mandibular incisor teeth in an upright position over basal bone without the buccal movement of the cuspids and posterior teeth, the reduction of tooth material by the "extraction of dental units is definitely indicated." Strang (1952) went on to assert that pretreatment intercanine width dictated the limit of acceptable denture expansion that would allow for stability of the finished result.

In contrast, Douglas Walter (1953) proposed that a crowded or otherwise deformed pretreatment arch did not necessarily represent its true size or form and that maintaining arch dimension was no guarantee in achieving a stable posttreatment result. Walter based his opinions on 102 non-extraction cases ranging in age from 6 to 36 years of age. Pretreatment dental casts were taken of all 102 cases. These were compared to 34 dental casts that were available immediately after active treatment and to 90 dental casts available from intervals ranging from one to 13 years (average of 2.5 years) after all retention had been removed. Bilateral measurements were made between every pair of teeth of both arches and arch length was also recorded by measuring the distance along the arch between the mesial of each first molar. Walter found that permanent expansion of arch widths occurred in $88 \%$ of the patients, and in some of these cases, expansion continued past the active treatment phase. Walter found that $12 \%$ of expanded cases did return to their more narrow pretreatment arch form. Similar findings were noted for arch length as well. Walter claims that all cases maintained the occlusal result gained in treatment and that all but $10 \%$ of the cases could be viewed as highly successful cases. The conclusions drawn from this study was that a wide variety of results can be found when arch dimension is altered during treatment and that preservation of arch dimension is no guarantee of future stability.

In his review of the literature in 1960, Riedel concluded that arch form, particularly in the mandibular arch, should not be permanently altered by orthodontic treatment. Instead, Riedel (1960, p 181) asserted, "Treatment should 
be aimed at maintaining, in most instances, the arch form presented by the original malocclusion."

Bishara et al. (1973) examined a sample of 30 treated cases from the University of Iowa, Department of Orthodontics. All patients were treated with the edgewise appliance and required first premolar extractions. The mean postretention time was 14.8 months, with a range from 6 months to 48 months. Pretreatment, posttreatment, and postretention dental casts were used to measure maxillary and mandibular intercanine width. The results indicated that in cases where mandibular intercanine expansion occurred, the mean percentage relapse was $71 \%$. However, where intercanine expansion occurred in the maxillary arch, the mean percentage relapse was only $3 \%$.

Herberger (1981) concluded that some patients can be treated with canine expansion and that this expansion can be maintained. Herberger looked at the stability of mandibular intercanine width at various retention periods. He based his study on a sample of 56 of his own patients whom he treated nonextraction, and cases were divided according to the length of retention, namely 4, 5, or 6 years. The intercanine widths of all patients were measured from casts at pretreatment, posttreatment, and at postretention. Of the 56 cases, 52 showed mandibular intercanine expansion, 2 remained unchanged, and 2 were constricted $1 \mathrm{~mm}$ during treatment. Most cases (38 patients) remained expanded to some degree, while 18 lost an average of $0.8 \mathrm{~mm}$ from their initial intercuspid width. Herberger found no case in which the mandibular intercuspid width increased after retention.

Little, Wallen, and Riedel (1981) assessed 65 patients who had been treated with first premolar extractions and traditional edgewise mechanics at a minimum of 10 years posttreatment. Retention methods were varied. Arch width decreased an average of $2 \mathrm{~mm}$ after retention, regardless of treatment expansion or constriction. The researchers concluded (p 357) that, "Arch width change during treatment was a poor predictor of long-term crowding, the degree of expansion or constriction having little association with postretention alignment." Arch length was also found to decrease during treatment due to extraction, but also found to decrease during the postretention period as well.

Researchers continue to explore the ramifications associated with the expansion of the dental arches during orthodontic treatment. One possible explanation for the continued interest in canine expansion is the fact that canine expansion is responsible for creating usable space in the transverse dimension to correct crowding (Gianelly 2006). According to Ricketts et al. (1982), $1 \mathrm{~mm}$ of intercanine width expansion results in a $1 \mathrm{~mm}$ increase in arch perimeter, while a 
$1 \mathrm{~mm}$ expansion of the intermolar width provides only a $0.25 \mathrm{~mm}$ increase in arch perimeter.

De La Cruz et al. (1995) evaluated records of 45 Class I patients and 42 Class II, division 1 patients to evaluate the long-term stability of orthodontically induced change in arch form. Dental casts were analyzed at pretreatment, posttreatment and a minimum of 10 years posttreatment. These researchers concluded that arch form tends to return toward its pretreatment shape and that the patient's pretreatment arch form appears to be the best guide to future stability. Although the researchers found a positive correlation between degree of treatment arch change and degree of posttreatment relapse, they claimed that minimizing treatment arch form change was no guarantee of postretention stability.

Vaden, Harris and Gardner (1997) quantified changes in tooth relationships in a sample of 36 extraction patients at 6 years and again at 15 years posttreatment. Although the maxillary intercanine width was expanded more than the mandibular intercanine width during treatment, both arches were expanded slightly, but to a statistically significant extent. An interesting observation noted during this study was that with any study of this type, "it is difficult to determine whether intercanine 'expansion' occurred by a transverse movement of the teeth or retraction of the canines in to the premolar extraction spaces, a broader part of the arch" ( $p$ 545). Posttreatment results indicated that a significant amount of the maxillary expansion was maintained, while half of the expansion that occurred in the mandible during treatment was lost. Maxillary and mandibular arch length was reduced an average of $5.7 \mathrm{~mm}$ during treatment as a result of tooth extraction, but it also continued to decrease an average of 1 $\mathrm{mm}$ throughout the two recall periods even though no spacing was left following active treatment. This continued reduction in arch length was attributed to the mesial migration of teeth in the buccal segment. Both arches become shorter and narrower with age. These results reiterate the conclusions of previous researchers who found that posttreatment arch dimension changes and dental crowding can be minimized and kept similar to changes seen in untreated samples by maintaining pretreatment arch dimension and keeping alterations in the intermolar and intercuspid distances to a minimum during treatment (Steadman 1961; Lundström 1968; Glenn et al. 1987; Bishara et al. 1989).

Burke et al. (1998) applied a meta-analysis technique of literature review to a total of 26 studies to assess the longitudinal stability of posttreatment mandibular intercanine width. Glass $(1976, \mathrm{p} 3)$ defines meta-analysis as "the statistical analysis of a large collection of results from individual studies for the purpose of integrating findings." Weighted averages and standard deviations for the means of 1,233 patients were analyzed for changes in intercanine width at 
three time intervals: during treatment, immediately following treatment, and after removal of all retention. Regardless of pretreatment molar classification or whether treatment involved extractions, mandibular intercanine width was found to expand during treatment by 0.8 to $2.0 \mathrm{~mm}$. However, at posttreatment, the mandibular intercanine width constricted by 1.2 to $1.9 \mathrm{~mm}$ and showed a net change in posttreatment on the order of from $0.5 \mathrm{~mm}$ expansion to $0.6 \mathrm{~mm}$ constriction. The results of this meta-analysis support the concept of maintenance of the original intercanine width during orthodontic treatment.

In conclusion, the literature suggests that maintaining the patient's existing arch dimensions, with the possible exceptions of patients who exhibit malformed or mutilated arches, results in the most stable posttreatment results. There is also a direct correlation with the ability to maintain arch dimension and tooth extraction. Historically, orthodontists were left with only two treatment alternatives when faced with a crowded dental arch: either expansion of the arch or tooth extraction. Lindauer (1997) emphasized that when considering extraction of teeth, other contributing factors should be evaluated in addition to intra-arch crowding. These include "the predicted effect of treatment on lip protrusion, the amount of overbite present, the patient's periodontal condition, and whether or not there are missing or compromised teeth" ( $\mathrm{p} 31)$. For each millimeter of incisor advancement or flaring, the arch perimeter is increased by 2 mm (Ricketts et al. 1982; Lindauer 1997). In addition, arch width increases are possible but the space yielded is smaller for any given amount of expansion (Ricketts et al. 1982; Germane et al. 1991; Lindauer 1997).

The literature review suggests that arch dimension changes resulting from treatment modalities such as minor arch expansion and minimal incisor flaring can be successfully utilized in patients exhibiting mild crowding without substantially diminishing posttreatment stability. However, in cases with moderate to severe space requirements, such as severe dental crowding or anterior protrusion cases, where excessive arch expansion or incisor flaring would be necessary to relieve the tooth-arch discrepancies, tooth extraction continues the be the most appropriate treatment modality to best allow for predictable arch dimension maintenance and thus prolonged posttreatment stability.

\section{Occlusion and Posttreatment Tooth Position}

The establishment of a functional and stable posttreatment occlusion should be a primary objective of orthodontic treatment. Kingsley (1880, p 63) stated, "The occlusion of the teeth is the most potent factor in determining the 
stability in a new position." Angle (1907) asserted that orthodontic corrections would remain stable if the teeth were placed in proper occlusion.

Dental "occlusion" refers to how well the teeth are arranged individually and one-to-another within and between the dental arches (Harris and Corruccini 2008). "Proper" occlusion occurs when the mesiobuccal cusp of the maxillary first molar is positioned parasagitally in the buccal groove of the mandibular first molar (Angle 1899; Harris and Corruccini 2008). According to Angle, the achievement of this Class I molar relationship was a prerequisite in obtaining the proper interdigitation of the maxillary and mandibular cusp-fossa relationship. According to Huckaba (1952), Dewey and Anderson (1942) suggested that, in addition to the inclined plane relationship of opposing teeth, a proper cuspal guidance will serve as a natural aid to retention. According to Kaplan (1988, p 328), McCauley (1944) also stressed his opinion that the achieving of a proper canine relationship is the primary determinant of a functional occlusion and referred to the canine rise as a "protective mechanism for maintaining arch stability." Occlusion is a dynamic balance of forces in which the maintenance of form is subject to functional requirements (Huckaba 1952).

Tweed (1944) added further to the requirements of occlusion based upon the treatment results of some of his early cases. Based on the many patients he recalled years after treatment, Tweed concluded that there were several factors that must be achieved during treatment for a case to be deemed successful. These included a normal cusp and inclined plane relationship between opposing teeth; ideal axial orientations of all teeth; ideal relationship of the jaws to each other and to the skull; and, there must exist an ideal function of all masticatory elements involved. Tweed (1944) asserted that achievement of these objectives was dependent on the positioning of the mandibular incisor teeth over basal bone. Tweed (1945) claimed that the mandibular incisors should be treated to a 90 degree angle in relationship with the mandibular plane in order for incisor stability to occur.

Strang (1949) suggested that the mandibular cuspid and mandibular first molar teeth are the key in determining the alignment pattern for the remaining teeth so that the dentition will be in harmonious balance with the muscular forces which constantly act upon it. Strang felt that if muscular balance was maintained in this manner, it should be possible to eliminate mechanical retention at the end of active treatment and-have a result that would remain stable.

According to Lundström (1925), the presence of an adequate apical base is necessary for the correct positioning of the teeth in proper occlusion. He claimed that orthodontic experiments show that a normal occlusion attained by 
mechanical treatment does not necessarily lead to the development of the apical base in harmony with the newly positioned teeth. This failure to attain bony support would lead to future instability. Lundström concluded that the apical base does not change to fit the occlusion, but that apical base dimensions dictated the establishment of a normal occlusion.

Andrews (1972) observed the casts of 120 nonorthodontic patients in an effort to describe common characteristics that lead to the establishment of a normal occlusion. The patients were selected form the general public because they possessed a dentition that was well aligned and pleasing in appearance; a bite that looked generally correct, and no potential benefit from orthodontic treatment according to the author. From these nontreated patients, 6 common features or "keys" were found to be present.

Andrews' six keys to normal occlusion are:

1. Molar relationship. The distal surface of the distobuccal cusp of the maxillary firs molar contacts with the mesial surface of the mesiobuccal cusp of the mandibular second molar.

2. Crown angulation (tip). The gingival portion of the long axes of all crowns should be more distal than the incisal portion.

3. Crown inclination. The maxillary incisors should exhibit slight labial crown torque and all posterior teeth from the canine through the second molars should exhibit lingual crown inclination.

4. Rotations. All teeth should be free of undesirable rotations.

5. Tight contacts. The contact points between adjacent teeth should be tight and exhibit no spaces.

6. Occlusal plane. Occlusal planes should be treated such that they are flat or exhibit only a slight Curve of Spee.

Andrews concluded that retention was not necessary in patients who possessed all six keys of occlusion, provided the third molars had been removed and growth had ceased.

Harris and Behrents (1988) assessed the relative stability of the sagittal molar relationship in 61 orthodontically untreated persons with full dentitions who were recalled from the Bolton longitudinal study conducted in Cleveland, Ohio. The investigators found that $100 \%$ of the patients that originally presented 
with Class I molar were found to maintain the Class I relationship more that 30 years later. In contrast, the Class II relationships were found to become more Class II (exhibit greater distoclusion), and the Class III cases shifted significantly to greater mesioclusion. Harris and Behrents concluded that a Class I molar relationship was the most stable, while Class II and Class III relationships of molars rarely self-correct, but likely will progressively worsen over time.

Riedel (1976) questioned the importance of treating all patients to mean cephalometric parameters as advocated by Tweed (1945) and suggested that the pretreatment incisor position is likely the most stable position for that particular anterior segment. Other studies (Shields et al. 1985; Houston and Edler 1990) have also supported this. Research by Weinstein et al. (1963) and Mills (1966) suggests that the mandibular incisors lie in a narrow zone of stability in equilibrium between opposing muscular pressures, and that the pretreatment labiolingual position of the incisors should be accepted and not altered by orthodontic treatment (Blake and Bibby 1998). The importance of occlusion also comes into question when it is realized that a normal posttreatment occlusion may only be temporary, since the stability of teeth are often influenced by many factors, such as posttreatment growth that cannot be accounted for. It has also been realized that a settling of the occlusion usually occurs to some extent following treatment. Although it is usually anticipated that this posttreatment settling results in more occlusal contacts and thus a more stable occlusion (Razdolsky et al. 1989; Sullivan et al. 1991), this certainly cannot occur in all cases and it has been shown that the degree and amount of posttreatment settling can vary (Morton and Pancherz 2009).

Sauget et al. (1997) compared the occlusal changes occurring between patients who wore a Hawley retainer and patients who wore a clear overlay retainer full-time only for the first three days (except during meals), followed by nighttime wear only. There was a statistically significant increase in the amount of settling that occurred in the Hawley group than in the clear overlay group after three months of retention. There was a mean difference of 6.5 occlusal contacts between opposing teeth in the two groups. However, the authors did not elaborate as to whether or not these additional contacts were in the correct locations.

According to Alexander (2008), ideal occlusal contacts play an important role in maintaining occlusal stability. Reitan (1959) found that less relapse of mesiodistal movement occurs in the absence of occlusal stress and parafunction, suggesting that contact points must be in the correct locations. Dawson (2007, p 12) claimed that, "the use of long-term retainers to maintain post-orthodontic tooth alignment could be dramatically reduced if occlusal principles were better understood." Without stable stops on all teeth when the condyles are in centric 
relation, the masticatory system will not be in equilibrium, and signs of occlusal instability will occur over time (Dawson 2007). These signs could include TMJ problems, occlusal wear facets, unhealthy supporting structures, and unstable tooth positions. A proper, functional occlusion consisting of ideal contacts should be attempted during treatment such that the favorable vertical forces produced by proper interdigitation of the opposing dentitions can serve as a natural means of retention (Dinçer et al. 2003). Despite conflicting opinions, Rosenstein and Jacobson (1971) suggest that many clinicians believe that there is much truth in the clinician's lament: A good sock-in and parallel roots, and the case should hold!

\section{Periodontal and Gingival Supporting Tissues}

Residual forces from the surrounding musculature and alveolar fibrous system can continue to influence the teeth toward their pretreatment position (Reitan 1959, 1967; Moss 1980; King and Keeling 1995; Tanne et al. 1995). This is because the fibers of the periodontal membrane extend into the gingiva, into the adjacent gum tissue, and into the crest of the alveolar process and serve to bind the whole group into a compact mass around the neck of a tooth (Black 1936).

The premise of posttreatment orthodontic retention is based on histological research that shows that it takes time for the surrounding periodontal tissues to reorient following tooth movement (Reitan 1959, 1967; Rygh et al. 1986). Reorganization of the periodontal ligament is estimated to occur over a period of 3 to 4 months following orthodontic treatment (Reitan 1959, 1967, 1969; Melrose and Millett 1998), whereas the gingival collagen-fiber network typically takes 4 to 6 months to be remodeled, and the elastic supracrestal fibers have been shown to remain deviated for more than 232 days (Reitan 1967; Melrose and Millett 1998). Conclusions regarding mandibular incisor crowding based on the research by Southard et al. (1992) suggest that the periodontium exerts a continuous force by means of transseptal fibers on the teeth, which acts to maintain the contacts of approximating teeth in a state of compression. This suggestion may help to explain the long-term crowding of the mandibular anterior teeth.

Orthodontic correction involving rotated teeth tends to be one of the more difficult tooth movements to maintain. Reitan (1959) suggests that this type of tooth correction results in stretched supra-alveolar collagen fibers that exert tensions which pull the teeth back toward their pretreatment positions. According to Reitan $(1959,1967)$ and Edwards $(1968)$, these fibers can remain stretched and continue to exert pressure for extremely long periods of time. For this reason, various authors suggest surgical means following orthodontic 
correction in order to sever these tissue attachments that are thought to contribute to the relapse (Thompson 1959; Edwards 1970, 1988; Boese 1980).

Redlich et al. (1996) also examined the posttreatment relapse tendencies of rotated teeth, but contrary to Reitan and Edwards, came to different conclusions regarding stretched collagen fibers. Redlich et al. had the benefit of scanning and transmission electron microscopes to analyze control and experimentally rotated tissue samples. Results indicated that the rotational forces produced gingival tissue changes that were inconsistent with stretching. Following resection of these tissue fibers, many of these periodontal fibers assumed positions similar to their pretreatment positions and the positions of the control groups.

\section{Mandibular Incisor Dimensions and Approximal Contacts}

Theories have been put forth concerning the association between mandibular incisor dimensions and crowding. Begg (1971) proposed that the interproximal surfaces of teeth, particularly the incisors, become worn over time due to function. As this attrition takes place, the interproximal contacts between adjacent teeth evolve from a smaller, point type contact to a broader and flatter contact that he proposes to be a natural aid to stability. This interproximal attrition also reduces the mesiodistal width of the teeth, which allows for the mesial migration of teeth in response to the constant mesial forces present on them. In essence, Begg attributes dental stability to changes in shape and size of the approximal contacts of mandibular incisors.

Moore (1956) compared the mandibular dental arch with the staves of a barrel; as long as all of the staves in a barrel are in contact with one another, the barrel remains intact. He noted that the lingual and distal functional forces acting on the lower incisors must be offset or resisted by what he termed the "contained arch principle" (p 53) and possibly pressure exerted by the tongue. This contained arch principle depends on each of the lower incisors having solid, broad contacts with each other, thus balancing the opposing forces without slipping. Like Begg, Moore suggested that flat, worn contacts were better able to balance opposing forces and minimize slippage than were smaller, point contacts.

According to Huckaba (1952), Dewey and Anderson (1942) also suggest that approximal contacts are important, natural forces of retention. They made the comparison of the dental arch to a masonry arch in which each unit exerts a passive force to maintain itself and its fellows in alignment. In just the same manner, if a unit of the dental arch is removed or distorted by a faulty restoration, a lack of stability will occur. All the active muscle forces that are 
exerted on the denture from the buccal side are resisted by approximal contacts of the individual teeth.

The importance of achieving an optimal functional occlusion as it relates to stability has already been discussed, but it is worth mentioning how the size of the mandibular teeth as they relate to maxillary teeth influence occlusion and, thus, stability. Because discrepancies in tooth size and shape have been shown to be factors associated with malocclusion (Ballard 1944), Bolton (1958) analyzed 55 cases with excellent occlusions in an effort to determine whether or not mathematical ratios could be set up between total lengths of dental arches, as well as between segments of dental arches. The well known Bolton analysis or ratio is the result of this research. Bolton determined that for proper interdigitation of opposing dental arches, the mandibular teeth should be approximately $77.2 \%$ of the size of the maxillary teeth.

Peck and Peck (1972) sought to determine if there are distinctive dimensional characteristics related to naturally occurring well aligned mandibular incisors. The study consisted of 2 samples of patients: one sample contained 45 untreated patients with perfect mandibular incisor alignment; and, the second sample consisted of 70 patients who exhibited anterior crowding. Mesiodistal and faciolingual crown diameters of the mandibular incisors were recorded for each patient. The lower incisors in the perfect alignment group were significantly smaller mesiodistally, but significantly larger faciolingually than the incisors in the crowded group. It was concluded that crown shape was one of several possible determining factors correlated with lower incisor crowding.

Results of the Peck and Peck (1972) research have been disputed in the literature. Gilmore and Little (1984) studied 134 treated and 30 control cases from the University of Washington at a minimum of 10 years postretention. Statistical results indicated only a weak association between incisor width or the faciolingual/mesiodistal ratio and long-term lower Incisor Irregularity. It was found that only $6 \%$ of the variation in crowding could be explained by this ratio. Other studies (Smith et al. 1982; Puneky et al. 1984) confirm these findings, suggesting that crown form plays only a minor part in the occurrence of mandibular incisor crowding.

\section{Environmental Factors and Neuromusculature}

It is well documented that adverse muscle habits play a role in defining

not only tooth position, but also arch shape. Strang (1949) noted that every malocclusion represents a denture under the influence of and stabilized by 
muscular balance. Successful treatment, as evidenced by permanent stability, must preserve this muscular balance rather than attempt to alter or upset it.

Moss (1969) described a functional matrix theory that suggests that the form, position, and maintenance of the denture are secondary responses to the primary demands of the related muscles acting on it. Moss contended that change or activity in the surrounding muscles would impact the positions and stability of the teeth and alveolar bone. This accounts for the many changes that are commonly seen following active orthodontic treatment. The muscles and soft tissues become the natural retentive forces of the newly acquired position of the dentition, so aberrant muscle actions present during swallowing, speaking, chewing, or breathing are likely to manifest themselves on the dentition and skeletal bases.

Huckaba (1952) referred to the soft tissue environment when he suggested that the natural forces of retention must be considered when treatment is planned. If this is not done, the forces of retention may become the forces of relapse. Moss's model supports the use of various functional appliances such as the Fränkel appliance (Fränkel and Fränkel 1989) and the lip bumper. Such appliances act on the surrounding tissues in an effort to change muscle and tissue functions to allow for a balance between soft tissues and the newly acquired position of the dentition.

In an effort to study the effects that muscles can have on the form of bones, Moyers (1949) used electromyography to study the electrical phenomena occurring within muscles as a means of analyzing the nature of the muscle contraction, particularly the role of the temporomandibular musculature in the etiology of Angle Class II malocclusions. By measuring the electrical impulses, it became possible to achieve a more accurate understanding of muscular forces present in the oral environment.

At the University of Washington, Proffit et al. (1964) evaluated 25 young adult dental students ranging in age from 22 to 32 years old to record tongue and lip pressures against the dentition in a "normal" sample of people. Three stain gauge pressure transducers were placed intraorally for each subject so as to allow simultaneous recording: (1) labial to the maxillary central incisors, (2) lingual to the maxillary central incisors when the teeth were in occlusion, and (3) lingual to the maxillary first molar. Intraoral pressures were found to vary greatly among individuals, but on average were less than had been previously recorded in the literature (Gould and Picton 1962; Weinstein et al. 1963). Increasingly higher pressures, as a result of greater tongue and buccinator muscle involvement were recorded as the transducer was moved farther distally into the cheek space, which supported similar findings of Weinstein et al. (1963). 
In summary, the variance in tongue and lip pressures seen in "normal" individuals indicates that a wide range of maximum intraoral pressures occurs in the general population, making broad predictions about the effects of the perioral musculature difficult at best.

Weinstein et al. (1963) reported that the opposing forces and pressures exerted on the dentition from, principally, the tongue, cheeks, and lips can be self negating, such that a system of equilibrium between hard and soft tissues exists. Further conclusion were: (1) forces exerted on the teeth by the surrounding soft tissue may cause tooth movement much like orthodontic appliances, (2) each tooth may have more than one position of stable equilibrium within the system composed of the oral environment, and (3) differential forces, even of small magnitude, if applied over a considerable period of time, can cause changes in tooth position.

Proffit (1978) extended the work of Weinstein et al. (1963) by presenting an additional review of the subject of equilibrium. The results of Proffit's research identified four primary factors that create equilibrium between the dentition and the surrounding tissues:

1. intrinsic forces by tongue and lips,

2. extrinsic forces: habits, orthodontic appliances,

3. forces from dental occlusion, and

4. forces from the periodontal membrane.

Teeth characteristically exist in a state of equilibrium with the surrounding soft tissues. For posttreatment results to maintain stability, the resting pressures of the soft tissues will determine the final tooth positions.

Riedel (1976) acknowledged the importance of considering muscle balance when positioning teeth to gain lasting stability, but contended that establishing a harmonious muscle balance is almost impossible to do until growth and development have been completed. "The orthodontist moves the teeth into the relationships which he considers correct, but Nature eventually repositions them where they can best serve the patient" (Huckaba 1952, p349).

\section{Continued Physiologic Growth and Development}

About 1900, retention consisted of holding teeth in position until believed stable. This philosophy of stability changed when the Huning case of Dr. Angle demonstrated that anterior teeth can change axial inclinations during and after retention. This was the beginning of the realization that 
teeth continue to change axial inclinations as the children continue to complete their growth to adulthood (Steadman 1967).

These changes are rapid in childhood and early adolescence; by midadolescence, the pace has slowed but is still appreciable clinically; in adults, the astute clinical observer continues to detect changes, although they are of lesser degree and occur at a decreasing rate (Horowitz and Hixon 1969).

Craniofacial growth changes are variable among individuals. These changes do not stop in young adulthood, but have been shown to be a continuous process that occurs throughout adulthood and have an effect on the craniofacial skeleton (Behrents 1985, 1986, 2008). In a landmark follow-up study to the Bolton Study, Behrents (1985) was able to recall approximately 200 orthodontically untreated participants ranging in age from the late teens into the 80s. These individuals had been previously examined as children and young adults in the Bolton Study from 1928 through the 1970s. This allowed for documentation of long-term longitudinal data in the form of cephalometric head films on orthodontically untreated individuals followed in some cases from early adolescence to their mid-80s.

Behrents drew several conclusions about normal craniofacial growth in orthodontically untreated individuals. Behrents suggested that individuals experience significant "differential growth" within the craniofacial complex throughout adulthood due to increases in the size of their skeletal structures and changes in their shape. These changes in facial dimensions continue throughout adulthood, although less exuberantly than in adolescence (Behrents 1986). Overall, males grew more in later adulthood than females. The mandible increased in size in both sexes; however, in males, the mandible continue growth in a downward and forward direction, which produced a counterclockwise rotation of the mandible, while females exhibited more of a vertical change, causing the mandible to rotate in a clockwise direction. Maxillary incisors tended to upright in both males and females, while the mandibular incisors showed a consistent inclination in males, but a tendency for continued anterior inclination in females (Behrents 1986). Overbites did not change in either sex.

Harris (1997) also documented the significant skeletodental changes found occurring in untreated individuals over time. Based on longitudinal data available for 60 untreated adults, arch widths were found to significantly increase over time, while arch lengths significantly decreased. This altering of arch dimensions can produce a significantly shorter-broader arch form over time. In contrast to previous studies (Behrents 1985b; Perera 1987), incisor overjet and overbite were found to remain constant. 
Sinclair and Little (1983, p 114) assessed the dental casts of 65 untreated normal adolescents to determine "the nature and extent of the developmental maturation process of the normal dentition." A normal occlusion was defined as occurring in untreated individuals with dental and skeletal Angle Class I relationships. The sample was obtained from records of children in the Burlington Growth Center Study, Ontario, Canada. Six variables (irregularity index as described by Little in 1975, mandibular intercanine width, mandibular intermolar width, mandibular arch length, overbite, and overjet) were evaluated in the mixed dentition (9 to 10 years), early permanent dentition (12 to 13 years), and early adulthood (19 to 20 years). The results showed decreases in arch length and intercanine width; minimal overall changes in intermolar width, overjet, and overbite; and increases in Incisor Irregularity. It was also noted that females exhibited more severe dental changes than males.

More recently, Eslambolchi et al. (2008) documented the longitudinal dental changes in seen in 15 untreated children and 18 untreated adults who had been participants in the original Burlington Growth Research Project at the University of Toronto. In both groups, Little's irregularity index continued to increase 2.0 to $4.0 \mathrm{~mm}$ with age, while intercanine and interpremolar widths continued to decrease. Arch length also decreased up to $2.0 \mathrm{~mm}$ in the children and $1.0 \mathrm{~mm}$ in the adults over time.

Most orthodontic patients are treated in their adolescence, leaving ample opportunity for subsequent growth of the maxillary and mandibular complexes to move the teeth into unstable positions (Vaden, Harris and Gardner 1997; Gardner, Harris and Vaden 1998; Harris, Gardner and Vaden 1999). Conflicting reports exist in the literature as to the potential effects that continued craniofacial growth may have on incisor stability. Riedel (1960, p 187) admitted that, "Growth may be an aid in the correction of many types of orthodontic problems and it also may be of such character as to cause relapse of treated orthodontic patients."

Ricketts et al. (1972) reported that continued posttreatment growth may contribute to an increased pressure on the mandibular incisors as they are forced in a lingual direction with the advancement of the mandible. This occurs to a greater extent in patients whose incisors were tipped during treatment, as these teeth will have a greater tendency to be forced lingually. Similarly, Perera (1987) reported a relationship between mandibular growth and lower anterior crowding in untreated subjects $(n=29)$. Perera indicated that forward rotational growth in the mandible is closely related to lower incisor crowding that commonly occurs after the adolescent years. He suggested that as the mandible rotates forward, the lower incisors can become retroclined relative to the face. Similarly, Siatkowski (1974) conducted a longitudinal study that consisted of two 
sets of records for each of 18 untreated patients. He also noted lingual uprighting of the mandibular incisors in association with late growth changes, and concluded that anterior crowding can be influenced by posttreatment facial growth.

Shields et al. (1985) assessed 54 first-premolar extraction cases at least 10 years posttreatment to determine whether there were statistically significant correlations between cephalometric variables and the observed posttreatment change in Incisor Irregularity. They concluded that no cephalometric parameters, such as horizontal and vertical growth amounts were found to show any statistical association with changes in posttreatment mandibular anterior irregularity. In addition, pre- and posttreatment changes such as incisor position and facial growth were poor predictors of stability. Only a slight tendency was found for incisor inclination to return toward the pretreatment value during the postretention period.

According to Huckaba (1952), greater stability is seen in cases who were treated during periods of active growth and that growth accounts for many of the changes seen following orthodontic treatment. Based on this premise, Riedel (1960) suggested that corrections carried out during periods of growth are less likely to relapse; therefore, orthodontic treatment should be started at the earliest possible age. However, Riedel ( $p 185$ ) goes on to admit that, "There seems to be little positive evidence to substantiate this statement."

Harris et al. (1994) analyzed the effects of age on postorthodontic stability by comparing two samples five years out of treatment: 22 patients treated during adolescence and 23 patients treated during adulthood. All patients were treated by a single practitioner, had four premolars extracted, and were placed on identical retention protocols - maxillary Hawley and mandibular fixed lingual retainer. Posttreatment stability existed in both groups, but for different reasons. In the adult group, little posttreatment skeletal or dental change occurred. In the adolescent group, continued midface and mandibular growth occurred which compensated for any unfavorable mesial drift of the dentition. The conclusion drawn from this study was that, "Orthodontic corrections in adults were found to be at least as stable as those in the conventional adolescent patient" (p 25).

A retrospective longitudinal study was undertaken by Driscoll-Gilliland, Buschang and Behrents (2001) to compare the skeletal and dental changes seen in orthodontically treated patients with those in a comparable untreated group. The purpose was to evaluate the relationship between skeletal changes and mandibular incisor crowding. Results of the study were based on cephalograms and dental casts of 44 untreated subjects from the Broadbent-Bolton Growth 
Study and 43 orthodontically treated patients. Results indicated that growth continued in both groups, but growth in the vertical direction was twice that in the horizontal dimension for both groups. Treated patients were found to exhibit greater increases in overbite and overjet than did the untreated subjects. The authors concluded that there is significant growth, especially in the posterior and lower anterior facial height, beyond the typical age that orthodontic treatment is completed. Consequently, relapse may be promoted by the posttreatment growth patterns rather than treatment itself.

Fortunately, the pattern of growth in the majority of patients is seldom sufficiently deviant to product a marked effect on the dentition (Horowitz and Hixon 1969). Sinclair and Little (1983, p 114) concluded that, "Changes found in a sample of untreated normals were similar in nature but lesser in extent than postretention changes found in a sample of treated cases." Thilander (2000) suggested that the changes occurring after orthodontic treatment typically cannot be differentiated from the normal aging process. Therefore, the results of the previous research should be considered when attempting to attribute posttreatment changes to orthodontic relapse or simply as a part of the natural maturation process.

\section{Third Molars}

The effect that developing third molars play in posttreatment stability, most notably late mandibular incisor crowding, continues to be debated to present. Late crowding of the mandibular incisors, typically occurring in patients at about 16 to 18 years of age with ideal posttreatment orthodontic occlusions seems to affect all orthodontists at some time or another. The literature provides evidence that equally supports and refutes third molar influences on posttreatment stability.

Broadbent (1943) was an early researcher who contended that third molar effects on stability were insignificant. While Broadbent acknowledged the frequent posttreatment crowding of mandibular incisors, he concluded from twelve years worth of cephalometric evidence available from the Bolton research study, that late incisor crowding resulted from the failure of the facial skeleton to attain complete adult size and proportions following treatment. Broadbent also suggested that third molar impaction was an indication of abnormal facial development that also happened to be expressed in the incisor region. Steadman (1967) agreed with Broadbent that crowding due to third molar eruption is not a problem in children who have grown normally according to the Broadbent Standards. Steadman further suggested that there is an excessive anterior component of force that contributes to late mandibular incisor crowding that is 
created from impacted third molars resulting from inadequate mandibular growth

Richardson $(1980,1982,1989)$ devoted considerable effort to evaluating the impact that third molars might have on orthodontic treatment and late lower arch crowding. She demonstrated a significant forward movement of first molars between the ages of 13 and 17 years. This was correlated with the increase in lower arch crowding that occurred during the same period. There was no difference, however, in the forward movement of the first molar, in cases with or without impacted third molars. Richardson has also found that on average, there is a tendency for individuals with impacted third molars to have larger teeth, which could also contribute to late mandibular arch crowding. Evidence from Richardson's review article (1989) acknowledges a pressure from the back of the arch and the coincident presence of third molars in the cause of late lower arch crowding. However, Richardson stops short of attributing this pressure to the presence of the third molars and notes that this possible implication of third molars does not preclude the involvement of other causative factors.

In contrast, Bergstrom and Jensen (1960) examined 30 dental students who were found to be unilaterally missing one of their mandibular third molars. Greater crowding was found on the side with the third molar present as compared to the side with the third molar absent. Shanley (1962) contended that mandibular third molars exert little influence on crowding or labial inclination of mandibular anterior teeth. Fastlicht (1970) found no statistical correlation between the presence of third molars and lower incisor crowding. Sheneman (1969) noted greater stability was found in patients whose third molars were congenitally missing than in those whose third molars were present.

There is no consensus in the literature as to whether third molars contribute to instability of orthodontic results. Conclusions to be drawn from the literature indicate that if indeed the third molars do play a role in late mandibular incisor crowding, it is only a minor role, secondary to other factors, such as posttreatment changes in arch dimension and continued facial growth and development.

\section{Time Out of Treatment}

Some researchers claim that continuous significant and unpredictable relapse can be expected following orthodontic treatment (Little et al. 1981, 1988), others acknowledge continued changes in skeletal dimensions with age, but that rates of relapse diminish over time and long-term stability is possible (Sadowsky 
and Sakols 1982; Vaden et al. 1997; Gardner et al. 1998; Harris et al. 1999; Boley et al. 2003), while still others document differential rates of relapse for various locations in the dental arches posttreatment (Sadowsky et al. 1994; Al Yami et al. 1999).

Sadowsky et al. (1994) evaluated the stability of 22 orthodontic patients who were treated nonextraction a minimum of 5 years after all retention had been removed. The patients each had a maxillary Hawley retainer worn full time followed by nights only and a mandibular fixed lingual retainer in place for an average of 8.4 years prior to removal. During the posttreatment period, all variables measured (overbite, overjet, maxillary and mandibular Incisor Irregularity, and various interdental widths) showed relapse except for the expansion of the maxillary canines and bicuspids and mandibular Incisor Irregularity. This was attributed to the prolonged use of a mandibular fixed lingual retainer. Sadowsky et al. (1994) concluded that prolonged retention time may play an important role in long-term stability. This study promotes the importance of long-term retention.

Al Yami, Kuijpers-Jagtma and van't Hof (1999) conducted a longitudinal retrospective study to evaluate the stability of orthodontic treatment 10 years posttreatment. Dental casts of 564 patients taken 10 years posttreatment were analyzed using the Peer Assessment Rating (PAR) index. The PAR index evaluates the upper contact point displacement, lower contact point displacement, left buccal occlusion, right buccal occlusion, overjet, overbite, and centerline. Their results indicated that $67 \%$ of the achieved orthodontic treatment result was maintained 10 years postretention and that about half of the total relapse took place within the first 2 years after retention. All occlusal traits relapsed early on, but remained stable from 5 years onward, with one notable exception. The lower anterior contact point displacement showed a "fast and continuous increase" (p 300).

Harris, Gardner and Vaden (1999) evaluated long-term (10 to 15 years) postorthodontic craniofacial changes. Patients $(n=36$; mean age $=13$ years) were treated by one practitioner using standard Edgewise appliances and were recalled an average of 5.5 years after treatment and again 14.5 years after treatment. Although most skeletal linear dimensions increased from the end of treatment to first recall, presumably due to a continuation of adolescent growth, few dimensional changes were seen following the first recall period up to the second recall period. In fact, arch dimensions continued to improve following active treatment. They concluded that maxillary and mandibular growth continued into early adulthood but that vertical maxillary growth continued through the late recall period. Most returns toward pretreatment status occurred soon after orthodontic treatment. Because the majority of growth during the 
study occurred during the active phase of treatment, the results suggest that if relapse does occur, it is most likely to occur soon after treatment and can be expected to diminish thereafter. Long-term postorthodontic changes were interpreted as a combination of orthodontic relapse and normal growth and aging processes.

At the University of Washington, Little, Riedel, and Årtun (1988) evaluated 31 four-premolar extraction cases from a previous study (Little, Wallen, and Riedel 1981) at a minimum of 20 years posttreatment. Variables such as Angle's classification, patient age at the beginning of treatment, sex, overbite, overjet, arch width, arch length were analyzed at each examination. The studies evaluated mandibular anterior alignment. The secondary goal was to describe treatment and posttreatment changes and search for predictors and associations of stability. Results indicated that crowding continued to increase during the 10- to 20-year postretention phase. The authors concluded that, "Cases responded in a diverse unpredictable manner with no apparent predictors of future success when considering pretreatment records or the treated results" ( $\mathrm{p}$ 423).

\section{Severity of Pretreatment Malocclusion and Posttreatment Stability}

Physiologic recovery has been described as, "The type of posttreatment changes that represent a rebound or reversion toward the original malocclusion" (Horowitz and Hixon 1969, p 4). Example of changes include: rotations, lower anterior crowding, collapse following arch expansion, molar relationship changes, and recurrence of overbite (or open-bite) and overjet. It could be assumed that cases who demonstrate an increased severity of pretreatment malocclusion will also exhibit a greater degree of posttreatment relapse.

A recent study at the University of Washington involving 86 patients was undertaken to identify factors associated with stability (Ormiston et al. 2005). The sample was divided into 2 groups according to whether their treatment results were deemed stable or unstable based on the PAR index and irregularity index. The authors found the initial severity of malocclusion to be negatively correlated with posttreatment stability. They concluded that patients with more severe index scores before treatment tended to be less stable.

Incisors that are labially inclined prior to treatment tend to exhibit less long-term crowding (Sanin and Savara 1973; Gilmore and Little 1984). Class II, division 1 malocclusions have been reported to exhibit around twice as much relapse in overbite correction as Class II, division 2 malocclusions according to Bresonis and Grewe (1974), who analyzed 53 orthodontically treated cases at 5 
years postretention. Årtun et al. (1996) found that narrow pretreatment intercanine width and increased pretreatment Incisor Irregularity associated with Class II, division 1 malocclusions were significant predictors of relapse in the mandibular incisors. However, other studies (Bishara et al. 1973; Elms et al. 1996) have found no greater relapse associated with Class II division 1 malocclusion cases when compared with other types of malocclusions.

\section{Prevention of Post-Orthodontic Relapse}

The proposed rationale for retaining teeth in their treated position is: to allow time for periodontal and gingival reorganization; to minimize changes from physiologic growth; to permit neuromuscular adaptation to the corrected tooth position; and to maintain unstable tooth position, if such positioning is required for reasons of compromise or esthetics (Blake and Bibby 1998). Controversy involves questions such as, "Just how should the teeth be retained," and "For how long a period of time should they be retained?" The following section describes various methods of retention and reviews opinions regarding recommended retention periods.

\section{Types of Mechanical Retention}

The purpose of posttreatment retention, according to Angle (1907, p 263), is that "After malposed teeth have been moved into the desired position they must be mechanically supported until all the tissues involved in their support and maintenance in their new positions shall have become thoroughly modified, both in structure and in function, to meet the new requirements." Proffit (1993) has described various forms of mechanical retention as removable, fixed, passive, or active, mechanical retention can generally be broadly classified based on whether or not the retainer is intended to be taken in and out of the mouth by the patient. Both fixed and removable forms of retention continue to be popular means of maintaining postorthodontic treatment results. Since 1925, the standard maxillary retaining device has been the removable Hawley retainer, while in the lower arch, the cemented canine-to-canine retainer has been popular for possibly a longer period of time (Muchnic 1970).

\section{Removable Retention}

Of the current methods, the traditional Hawley-type appliance (Hawley 1919 ) is probably by far the most commonly employed. This removable appliance can be fabricated for both the maxillary and mandibular arches and 
consists of an acrylic base that typically fits against the lingual surfaces of the teeth with round $0.028^{\prime \prime}$ or $0.030^{\prime \prime}$ stainless steel wire that is incorporated into the base and fits over the occlusal aspect of certain teeth for retention, then continues as a "labial bow" that follows the posttreatment facial contour of the anterior teeth in an effort to maintain the orthodontic result. Patients are typically advised to wear their Hawley retainers full time or as much as possible during the first six months posttreatment, followed by indefinite nighttime only wear after this time.

Vacuum-formed retainers (VFRs) are another common type of appliance used for retention. These acrylic retainers are fashioned out of clear 0.030 " acrylic coping material (Paulson 1992) that is heated and then vacuum-formed onto plaster casts of the patients' treated dentition. The resultant trays are then trimmed to provide 1 to $2 \mathrm{~mm}$ buccal and 3 to $4 \mathrm{~mm}$ lingual extensions past the gingival margin such that they fit over the occlusal surfaces of the teeth, including the most distal tooth (Rowland et al. 2007). These are commonly clear and are can be fabricated for the maxillary and mandibular arches. Instructions for use are similar to those of Hawley retainers.

Orthodontists may use a positioner immediately following comprehensive orthodontic treatment in an effort to achieve three-dimensional control of posttreatment tooth position. Kessling (1945) described an elastomeric positioner to allow for minor tooth movements, space closure, and overbite correction if worn correctly by patients following orthodontic treatment. Nowadays, this rubber-based appliance is custom made to the patient's posttreatment occlusion and centric relation, but adjusted to fine-tune the occlusion by allowing minor changes to occur as the patient functions in it. This type of control "helps the teeth settle into the most ideal functional and aesthetic relationships without losing the centric relation position of the mandible" (Wasson 1988, p 31). Following this "settling" of the teeth, the positioner can continue to serve as a traditional removable retainer if worn on a limited basis.

The logic of removable retention is that it allows functional integration of teeth into their new positions and does not totally protect them from the posttreatment forces that they will be influenced by for the remainder of their service (Waldron 1942). Waldron (1942) further suggested that because such forces are natural forces, their effect is not only enduring, but functionally essential, and he suggested that a removable retainer that permits maximum functional freedom to the denture, while providing minimal retentive restraint, is the best form of appliance.

The primary advantage of a removable retainer is that the patient (and orthodontist) maintains the ability to easily remove it for cleaning and 
adjustment, if necessary. This ability to be removed also serves as the primary disadvantage as well. The ability of the removable retainer to maintain tooth position is completely dependent on patient compliance and wear. Lack of patient compliance regarding instructions for use can result in compromised posttreatment stability.

\section{Fixed Retention}

Edward H. Angle (1907) was one of the first to advocate the use of cemented bands in his retentive appliances. Today, the most commonly used form of fixed retention is some variant of a round archwire that is bonded to the lingual surfaces of the maxillary central incisors or the lingual surfaces of the mandibular anterior teeth, typically from canine to canine (Zachrisson 1977, 1978). Occasionally, the first and second premolar teeth are included if these teeth had been extremely malpositioned at pretreatment or to prevent mesial tipping of these teeth or the opening of spaces in extraction cases (Rosenstein and Jacobson 1971). The purpose of mandibular canine-to-canine fixed retention is "(1) to prevent incisor recrowding, (2) to hold the achieved lower incisor position in space, and (3) to keep the rotation center in the incisor area when a mandibular anterior growth rotation tendency is present" (Zachrisson and Büyükyilmaz 2005, p 622).

Wires typically used are either a braided or a solid section of wire. Currently, the most commonly used solid wires are either a round 0.032 inch stainless steel or 0.030 inch gold-coated wire (Zachrisson 1997), bent and sandblasted on the ends for improved retention for bonding (Zachrisson and Büyükyilmaz 2005). These solid wires are usually bonded only to the lingual surfaces of the canines with light cured composite. This form of retention allows rigid fixation of the teeth, thus prohibiting the potential physiologic response of the teeth to extraneous forces exerted on them.

The use of a braided wire for bonded retention has recently become popular. Proponents of the braided type wire claim that its flexibility allows for some physiologic tooth movement in response to the surrounding forces, while tooth position is maintained (Dahl and Zachrisson 1991; Bearn 1995). Typically, because of the added flexibility of these wires, clinicians are able to bond all anterior teeth to it, including the canines. Atack et al. (2007) analyzed two groups of 29 patients at least one year post debonding. One group had bonded lower canine to canine multistrand retainers placed following debonding, while the other group received lower Hawley-type retainers. Based on Little's irregularity index, relapse was seen in both groups, but no statistical difference was found in 
the rate of relapse between fixed, braided archwire retainers and standard Hawley-type removable retainers.

The logic of fixed retention is to provide a constant, rigid fixation of the mandibular anterior teeth until the supporting structures are able to adapt to the treatment changes, independent of patient compliance. Angle (1907) stated that the posttreatment dentition should be placed in rigid fixed retention for as long as possible. Contrary to Angle's perspective was the opinion of Markus (1938) who assumed from his search of the literature that teeth must not be fixed following active treatment because this implies that occlusion is static. The positions of the teeth may change immediately, or long after the mechanical retentive appliances are removed.

The primary advantage for using fixed means of retention is that it cannot be removed by the patient, which obviates any patient compliance issues. However, use of fixed retention requires periodic observation, maintenance and accountability for presence of the appliance. Placement of fixed retainers has been considered by some to be time-consuming and technique sensitive (Zachrisson 1977; Lee 1981; Dahl 1991), and, for some individuals, they can be difficult to manage and maintain, encouraging unwanted tooth movement and accumulation of plaque and calculus (Heier et al. 1997; Atack et al. 2007; Katsaros et al. 2007; Pandis et al. 2007). However, other evidence (Årtun 1984; Årtun et al. 1987; Årtun et al. 1997) suggests that there are "few such long-term problems" (Atack et al. 2007, p 954). Issues have also arisen as to whose responsibility it is to manage the long-term monitoring of the fixed retainer: the orthodontist, general dentist, or hygienist.

Current indications for the use of mandibular canine-to-canine fixed retention include (1) nonextraction cases in which slight increases in arch length or mandibular intercanine width have been produced (Riedel 1976), (2) patients with pretreatment diastemas or severe incisor crowding or rotations, (3) patients with a pretreatment flat functional occlusal plane or open bite (Zachrisson and Büyükyilmaz 2005), (4) Class II patients with a rotation center in the premolar area (Björk's anterior rotation Type III), (Björk 1965), and (5) patients with a Class III growth tendency (Zachrisson and Büyükyilmaz 2005). Riedel (1976) stated that mandibular fixed retention successfully stabilizes the lower anterior segment until one has resolved the problem of the third molar and resolved problems related to growth changes. 


\section{Duration of Retention}

The premise is essentially that if the diagnosis is correct, and treatment mechanics are applied as efficiently as possible, then retention is merely the continuation of mechanotherapy for a reasonable period of time (Rosenstein and Jacobson 1971). However, what constitutes a reasonable period of time?

According to Muchnic (1970), the answer depends on the type of case treated, the age of the patient, patient expectations from orthodontic treatment, and the orthodontist's expectations of treatment. Currently, there is no consensus as to a recommended length of time that teeth should be retained posttreatment and opinions regarding the length of retention are largely dependent on testimonial data based on clinical experience of various orthodontists. Recommendations in the literature vary widely from no retention (Mershon 1927; Strang 1949; Andrews 1972) to permanent retention (Riedel 1960; Horowitz and Hixon 1969; Little et al. 1988). Because of Reitan's work (1967) that showed that teeth that have been moved during orthodontic treatment tend to return toward their former positions, Riedel (1960) contended that all patients must be thoroughly evaluated to determine what type, if any, and for how long retention procedures should be undertaken.

Rosenstein and Jacobsen (1971) offered four options for individualized patient retention. The first is to use retention for a short a period of time (4 to 6 months). This would be reserved for patients who exhibited mild malocclusions initially and only minimal tooth movement was required for correction. A second option is retention for at least half of the active treatment time. This seems to be merely a general rule of thumb that can be applied to the average treated case. A third option allows for retention to be in place "until the problem of the third molars has been resolved" (p 331). This option is based on the controversial literature that ascribes late incisor crowding to the presence of the third molars. The fourth option offered is to allow for retention to remain in place of as long as possible or as long as the patient will allow. This assumes that the retainer is esthetically acceptable and comfortable to the patient, oral hygiene is not a problem, and that the patient will maintain regularly scheduled recall visits.

Reitan (1967) reckoned that, it takes, on average, a minimum of 232 days for periodontal fibers around the teeth to remodel to the newly acquired tooth position. However, even where teeth have been maintained in position for more than this length of time, cases commonly relapse over the long term (Little, Wallen, and Riedel 1981; Little, Riedel, and Artun 1988). According to Paulson (1992), bonded retainers should be left in place for only 3 to 4 years, except in cases involving adolescent patients still undergoing their adolescent growth spurt, because any longer than 4 years exceeds the recommendation from 
Reitan's work $(1959,1969)$ regarding the time necessary for elastic fiber reorganization. Tweed $(1944,1952)$ suggested that retainers should be worn for at least 5 years in most cases. Research conducted by Harris, Vaden, and Gardner $(1997 ; 1998 ; 1999)$ has indicated that most "relapse" occurs soon after treatment in orthodontically treated patients and untreated subjects. Other studies (Al Yami et al.1999; Kuijpers-Jagtman 2002) also show decreases in the rates of relapse over time; however, with a notable exception in the mandibular incisors.

Based on long-term longitudinal data, Behrents (1986) concluded that because continued growth occurs in individuals throughout life, orthodontists have incomplete control of their results and possibly should not accept indefinite responsibility for a perfect result because there are no biologic guarantees. His recommendation is that retention should be in place until the mid-20s for males and the early 20s for females. However, in his review of the literature, Bearn (1995) found that bonded fixed retainers are now in common use for long-term esthetic retention and Zachrisson and Büyükyilmaz (2005) claimed that extended retention periods (up to 10 years) are now recommended by most clinicians (Behrents 1989; Gorman and Smith 1991; Sadowsky et al. 1994; Zachrisson 1997). Renkema et al. (2008) stated that the 3-3 mandibular lingual retainer was effective in preventing relapse in the mandibular anterior region in most patients, and Booth et al. (2008) reported that long-term retention of mandibular incisor alignment is acceptable to most patients and quite compatible with periodontal health.

\section{Additional Retention Methods}

Orthodontists have many options other than mechanical retention available to help diminish the effects of relapse on treated cases. Orthodontists routinely remove small amounts of tooth structure from the contact areas of particular teeth. In a procedure referred to as reproximation or interproximal reduction, roughened metal strips or narrow discs are used to flatten the interdental contacts and remove small amounts of adjacent tooth surfaces. It is supposed that a flatter contact is more retentive than a point contacts because there is more surface area available to prevent slippage of the contact area (Boese 1980). Interproximal reduction is useful in situations where tooth size discrepancies, as described by Bolton (1958), exist. Selective removal of interproximal tooth structure can help to accommodate certain interarch tooth size discrepancies, allowing for improved occlusion and incisor coupling; potentially facilitating a more stable posttreatment result. 
The reduction in tooth structure of adjacent tooth surfaces also allows for an increase in available arch space to correct minor crowding without the subsequent proclination of the incisors (Boese 1980). Bolton (1962) has suggested that up to approximately $3.5 \mathrm{~mm}$ of enamel can be removed from the six mandibular incisors as needed, and Riedel (1976) stated that potential problems are not likely to occur if $0.5 \mathrm{~mm}$ of enamel is removed from each of two neighboring anterior teeth. Currently, practitioners looking to avoid extractions to relieve tooth-arch discrepancies rely on some combination of arch expansion and interproximal reduction. This method has become an increasingly popular method to relieve mild to moderate crowding in the current literature. However, Lindauer (1997, p 31) warns that, "Unless a tooth size discrepancy exists prior to treatment, tooth size reduction will have to be accomplished equally in both arches if normal interarch dental relationships are to be attainable. Teeth that are flared or anteriorly positioned and accompanied by significant amounts of intraarch crowding, more strongly suggest an extraction decision."

Posttreatment surgical approaches have also been taken as a means of stabilizing orthodontic results, especially following the correction of tooth rotations. Although Jackson (1904) did not use the term "fiberotomy," he was the first to mention the procedure when he suggested that "after they [teeth] have been rotated as far as described, the soft tissue should be separated from the neck of the tooth and allowed to reunite in the new location, depending on the cicatrix thus formed to prevent their retrograde movement" (Kaplan 1988, p 326).

In an effort to follow up on the Reitan research $(1959,1967,1969)$ and to help understand the reactions of the periodontal tissues to orthodontic rotations of teeth, Edwards (1968) studied histologic tissue sections taken from seven dogs which had teeth experimentally rotated with conventional orthodontic appliances. Edwards (1970) described a surgical procedure in which the supracrestal fibrous attachment around the circumference of a tooth was severed in order to prevent rotational relapse. Boese (1980) proposed "circumferential supracrestal fiberotomy" in conjunction with "reproximation" (interproximal enamel reduction). He documented enhanced stability of the mandibular incisors at between 4 and 9 years posttreatment in 40 patients who exhibited significant mandibular incisor crowding at pretreatment. Edwards (1988) found that rotational relapse was prevented more consistently than labiolingual relapse, and that the surgical procedure was more effective in reducing relapse in the maxillary arch than in the mandibular arch. However, other authors have found unpredictable tooth movement can still occur following circumferential supracrestal fiberotomy (Rinchuse et al. 2007) while still others find it difficult to draw any definitive conclusions about the effectiveness of circumferential supracrestal fiberotomy (Riedel and Brandt 1976; Littlewood et al. 2006). 
Conflicting evidence regarding third molar effects on posttreatment mandibular incisor crowding has previously been discussed. In an effort to negate any potential impact on stability, third molars are routinely extracted following orthodontic treatment. Retention, in these cases is kept in place until the problem of the third molars has been resolved (Rosenstein and Jacobsen 1971).

Overcorrection is another technique aimed at minimizing relapse of teeth. This is commonly considered during treatment of rotated teeth. The thought is that by over-rotating the offending tooth with the notion that the there will be some tendency for the tooth to rotate back towards its former position. However, there is little evidence to indicate that over rotation is successful in preventing this type of relapse (Riedel 1960; Edwards 1970) and the amount of overcorrection needed to achieve stability is unknown.

Another treatment protocol involves the paralleling of tooth roots and evenly spacing them from each other during the finishing stages of orthodontic treatment. Rosenstein and Jacobson (1971) claim that if specific care is used to parallel the roots of the teeth adjacent to the extraction sites, then relapse becomes less of a problem. Based on clinical experience and research conducted on over 10,000 of his completed cases, Alexander (2008) contends that proper bracket placement will allow for proper root positioning, i.e., the roots of the anterior teeth should be divergent and the roots of teeth adjacent to extraction sites should be parallel to each other.

\section{Summary}

The current consensus regarding the retention of posttreatment results can be found in the six conclusions drawn by Blake and Bibby (1998) in their literature review of retention and stability:

1. The patient's pretreatment lower arch form should be maintained during orthodontic treatment as much as possible.

2. Original lower intercanine width should be maintained as much as possible because expansion of lower intercanine width is the most predictable of all orthodontic relapse.

3. Mandibular arch length decreases with time. 
4. The most stable position of the lower incisor is its pretreatment position. Advancing the lower incisors is unstable and should be considered as seriously compromising lower anterior posttreatment stability.

5. Fiberotomy is an effective means of reducing rotational relapse.

6. Lower incisor reproximation enhances posttreatment stability.

\section{Significance of Research}

Many orthodontists use fixed retention appliances following orthodontic treatment as a means of maintaining treatment results. These appliances incorporate the use of either a braided or solid, round stainless steel wire bonded to the lingual surfaces of the maxillary central incisors or mandibular canines. The purpose of this appliance is to provide a retentive appliance that will allow for semi-rigid fixation of the anterior segment of the dentition without having to rely on patient compliance.

Evidence supporting the need for fixed retention is conflicting. Studies conducted at the University of Washington by Little and coworkers (1981 and 1988) concluded that: long-term stability following mandibular anterior alignment was unpredictable; anterior crowding continued during the 10 and 20 year recall period; future stability of cases was unpredictable; and the majority of patients displayed unsatisfactory incisor alignment following retention. Thus, it is the contention of Little that fixed retention is the only way to maintain longterm post-orthodontic stability. However, Andrews (1972, p 307) stated that, "Lower teeth need not be retained after maturity and extraction of the third molars, except in cases where it was not possible to honor the musculature during the treatment and those cases in which abnormal environmental or hereditary factors exist." Furthermore, Boley (2007) concluded that, based on his review of the literature, stability is a reasonable and predictable goal following orthodontic treatment as long as certain parameters are met.

Some practitioners advocate fixed retention only for a temporary period of time following treatment, such as 2 to 3 years. This notion is supported by the research of Al Yami et al. (1999) who evaluated the dental casts of 1,016 patients for long-term stability of orthodontic treatment using the Peer Assessment Rating index. About half of the total relapse took place in the first two years following retention, and all aspects of occlusion relapsed gradually over time but became stable after 5 years with the exception of the mandibular incisors. The presence of fixed retention had a significant impact on relapse at 5 and 10 years 
postretention as shown by a reduction of the PAR index by 3.6 points and 4.6 points, respectively, when compared to cases without fixed retention.

Therefore, because instability of the orthodontic treatment result occurs to some extent in practically every patient (Joondeph 2005) and the mandibular anterior segment of the denture is the least predictable and most common site of relapse, an important focus of research continues to be Incisor Irregularity of the mandibular denture segment over time. Orthodontists need evidence-based studies to support their treatment decisions and to provide authoritative information concerning the etiology of relapse as well as the clinical rationales to minimize it. The present research will help to establish if there is any statistical difference in relapse at long-term recall between patients who were provided temporary fixed retention compared with those who received only removable retention. 


\section{CHAPTER 3. MATERIALS AND METHODS}

\section{Sample Selection}

Data consisted of orthodontic records that included facial photographs, panoramic and cephalometric radiographs, and plaster casts of 236 individuals, all of whom had received comprehensive orthodontic treatment. Records were available at three time intervals for each patient: pretreatment (T1), end of treatment (T2), and at long-term posttreatment recall examination (T3). The records were collected by Dr. George S. Harris (Menominee, Michigan) from private practitioners across the North American continent who use standard Edgewise mechanics and were members of the Charles H. Tweed International Foundation. With financial support from the Charles H. Tweed International Foundation, the Tweed Foundation Research Committee was formed. The goal of the committee was to have every member provide ten sets of records, including pretreatment, retention, and 10 year post-treatment recall records (Harris 1985). Members were asked to submit records of cases that were a minimum of 10 years out of treatment, regardless of treatment outcome. This would allow the establishment of a broad based sample to describe the quality of the cases and to allow members to learn from treatment failures as well as successes. Data files containing these cases were created by Dr. George S. Harris and Dr. James L. Ferguson. The Foundation's plan was to evaluate the long-term orthodontic stability of people treated with standard Edgewise mechanics. These records (dental casts and cephalograms) were digitized and measured by Donna Niemczyk with guidance from George Harris and James Ferguson using DentoFacial Planner ${ }^{\circledR}$ software (DentoFacial Planner, Toronto, Ontario, Canada).

Cases in the present study $(\mathrm{n}=166)$ were a subset of the larger series collected by the Tweed Foundation Research Committee. We used just those cases where, from inspection of the posttreatment casts, there was or was not a fixed lingual retainer - that is, the posttreatment retention protocol consisted either of (A) a maxillary Hawley retainer in combination with either a fixed mandibular canine-to-canine or first premolar to first premolar retainer or (B) maxillary and mandibular Hawley retainers. Of the original data from 236 longterm recall study cases received from Dr. George Harris and Dr. James Ferguson (Figure 3-1), 51 cases were excluded because the recall records did not meet the minimum 10 years posttreatment criterion, 6 cases were found to still have fixed retainers in place at the recall appointment, 8 cases did not have recall records present, 1 case was treated by serial extraction only and received no banded treatment, and 4 cases were excluded due to incomplete records or uncommon extraction patterns. 


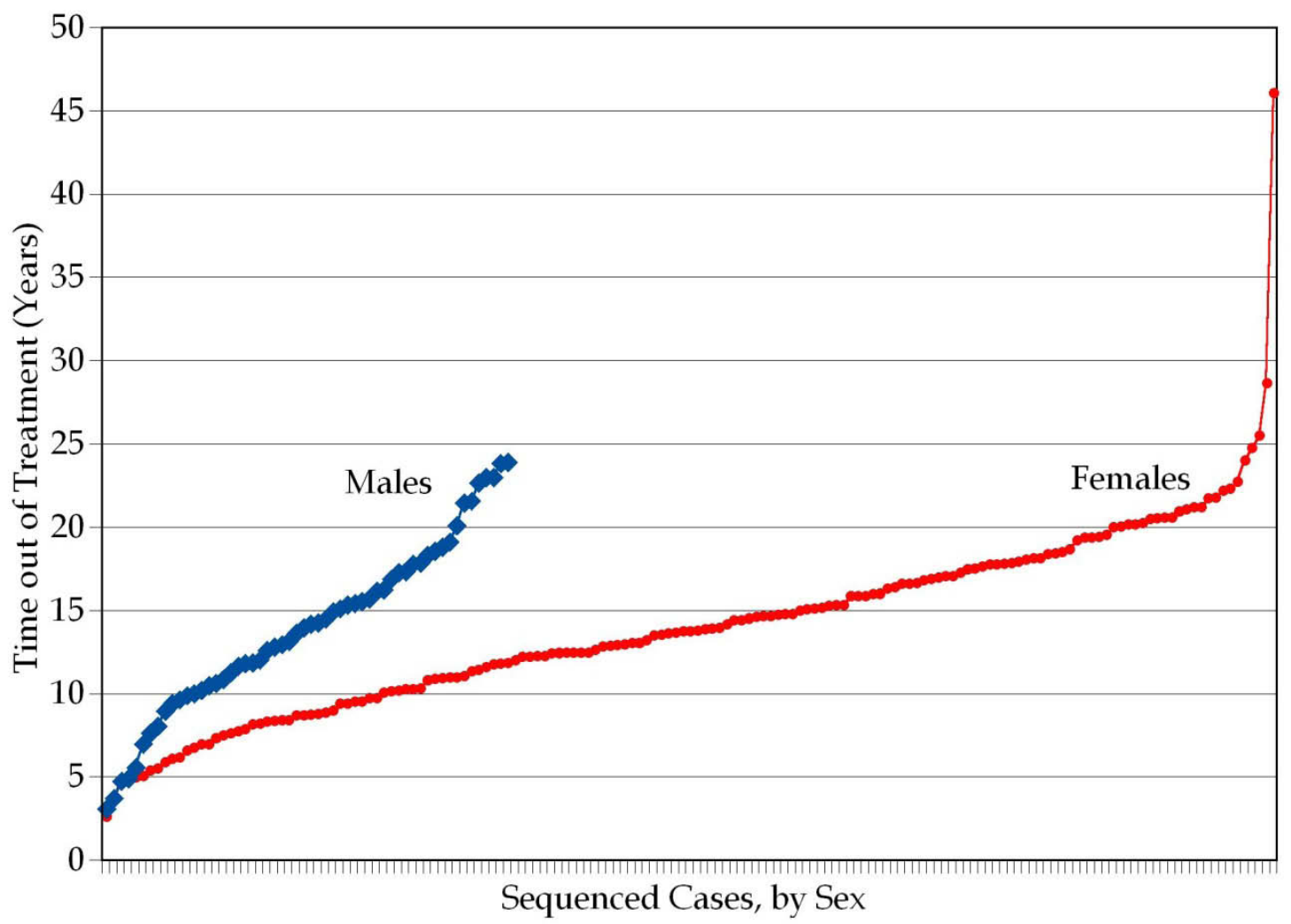

Figure 3-1. All cases $(n=236)$ available in the Tweed Foundation Study. Cases are arranged by their time out of treatment (i.e., time from T2 to T3). All cases are plotted, but only those $(n=166)$ that were a minimum of 10 years out of treatment were analyzed in the present study. There were appreciably more women than men in the study. Except for 3 women with records more than 25 years out of treatment, the rest of the sample analyzed here was between 10 and 25 years out of treatment. This subset of the Tweed Foundation Study analyzed here consisted of 43 men and 123 women. 
The present sample consisted of the remaining 166 cases (Table 3-1). All patients were American whites, with pretreatment ages between 8.26 and 43.36 years. Patients at T3 were a minimum of 10.0 years out of treatment. All fixed retainers were removed approximately $2-3$ years after posttreatment. Following removal, no mandibular retention was used, and maxillary retainer wear was left up to the discretion of the patient.

\section{Sample Description}

One part of the sample consisted of 69 subjects ( 23 males; 46 females) who had been placed in a fixed mandibular canine-to-canine retainer and a maxillary removable retainer (traditional Hawley-type) at the end of active treatment. The other part of the sample consists of 97 subjects (20 males; 77 females) who were placed in maxillary and mandibular removable retainers (traditional Hawleytype). Both groups exhibit a low proportion of males because of men's lower uptake of orthodontic services plus their reluctance to return for a recall examination.

Pretreatment (T1), posttreatment (T2), and recall (T3) records were available for all of these 166 cases (Figure 3-1). Mean initial examination age for the sample was 13.94 years (Table 3-1). Posttreatment records were taken at a mean age of 16.89 years, with the total treatment time for the sample averaging 2.94 years. The interval from the posttreatment to recall examination was on average 16.03 years for the sample, with an average recall age of 32.92 years.

\section{Description of Data Entry}

Steps in the cast analysis (conducted by the Tweed Foundation research group) were this: Landmarks on each cast were marked with a soft lead pencil. Vertical lines were drawn on the maxillary cast on the buccal surfaces of the right and left first molar from the mesial buccal cusp tip to the gum line. On the mandibular cast, a vertical line was drawn one millimeter distal to the mesiobuccal groove on the buccal surface of the first molars (Figure 3-2).

Holding the casts in occlusion (maximum interdigitation), the Left Molar Correction and Right Molar Correction values were measured, from the marks made as just described. This method produces a value $1.0 \mathrm{~mm}$ greater than commonly obtained (Baume et al. 1973; Harris and Corruccini 2008). The difference occurs because the Tweed Foundation teaches over correction of Class II cases to about $1 \mathrm{~mm}$ past traditional Angle Class I occlusion. That is the 
Table 3-1. Descriptive statistics for chronologic ages at the three examinations $(\mathrm{n}=166)$.

\begin{tabular}{lrrrcc}
\hline \multicolumn{1}{c}{ Statistic } & $\begin{array}{r}\text { Start } \\
\text { Age }\end{array}$ & $\begin{array}{r}\text { End } \\
\text { Age }\end{array}$ & $\begin{array}{c}\text { Recall } \\
\text { Age }\end{array}$ & $\begin{array}{c}\text { Time in } \\
\text { Treatment }\end{array}$ & $\begin{array}{c}\text { Time } \\
\text { to Recall }\end{array}$ \\
\hline Median & 12.84 & 15.76 & 32.15 & 2.71 & 15.25 \\
Mean & 13.94 & 16.89 & 32.92 & 2.94 & 16.03 \\
SD & 4.68 & 4.42 & 5.70 & 1.09 & 4.48 \\
SEM & 0.36 & 0.34 & 0.44 & 0.08 & 0.35 \\
$\mathrm{~L}_{1}$ & 13.23 & 16.21 & 32.04 & 2.77 & 15.34 \\
$\mathrm{~L}_{2}$ & 14.66 & 17.56 & 33.79 & 3.11 & 16.72 \\
\hline
\end{tabular}




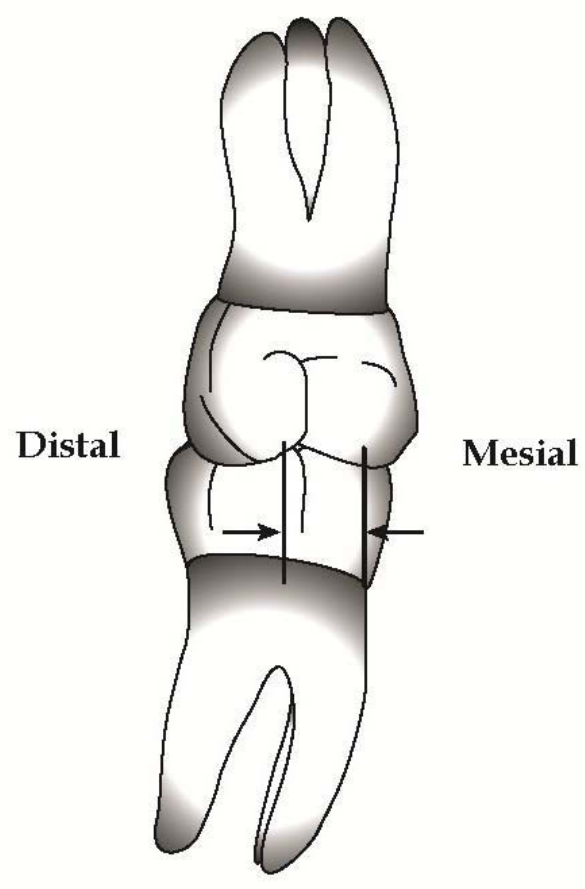

Figure 3-2. Illustration of how molar correction was measured.

Molar correction is defined in the present study as the distance between the mesiobuccal cusp of the maxillary first molar and the line one millimeter distal to the buccal groove of the mandibular first molar. When the cusps of the maxillary first molar are locked tightly in the fossae of the mandibular fossae in a perfect Class I molar relationship, the maxillary molar mesiobuccal cusp is $1 \mathrm{~mm}$ distal to the buccal groove. (This one-millimeter addition is not usually included in measures of buccal segment relationship; see Baume et al. 1973; Harris and Corruccini 2008). 
treatment goal at the end of treatment. The expectation is that the case will settle into a stable Class I occlusion.

The occlusal surfaces of the casts were photocopied at $200 \%$ of natural size. From this photocopy, 41 anatomical landmarks were digitized (Figure 3-3). The procedure was performed on three sets of dental casts. Landmarks were identified on the dental casts for (T1), (T2), and (T3). Photocopies of the casts were then digitized using DentoFacial Planner ${ }^{\circledR}$ software. Measurement reliability of the sample lies in the fact that a single technician, Ms. Donna Niemczyk, digitized all the records.

Five lines were drawn on the mandibular arch photocopy (Figure 3-4). Lines were drawn along the left and right buccal segments centered over the alveolar ridge. The point of intersection of these lines, anterior to the incisors, forms the Buccal Segment Angle. A line bisecting this angle was drawn, as well as a line connecting the left and right canine cusp tips, and left and right first molar buccal grooves. On the maxillary casts, three lines were drawn, namely a line from the incisive papilla to the midline mark between the molars, a line between maxillary first molar mesial buccal cusp tips crossing the gum lines, and a line connecting the maxillary canine cusp tips.

Using the lines drawn on the casts, six mandibular arch landmarks were identified (Figure 3-5):

1. Midline point: The intersection of the buccal segment angle bisector line with a perpendicular line crossing the incisal edge of the more protrusive central incisor.

2. Anterior-midarch dividing points (left and right): This coincides with the contact points of the mandibular canines first or second premolars in a wellaligned and stable mandibular dental arch. They are centered over basal bone and are slightly buccal to the buccal segment line.

3. Occlusal depth points (left and right): This is the line drawn across the buccal segment line corresponding to the occlusal depth value (times the photocopy magnification factor) distal to the first molar. The procedure is repeated on the other side.

4. H point: A point on the midline of the mandibular occlusal plane at the intersection of the buccal segment bisector with a line drawn tangent to the distal of the more distal first molar and perpendicular to the buccal segment bisector. 

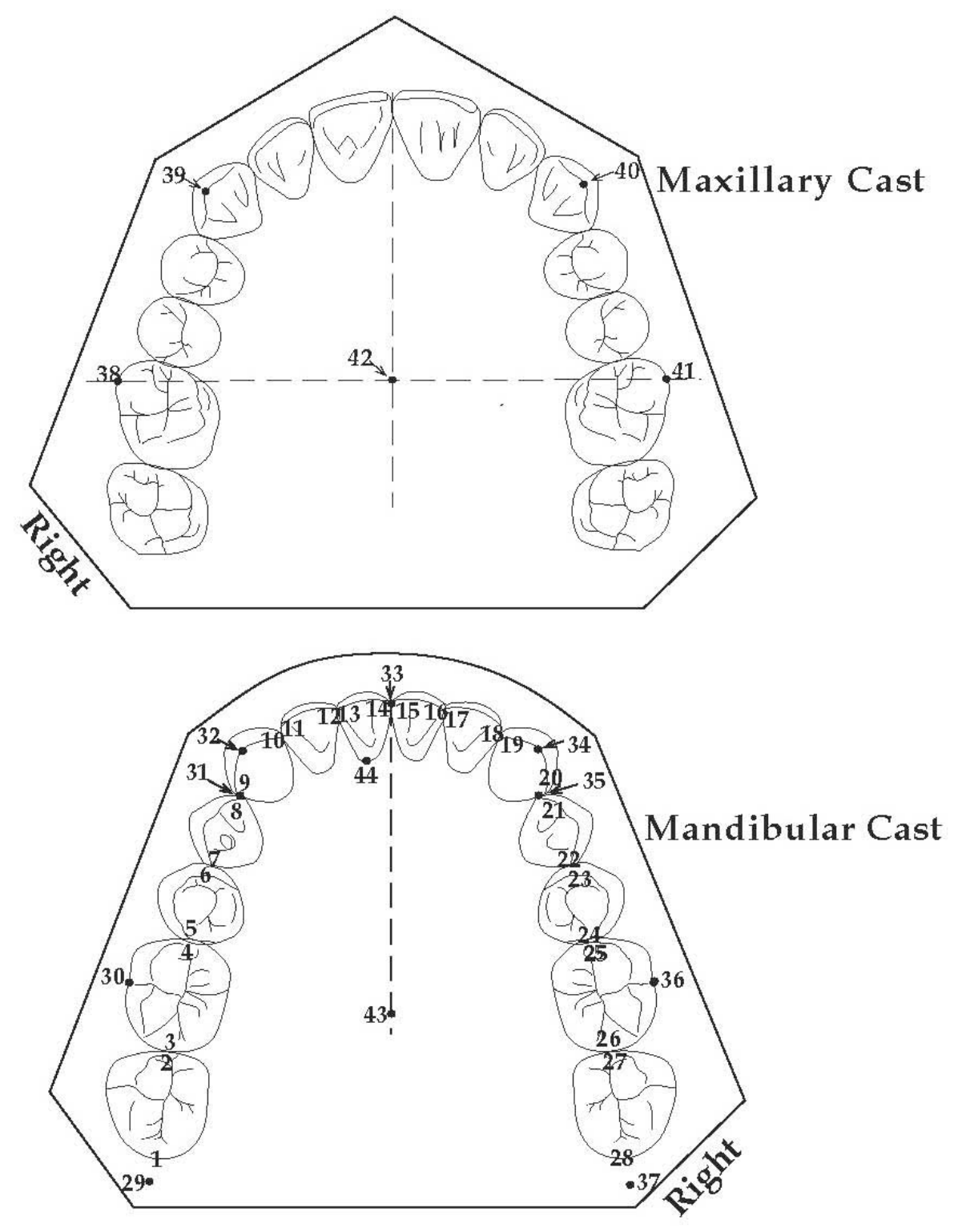

Figure 3-3. Illustration showing digitized landmarks.

The mesial and distal contact points of each tooth were digitized, along with canine cusp tips, first molar gingival line points, left and right divide points, left and right occlusal depth points, and the midline point. There were 39 mandibular landmarks and 5 maxillary landmarks, for a total of 44 fiducial points. 


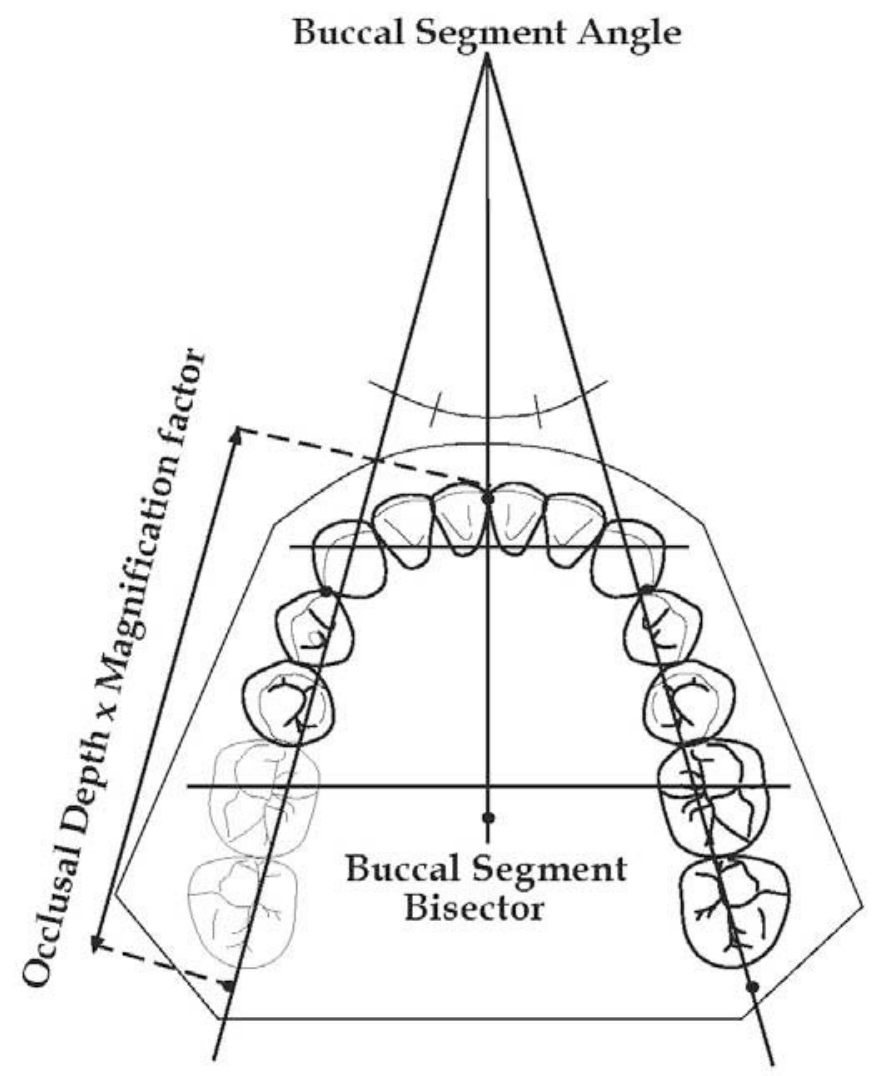

Figure 3-4. Illustration of how the buccal segment angle is constructed. These lines were constructed on the photocopy of each cast. The buccal segment lines intersect, forming the buccal segment angle. The buccal segment bisector bisects this angle. 


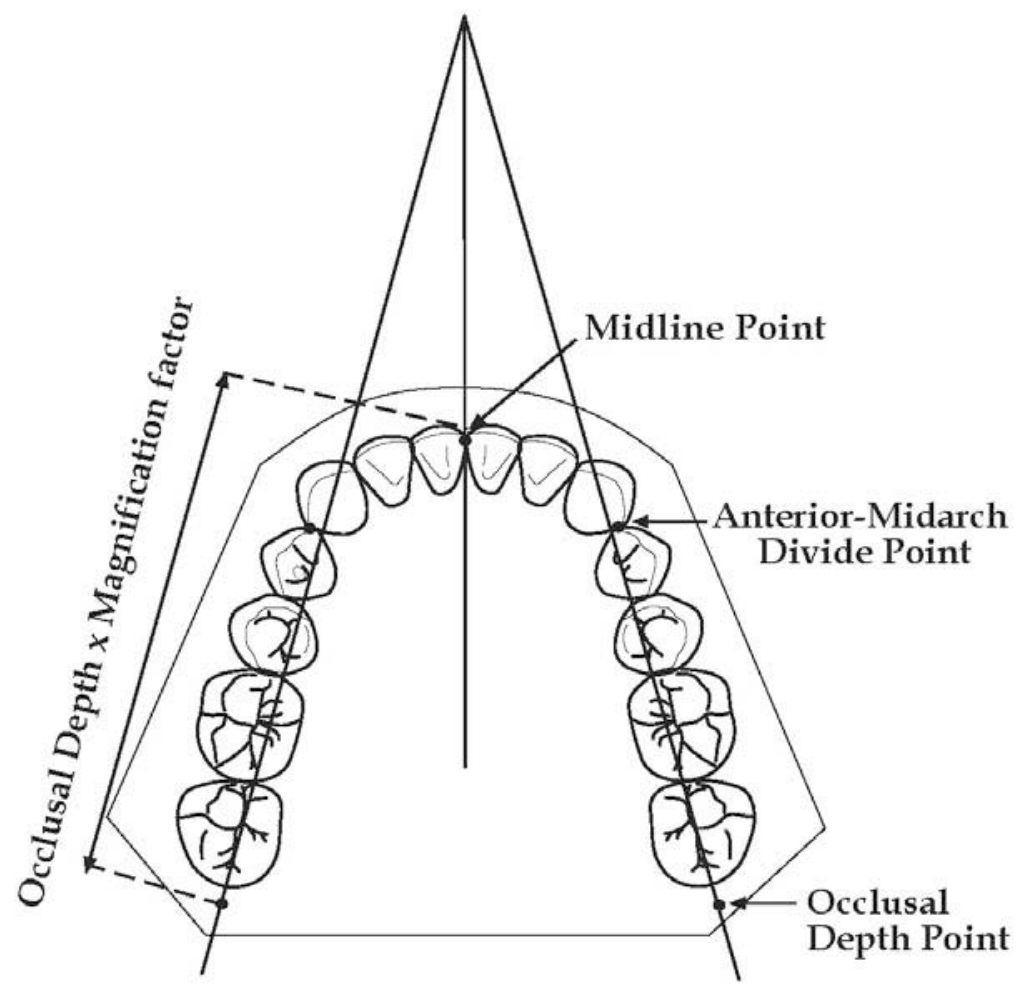

Figure 3-5. Illustration showing midline point and occlusal depth point. The midline point is the intersection of the bisector line and the perpendicular line crossing the incisal edge of the more protrusive central incisor. Occlusal depth points are located distal to the second molars and correspond to the occlusal depth value times the photocopy magnification factor. 


\section{Digitized Landmarks}

The following points were digitized on the casts. Figures 3-2 through 3-9 illustrate these variables.

1. Distal contact point, LL7

2. Mesial contact point, LL7

3. Distal contact point, LL6

4. Mesial contact point, LL6

5. Distal contact point, LL5

6. Mesial contact point, LL5

7. Distal contact point, LL4

8. Mesial contact point, LL4

9. Distal contact point, LL3

10. Mesial contact point, LL3

11. Distal contact point, LL2

12. Mesial contact point, LL2

13. Distal contact point, LL1

14. Mesial contact point, LL1

15. Mesial contact point, LR1

16. Distal contact point, LR1

17. Mesial contact point, LR2

18. Distal contact point, LR2

19. Mesial contact point, LR3

20. Distal contact point, LR3

21. Mesial contact point, LR4

22. Distal contact point, LR4

23. Mesial contact point, LR5

24. Distal contact point, LR5

25. Mesial contact point, LR6

26. Distal contact point, LR6

27. Mesial contact point, LR7

28. Distal contact point, LR7

29. Left occlusal depth point: The posterior limit of the tooth-bearing portion of the mandible.

30. LL6 mesial buccal groove at gum line point.

31. Left divide point: The junction of the left midarch segment and the anterior segment. This point is the distal contact point of LL3 in a stable and wellaligned arch. This point is centered over the alveolus in an irregular arch.

32. LL3 cusp tip point.

33. Midline point: Midline of mandibular arch determined by geometric construction. 


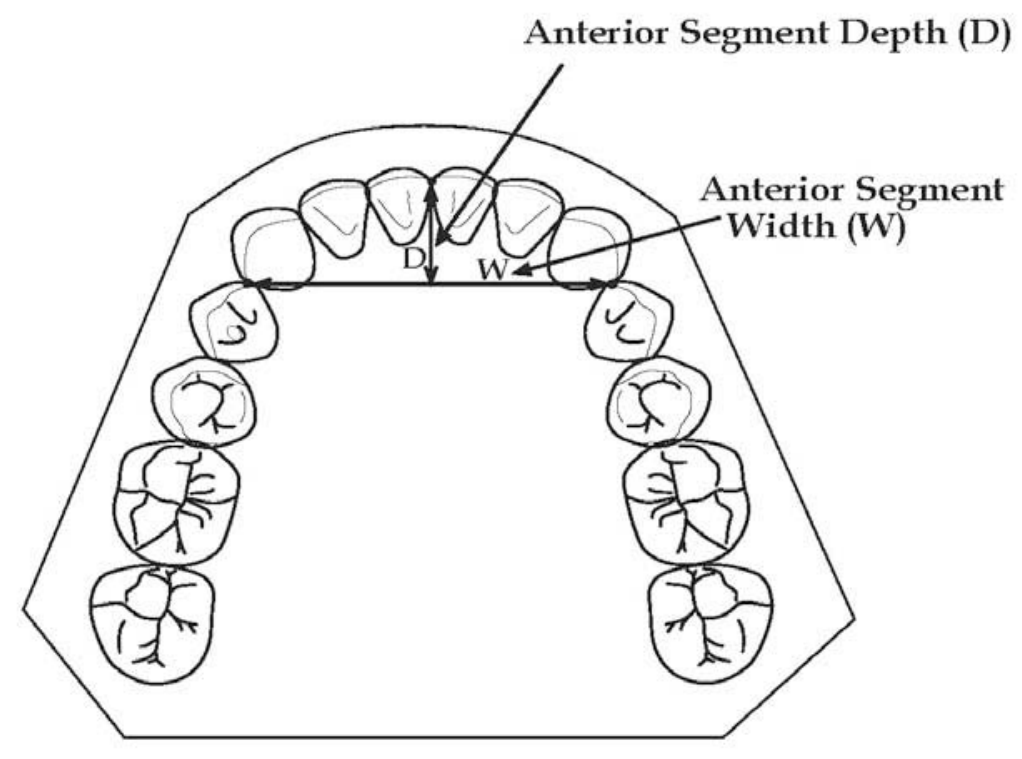

Figure 3-6. Illustration showing anterior segment depth, anterior segment width, and anterior segment ratio.

Anterior segment depth is perpendicular from the midline point to the anterior segment width line. Anterior segment width is the linear distance between the left and right divide points. Anterior segment depth divided by the anterior segment width is the anterior segment ratio. 


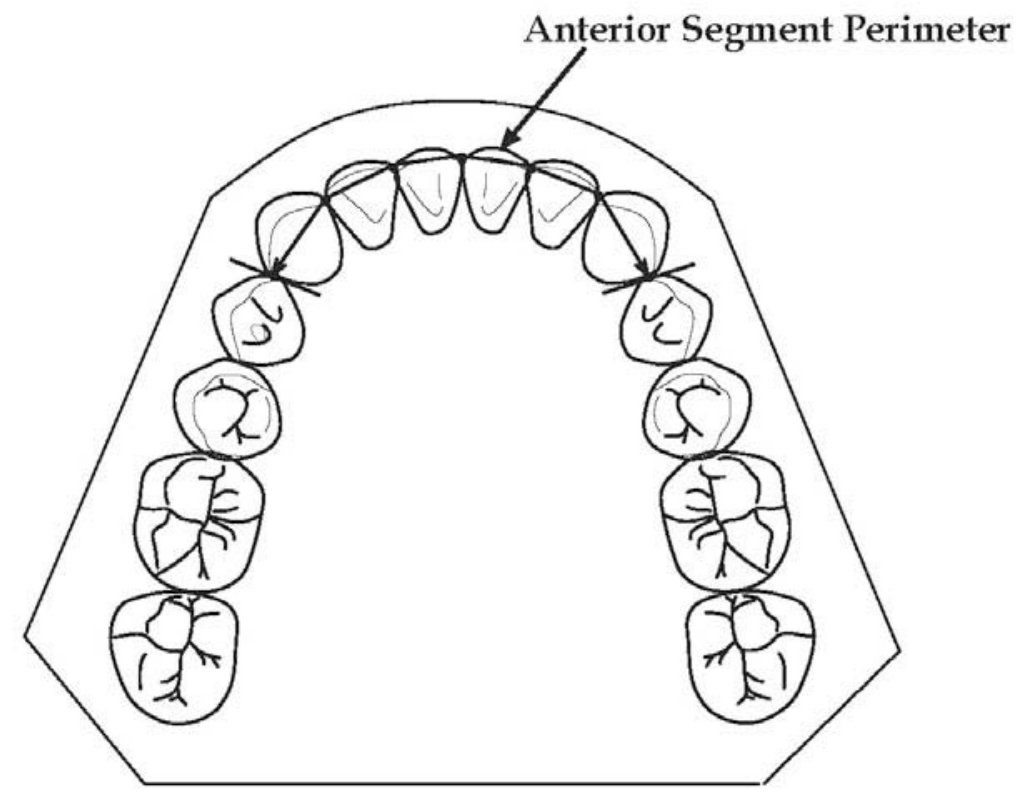

Figure 3-7. Illustration showing anterior segment perimeter. Anterior segment perimeter is the distance available for the placement of six anterior teeth in good alignment between the left and right divide points and the midline. 


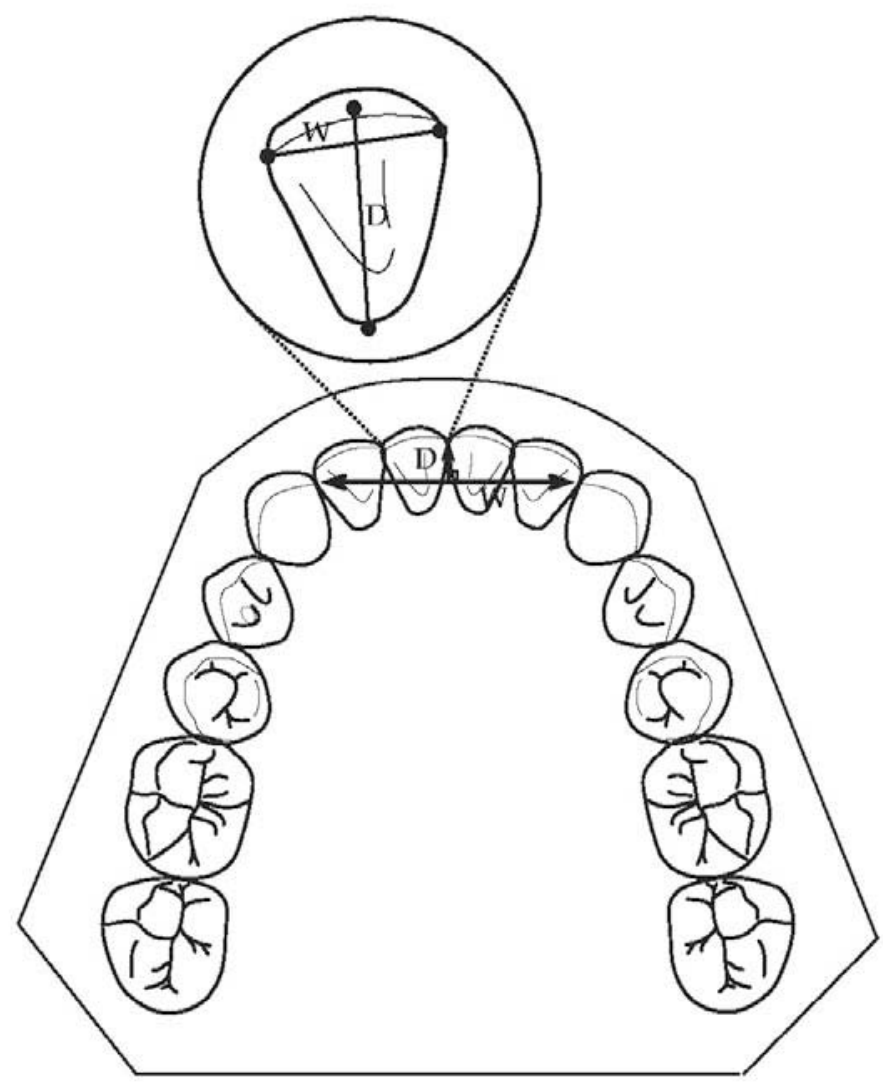

Figure 3-8. Illustration showing incisor aspect ratio and incisor segment ratio. Incisor aspect ratio is the measure of the lower left central incisor mesiodistal width divided by the lingual surface length as viewed from the occlusal. Incisor segment ratio is the measure of the width of the four incisors divided by the distance from the midline point to this line. 


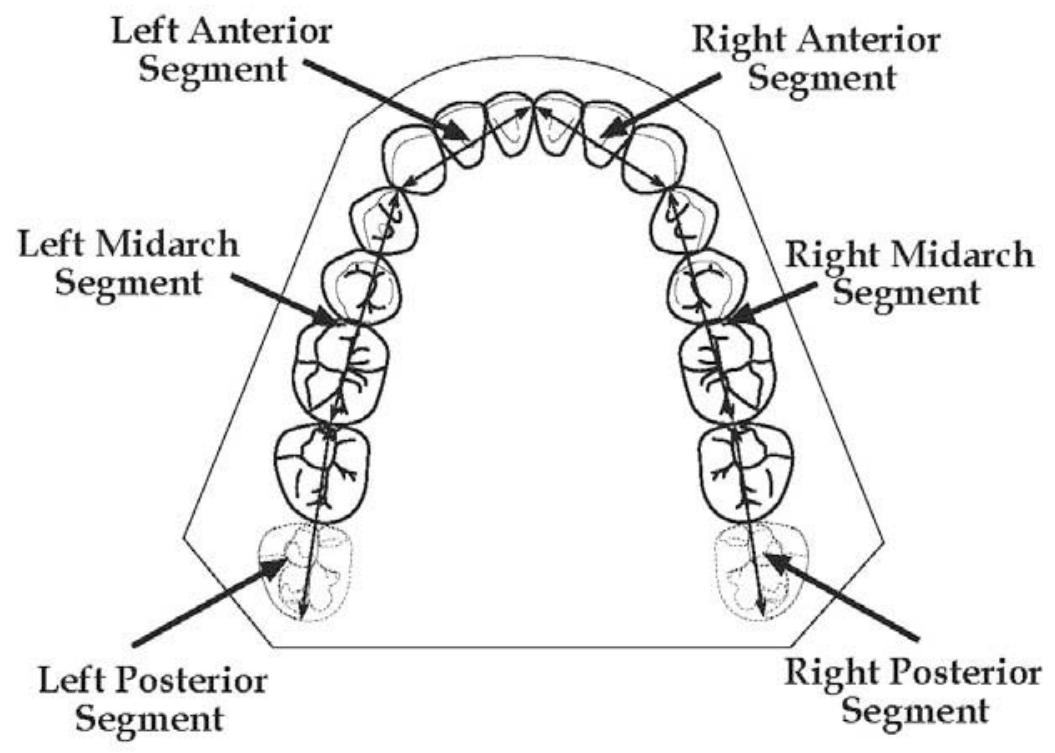

Figure 3-9. Illustration showing mandibular segments.

The left and right anterior segments are measured from the midline point to the anterior divide point. The left and right midarch segments are measured from the anterior divide points to the contact between first and second molars. This is the calculated value of space available for first and second premolars and first molar. The left and right posterior segments are the calculated value of space available for second and third molars. 
34. LR3 cusp tip point.

35. Right divide point: This is the junction of the right midarch segment and the anterior segment. This point is the distal contact point of LR3 in a stable and well-aligned arch. This point is centered over the alveolus in an irregular arch.

36. LR6 mesial buccal groove marked at the gum line.

37. Right occlusal depth point: The posterior limit of the tooth-bearing portion of the mandible.

38. UR6 gum line point: Intersection of the line connecting upper first molar mesial buccal cusp tips and gum line UR6.

39. UR3 cusp tip.

40. UL3 cusp tip.

41. UL6 gum line point: Intersection of the line connecting upper first molar mesial buccal cusp tips and gum line of UL6.

42. Intersection of the line connecting upper first molar mesial buccal cusp tips and midline of the palate.

43. H point: A point on the midline of the mandibular occlusal plane.

44. Lingual gum line of LL1.

\section{Cast Analysis}

The Tweed Foundation Research Committee performed a cast analysis using the landmarks just described, and we selected from among those variables for the present study. A total of 19 variables were analyzed, (1) anterior discrepancy, (2) midarch discrepancy, (3) Curve of Spee, (4) molar correction, (5) anterior segment ratio, (6) incisor segment ratio, (7) incisor aspect ratio, (8) anterior segment perimeter, (9) maxillary intermolar width, (10) mandibular intermolar width, (11) maxillary intercanine width, (12) mandibular intercanine width, (13) incisor segment depth, (14) anterior segment depth, (15) incisor depth, (16) anterior segment width, (17) mandibular Incisor Irregularity index (Little 1975), (18) overbite, (19) overjet.

The following listing provides abbreviations and definitions of the cast variables used in the study:

1. ADisc, Anterior Discrepancy: This represents the lower anterior tooth size, arch length discrepancy in millimeters (based on the 4 incisors and 2 cuspids).

2. MADisc, Midarch Discrepancy: This represents the lower midarch tooth size, arch length discrepancy in millimeters (based on the 4 premolars and 2 first molars).

3. COS, Curve of Spee: The perpendicular distance (in millimeters) from a 
straight edge across the occlusal plane to the deepest portion of the Curve of Spee (Figure 3-10).

4. MC, Molar Correction: With casts held in occlusion, the distance is recorded from mesiobuccal cusp tip of U6 to $1 \mathrm{~mm}$ distal of the mesiobuccal groove of L6 (Figure 3-2).

5. ASR, Anterior Segment Ratio: The ratio of the Anterior Segment Depth divided by the Anterior Segment Width (Figure 3-6).

6. ISR, Incisor Segment Ratio: Similar to the ASR, but this dimension contains only the four mandibular incisors. Incisor segment ratio is the measure of the width of the four incisors divided by the distance from the midline point to this line (Figure 3-8). A lesser value indicates a more curved arc formed by the incisal edges of the four incisors as viewed occlusally.

7. IAR, Incisor Aspect Ratio: The ratio of the depth of the lingual aspect of the mandibular left central incisor divided by the width as viewed occlusally (Figure 3-8). A greater value indicates a more upright incisor position.

8. ASP, Anterior Segment Perimeter: The calculated distance available for the placement of six anterior teeth in alignment between the right and left divide points and the midline point (Figure 3-7).

9. UMW, Maxillary Intermolar Width: The linear distance (in millimeters) from the mesiobuccal cusp tip of the upper (maxillary) right first molar to the mesiobuccal cusp tip of the upper (maxillary) left first molar.

10. LMW, Mandibular Intermolar Width: The linear distance (in millimeters) from the mesiobuccal cusp tip of the lower (mandibular) right first molar to the mesiobuccal cusp tip of the lower (mandibular) left first molar.

11. UCW, Maxillary Intercanine Width: The linear distance (in millimeters) from the upper (maxillary) right canine cusp tip to the upper (maxillary) left canine cusp tip.

12. LCW, Mandibular Intercanine Width: The linear distance (in millimeters) from the lower (mandibular) right canine cusp tip to the lower (mandibular) left canine cusp tip.

13. ISD, Incisor Segment Depth: The perpendicular distance (in millimeters) from the midline point (Figure 3-5) to the Incisor Segment Width line (Figure 3-8; the line that delineates the width of the four incisors). 


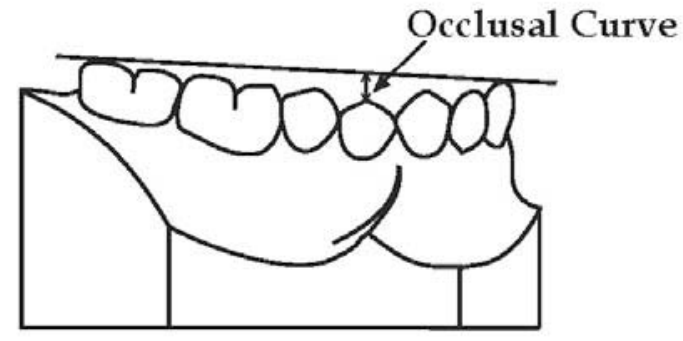

Figure 3-10. Illustration showing occlusal curve.

The vertical distance is measured from a plane across the occlusal plane to the deepest portion of the Curve of Spee. 
14. ASD, Anterior Segment Depth: The perpendicular distance (in millimeters) from the midline point to the Anterior Segment Width line (Figure 3-6).

15. ID, Incisor Depth: The linear distance (in millimeters) from the midline point to $\mathrm{H}$ point.

16 ASW, Anterior Segment Width: The linear distance (in millimeters) from between the left and right divide points (Figure 3-6).

17. II, Mandibular Incisor Irregularity Index (Little 1975): The sum of the distance between each of the five contact points of the lower anterior teeth (Figure 3-11).

18. OB, Overbite: The greatest linear distance (in millimeters) from the incisal edge of a maxillary incisor to the incisal edge of a mandibular incisor (Figure 3-12). A negative value reflects an anterior open bite situation.

19. OJ, Overjet: The greatest linear distance (in millimeters) from the incisal edge of a maxillary incisor to the labial surface of the corresponding mandibular incisor (Figure 3-12). A negative value reflects an anterior crossbite situation.

\section{Cephalometric Analysis}

The Tweed Foundation Research Committee also performed a cephalometric analysis, and we selected from among those variables for the present study. The following 13 variables were analyzed here, (1) Frankfort mandibular incisor angle (FMIA), (2) Frankfort mandibular angle (FMA), (3) incisor mandibular plane angle (IMPA), (4) Sella-Nasion to A Point angle (SNA), (5) Sella-Nasion to B Point angle (SNB), (6) A Point to Nasion to B Point angle (ANB), (7) AOBO (Wits appraisal), (8) Downs' occlusal plane (DOP), (9) Z angle, (10) upper lip (UL), (11) total chin (TC), (12) anterior facial height (AFH), and (13) posterior facial height $(\mathrm{PFH})$.

\section{Cephalometric Landmarks}

1. A Point, (Subspinale): The most posterior point on the exterior ventral curve of the maxilla between the anterior nasal spine and Supradentale.

2. B, Point B (Supramentale): The most posterior point on the bony curvature of the mandible between Infradentale and Pogonion. 


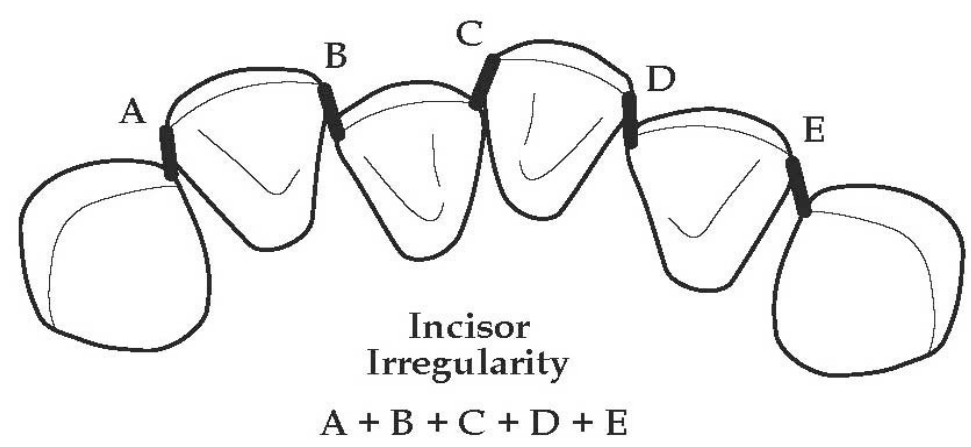

Figure 3-11. Illustration of Incisor Irregularity as measured in this study. The sum of the five distances between the dental contact points is Incisor Irregularity. 

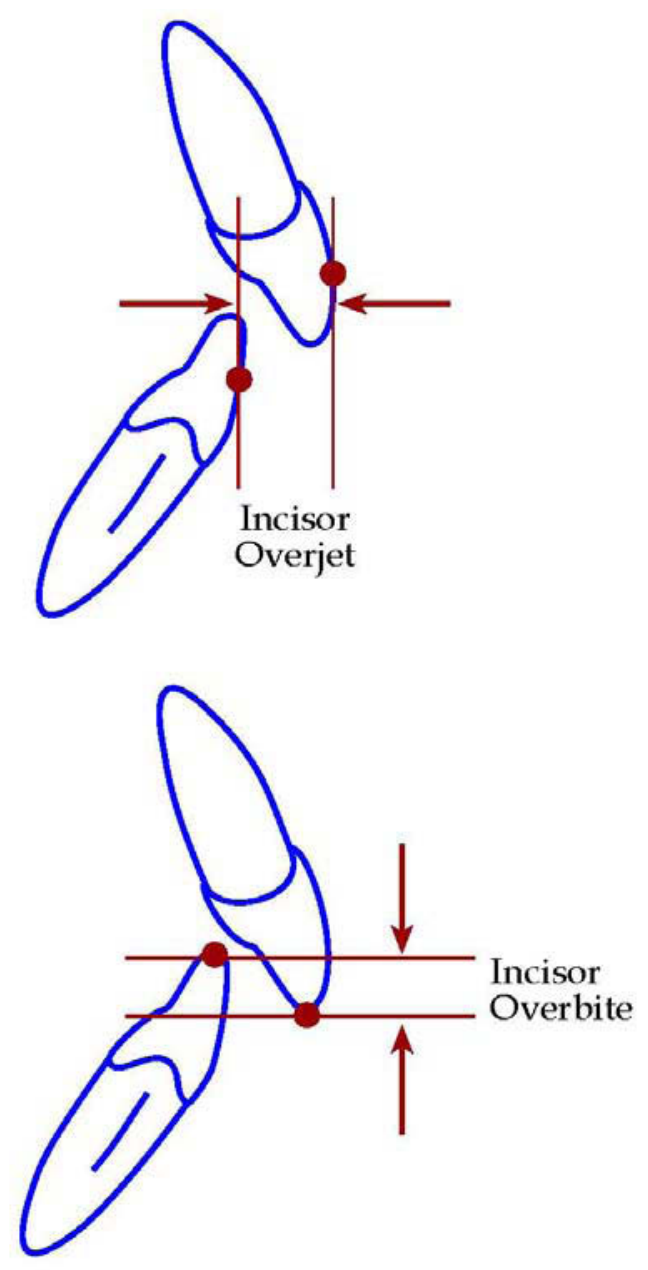

Figure 3-12. Illustration of the incisor overjet and incisor overbite measurements. 
3. Go, Gonion (anatomic): The most posterior-inferior point along the gonial process of the mandible.

4. Is, Incision superius: The incisal tip of the more anterior maxillary central incisor.

5. Ii, Incision inferius: The incisal tip of the more anterior mandibular central incisor.

6. Ls, Labrale superius: The most anterior point on the vermilion border of the upper lip.

7. Li, Labrale inferius: The most anterior point on the vermilion border of the lower lip.

8. LIA, Apex of mandibular central incisor: The apical end of the same mandibular central incisor used to locate Ii.

9. L6C, L6 cusp: The mesial cusp tip of the mandibular first molar.

10. Me, Menton: The most inferior point on the symphysis of the mandible.

11. Na, Nasion: The anterior point at the intersection between the nasal and frontal bones.

12. Or, Orbitale: The most inferior point on the lower margin of the bony orbit.

13. Pg, Pogonion: The most anterior point on the anterior contour of the bony chin below B Point and above Gnathion.

14. Se, Sella turcica: The center of the hypophyseal fossa, determined by inspection.

15. U1A: the apical end of the maxillary central incisor.

\section{Computed Measurements}

All landmarks are located with the image of the subject's head oriented with Frankfort Horizontal parallel to the floor. The following listing provides definitions of the cephalometric variables used in the study: 
1. ANB, A Point to Nasion to B Point angle: The inferior angle formed at the junction of the Nasion-A Point line and the Nasion-B Point line (Figure 3-13).

2. AOBO: The linear distance between two points along Downs' occlusal plane (Downs 1948) obtained from the intersection of a perpendicular line from point $A$ and from point $B$ to the occlusal plane (Figure 3-14). This variable is also referred to as the Wits appraisal (Jacobson 1975, 1976). In the present study, Downs' occlusal plane was used to calculate AOBO, even though Jacobson promoted the use of the functional occlusal plane.

3. DOP, Downs' Occlusal Plane: The line that bisects the maxillary and mandibular incisal overbite and the most anterior occlusal contact of the maxillary and mandibular first molars (Downs 1948).

4. FMA, Frankfort Mandibular Plane Angle: The anterior-inferior angle formed at the junction of the Frankfort Horizontal plane and the mandibular plane (Figure 3-15). Mandibular plane is defined by two landmarks, namely anatomic Gonion and Menton.

5. FMIA, Frankfort Mandibular Incisor Angle: The posterior-inferior angle formed between the Frankfort Horizontal plane and the long axis of the mandibular central incisor (Figure 3-16).

6. IMPA, Incisor Mandibular Plane Angle: The posterior-superior angle formed at the junction of the mandibular plane and the long axis of the mandibular central incisor. The long axis of the mandibular incisor is defined by Ii and L1 apex (Figure 3-17).

7. SNA, Sella-Nasion A Point angle: The posterior inferior angle formed at the junction of the Sella-Nasion plane and the Nasion-A Point plane (Figure 3-18).

8. SNB, Sella-Nasion B Point angle: The posterior inferior angle formed at the junction of the Sella-Nasion plane and the Nasion-B Point plane (Figure 3-19).

9. $\mathrm{Z}$ angle: The inferior angle formed at the intersection of the Frankfort Horizontal line and the profile line (Merrifield 1966), where the profile line is defined by Pronasale and either Li or Ls depending on which lip in more protrusive (Figure 3-20).

10. UL, Upper Lip: The millimeter measurement of the horizontal thickness of the upper lip, recorded from Prosthion to the most anterior point on the vermilion border of the upper lip (Merrifield 1966; Figure 3-21). 


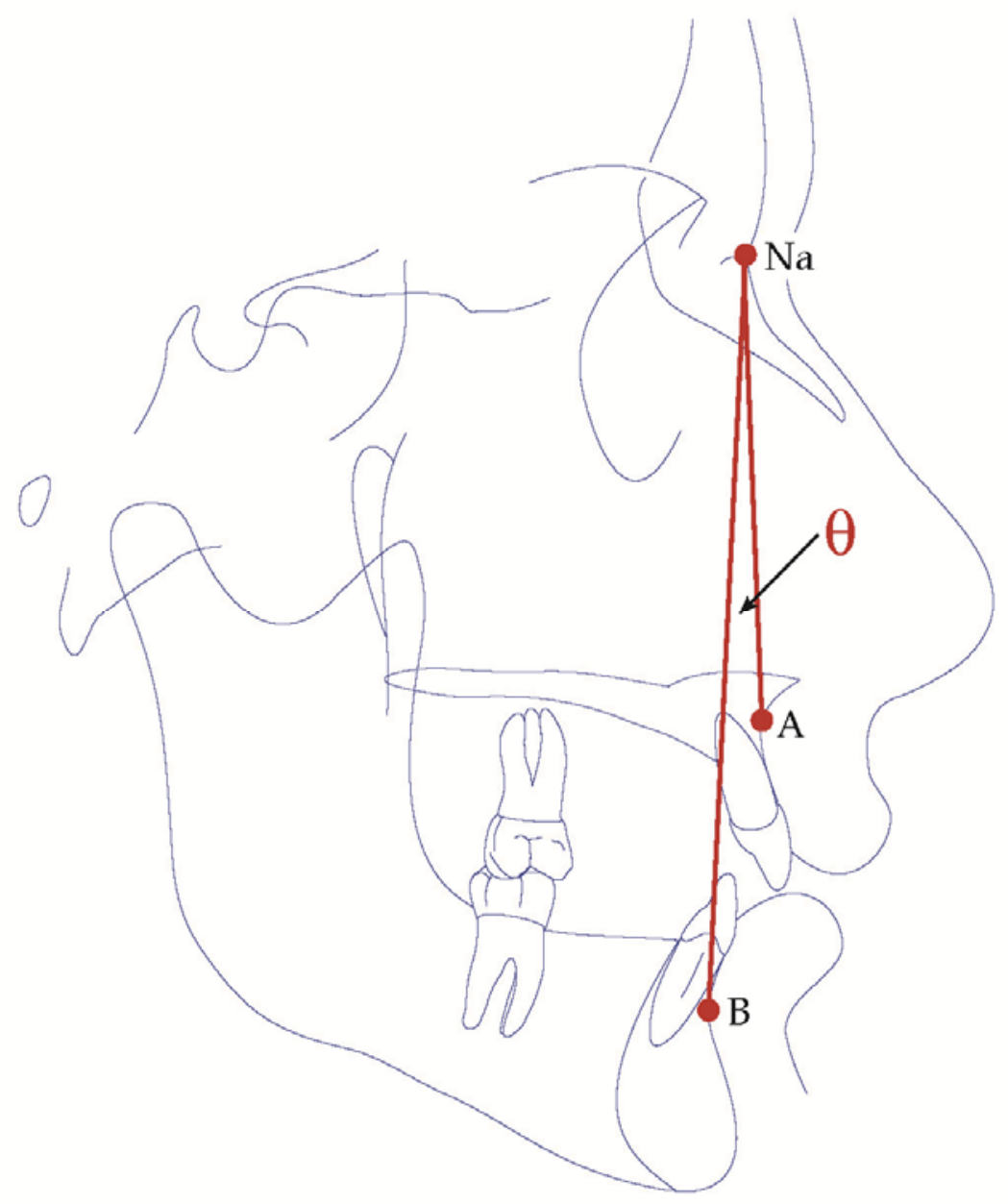

Figure 3-13. Depiction of the angle ANB (denoted as $\theta$ ) as viewed on a lateral cephalogram. 


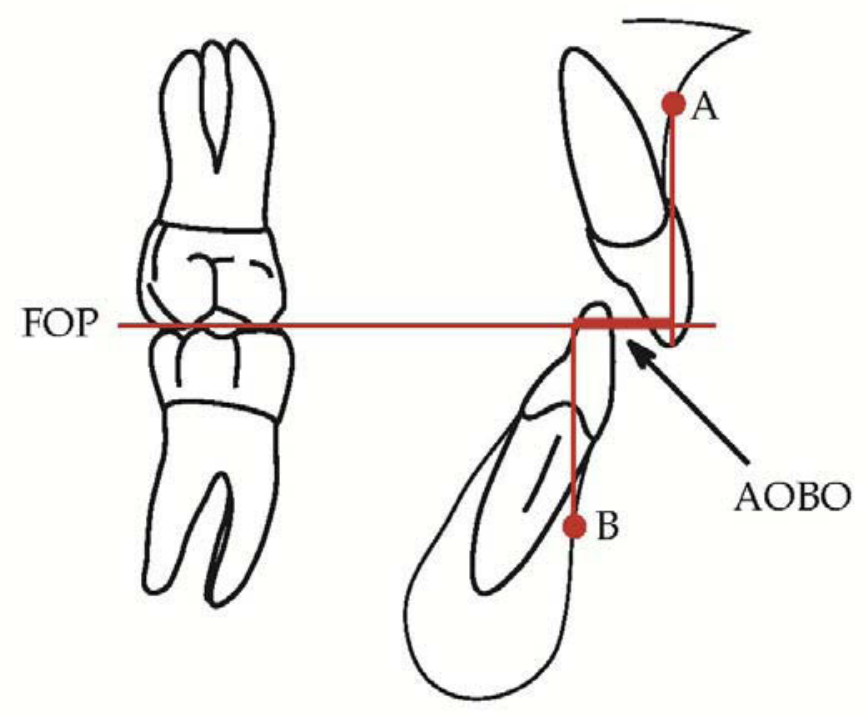

Figure 3-14. Depiction of the horizontal measurement of AOBO from a lateral cephalogram.

FOP is the functional occlusal plane. 


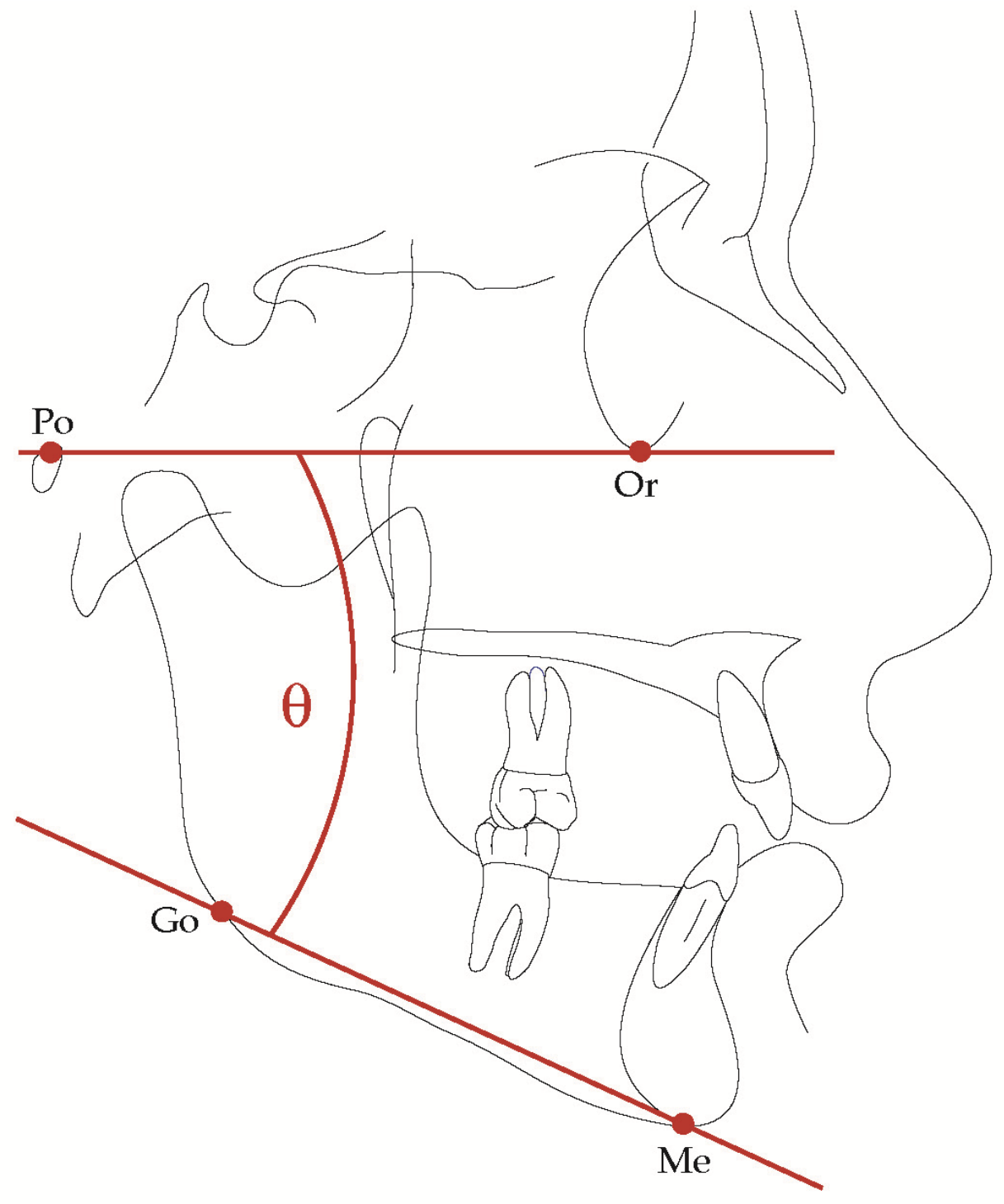

Figure 3-15. Illustration of the measurement of FMA (denoted as $\theta$ ) from a lateral cephalogram. 


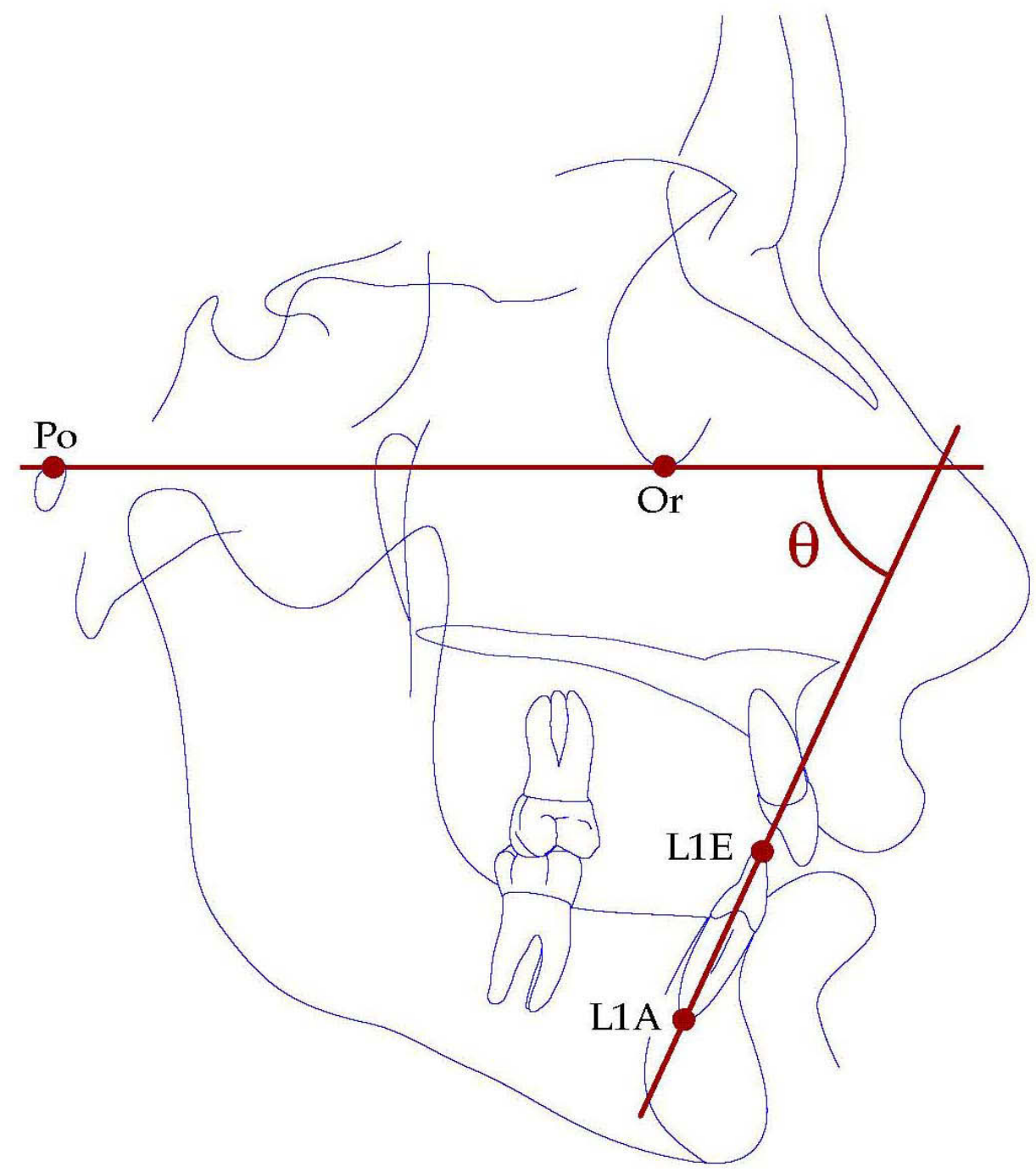

Figure 3-16. Illustration of the measurement of FMIA (denoted as $\theta$ ) from a lateral cephalogram. 


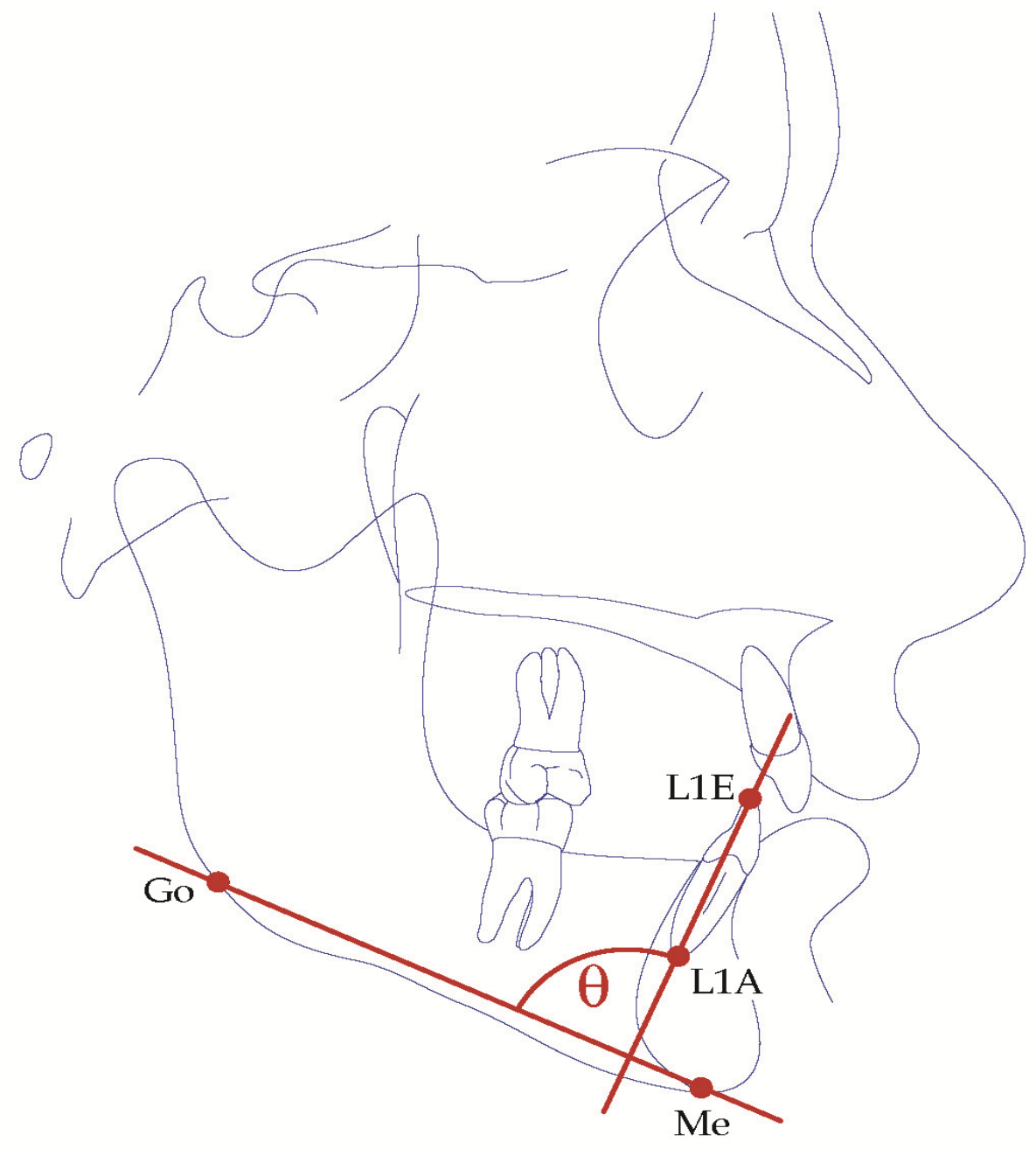

Figure 3-17. Illustration of the measurement of IMPA (denoted as $\theta$ ) from a lateral cephalogram. 


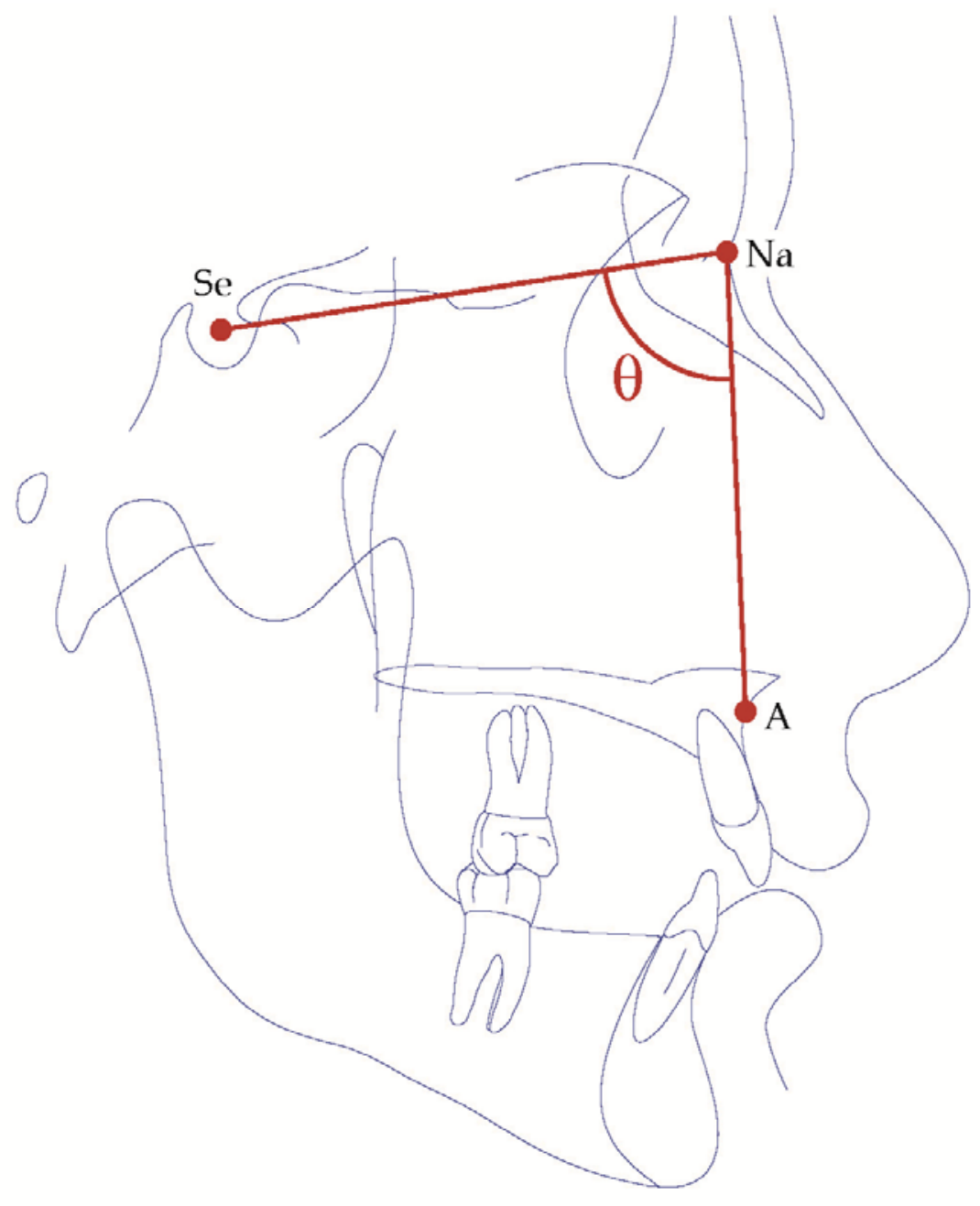

Figure 3-18. Illustration of the measurement of the angle SNA (denoted as $\theta$ ) from a lateral cephalogram. 


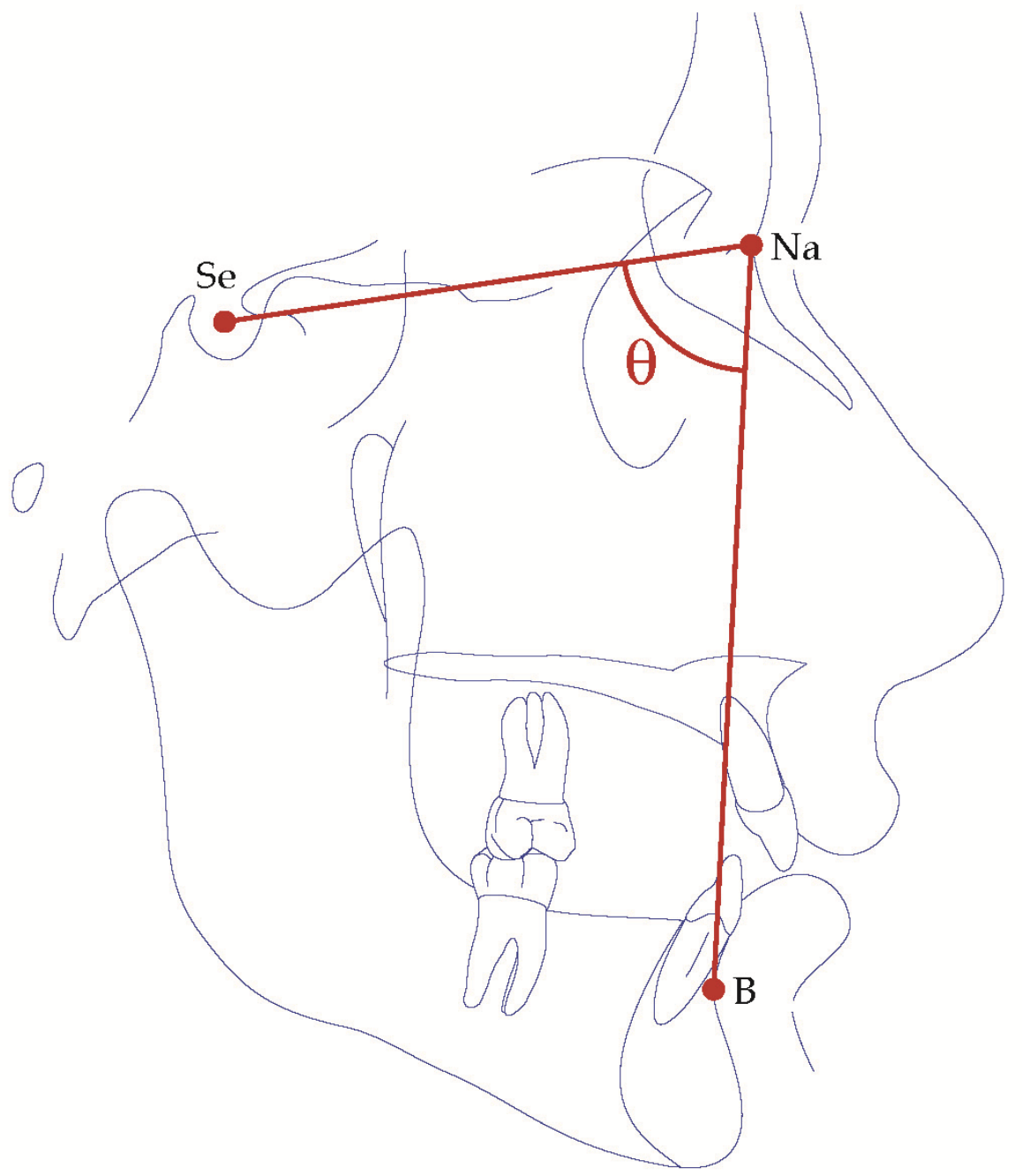

Figure 3-19. Illustration of the angle SNB (denoted as $\theta$ ) on a lateral cephalogram. 


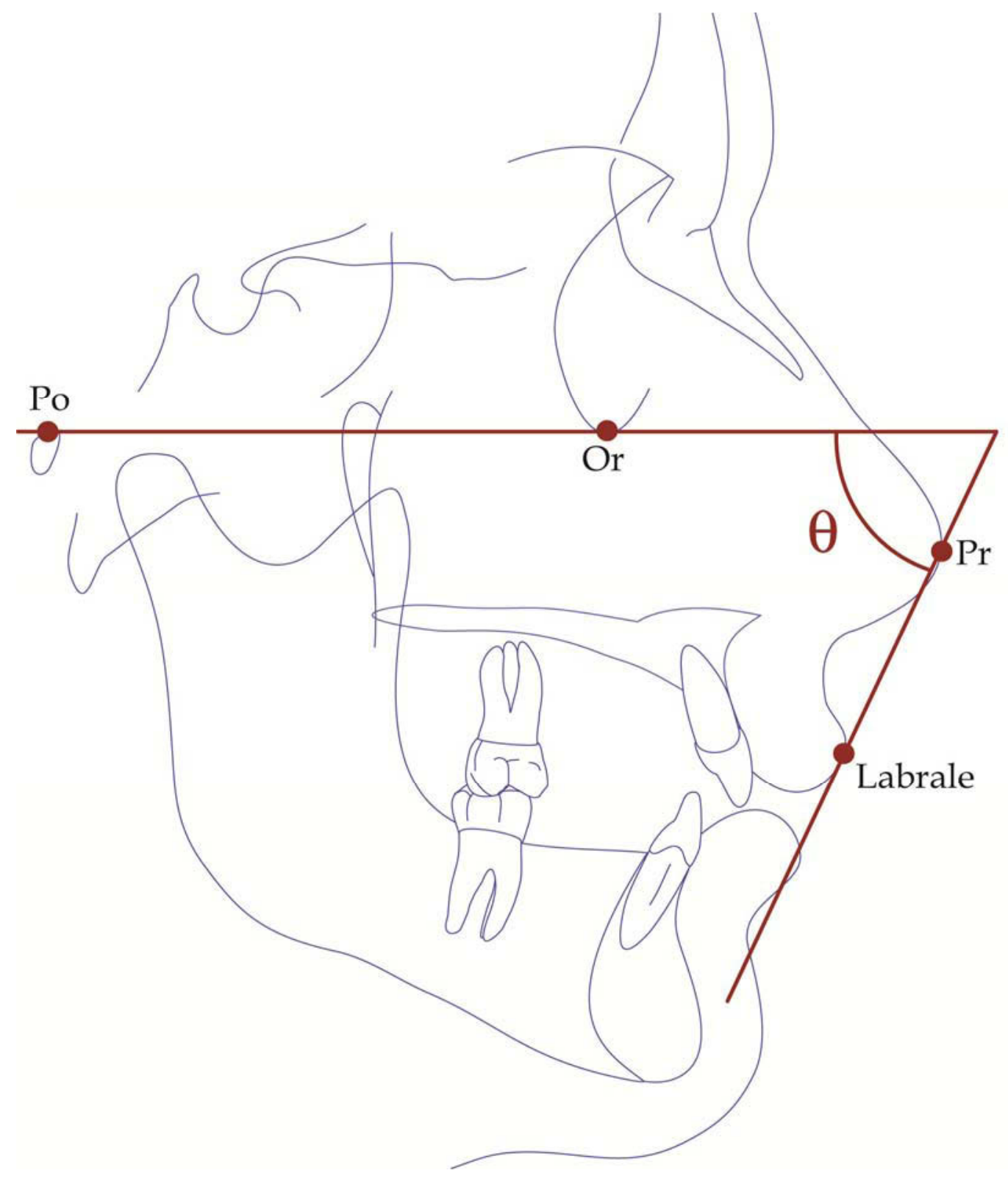

Figure 3-20. Illustration of the $\mathrm{Z}$ angle (denoted as $\theta$ ) on a lateral cephalogram. 


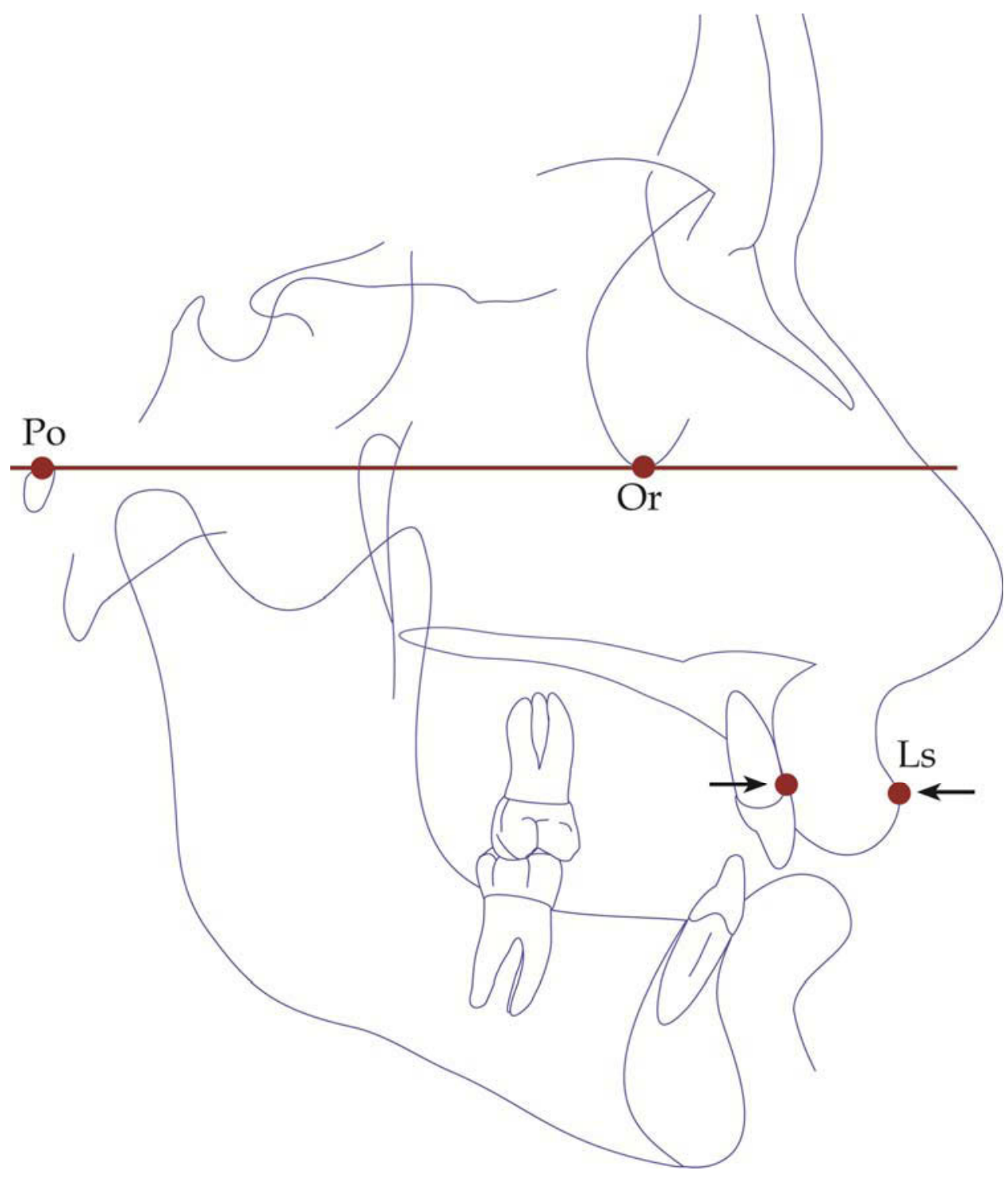

Figure 3-21. Illustration of the upper lip measurement on a lateral cephalogram. 
11. TC, Total Chin: The millimeter measurement including the bony chin lying anterior to the NB line and measured to soft tissue Pogonion (Merrifield 1966; Figure 3-22). The total chin thickness should be equal to or slightly greater than the upper lip thickness.

12. AFH, Anterior Facial Height: The linear measurement from palatal plane to Menton, measured perpendicular to palatal plane (Gebeck and Merrifield 1995; Figure 3-23).

13. PFH, Posterior Facial Height: The linear measurement from Articulare, along the line tangent to the posterior border of the mandible (Gonion to Menton line), to the intersection with the mandibular plane (Gebeck and Merrifield 1995; Figure 3-24).

\section{Statistical Analysis}

Data were collated into an Excel spreadsheet (Microsoft ${ }^{\circledR}$ Corporation, Redmond, WA) then transferred to the JMP 8.0.1 statistical package (SAS Institute Inc., Cary, NC). Exploratory data analysis (Tukey 1977) was performed, searching for outliers; those due to technical errors were corrected. Conventional descriptive statistics (e.g., Sokal and Rohlf 1995) were calculated; these (and their abbreviations) are sample size (n, taken as counts of individuals, not sides), the arithmetic mean $(\bar{x})$, the standard deviation $(s d)$, the sample variance $\left(s^{2}\right)$, the standard error of the mean (sem, calculated as $\mathrm{sd} / \sqrt{ } \mathrm{n}$ ), upper and lower $95 \%$ confidence limits of the distribution $\left(\mathrm{L}_{1}, \mathrm{~L}_{2}\right)$, skewness (g1), kurtosis (g2), coefficient of variation (cv), number of cases missing, maximum value, median value (50th percentile), and minimum value. The conventional alpha level of 0.05 was used throughout, and all of the tests were two-tail. No correction was made for multiple comparisons. Salient results of the analysis were graphed using JMP 8.

Skeletodental differences were calculated between the 3 examinations, namely (A) in-treatment changes (calculated as posttreatment-minuspretreatment) and (B) posttreatment changes (calculated as recall-minusposttreatment).

One sample t-tests (two tail) were used to evaluate changes in variables across time as a function of the sample variability. Repeated measures analysis of variance (Winer et al. 1991) was used to determine if the changes between the examinations differed significantly from one another depending on sex and/or type of retention. 


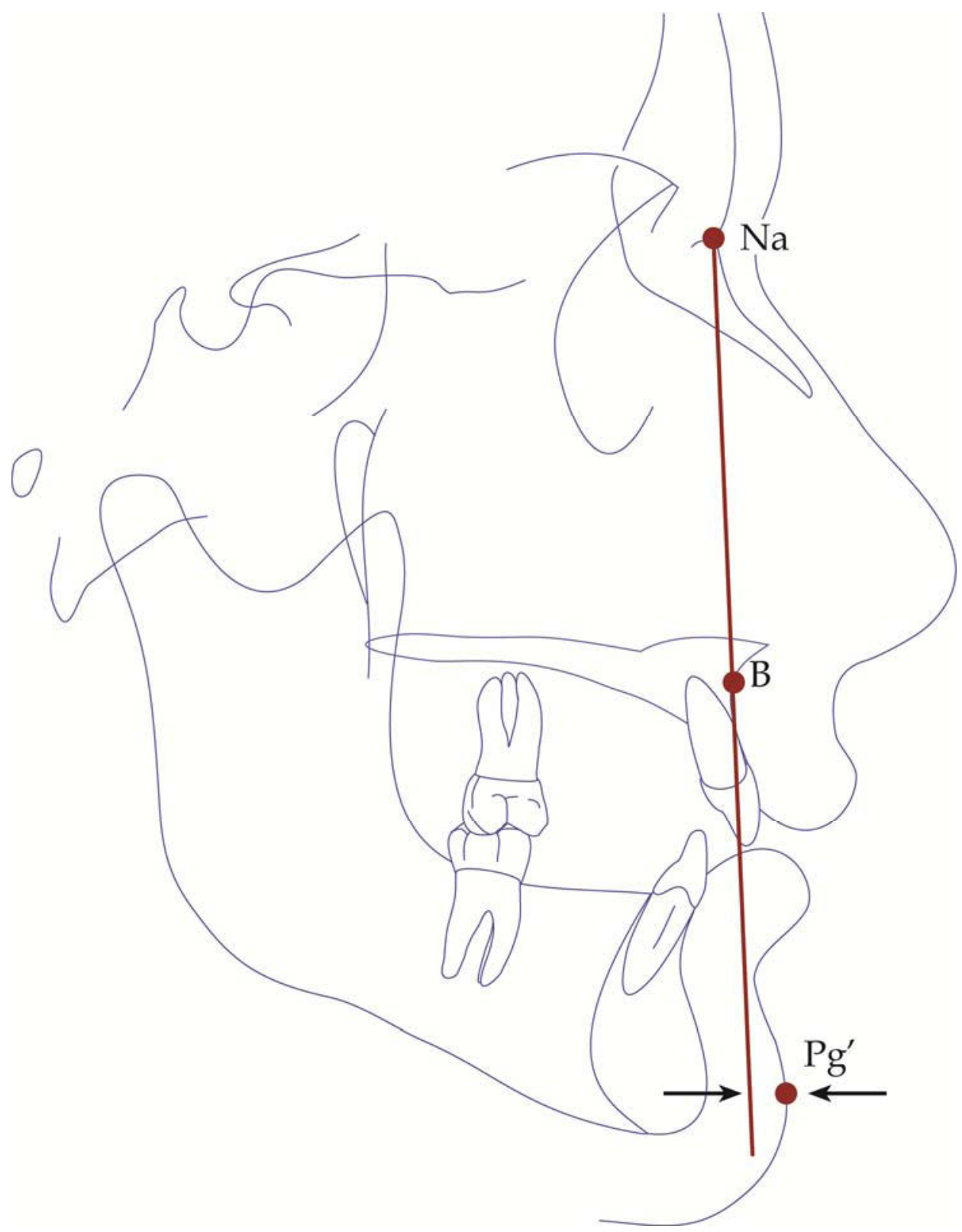

Figure 3-22. Illustration of the total chin measurement on a lateral cephalogram. 


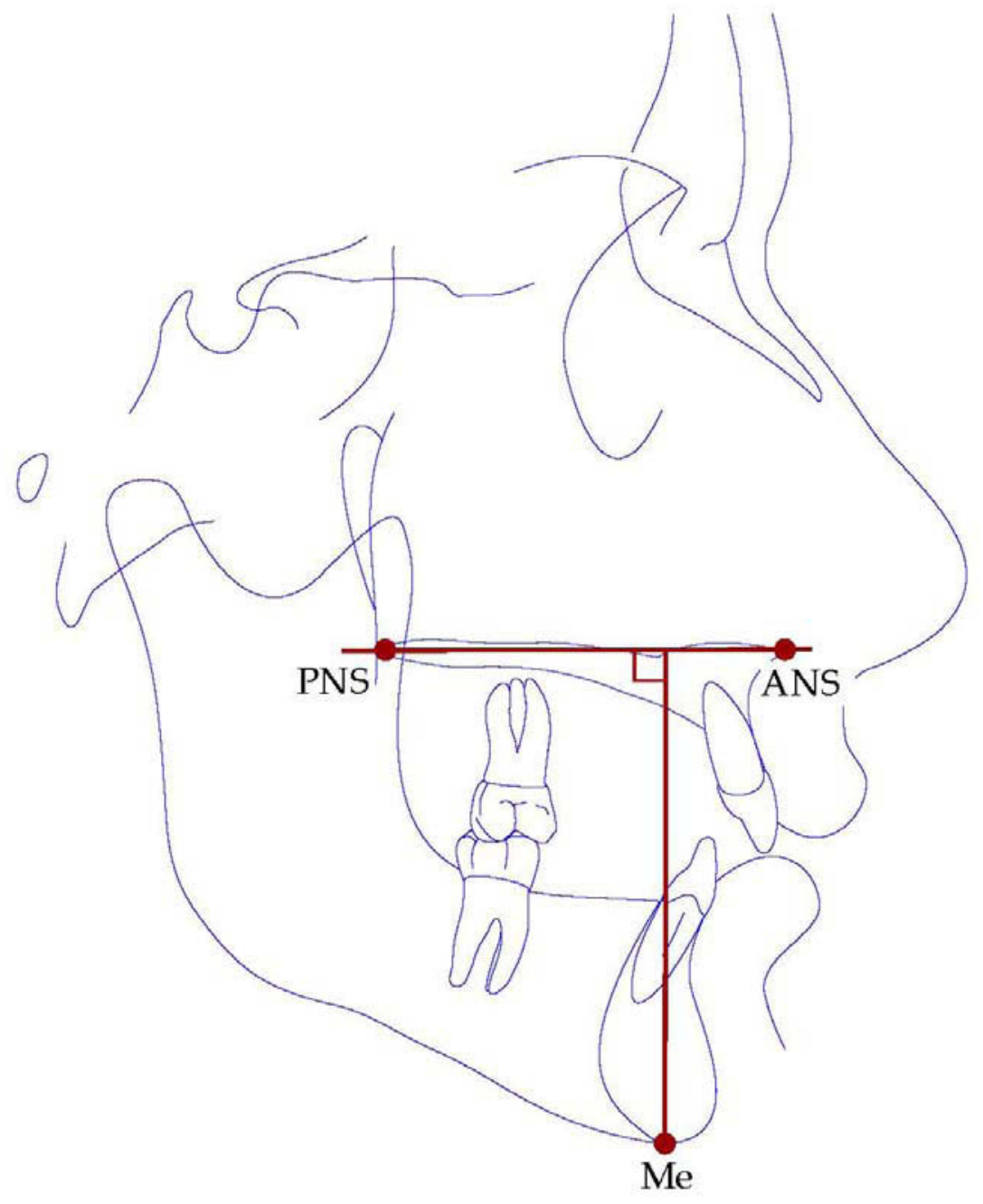

Figure 3-23. Illustration of the anterior facial height measurement on a lateral cephalogram. 


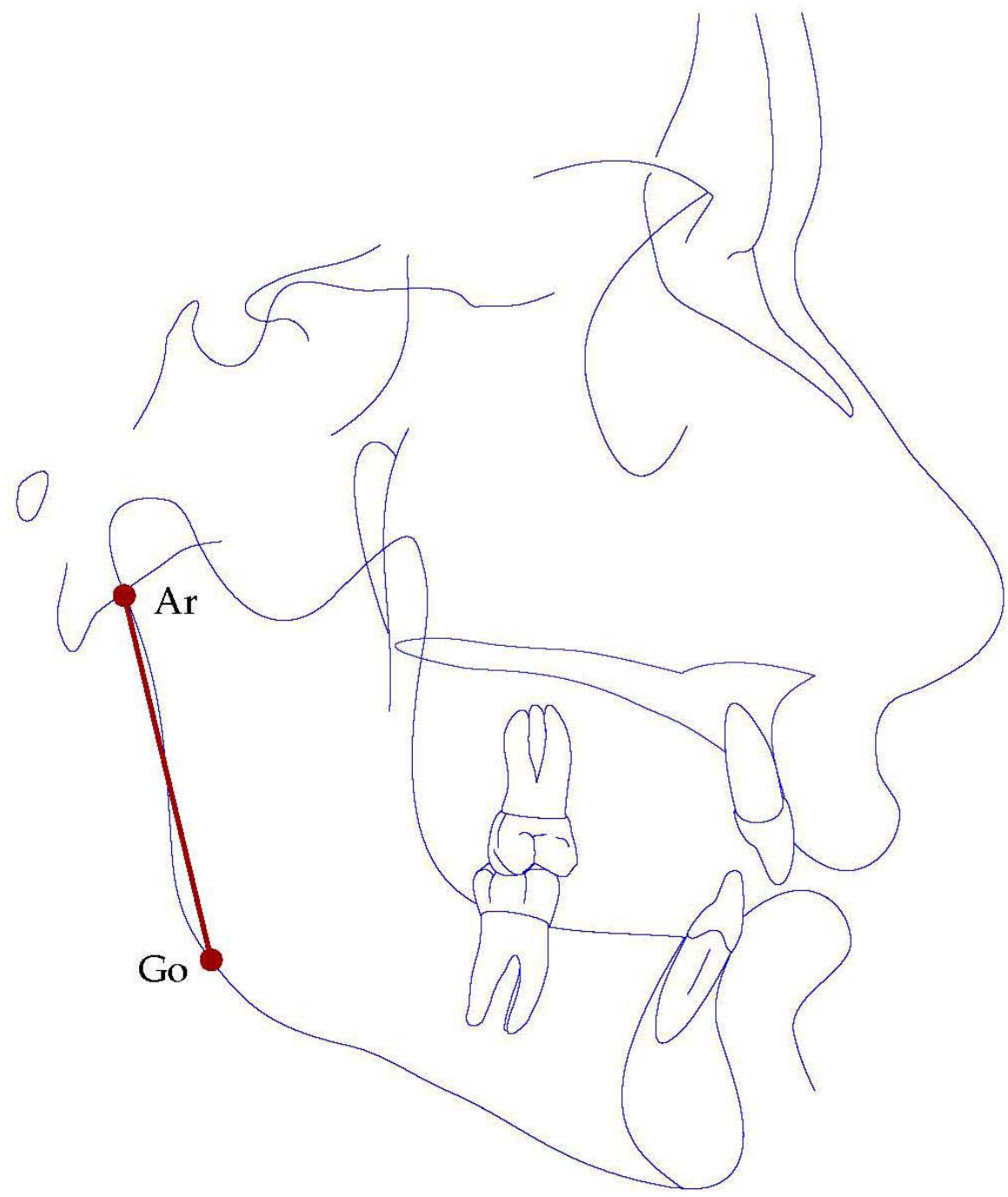

Figure 3-24. Illustration of the posterior facial height measurement on a lateral cephalogram. 


\section{Sample Demographics}

Boley (2007, p 13) suggested that, "Perhaps the best sample in the literature is one in which every patient was treated in the same manner." The orthodontic records obtained for use in this study were submitted by standard Edgewise practitioners from across the North American continent. With the submission of cases by multiple practitioners, the influence of individual practitioner biases and treatment prejudices is diminished. It is the intent of the research to be based on a representative sample of patients. Practitioners were told not to cherry-pick their cases when submitting records, and our study, like all studies, has to rely on the honesty and integrity of those submitting the records. It is thought that practitioners could not be too discriminatory, because in reality, there is not a great availability of records at 10 or more years out of treatment. As in all data collection of this type, you cannot force people to come in for recall records.

These types of records are more a matter of convenience, opportunity, and availability rather than premeditated choice. It seems likely that if a patient has considerable relapse, he or she probably recognizes his own condition, and might be less likely to return for recall records because of the potential for embarrassment or anticipated chastising for lack of retainer wear (i.e., the University of Washington studies where residents had to call and attempt to recall patients). This factor could add bias into any sample of this type.

Also, as evidenced in the recall records of many studies of this type, the availability of males is less common than females. This supports the contention that males are less likely to be compliant following treatment. There is the supposition that males are less diligent about retainer wear than females.

\section{Edgewise Mechanics}

The Edgewise appliance is characterized by not having any tips, slants, or variations in thickness of the bracket or slot. All slots are $0.022^{\prime \prime} \times 0.028^{\prime \prime}$ in dimension and are placed so that they are at right angles to the long axes of the teeth. The technique uses both round and rectangular archwires. Elastics and directionally oriented headgear (high-pull and straight-pull to J-hooks) are routinely used auxiliaries.

The following description of Edgewise treatment mechanics is as described by Merrifield (1985) and Sandusky and Gramling (1988). The new Tweed force system has undergone an evolution as defined by Vaden, Dale, and Klontz (2000). However, considering that the present patients were treated by 
the "older" Tweed force system, an explanation of those treatment mechanics follows. The technique can be divided into four phases: (1) denture preparation; (2) denture correction; (3) denture completion; and 4) denture recovery. Denture preparation includes all the initial steps required to ready a malocclusion for correction, namely (1) leveling, (2) individual tooth alignment, (3) canine retraction, and (4) initial preparation of the terminal molars for anchorage. Treatment is begun with small diameter round wires that incorporate tip-back bends for the terminal molars. These are to maintain the maxillary distal-axial inclination and to level the mandibular Curve of Spee. J-hook headgear is fitted to the canine brackets in both arches for canine retention.

The objectives of denture correction are (1) retraction and uprighting of the incisors to their planned positions, (2) completion of space closure, and (3) achievement of posterior and midarch axial inclination that will permit proper functional coordination with the maxillary teeth.

Rectangular archwires with appropriate first, second, and third order bends are used during this treatment period. Closed vertical loops distal to the lateral incisors are activated by ligating posterior loop stops, and further activation is achieved by applying high-pull J-hook headgear to anterior spurs soldered gingivally between the central and lateral incisors.

The headgear is adjusted to deliver its force at an angle of approximately 40 degrees above the occlusal plane. This orientation provides a vertical component to overcome the intrusive effect of the second order bends placed at the terminal molars and, at the same time, there is a distal component that produces incisor uprighting. By supporting the archwire with the high-pull headgear, the two terminal molars will assume an anchorage position with approximately 10 degrees of distal tip for maxillary first molars and 15 degrees of tip for mandibular second molars.

When all the spaces are closed and the incisors are retracted, the spaceclosing archwire is replaced by a $0.0215^{\prime \prime} \times 0.028^{\prime \prime}$ continuous archwire. This wire has essentially the same first and third order bends with modified second order bends. With the continuation of the high-pull headgear force, the second order bends at the terminal molars are maintained while a distal tip of approximately 10 degrees is added at the first molar. It is necessary to place a compensating bend mesial to the loop stop to allow for distal inclination of the second molar. Once the molars have tipped distally, a 10-degree tip is added to the archwire at the second premolar region and appropriate compensating bends are added mesial to the first molar. 
When this is completed, the first and second molars will be inclined distally and out of occlusion (except the mesial cusp of the first molars). For patients with Class II malocclusions, an additional system of forces must be used to complete the correction.

The Class II force system uses the previously described mandibular archwire, which is clinched and ligated for maximum stability. Placing a closed bulbous loop flush with the mesial end of the molar tube modifies the maxillary archwire. Sliding jigs are then employed against the first molar with a Class II elastic force as the bulbous loops are opened. Anterior vertical box elastics are needed to complete the system.

After over-correction of the Class II relationship, a new maxillary archwire may be needed to close spaces opened by the jigs. In this case, vertical closing loops are placed distal to the canines, Class II hooks are soldered, and the same anterior forces are used to retract the anterior teeth and correct the overjet as before.

The goals of denture completion are (1) to remove any first-order asymmetries, expansion, or lack of arch coordination, (2) to correct residual rotations, lack of root parallelism, torque deficiencies, and spacing, (3) to position the anterior incisors with artistic bends, and (4) to seat the canines and premolars with spurs and vertical elastics.

When the bands have been removed and retainers placed, the "recovery" period begins. In the context of this treatment perspective, a residual distal-axial inclination of the buccal segments along with an over-correction to disclusion of both the second molars and the distal cusps of the first molar will reduce the possibility of prematurities and occlusal trauma, permit the temporomandibular joint to function normally, and allow the teeth to settle into stable positions.

\section{Research Expectations}

The sample was divided into two groups based on the posttreatment retention protocol. One group received a maxillary Hawley retainer in combination with either a fixed mandibular canine-to-canine or first premolar to first premolar retainer. The mandibular fixed retainers were removed approximately 2 years posttreatment. The other group received maxillary and mandibular Hawley retainers. Our supposition is that there is less long-term postretention change in patients that were given temporary fixed retention than in those patients given only removable types of retention. 
The null hypothesis is: There was no statistical difference in malocclusion as measured at the pretreatment, posttreatment, and recall examinations in the fixed retention group compared to the removable retention group that received Hawley retainers only. 


\section{CHAPTER 4. RESULTS}

The sample used in the present study consisted of the diagnostic records from 166 orthodontically treated patients. All cases had recall records available at a minimum of 10 years posttreatment (Figure 4-1), and the average case was 16.0 years out of treatment ( $\mathrm{sd}=4.5$ years). Treatment records of these subjects had been collected from the offices of experienced orthodontic specialists from across North America who use standard Edgewise mechanics (Harris 1985). The mean age at the beginning of treatment was 13.9 years, with patients remaining in active treatment for an average of 2.9 years. Recall records were taken at an average 16.0 years posttreatment (Table 3-1).

The paramount question in this study was whether the type of retentioneither Hawley retainers or Hawleys in combination with a fixed lingual retainer-provided greater long-term stability. This raises the issue of whether the cases (those with a fixed retainer and those without) were comparable at the start of treatment. Table 4-1 lists the results of testing whether the chronological ages are comparable between the two groups. One-way factorial analyses of variance were used for each of the three examinations plus the two changes between examinations to test for comparability between the types of retention. Table 4-1 shows that none of the 15 F-ratios achieved statistical significance. This means that the chronological ages at the start and at the end of treatment were statistically equivalent between the two sorts of retention. The same holds for the ages at the long-term recall examination (about 16 years out of treatment). These tests show that, insofar as the age at treatment affects the amounts of growth during and after the active phase of treatment (e.g., McKinney and Harris 2001), the present samples are comparable. Any difference in outcomes would seem to be attributable to factors other than differences in the ages at treatment or at the follow-up examination.

\section{Cephalometric and Cast Analysis}

For the present study, we analyzed 32 variables (19 dental cast variables; 13 cephalometric variables). Descriptive statistics for the dental variables were generated for each of the five examinations (Appendix A), namely (1) status at the start of treatment, (2) status at the end of treatment, (3) status and the recall examination, (4) the in-treatment changes, and (5) the posttreatment changes. 


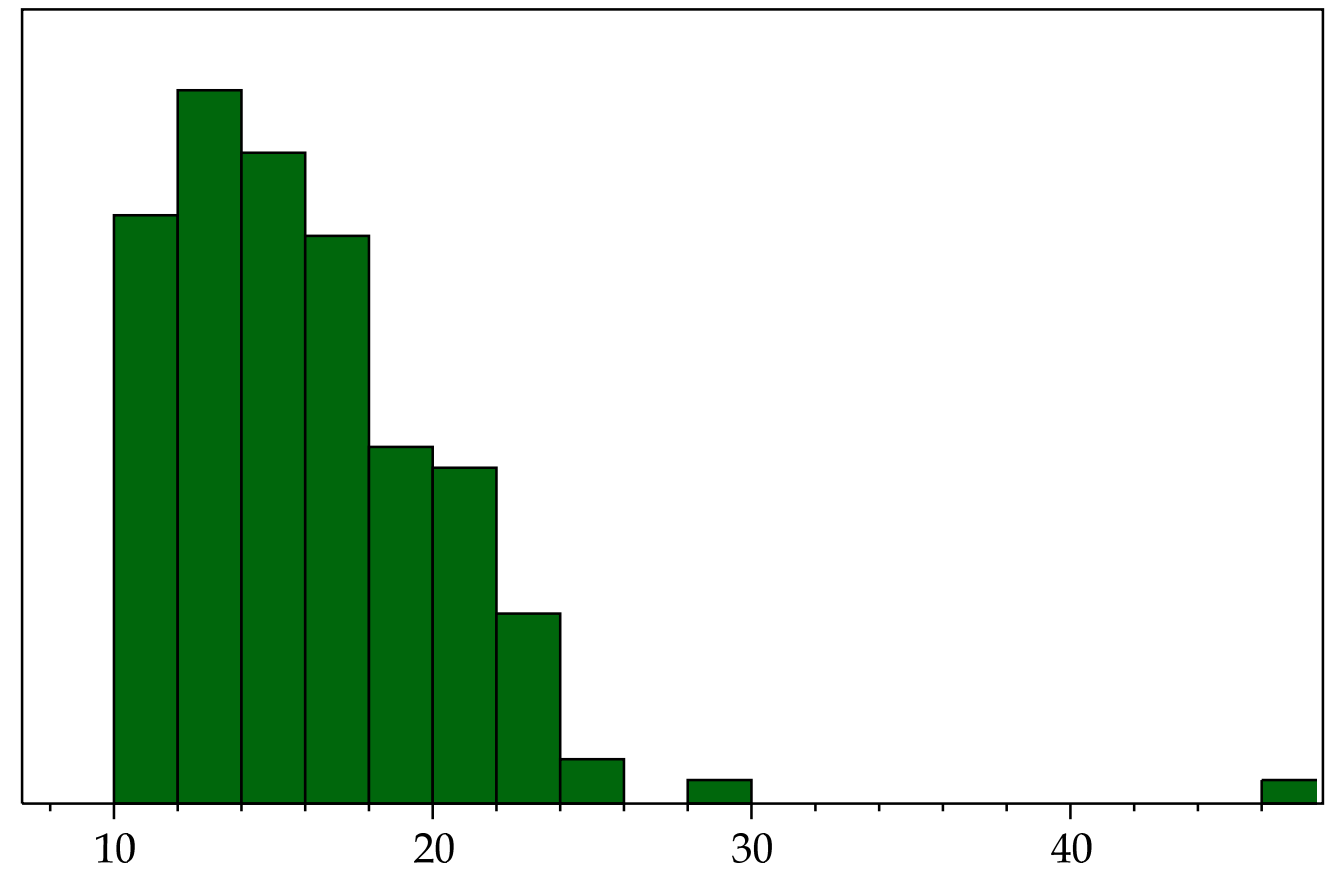

Figure 4-1. Distribution of the 166 subjects according to their years out of treatment.

We imposed a minimum of 10 years, which should allow most if not all of the relapse to occur (cf. Horowitz and Hixon 1969). The mean time out of treatment is 16 , but with the maximum being 46 years (i.e., the outlier on the far right).

Table 4-1. Results of one-way analyses of variance testing whether the chronologic ages are comparable between the types of retention (fixed or removable) at each of the three examinations and the intervals between them. ${ }^{1}$

\begin{tabular}{lccccccc}
\hline & \multicolumn{2}{c}{ Retention Type } & & \multicolumn{2}{c}{ Patient Sex } & & \multicolumn{2}{c}{ Interaction } \\
\cline { 2 - 6 } \multicolumn{1}{c}{ Variable } & F Ratio & P Value & & F Ratio & P Value & F Ratio & P Value \\
\hline Start Age & 0.57 & 0.4527 & 1.52 & 0.2189 & 1.00 & 0.3191 \\
End Tx Age & 0.12 & 0.7325 & 1.21 & 0.2730 & 1.35 & 0.2476 \\
Treatment Time & 3.36 & 0.0686 & 0.68 & 0.4100 & 0.18 & 0.6718 \\
Recall Age & 1.48 & 0.2260 & 0.70 & 0.4040 & 0.54 & 0.4651 \\
Time to Recall & 1.43 & 0.2332 & 0.00 & 0.9816 & 0.05 & 0.8306 \\
\hline
\end{tabular}

${ }^{1}$ There is $1 \mathrm{df}$ associated with each test. 


\section{Sexual Dimorphism}

The patient's sex is included in the statistical analyses because this aspect of the sample can have confounding effects on the interpretations if not controlled. Males are characteristically larger than females as a statistical average, males grow more during adolescence and for a longer time following adolescence than girls (e.g., Tanner et al. 1962; van der Linden 1986; Ursi et al. 1993), and so sexual dimorphism can influence the results if left uncontrolled. For several of the variables, such as dental arch dimensions, patient's sex is largely a nuisance variable, where its interpretation has no important clinical consequence. For other variables, such as whether one sex is more prone to relapse, knowledge of the patient's sex can provide clinical insights.

For each of the three examination periods, a two-way factorial analysis of variance was used as a screening device for each of the 32 variables to assess whether the type of retention (fixed versus removable) affects the results while controlling for patient's sex. The same was done for the in-treatment changes and the posttreatment changes. All of these results are listed in Appendix B. Again, "sex" was included to control for this known source of variation, and for completeness, we summarize the differences in sexual dimorphism in this section. Appendix $\mathrm{C}$ lists the results of testing whether the amount of change depends on the type of retention, with patient's sex and time-out-of-treatment as covariates. We list descriptive statistics for these variables in Appendix D.

Prior literature (e.g., Moorrees 1959; Knott 1961; 1972; De Koch 1972; Bishara et al. 1989) led us to expect that some dental arch dimensions would be statistically larger in boys at the start of treatment (Table D-1), but results show that most of the significant variables actually reflect the worse malocclusions in boys. Of the 17 significant variables, only maxillary intermolar width, mandibular intermolar width, and anterior segment perimeter are due to boys being larger than girls; the 14 other variables reflect access-to-treatment differences, where boys seeking orthodontic treatment have a more severe malocclusion than girls (Blair and Harris 2008; Glassell and Harris 2008). That is, epidemiologically, there are very few sex differences between boys and girls in the frequency or severity of malocclusion (e.g., Kelly and Harvey 1977). In contrast, most orthodontic practices are composed of a majority of girls (often with a sex ratio of 2-to-1) because girls (and their parents) are more aware of and more concerned with minor occlusal issues (Anderson 2010). The severity of the malocclusion has to be somewhat greater for boys to seek treatment.

This pattern is evident in these results (Table D-1), where, as examples, the mean status of overbite, overjet, ANB, AOBO, and the Curve of Spee are all larger (clinically more severe) in boys than girls. This suggests to us that minor 
occlusal issues are tolerated by boys (and their parents), whereas the "bar is lower" for girls, who are more apt to seek treatment for lesser malocclusions (e.g., Shaw 1981, 1991).

By the end of the active phase of treatment (Table D-2), most of the esthetic sex differences have been resolved. In concert, these adolescents now are older and have experienced the majority of their adolescent growth. At the end of treatment, the suite of significant male-female differences now is composed primarily of arch size variables, such as sex differences in arch lengths and widths. Because most of the posttreatment sex differences are due to sexual dimorphism (boys larger than girls), it appears that both sexes were treated orthodontically to similar posttreatment results. Also, it is not evident from these statistics that any of these cases was not treated to a clinically-acceptable outcome.

Table D-3 lists the 16 variables with significant sex differences at the longterm recall examination. Some of these (inter-canine widths, inter-molar widths) are due to the full-elaboration of sexual dimorphism towards the end of early adulthood $(\bar{x}=32.9$ years of age at T3), but others are due to sex differences in the nature of the posttreatment changes. These latter changes probably are some complex consequence of patients' failures to wear retainers in combination with sex differences in skeletal growth following treatment (Little et al. 1988).

While there was no significant difference in mean mandibular inter-canine widths or FMIA between the sexes at the end of treatment, significant differences do appear at the recall examination. Maxillary inter-canine widths, inter-molar widths, and anterior and posterior face heights remained significantly larger in males at the recall examination. Downs' occlusal plane remained steeper in females at the recall examination, but ANB and FMA became significantly larger in females as compared to the males. Two graphs are provided here to make this point. Figure 4-2 shows that FMIA is, on average, a significantly smaller angle in women than in men $(P=0.0011)$. Figure $4-3$ shows that the average FMA is significantly lower in men than in women $(\mathrm{P}=0.0200)$ at the recall examination. Ignoring these sex differences - even though they are no clinical consequencecan confound (distort) the statistical findings.

Review of Table D-3 shows that several of the occlusal measures (e.g., anterior and midarch discrepancies, and Incisor Irregularity) are not listed as being statistically significant. It is speculated that, by achieving similar posttreatment results, males and females can expect to see few significant occlusal differences between them, with the only differences being those attributed to size differences between the sexes. 


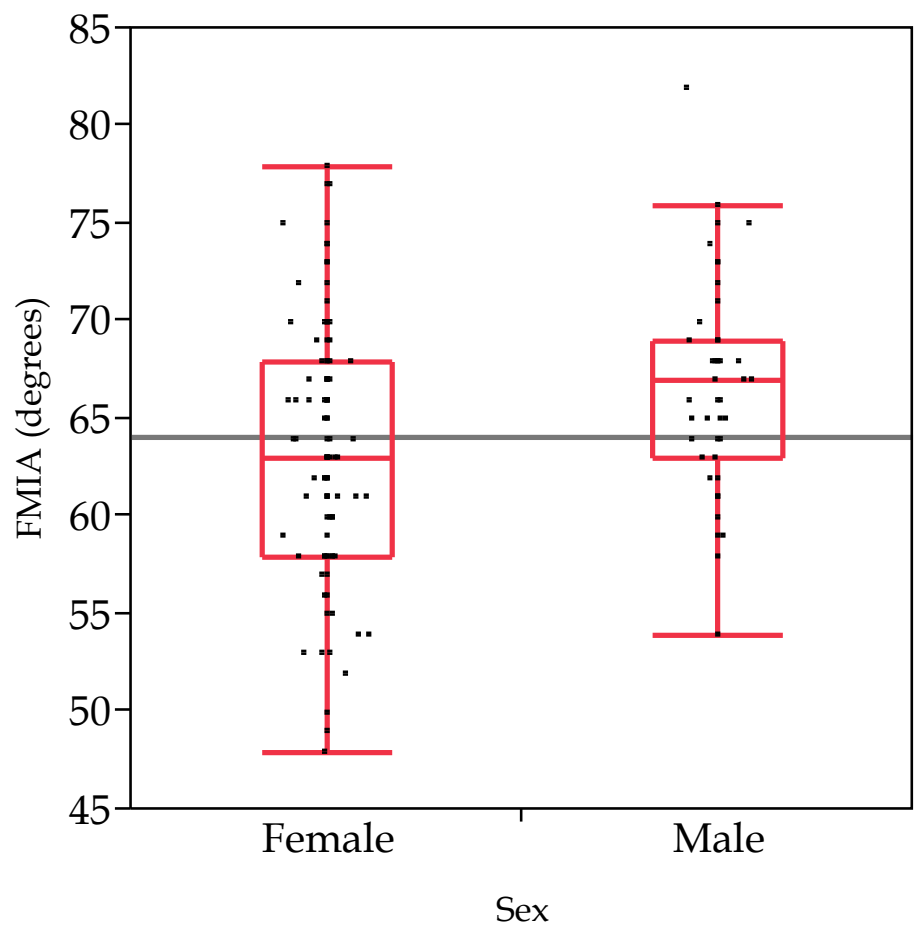

Figure 4-2. Box plot showing the sex difference in FMIA at the recall examination. 


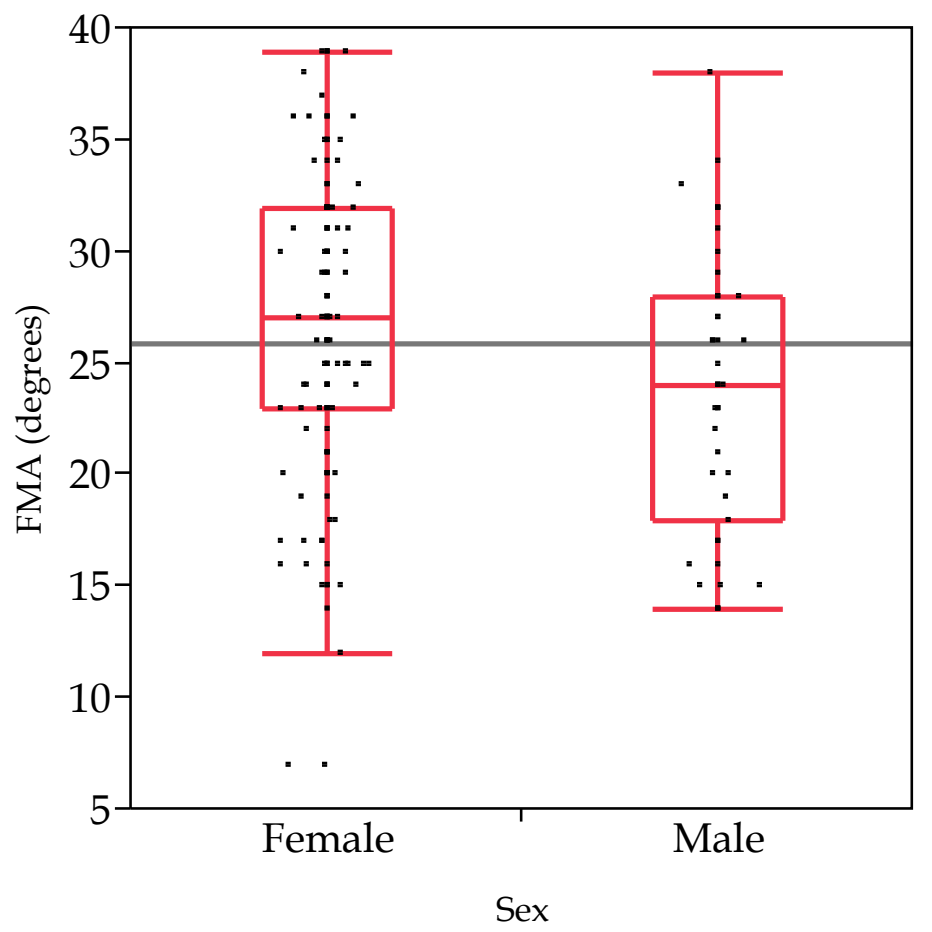

Figure 4-3. Box plot showing the sex difference in FMA at the recall examination. 
This inference raises an important question: There are roughly three times as many women as men in the sample (123 versus 43$)$, and it can only be hoped that those men that agreed to be recalled are representative of the sex at large. It is speculated that men are less concerned about their occlusions and, thus, relatively indifferent to undergoing a recall examination. There is, however, no practical way of testing this. This problem is common to all orthodontic-recall studies because there is no way to coerce former patients to participate.

Table D-4 lists the 17 variables that exhibit statistically significant sex differences in the amounts of in-treatment change. Most of these variables are dependent on tooth relationships. Except for incisor segment ratio and incisor segment depth, all of these variables (15 of 17) changed more in the boys than the girls. The theme seems to be that those variables that were more deviant in boys at the start of treatment (e.g., overjet, overbite, ANB, AOBO, Curve of Spee) warranted more correction during treatment. These changes are illustrated using the data for the AOBO discrepancy (Figure 4-4). The typical male orthodontic patient has a greater $\mathrm{AOBO}$ discrepancy at the start of treatment (perhaps because of differential selection of who actually receives treatment). Boys also experience a significantly greater in-treatment change, which leads to the sex differences noted here.

The 14 significant sex differences that occurred after treatment (Table D-5) are of particular interest since they constitute difference in the aging process (in combination with any relapse tendencies). Inter-canine width and inter-molar width, both in the maxilla and mandible, differed between men and women because women experienced greater relapse-where 'relapse' is constriction of these widths with age. Average changes were small-less than a millimeter. FMIA increased in both sexes, but significantly more in men.

FMA flattened about $2^{\circ}$ on the average in men, but the mean change was trivial in women (Figure 4-5). We speculate that this flattening is due to growth at the gonial process due to greater muscle force of the elevator muscles in males, and it may well reflect the continued decrease in FMA seen in childhood and adolescence (Ricketts 1972).

$\mathrm{SNB}$ - in combination with $\mathrm{ANB}$ and $\mathrm{AOBO}$ - changed a bit both in men and women, but in opposite directions. The change in SNB was positive in men $\left(\sim 0.6^{\circ}\right)$, but negative in women $\left(\sim 0.3^{\circ}\right)$. Since the change in SNA was statistically inconsequential in both sexes $\left(\sim 0.0^{\circ}\right)$, the increase in SNB (and in ANB) in men discloses that mandibular growth slightly exceeded that of the maxilla, making the skeletal profile a bit less retrognathic by the recall examination. In women, mandibular growth lagged slightly behind, creating a 


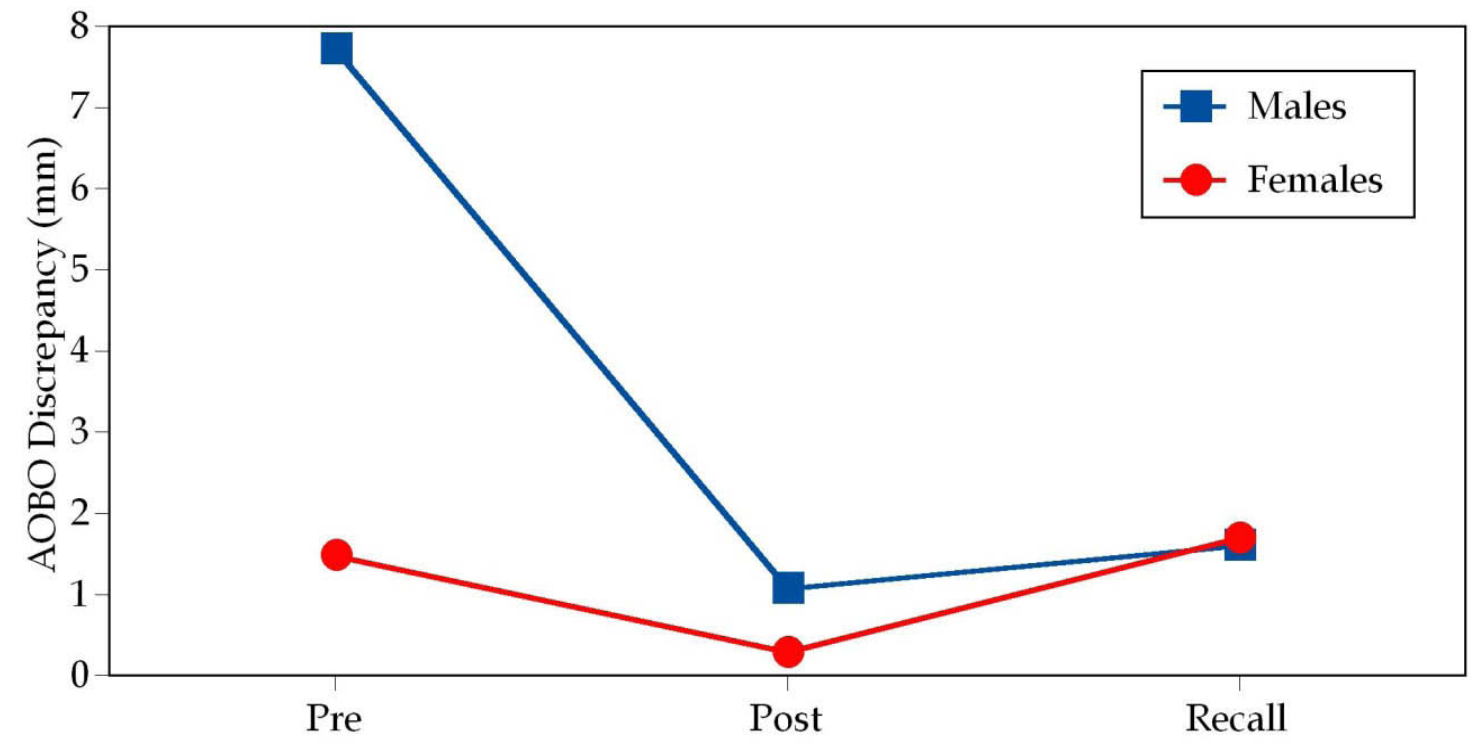

Figure 4-4. Plot of the average values of $\mathrm{AOBO}$ at the three examinations, by sex.

The point of interest here is that males who actually seek orthodontic treatment tend to have greater AOBO discrepancies than girls. Since the orthodontist is able to correct much of the skeletal discrepancy, the sex difference is considerably less at the posttreatment examination than at the start of treatment. This means that boys experience a significant greater in-treatment change for this variable than girls. 


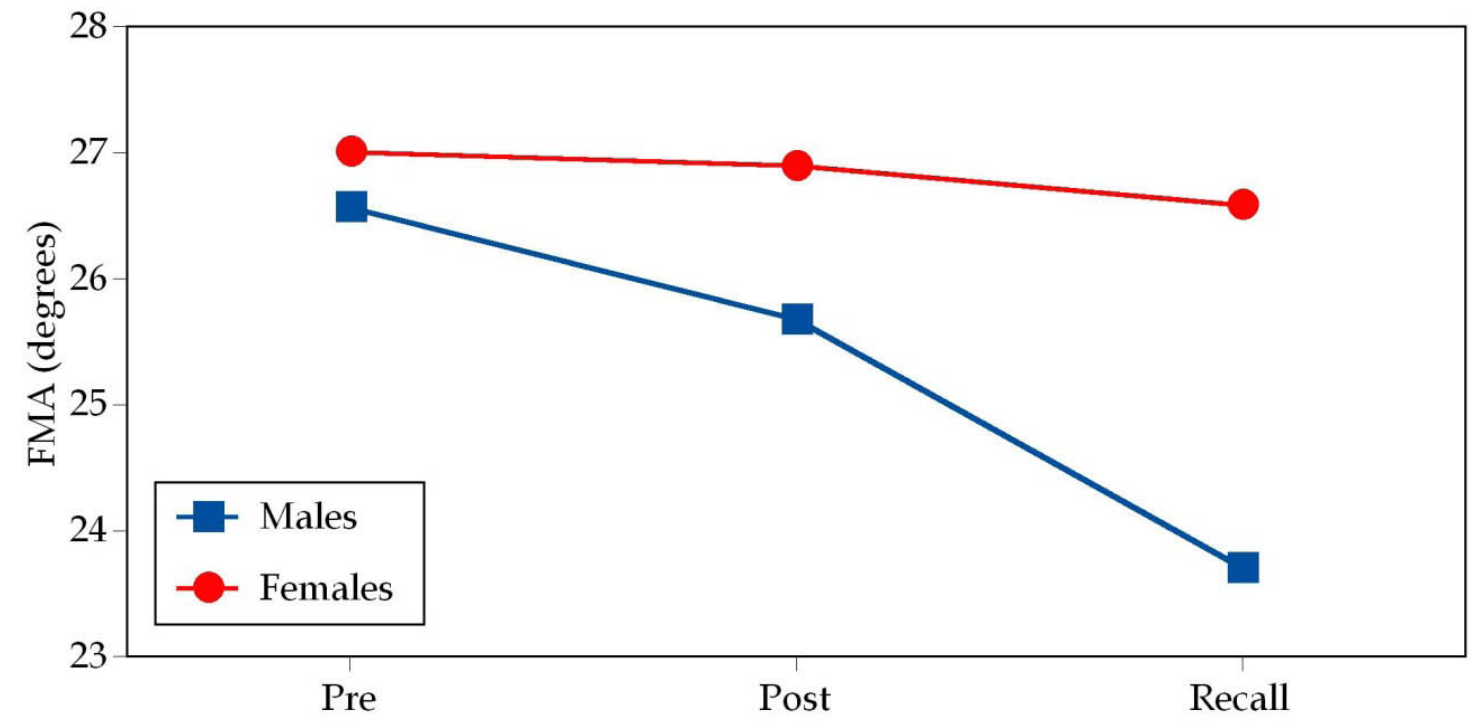

Figure 4-5. Plot of the average values of FMA at the three examinations, by sex. The mean changes are small across time in this cohort - and trivial in females. Males, however, experience a decrease over time of roughly 2 degrees, which, with the power of longitudinal analysis, is expressed as a statistically significant change. In other words, the male-female difference increases across the three examinations as these subjects grow and age. 
larger ANB at the recall examination. Clinically, it is unlikely that these subtle differences can be detected (e.g., Sloss et al. 2008).

\section{Retention Differences}

The central theme of this study, discussed in this section, is the effect of the kind of retention - either a removable Hawley or a fixed 3-3 or 4-4 retainerinfluenced the stability of the case when evaluated at long-term. As noted before, complete records are available on 97 and 69 cases, respectively. Appendix B lists the results of two-way analysis of variance that partitioned the sample by sex and type of retention. An ANOVA table was produced for each of the 13 cephalometric and 19 dental cast variables. These lengthy results are used to identify which variables exhibited statistical significance when considering the type of retention. Appendix A lists descriptive statistics for all variables separated according to type of retention. These statistics (sexes pooled) are supplied here so the nature of the significant differences can be interpreted. Variables exhibiting statistical significance are denoted with an asterisk.

We reviewed differences in the pretreatment variables with the intent of finding any variables that may have been thought informative by the orthodontist relative to the type of retention to be used when treatment was complete. That is, if differences exist at the pretreatment examination, this would suggest that the orthodontist had decided early-on, based on the nature of the malocclusion, to use a fixed appliance based on some distinguishing criteria.

Table A-1 lists the four variables (each denoted with an asterisk) with significant differences dependent on retention, and these are described as follows.

Overjet was appreciably greater in those subsequently treated with a fixed retainer. Average overjet at the start of treatment was more than $6 \mathrm{~mm}$ in the fixed group and slightly over $5 \mathrm{~mm}$ in the Hawley-only group.

Greater maxillary incisor procumbency is reflected too in incisor depth at the start of treatment, where cases with greater incisor depth $(\bar{x}=26 \mathrm{~mm})$ were more likely to be treated with fixed retention than those with shorter depths $(\bar{x}=$ $22 \mathrm{~mm})$.

The difference in SNA at the pretreatment examination was marginally significant as evaluated by the two-way analysis $(P=0.04)$, and it is likely that when the patient's sex is ignored, the retention difference would go away, even though the sex-by-retention interaction term was not significant. 
Total chin, which is mostly a soft tissue measurement but does include a portion of the bony chin, also was significant $(P=0.01)$. Total chin was greater at pretreatment (Figure 4-6) in the fixed group, although there was barely a millimeter difference between the two groups (14.74 and $13.67 \mathrm{~mm}$ ).

It is not likely that these differences at the pretreatment examination governed the orthodontist's decision concerning whether to use fixed retention at the end of treatment, but they do suggest that some occlusal conditions may influence the decision. It also is likely (Table A-1) that these differences evaluated at the start of treatment carried through to affect the posttreatment status - and that this latter condition controlled the decision about kind of retention.

More of the variables ( 7 denoted with an asterisk) were significantly different at the end of treatment (Table A-2). This in and of itself might indicate that orthodontists were attuned to which cases warranted fixed retention. Of note, incisor depth and overjet, which were significant at the pretreatment examination, persisted as significant predictor variables at the end of treatment. Incisor depth and overjet had been reduced in both groups during treatment, yet remained significantly greater in the fixed group at the end of treatment.

Total chin also continued to be statistically significant between groups at the end of treatment $(\mathrm{P}=0.02)$. The fixed retention group maintained a greater average value of $16.4 \mathrm{~mm}$ versus $15.4 \mathrm{~mm}$ in the removable group.

Incisor irregularity, which intuitively can be regarded as a proximate reason for using a fixed appliance, was greater in those receiving a fixed appliance (Figure 4-7), though the difference between the means seems small (0.5 $\mathrm{mm}$ and $0.2 \mathrm{~mm}$ ). Even though the mean difference was small, this difference is statistically significant. Those receiving a fixed retainer were more likely to exhibit greater Incisor Irregularity at the end of treatment. Overbite also was significantly larger in the fixed group at the posttreatment examination.

Table A-2 lists the Curve of Spee and reveals that the average curve is small in both groups (Figure 4-8), but the value is actually almost twice as great in the fixed group $(0.8 \mathrm{~mm})$ as in the removable group $(0.4 \mathrm{~mm})$. While the mean difference between the two groups is trivial $(\sim 0.31 \mathrm{~mm})$, the distributions show that cases with deeper curves are more likely to be fitted with a fixed retainer.

Molar correction also occurs on this list (Figure 4-9). The key point is that the median for the removable group is appreciably closer to zero (closer to a Class I relationship) than the median of the fixed group, meaning that cases in 


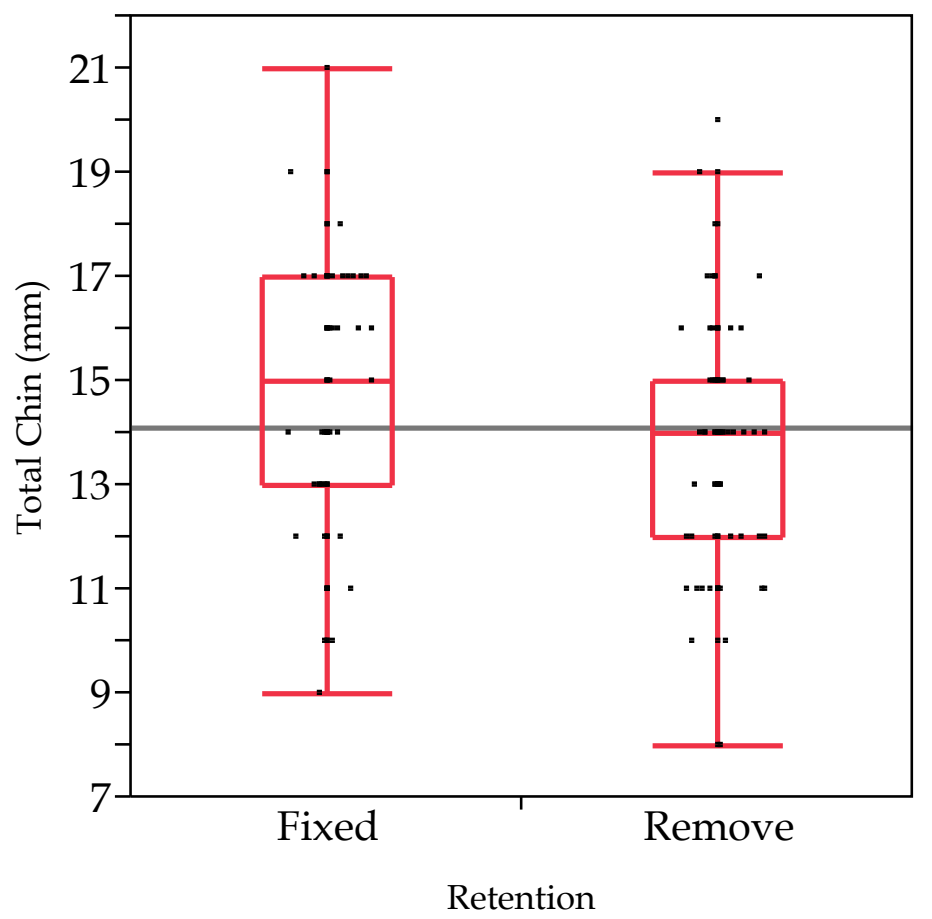

Figure 4-6. Box plot of "total chin" at the pretreatment examination based on type of retention.

This dimension is significantly greater in the sample that would receive a fixed retainer at the end of treatment. 


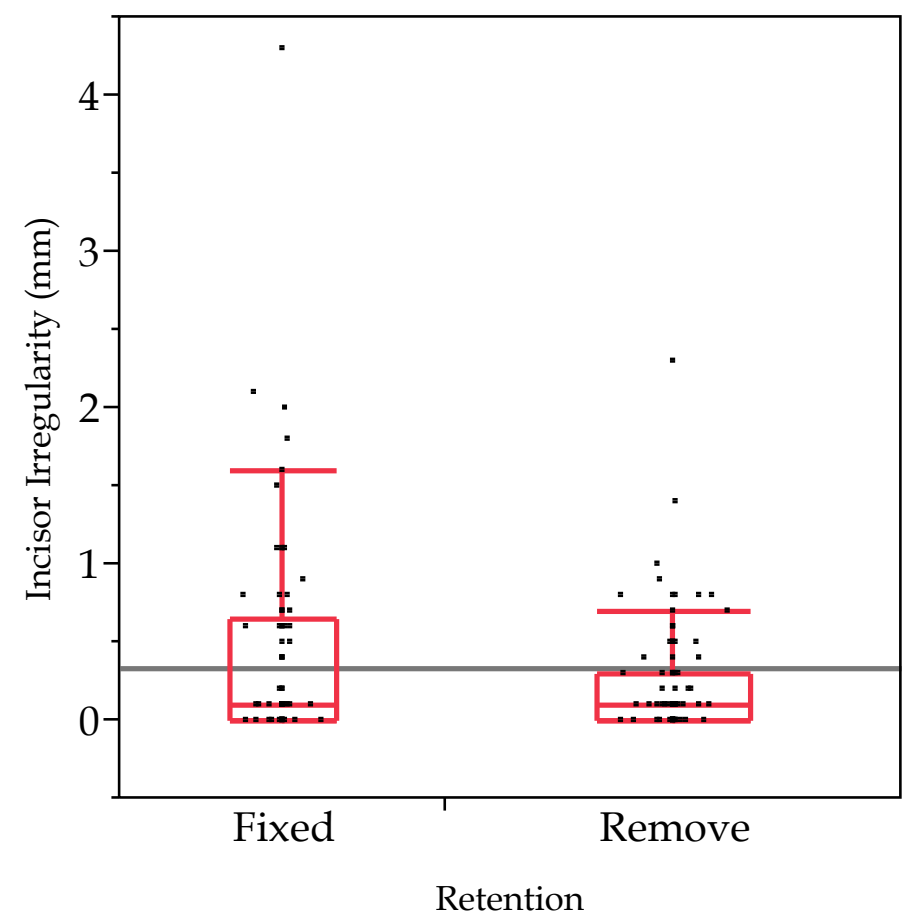

Figure 4-7. Box plot of the distribution in mandibular Incisor Irregularity at the end of treatment according to the type of retention.

Both samples are positively skewed, since "negative" Incisor Irregularity has no definition. Note that cases with greater irregularity $($ e.g., $>1 \mathrm{~mm})$ are more common in the fixed sample. 


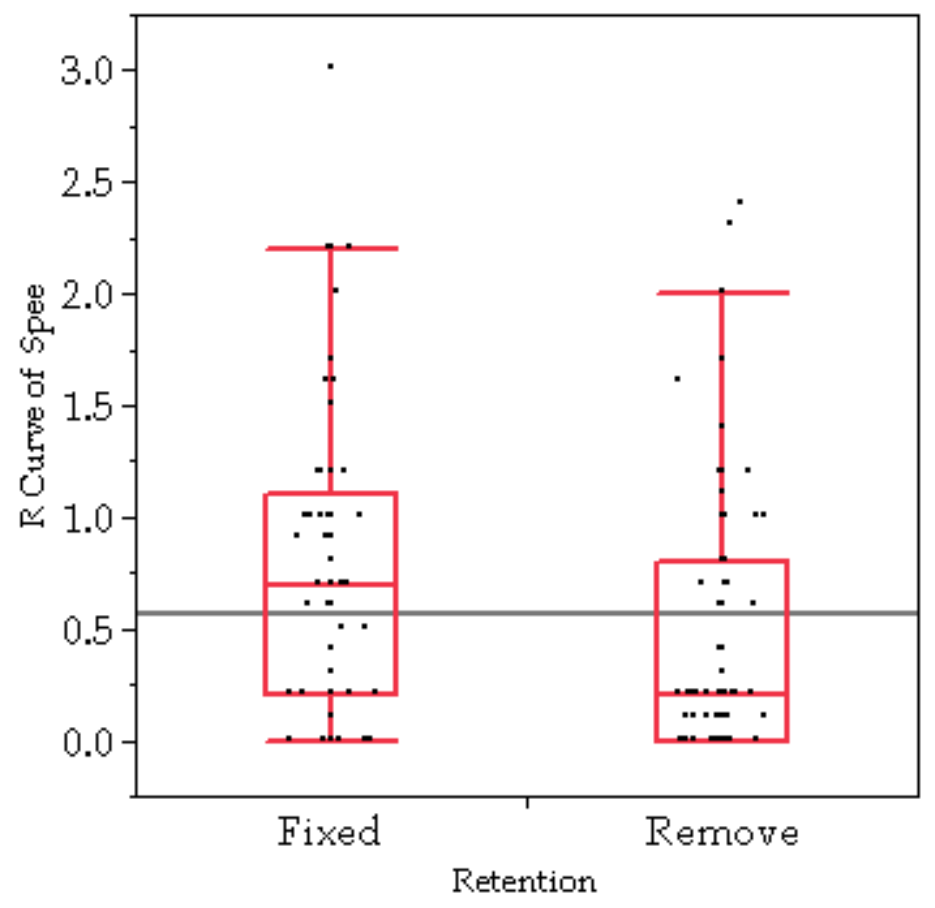

Figure 4-8. Box plot of the distribution of the Curve of Spee at the end of treatment in the two retention groups. 


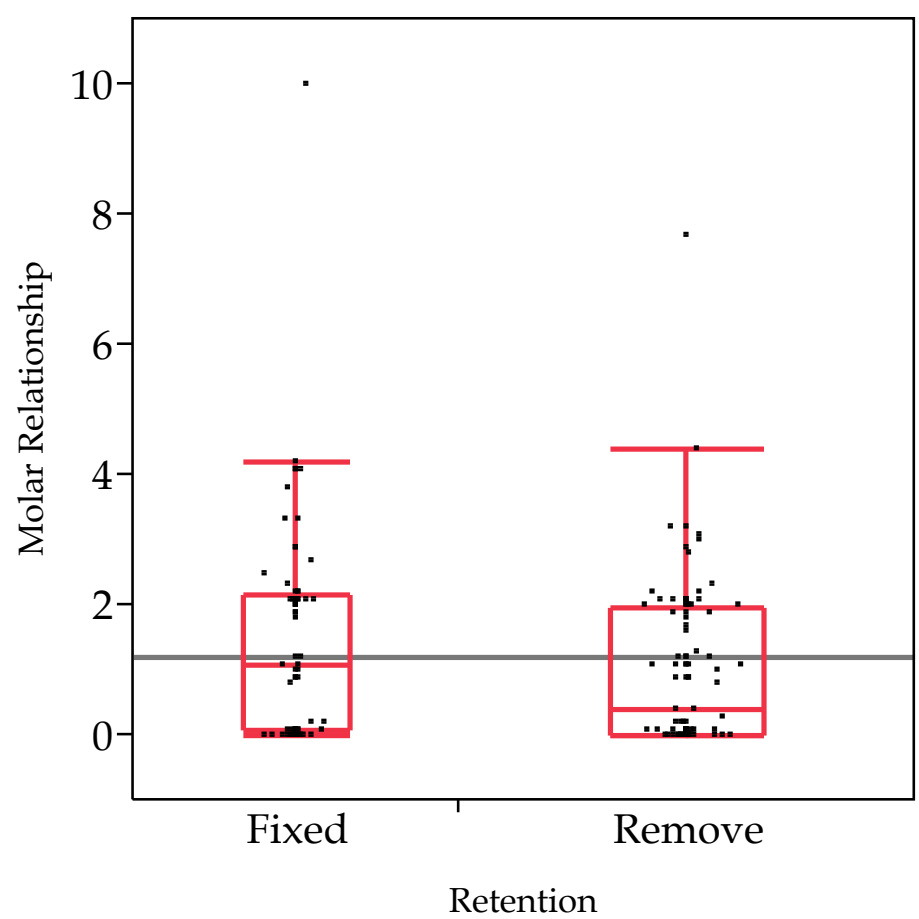

Figure 4-9. Box plot of molar relationship at the end of treatment in the two retention groups.

Notice that 2 cases, one in each group, were treated to a Class II molar relationship, which accounts for the outliers at the top of the graph. 
which a Class I relationship was not fully achieved during treatment were more likely to be put into a fixed retainer.

Analysis of the long-term recall records (Table A-3) reveals four variables that are predictive of the kind of retention used. The Curve of Spee continues to be an important predictor throughout the recall period. There was a moderate increase in the Curve of Spee in the fixed group (Figures 4-10 and 4-11), whereas it nearly doubled in the removable group following treatment.

According to the probability values, Incisor Depth exhibited the largest statistical difference between the two groups (Figure 4-12), with a greater depth $(24 \mathrm{~mm})$ in the fixed group than the removable group $(20 \mathrm{~mm})$. This finding was unanticipated and difficult to explain.

It is interesting that total chin no longer exhibits significance at the recall examination. The difference between these two groups was relatively the same at the start of treatment, persisted throughout treatment, but then disappeared years following orthodontic treatment. This suggests that orthodontics had little effect on this variable, and that with the normal growth and development that typically follows treatment, the previous statistical differences seen in this measurement were resolved in time.

Mandibular Incisor Irregularity exhibits a statistically significant difference between groups at the recall examination $(\mathrm{P}=0.0104$ by two-way ANOVA; Figure 4-13). This is a key finding since Incisor Irregularity often is used as an important measure of stability because it is so obvious to the patient. The mean of the fixed group was $1.92 \mathrm{~mm}$ and the mean of the removable group was $2.53 \mathrm{~mm}$. However, clinically this difference is pretty small and amounts to only about $0.6 \mathrm{~mm}$. One additional potential benefit of fixed retention, as evident from Figure 4-13, is that visually, the fixed retainers kept outliers to more of a minimum, as you can see that the points in the fixed group are more closely related to the median than those of the removable group. This proposed benefit is purely conjecture and might be difficult to support statistically.

The fourth variable that differed significantly at the recall examination (Table A-3) is IMPA, and this is graphed in Figure 4-14. By two-way ANOVA, the $\mathrm{P}$ value is 0.0302 . However, the difference is not visually apparent in the graph, where the mean of the group with fixed retention $(\bar{x}=91.42)$ is significantly higher than in the Hawley-only group $(\bar{x}=89.45)$. In other words, the lower incisors are a bit more flared in the group with fixed retention. It was difficult to determine why this difference occurs at the recall examination, as this difference was not present at the end of treatment. 


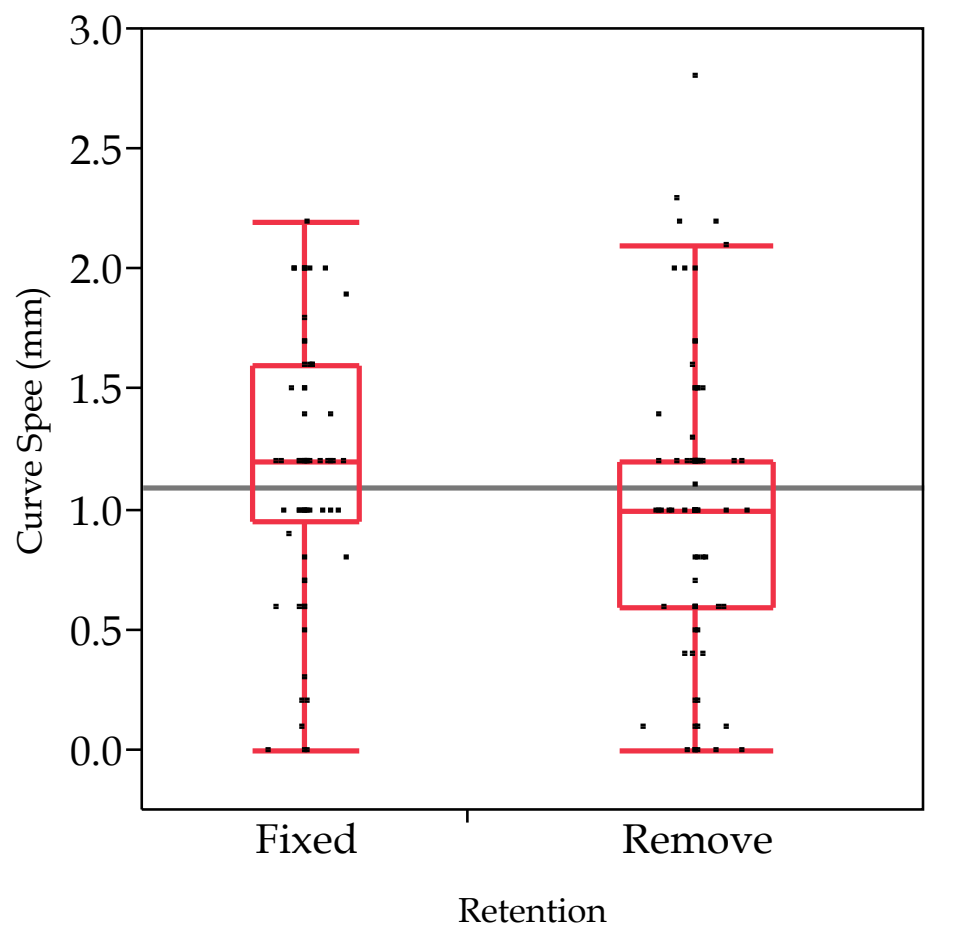

Figure 4-10. Box plot of the distribution of Curve of Spee at the recall examination by type of retention.

Recall that the Curve was deeper at the end of treatment, and these data show that the difference between groups persists for at least a decade thereafter. 


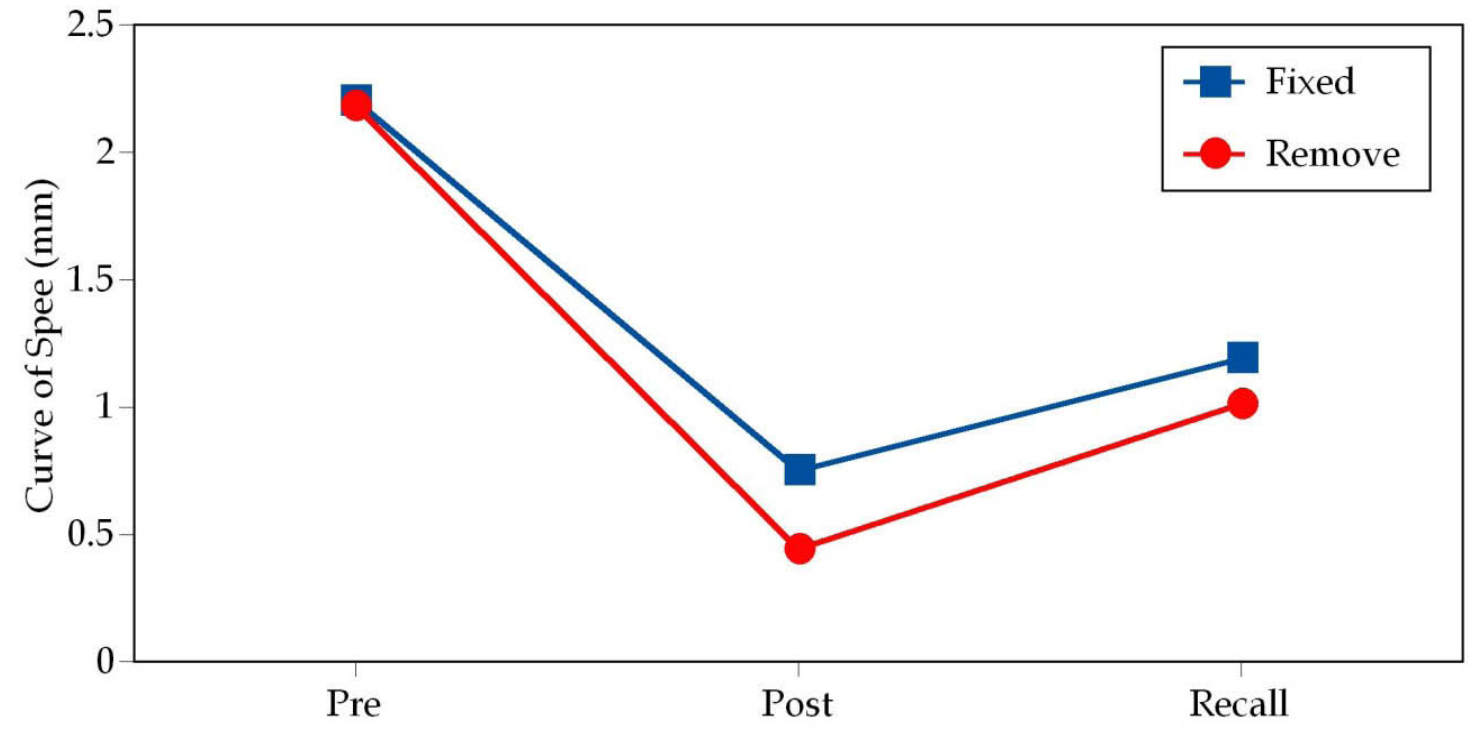

Figure 4-11. Plot of mean Curve of Spee by examination.

Those with greater depths at the end of treatment tended to be the cases in whom fixed retention was used. Note that difference between groups persists into the recall examination, so there was no posttreatment self-correction of the condition. 


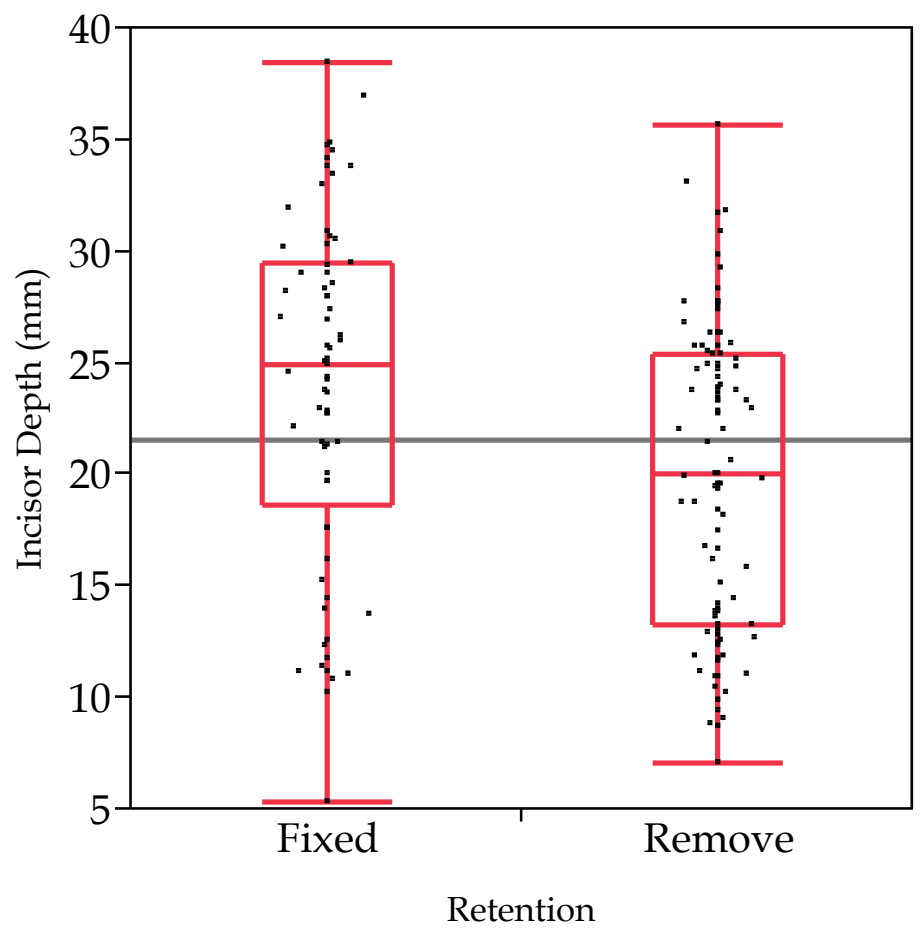

Figure 4-12. Box plot of Incisor Depth at the recall examination by retention group.

This depth is significantly greater in the sample where fixed retention was used. 


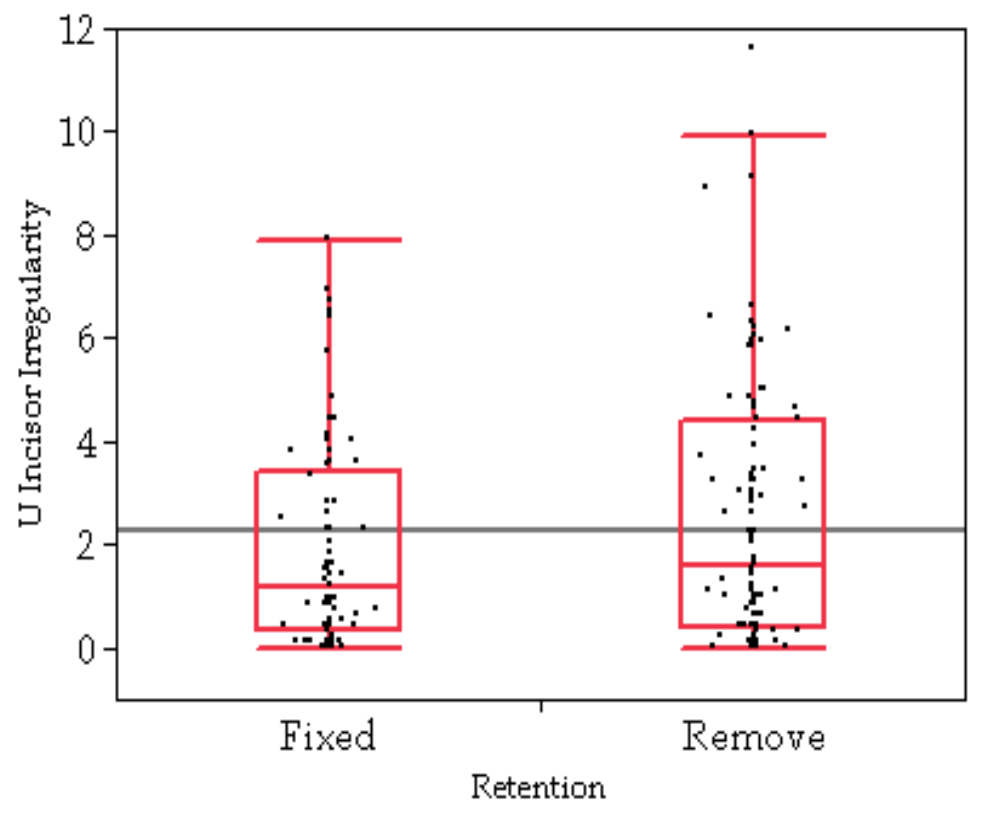

Figure 4-13. Box plot of the distributions of mandibular Incisor Irregularity at the recall examination according to type of retention.

The difference between groups is not obvious in this plot, but average irregularity is $\sim 0.6 \mathrm{~mm}$ greater in Hawley-only group. 


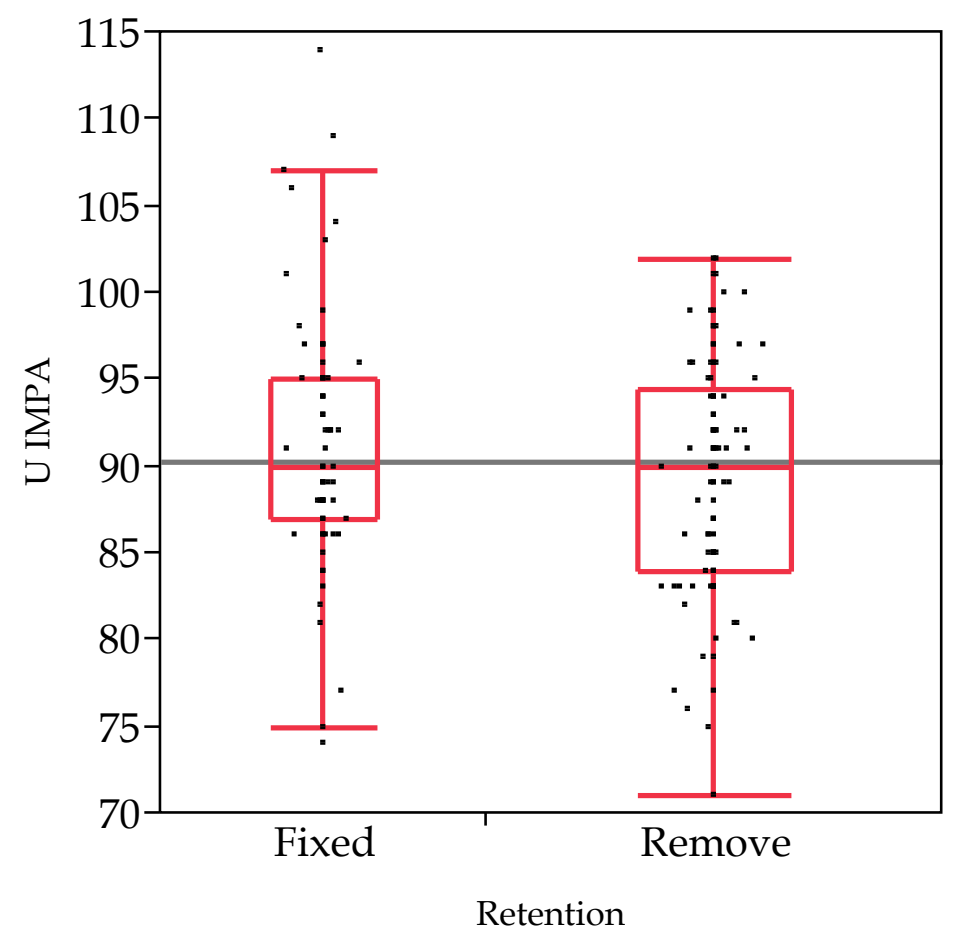

Figure 4-14. Box plot of the distributions of IMPA at the recall examination. By ANOVA, there is a significant difference between groups $(P=0.0302)$, with the fixed group having greater flaring of the lower incisors. 
Table A-4 addresses the question of which variables changed differently during the course of treatment depending on the type of retention used. The Curve of Spee changed more during treatment in the removable group. There was no difference at the start of treatment; both groups averaged $2.2 \mathrm{~mm}$ of curve, so the difference is due to the orthodontists' effectiveness in flattening the curve during treatment. In other words, less correction of the Curve of Spee enhanced the likelihood that the orthodontist would use fixed retention.

Incisor segment ratio appears as a significant predictor based on the intreatment changes. This variable (ISR) increased only slightly during treatment $(\sim 0.1)$ in the fixed group, but increased substantially $(\sim 2.3)$ in the removable group.

Six variables achieved statistical significance when the posttreatment changes are evaluated (Table A-5). The question addressed here is: Which variables changed ("relapsed") more in one group than the other following the active phase of treatment? Maxillary intermolar width is one such variable. Intermolar width decreased less than a millimeter on average in both groups, but significantly more in the removable group (ca. -0.4 versus $-0.8 \mathrm{~mm}$ ).

The anterior discrepancy increased slightly in both groups, but the increase was almost double in the removable group (ca. 0.5 versus $0.9 \mathrm{~mm}$ ). Interestingly, incisor segment ratio, which substantially increased in the removable group during treatment, decreased $(-0.8)$ during the posttreatment examination period versus a continued increase $(\sim 0.7)$ in the fixed group.

Perhaps the most notable changes observed during the posttreatment period were seen in Incisor Irregularity (Figures 4-15, 4-16 and 4-17). During the average of $\sim 16$ years between the posttreatment and recall examinations, mandibular Incisor Irregularity increased an average of $\sim 1.5 \mathrm{~mm}$ in the fixed group and $2.3 \mathrm{~mm}$ in the removable group. This is suggestive evidence that fixed retention does inhibit long-term relapse even though it is only in place for the first two years following treatment. That is, one could argue that the lessened amount of relapse following treatment is due to the interval of fixed retention. While marginally significant statistically $(P=0.0247)$, though, the difference between the groups averages only $0.8 \mathrm{~mm}$, so even if true, the effect of fixed retention is very small and certainly not of any real clinical consequence.

Incisor segment depth (ISD) is the one other variable that achieved statistical significance (Table A-5). This marginally significant difference between groups $(P=0.0315)$ occurred because there was virtually no change after treatment in the group with fixed retention $(\bar{x}=-0.1 \mathrm{~mm})$ but the Hawley- 


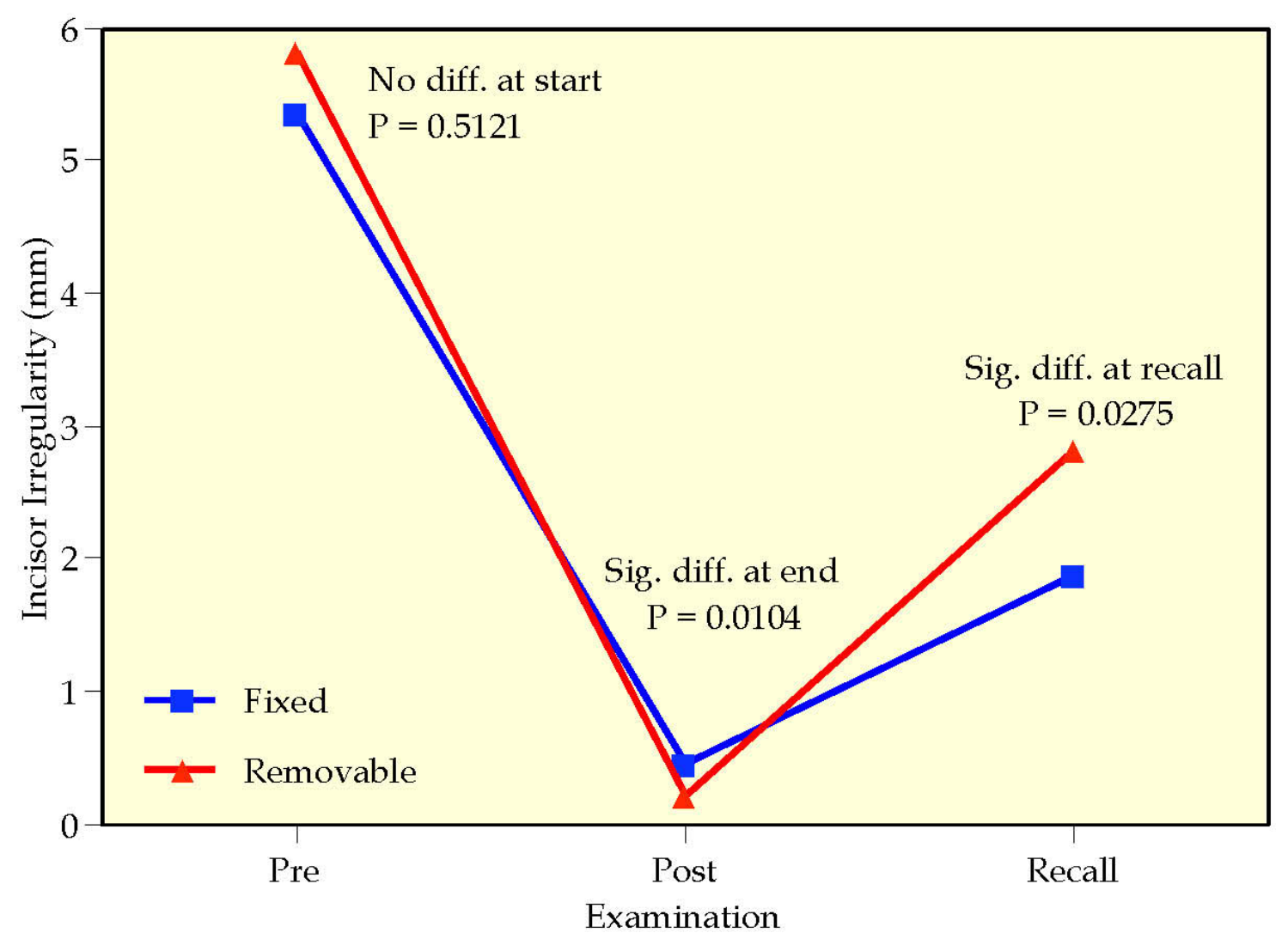

Figure 4-15. Plot of the mean values for Incisor Irregularity by examination and kind of retention.

A key issue is that irregularity is statistically smaller at the recall examination in the fixed group, the average difference is only about $1 \mathrm{~mm}$. 


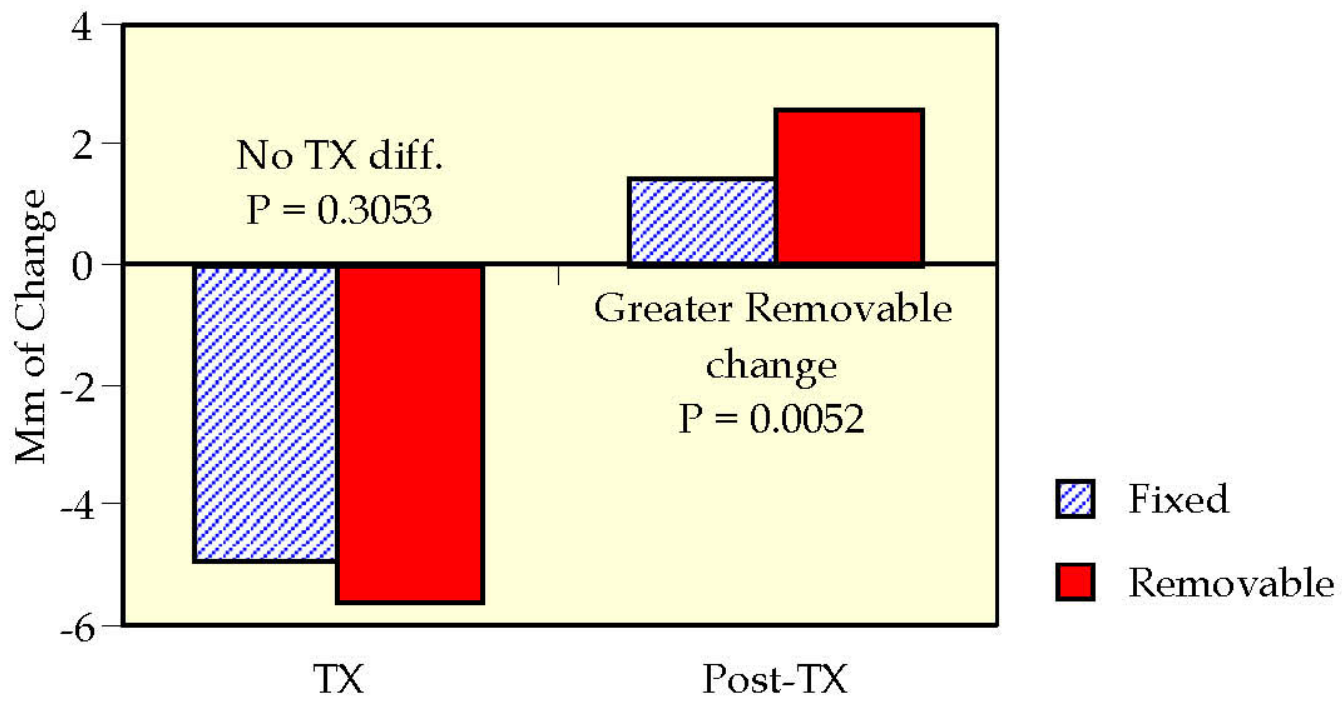

Figure 4-16. Bar chart of the average changes in Incisor Irregularity by type of retention.

The Hawley-only group relapsed significantly more following treatment, though, clinically, the mean difference between groups is only $1.2 \mathrm{~mm}$. 


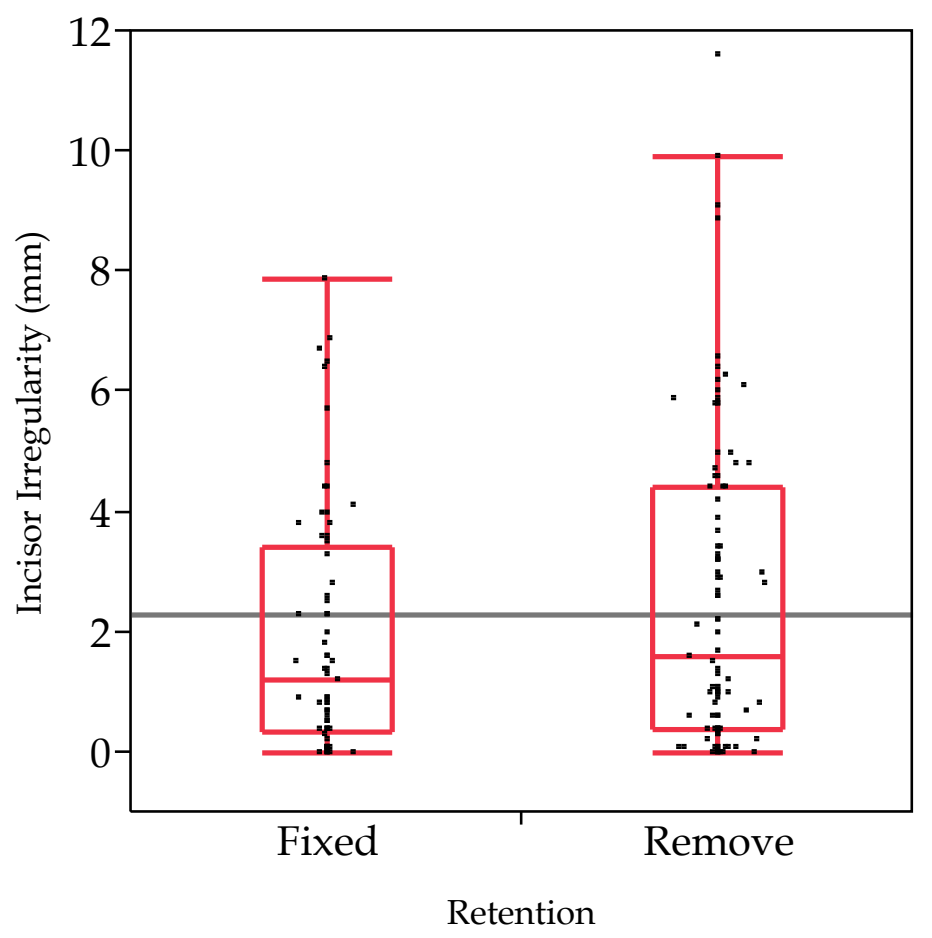

Figure 4-17. Box plot of Incisor Irregularity at the recall examination, by kind of retention.

In the fixed group, $25 \%$ of the cases (17/69) exceed the clinically-acceptable limit of $3.5 \mathrm{~mm}$ suggested by Little. This contrasts with $29 \%$ in the removable group (28/97). This difference is not large, but it is suggestive that the interval of fixed retention has a long-term benefit in at least some patients. 
only sample changed significantly more $(\bar{x}=0.3 \mathrm{~mm})$ - though the change probably is of no clinical consequence.

\section{Multivariate Analysis}

The prior results, looking at the variables one by one, do not suggest which variables are the most influential. In this section, stepwise discriminant functions analysis (e.g., Cooley and Lohnes 1971) was used to identify which of our set of cast and cephalometric variables evaluated at the end of treatment were most influential in distinguishing between those cases who received a fixed retainer compared to those who were given Hawley retainers alone. In other words, which variables observed by the orthodontist at the end of the active phase of treatment might have influenced the choice of whether to place a fixed retainer? Discrimination functions analysis was used to select just the subset of variables that maximally discriminate between the two kinds of retention.

As an initial screening, all posttreatment variables $(\mathrm{k}=32)$ were input and evaluated in a stepwise fashion, and four of these were entered with $P$ values less than the conventional alpha level of 0.05 . These are, in order of entry, (1) Incisor Depth, (2) Overbite, (3) Total Chin, and (4) Incisor Irregularity. (As an aside, prior probabilities were assumed to be equal, the variables were treated as linear rather than quadratic, and a common covariance matrix was assumed.)

Figure 4-18 is a plot of the means of the four variables that maximally distinguish between the two sorts of retention. It happens that each of these variables has a larger average value in the sample treated with a fixed retainer. This is not anticipated, but it does make interpretation simple. Evaluated at the end of treatment, the most-distinguishing variables are: (1) greater incisor depth, (2) greater overbite, (3) greater total chin dimension, and (4) greater Incisor Irregularity. This is the statistical result, but it is suggestive that the orthodontist was attuned to at least most of these differences.

Just these four variables then were input into a second analysis. Using all four variables, the discriminant functions correctly classified $72 \%$ of the cases as to the type of retention (105/166). The canonical plot (Figure 4-19) shows that there is considerable scatter among cases, but that Incisor Depth and Overbite are the important discriminators along the first canonical axis. Cases with greater Incisor Depths and deeper Overbites were much more likely to receive a fixed retainer. The other two variables, Total Chin and Incisor Irregularity, contribute statistically (but less) to the discrimination between groups. It is of note that Incisor Depth - a variable undocumented in most studies - is shown to be the single most discriminatory variable. Clinically, Incisor Depth (Figure 3-5) is 


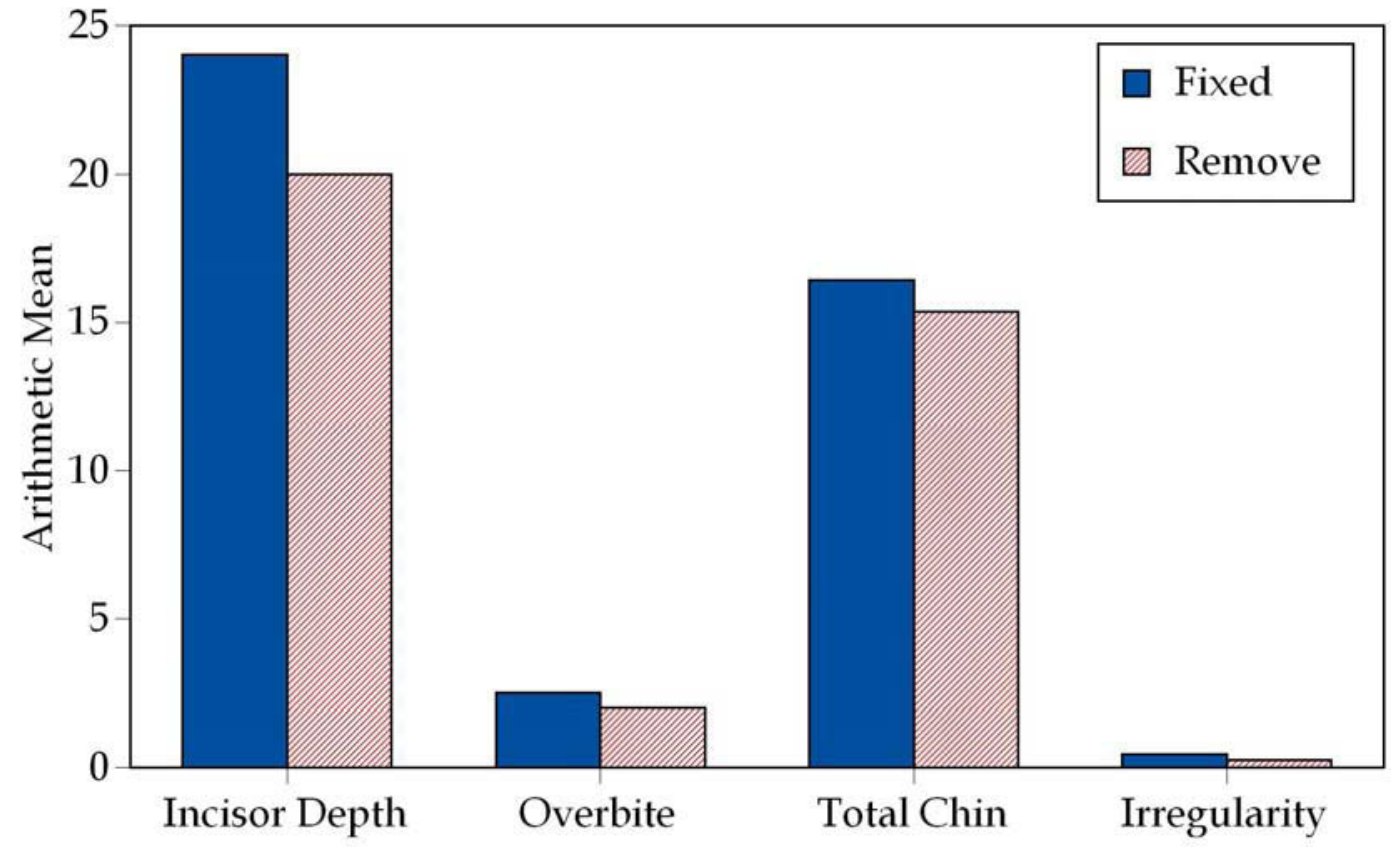

Figure 4-18. Bar graph of the means of the four discriminating variables, by group.

Cases receiving a fixed retainer at the end of treatment tended to have higher values for each of these four dimensions, so it is suggestive that the orthodontist was sensitive to these conditions when deciding how a case ought to be retained. 


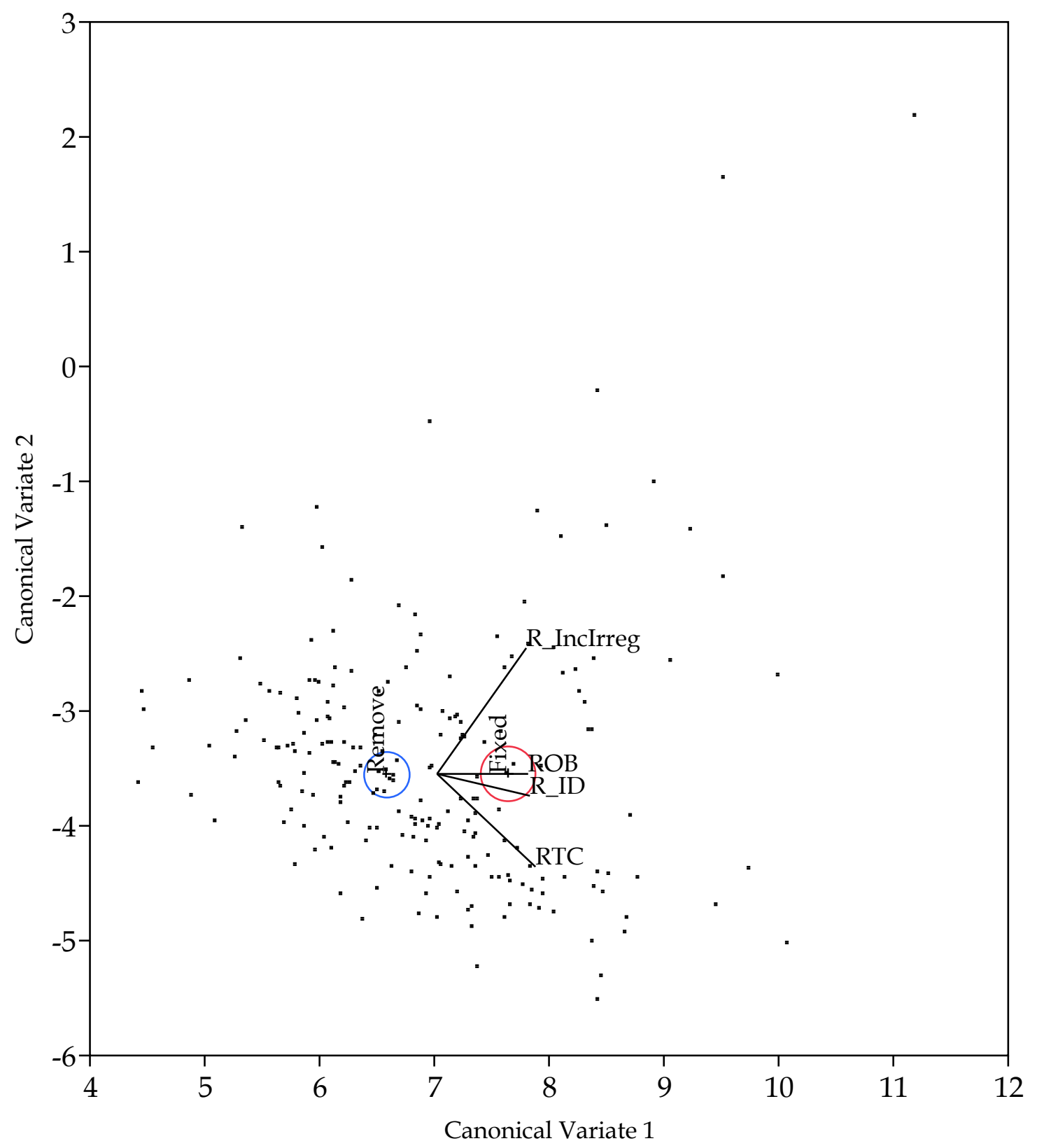

Figure 4-19. Canonical plot of the first two variates that discriminate between the groups treated with a fixed versus just-Hawley retainers.

These canonical variates are weighted combinations of the four discriminatory variables that maximally distinguish between the two groups. Based on all cast and cephalometric variables at the end of treatment, four dimensions correctly predict kind of retention they received in $72 \%$ of the cases. 
primarily a measure of how flared (proclined) the maxillary incisors are when viewed occlusally. When the incisors are upright and essentially perpendicular to the occlusal plane, Incisor Depth will be minimized, so greater Incisor Depth in the fixed-retention sample suggests that orthodontists were somehow attuned kind of retention they received.

\section{Amount of Relapse}

The prior sections identified several variables that exhibit statistically significant differences between the types of retention. In this section, we turn attention to assessing the effect that variables had on the extent of relapse. Appendix $\mathrm{C}$ lists the results of testing each of the 32 variables to evaluate whether the amount of change depends on the type of retention, with the patient's sex and time out of treatment as covariates.

A word of explanation will help clarify the thought behind these statistical tables. Retention (Hawley versus fixed) is the key variable, and the other variables in the model are used simply to control for known sources of confounding variation. "Sex" was included merely to absorb the variance due to men being larger than women. Time (i.e., years out of treatment) was included to control for the variability in the duration of time available for relapse. In fact, "time" is rarely significant because most relapse seems to occur soon after treatment (Horowitz and Hixon 1969), so, at a minimum of one decade out of treatment the full rebound due to treatment probably has occurred. The other four terms in the model are the standard first- and second-order interaction terms (e.g., Winer et al. 1991) that are included to check for additivity of the main effects. Put simply, additivity - that the main effects respond in proportional fashions - is a necessary assumption; if additivity does not occur (i.e., a significant interaction effect) then the model is biased and yields unreliable results.

Of the 32 variables, six showed a significant relationship between the amount of relapse and the kind of retention. Figures 4-20 through 4-25 provide graphic representations of the group differences for these six variables.

Anterior discrepancy (Table C-1) exhibits a statistically significant difference depending on type of retention $(\mathrm{P}=\sim 0.02)$. Figure 4-20 discloses that using fixed retention provides greater stability, although clinically small, following treatment.

Mandibular Incisor Irregularity (Table C-17) shows up as a significant variable $(\mathrm{P}=\sim 0.01)$. Figure $4-25$ shows that the sample with fixed retention was 


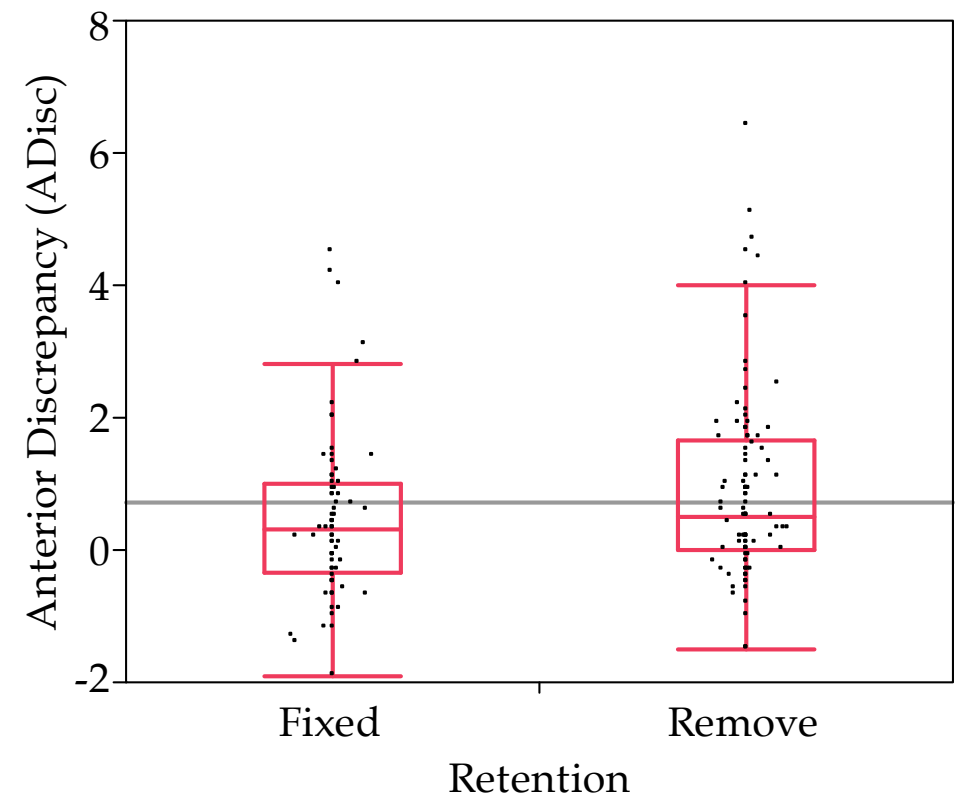

Figure 4-20. Box plot of the amount of relapse in Anterior Discrepancy (ADisc) dependent on type of retention.

On average, treatment change in anterior discrepancy was significantly greater in the removable sample, though clinically less than $1.0 \mathrm{~mm}$. 


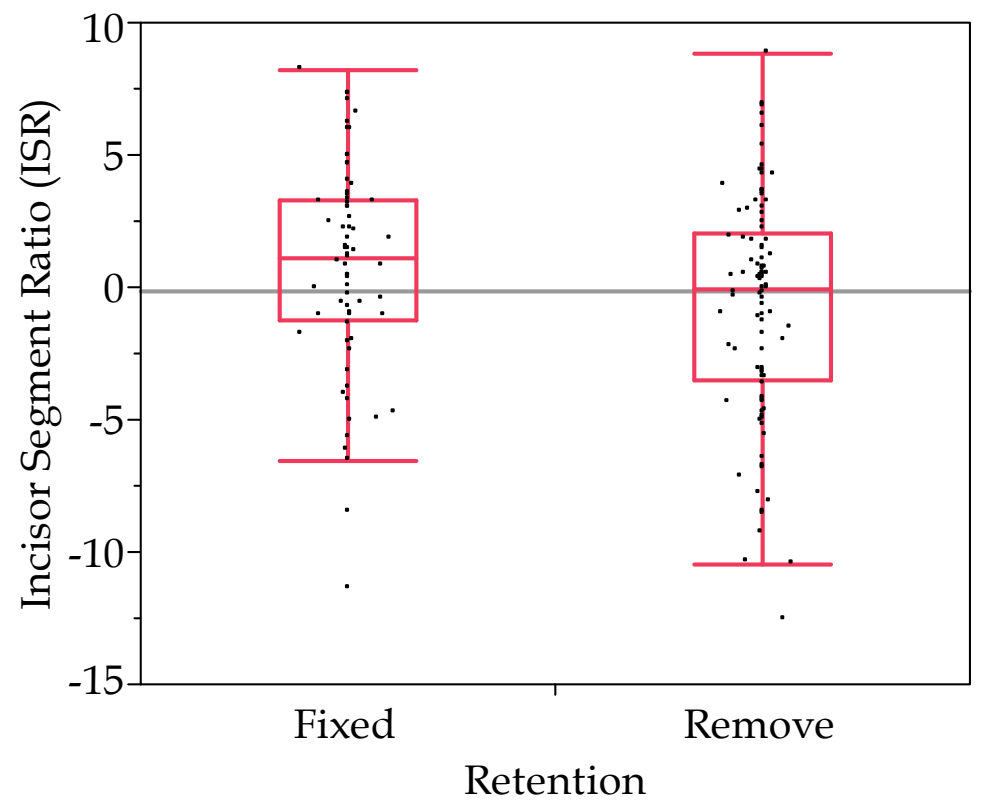

Figure 4-21. Box plot of the amount of relapse in Incisor Segment Ratio (ISR) dependent on type of retention. 


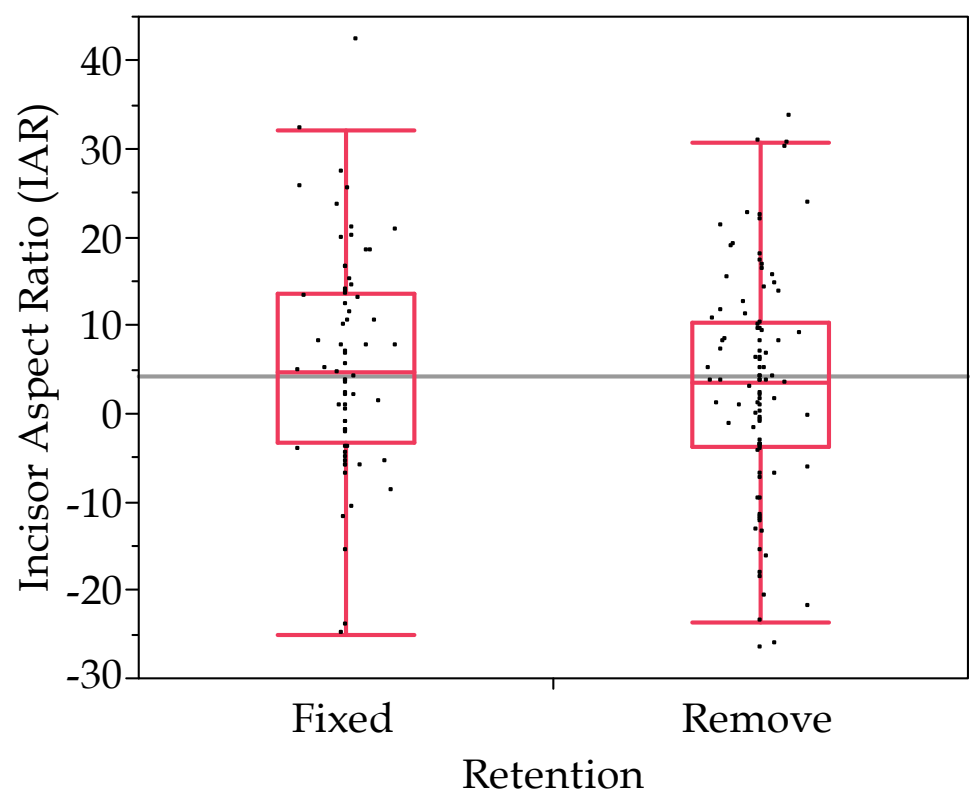

Figure 4-22. Box plot of the amount of relapse in Incisor Aspect Ratio (IAR) dependent on type of retention. 


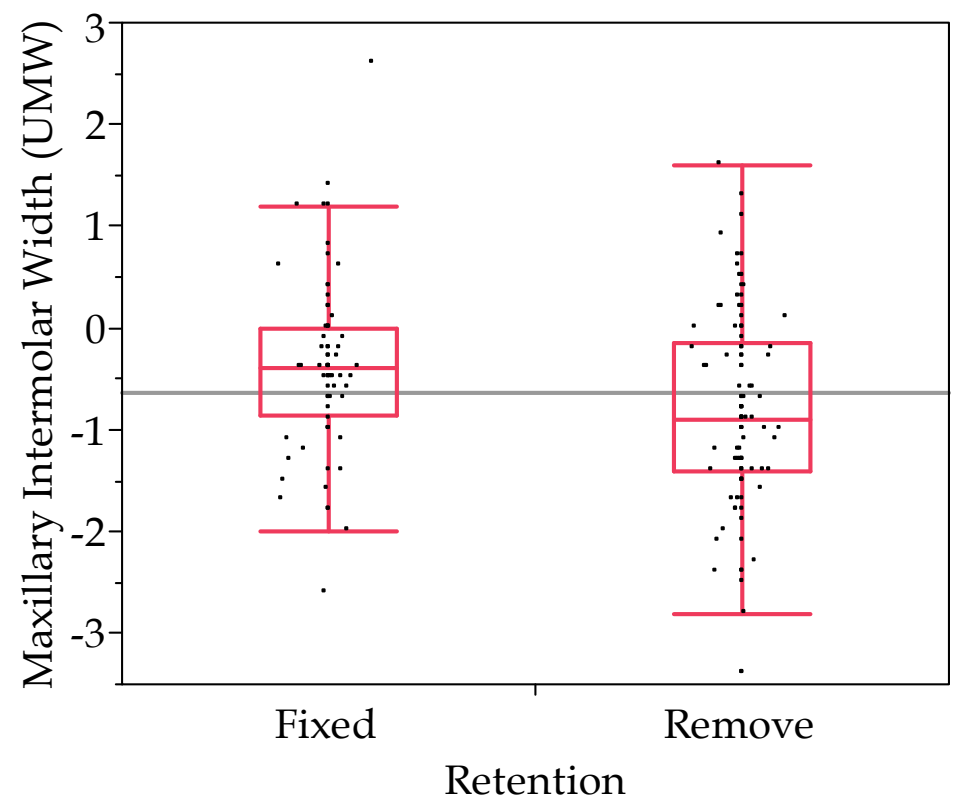

Figure 4-23. Box plot of the amount of relapse in Maxillary Intermolar Width (UMW) dependent on type of retention. 


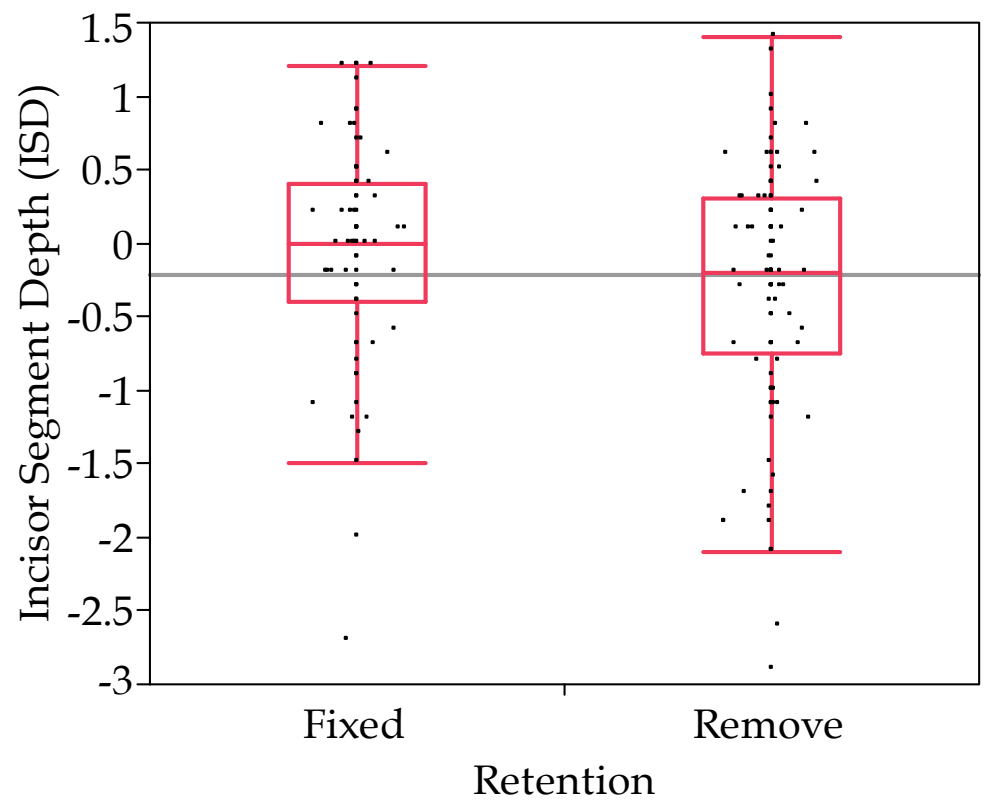

Figure 4-24. Box plot of the amount of relapse in Incisor Segment Depth (ISD) dependent on type of retention.

Significantly more change occurred in ISD in the fixed group after treatment. 


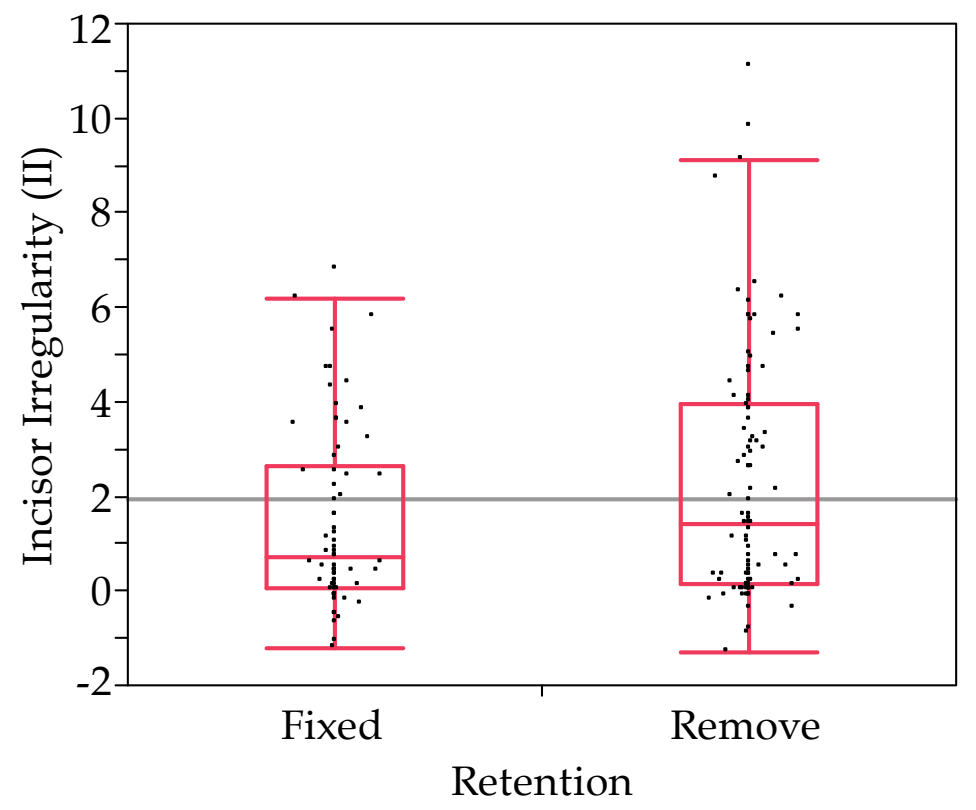

Figure 4-25. Box plot of the amount of relapse in mandibular Incisor Irregularity (II) dependent on type of retention.

That is, the increase in irregularity was statistically greater on average in the removable group $(\bar{x}=2.3 \mathrm{~mm})$ than in the fixed group $(\bar{x}=1.5 \mathrm{~mm})$ during the posttreatment interval. 
more stable (i.e., the mean change was close to zero) compared to greater change in the removable sample. The amount of change in the fixed sample was 1.46 $\mathrm{mm}$ versus $2.30 \mathrm{~mm}$ in the removable group.

Maxillary Intermolar Width (Table C-9) also shows up as a significant variable $(\mathrm{P}=\sim 0.01)$. The sample with fixed retention experienced slight expansion, while cases with just Hawley retainers experienced slight constriction (Figure 4-23). Maxillary Intermolar Width decreased in both groups, but significantly more so in the group with just removable retainers. It is difficult to determine why this effect on maxillary intermolar width shows up between the groups during the recall period. Any attempts at explaining this difference would be purely conjecture.

Incisor segment ratio (Table C-6) shows up as significant variable $(\mathrm{P}=\sim$ 0.01 ). In the sample that received just the Hawley retainers, incisor segment ratio is closer to zero, which indicates that the incisor arch form became more curved and decreased in width after treatment as viewed occlusally (Figure 4-21) more so than in the fixed retention sample.

Incisor Aspect Ratio (Table C-7) reveals only borderline significance $(\mathrm{P}=$ $\sim 0.05)$. Figure 4-22 illustrates that Incisor Aspect Ratio is greater in the fixed group, indicating that incisors remained more upright in cases retained with a fixed retainer as compared to those retained with a Hawley retainer following treatment.

Incisor segment depth (Table $\mathrm{C}-\mathbf{1 3})$ shows up as significant variable $(\mathrm{P}=\sim$ 0.01). Figure 4-24 illustrates that the sample with fixed retention was more stable (i.e., the median hovered on zero) compared to the removable sample.

\section{Extraction Pattern}

Three different extraction patterns proved common enough in the data to allow for statistical analysis (Appendix E). These are (1) nonextraction, (2) extraction of maxillary and mandibular first bicuspids (4-4/4-4), and (3) extraction of maxillary first bicuspids and mandibular second bicuspids (4-4/55). Table E-1 lists the results of two-way ANCOVA tests evaluating whether the amount of relapse was associated with a particular extraction pattern while controlling for the time out of treatment. That is, there is little chance that the occlusion is continuing to change since no one less than 10 years out of treatment was included. Still, partly out of curiosity and partly to confirm the situation, time-out-of-treatment was used as the covariate. In fact, 3 of the 33 variables did show a significant age effect (i.e., ISR, LCW, and ISD). 
Differences among the three extraction patterns (Table E-1) were few. Only 3 variables exhibited significance, namely molar correction $(P=\sim 0.04)$, maxillary intercanine width, which exhibited borderline significance $(P=0.045)$, and anterior segment width $(\mathrm{P}=0.014)$.

Figures 4-26 through 4-28 are graphs of the group differences for these 3 variables. Less molar correction (Figure 4-26) occurred in the nonextraction group than in either of the extraction groups. Extraction of $4-4 / 5-5$ resulted in the largest of molar correction of the three groups.

The greatest change in maxillary intercanine width (Figure 4-27) was in the 4-4/5-5 extraction group, followed by the nonextraction group. The least amount of change occurred in the 4-4/4-4 extraction group.

Figure 4-28 is a graph of the changes in anterior segment width. The least amount of change was in the 4-4/4-4 extraction group, followed by the 4-4/5-5 extraction group, and then the nonextraction group. 


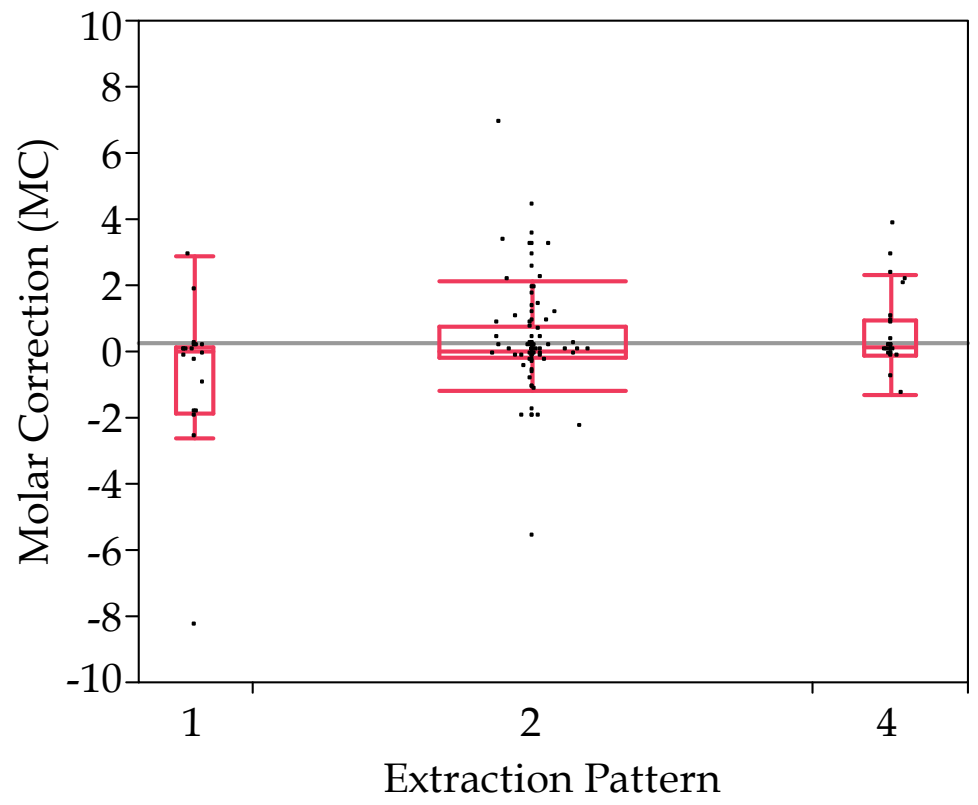

Figure 4-26. Graphic display of Molar Correction (MC) depending on extraction pattern.

Extraction codes are (1) nonextraction, (2) 4-4/4-4, and (4) 4-4/5-5. 


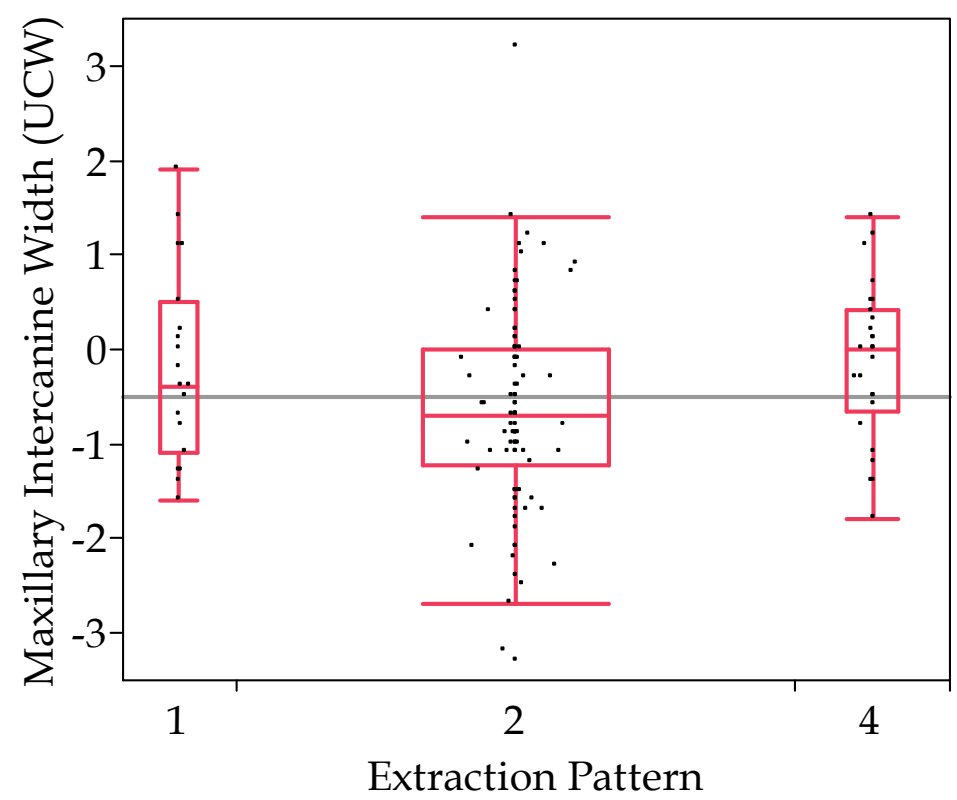

Figure 4-27. Graphic display of Maxillary Intercanine Width (UCW) depending on extraction pattern.

Extraction codes are (1) nonextraction, (2) 4-4/4-4, and (4) 4-4/5-5. 


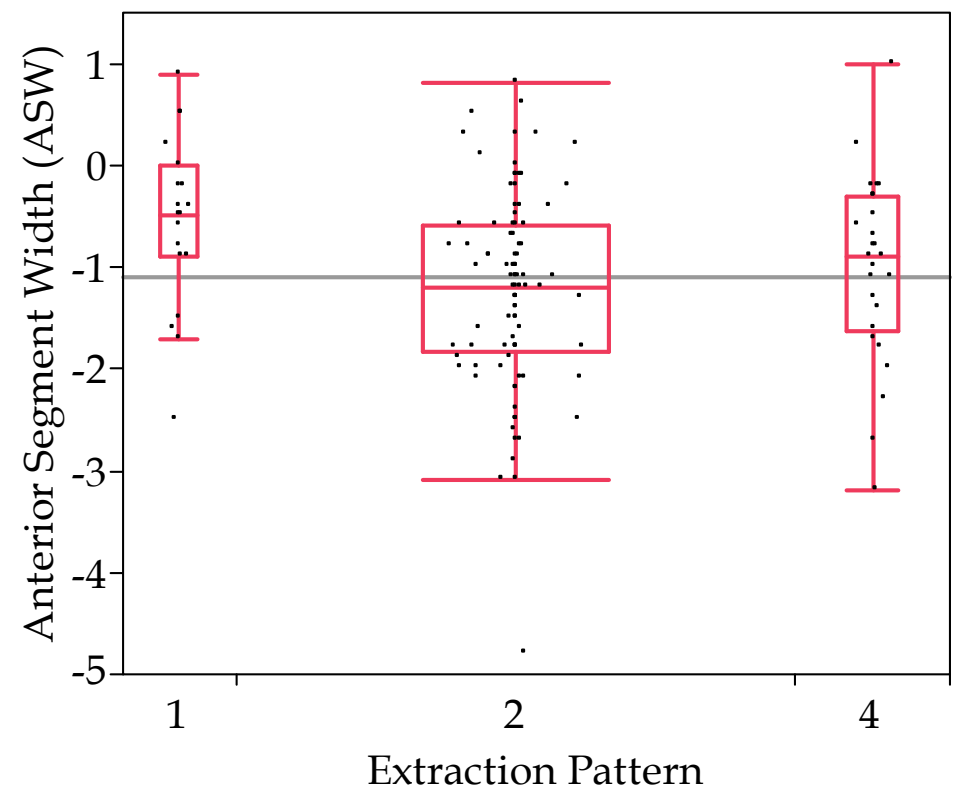

Figure 4-28. Graphic display of Anterior Segment Width (ASW) depending on extraction pattern.

Extraction codes are (1) nonextraction, (2) 4-4/4-4, and (4) 4-4/5-5. 


\section{CHAPTER 5. DISCUSSION}

Relapse - a return towards pretreatment conditions - seems to plague most orthodontic cases to some degree. Similar to the aging process, it is difficult to predict the extent to which an individual case will relapse. On more than one occasion, Gerald Samson (personal communication, February 2009; May, 2010) has said that "As the process of aging progresses, the skin wrinkles and so do teeth." Put another way, "The dentition constantly changes throughout life" (Horowitz and Hixon 1969, p 3). To stop orthodontic relapse is akin to stopping the aging process. Because posttreatment relapse occurs to some degree in all patients, evaluations of the efficacy and rationale of various methods aimed at prevention are valuable.

Lysle Johnston (1993) has asserted that orthodontic treatment may be more stable than had previously been documented. He challenged the specialty to produce other samples and treatments to be subjected to long-term scrutiny. To date, the vast majority of research concerning long-term stability has come from the Department of Orthodontics at the University of Washington (reviewed in Little and Sinclair 2009). The present research intends to provide additional insight into the long-term stability of treated patients. The nature of the present sample (Harris 1985) is that (a) cases are evaluated long-term ( $\geq 10$ years after debanding), (b) cases were treated in private practice, and (c) all cases were treated using standard edgewise mechanics (Sandusky and Gramling 1988).

The two-fold intent of this study was to determine whether the type of retention-either Hawley retainers alone or Hawleys in combination with a fixed lingual retainer-provided greater long-term stability, and to compare the findings of this study to other long-term outcomes. This was a cast analysis where records were gathered before (T1), after (T2), and at least 10 years (T3) following the active phase of orthodontic treatment. A strength of this study is the large sample size of 166 patients, of whom 69 received a maxillary Hawley retainer in combination with either a fixed mandibular canine-to-canine or first premolar to first premolar retainer, and the other 97 received maxillary and mandibular Hawley retainers. Mandibular fixed retainers were removed on average 2 to 3 years after the end of treatment, after which no mandibular retention was used, and the duration of maxillary retainer wear was left up to the discretion of the patient.

Retention is the phase of orthodontic treatment that attempts to keep teeth in their corrected positions following active tooth movement with orthodontic braces (Littlewood et al. 2006). The intent of Hawley retainers is to hold the teeth in their end-of-treatment positions yet allowing them to passively settle into their 
final vertical positions. This phase requires substantial patient compliance. The intent of fixed retention is to maintain an ever-present rigid control, particularly over the lower incisors, that does not depend on patient compliance. The idea is that, if fixed retention remains in place for a couple of years, this allows the periodontal tissues to become reoriented and passive to the new tooth positions and provide stability to incisors during the period of time when most relapse is thought to occur (Reitan 1967; Melrose and Millett 1998; Al Yami et al. 1999). Prolonged fixed retention for a couple of years might also allow fixation of the incisors until issues of the third molars have been addressed (Bergstrom and Jensen 1960; Sheneman 1969).

\section{Sample Considerations}

The cases used for the present study represent a geographically diverse sample consisting of records collected from several orthodontic practices in the United States and Mexico. As noted, all cases were treated with a similar treatment philosophy. Possible shortcomings of this study might be associated with the fact that all of the records were voluntarily submitted by orthodontists in private practice. This collection strategy could allow the orthodontists to cherrypick their cases and submit cases that they perceived to retain more satisfactory results. However, this risk is no greater or worse than any other recall study of this type. We have to rely on the desire and intent of the original data collection of the International Tweed Foundation, which expressly requested doctors to submit cases of all quality to allow for a representative sample of all treatment outcomes. There is no reason to believe this request was not adhered to. In addition, the overall scarcity of 10 year post-treatment records tends to minimize this bias, because in most cases, you are lucky to "get what you can get." An example of this was documented in the sample protocol of two studies out of St. Louis (Paquette et al. 1992; Luppanapornlarp and Johnston 1993). Efforts were made to contact each prospective patient five times. Out of 2,500 prospective patients, only 125 actually participated in the two studies. This recruitment rate of $5 \%$ illustrates the difficulty of obtaining long-term samples.

There also is the insurmountable issue of self-selection. It seems likely that patients with stable (and, thus, esthetic) outcomes would be more willing to participate in a recall study. Conversely, subjects who were non-cooperative patients and/or had unstable outcomes might be less enthusiastic about being reevaluated by their orthodontists since this could leave them open to criticism by the specialist.

Another issue that is difficult to control is the individual orthodontist's commitment to the use of a fixed retainer. Despite the consensus opinion that 
this device enhances stability, it is unlikely that every participating specialist was equally convinced of its efficacy or in what cases it would be most effective. Additionally, there would seem to be a patient component in the decision: Some people would be more concerned about preserving their treatment, while others would be less enthusiastic about dealing with the added efforts to keep the gingiva healthy around the retaining wire.

\section{Discussion Topics}

There are four topics to be addressed here. First, previous research of similar scope will be acknowledged and discussed in reference to present findings. Second, possible explanations for the greater stability found in the present study will be offered. Third, variables that were found to be predictive of the use of fixed retainers are distinguished. Lastly, the clinical relevancy of the present study will be reviewed.

\section{Previous Research and Present Findings}

Previous long-term retention studies are conflicting. Shah (2003) reviewed 29 studies dealing with mandibular incisor stability in an effort to collate current evidence relating to relapse. A wide range was reported between the various studies regarding postretention periods, sample size, inclusion criteria, and amount of relapse. Those studies most closely resembling the present study are reviewed here.

The results of the present study differ from those out of the University of Washington by Little et al. $(1981,1988)$. In summary, these two University of Washington studies showed (a) general relapse of $25 \%$ or greater, (b) the success rate of maintaining satisfactory mandibular incisor alignment (irregularity index of less than $3.5 \mathrm{~mm}$ ) over a prolonged period of time was less than $30 \%$, (c) no significant pretreatment predictors of relapse, (d) relapse is unpredictable, (e) arch width decreased following retention, and (f) lifetime retention is recommended as the only practical safeguard. In the present study, the mean postretention relapse for both retention groups $(2.2 \mathrm{~mm})$, as determined by Little's irregularity index was less than reported by Little et al. $(1981,1988)$, which were $2.9 \mathrm{~mm}$ and $3.6 \mathrm{~mm}$, respectively. Overall success of cases in the present study, as measured by Little's published acceptable irregularity index of less than $3.5 \mathrm{~mm}$ (Little 1975) was 121/166 or $73 \%$, which contrasts previously reported acceptable incidences of 30\% (Little et al. 1981) and 10\% (Little et al. 1988). However, in comparison to the University of Washington studies, we found that arch width (as measured by intermolar width and intercanine width) 
decreased in both arches during the recall period and that degree of Incisor Irregularity at pretreatment was not predictive of instability over the long-term recall period.

Sadowsky and Sakols (1982) evaluated the long-term stability of 96 patients who had been treated between 12 and 35 years previously. Their purpose was to identify variables that fell outside the ideal range of variation at long-term recall. Similar to the present study, this sample also revealed an acceptable amount of incisor stability at the long-term with an average of $2.8 \mathrm{~mm}$ of Incisor Irregularity. However, the authors of this study acknowledged that any possible influence of length of retention was not considered.

Another study by Sadowsky et al. (1994) consisted of a sample of 22 orthodontically treated cases who were evaluated for long-term stability an average of 14.5 years posttreatment. The purpose was to evaluate the long-term stability of cases treated by a single practitioner whose policy was to use longterm fixed retention in the mandibular arch. These patients were retained with a mandibular fixed lingual retainer for an average of 8.4 years, but were without retainers for a minimum of 5 years prior to evaluation. Incisor irregularity was similar to the fixed retention group at the start of treatment $(5.2 \mathrm{~mm}$ versus 5.4 $\mathrm{mm}$ ), at the end of treatment (1.0 $\mathrm{mm}$ versus 0.5$)$, and at the recall stage it was ( $2.4 \mathrm{~mm}$ versus $1.9 \mathrm{~mm}$ ). Sadowsky et al. concluded that less relapse (than other studies have documented) occurred in cases at 5 years postretention when fixed retention was prolonged to age 18. The findings of the present study support this notion; yet also demonstrate an equivalent long-term stability in a similar sample of patients following retention with removable retainers only. Sadowsky et al. acknowledged the importance of the long-term recall period, as well as the shortcomings of their small sample size, but proposed that the increased stability noted in their research may reflect the prolonged mandibular fixed retention. Even though the present study, with a long-term recall period of $\sim 14$ years postretention, and a fixed retainer sample size of 69 cases, demonstrates equivalent statistical stability for mandibular incisors as reported by Sadowsky et al. (1994), it is difficult to attribute this increased degree of stability to prolonged fixed retention, because clinically similar degrees of stability were also demonstrated in a sample of 97 cases retained only by removable retainers.

In a study of 32 patients treated by the same practitioner, who had been out of retention for a minimum of 5 years, Boley et al. (2003) documented acceptable stability of mandibular incisors at an average of 15 years posttreatment (11.7 years postretention). Mean Incisor Irregularity at the longterm recall was $2.6 \mathrm{~mm}$; however, this sample had a much greater degree of Incisor Irregularity at pretreatment than most previous studies $(\bar{x}=8.1 \mathrm{~mm})$. Similar to the present study, there was a much greater percentage of cases with 
acceptable postretention stability at the long-term (80\%) than previously reported (Little et al. 1981, 1988). The authors attributed much of this stability to meeting specific evidence-based treatment objectives during treatment. The present study and others, as reviewed by Boley (2007, p 16), also document that acceptable long-term stability is possible and can be linked to several common characteristics of these "satisfactory stable" samples - including minimal alteration of the mandibular arch form and the retraction and uprighting or maintenance of mandibular incisors in their original position (Boley et al. 2003).

Mandibular Incisor Irregularity often is used as an important indicator of a case's stability (Little 1977), partly because it is a condition obvious to the patient. Figure 5-1 illustrates that group averages do not differ at the pretreatment examination, but at the end of treatment, the fixed group has a significantly greater amount of irregularity. However, by the recall examination, this difference not only disappears, but becomes statistically significant in the other direction, as the removable group now has a statistically significant greater Incisor Irregularity than does the fixed group. This is also evident in the significant difference noted in the rate of change of Incisor Irregularity between the two groups following treatment (Table A-5).

Patients receiving only Hawley removable retainers displayed $\sim 2.5 \mathrm{~mm}$ of Incisor Irregularity, while patients receiving an upper Hawley retainer in combination with a temporary fixed lower retainer displayed only $\sim 1.9 \mathrm{~mm}$ irregularity. This difference between the groups $(\sim 0.6 \mathrm{~mm})$ at the recall examination was found to be statistically significant, but from a clinical standpoint, these differences are minimal, and likely would not be detected by the patient or practitioner. Overall incidence of long-term stability of both groups $(\bar{x} \sim 2.2 \mathrm{~mm}$ ) was greater than previously reported by most studies (Table 5-1).

Other statistically significant changes after treatment occurred in Incisor Segment Ratio and Incisor Aspect Ratio. These two ratios reflect the proclination of the lower incisors. There was a net decrease in Incisor Segment Ratio $(\bar{x}=-0.8$ for the removable group), while there was a net increase $(\bar{x}=0.7$ for the fixed group). There was a greater positive change in Incisor Aspect Ratio in the fixed retention group $(\bar{x}=5.8 \mathrm{~mm})$ compared to the removable group $(\bar{x}=3.1 \mathrm{~mm})$. Considered together, these changes suggest that the lower incisors tipped forward more in the removable group than in the fixed group. This does not necessarily mean that alignment deteriorated, only that there was a greater proclination. There was also a greater decrease in maxillary intermolar width during this period in the removable group than in the fixed group. It is unclear why this might be. 


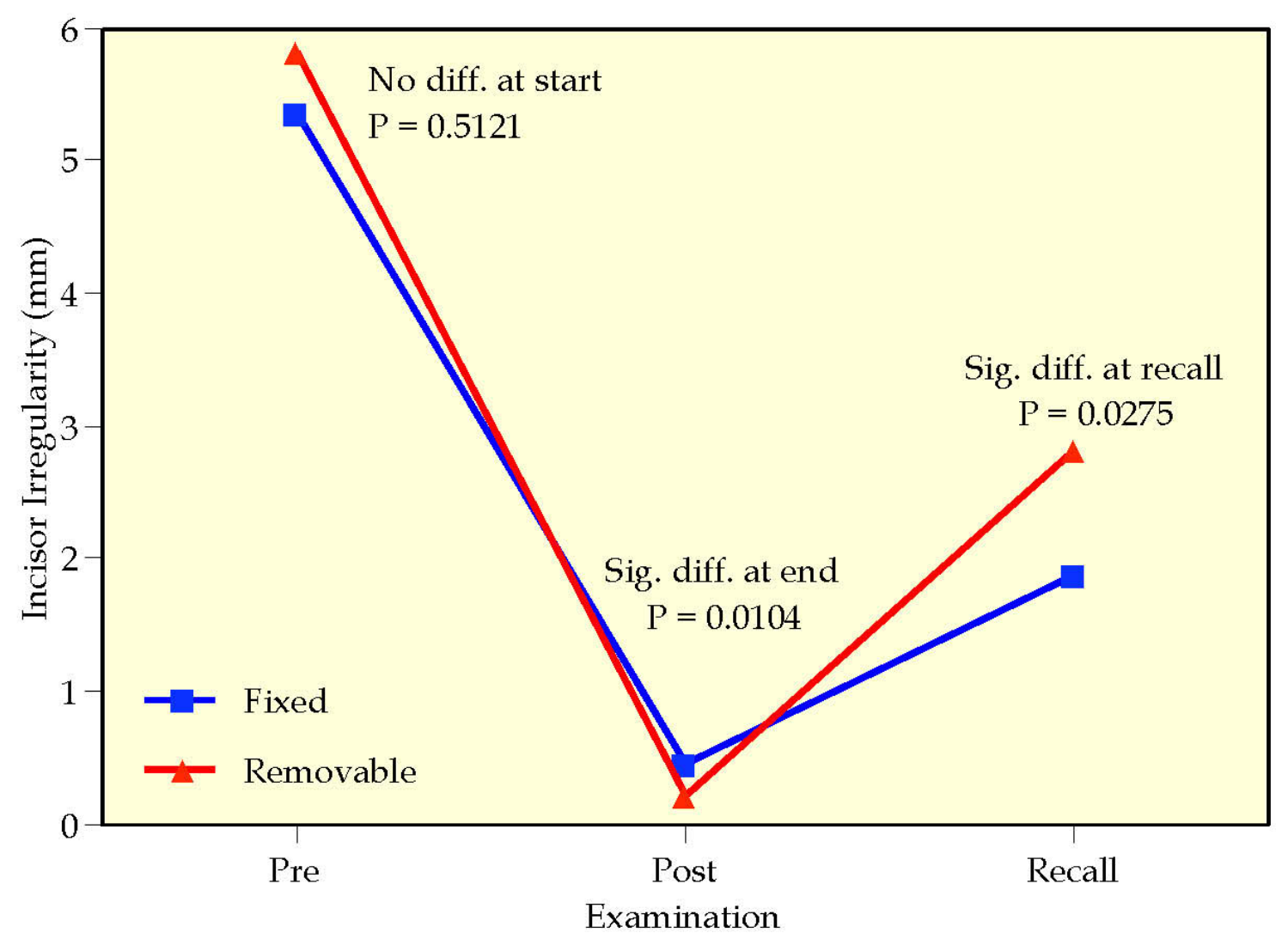

Figure 5-1. Plot of average Incisor Irregularity, by group, at the three examinations.

Though statistically significant, the fixed retention appliance only enhances stability by an average of $0.6 \mathrm{~mm}$ at the long-term recall examination. 
Table 5-1. Summary of prior studies that investigated the long-term stability of orthodontic treatment for mandibular Incisor Irregularity (II).

\begin{tabular}{|c|c|c|c|c|c|c|c|c|c|c|c|}
\hline Research & $(1)$ & $(2)$ & (3) & $(4)$ & (5) & (6) & $(7)$ & $(8)$ & $(9)$ & $(10)$ & $(11)$ \\
\hline Year & 1981 & 1982 & 1987 & 1988 & 1989 & 1992 & 1993 & 1994 & 1996 & 2003 & 2010 \\
\hline Sample Size (n) & 65 & 96 & 28 & 31 & 30 & 63 & 62 & 22 & 78 & 32 & 166 \\
\hline Mean Postretention & 13 & $20 \#$ & 8 & 10 & 14 & 15 & 15 & 6.3 & 14 & 12 & 13.5 \\
\hline Range Postretention & $10-24$ & $12-35^{\#}$ & $3-$ & na & $10-22$ & $9-20$ & $11-23$ & $5-17$ & $9-33$ & $5-22$ & $8-44$ \\
\hline \multicolumn{12}{|l|}{ Start of Treatment } \\
\hline Mean Age (yrs) & $13 \dagger$ & na & 13 & $13^{\dagger}$ & $13^{\dagger}$ & 13 & 13 & 11 & 11 & 13 & $13^{\dagger}$ \\
\hline Mean II (mm) & 7.3 & 8.2 & 2.9 & 7.4 & 2.5 & 5.8 & 5.1 & 5.2 & 4.1 & 8.1 & 5.7 \\
\hline SD II (mm) & 4.3 & 3.2 & 1.4 & na & na & na & na & 4.2 & 3.0 & na & 3.9 \\
\hline \multicolumn{12}{|l|}{ End of Treatment } \\
\hline Mean Age (yrs) & $15^{\dagger}$ & na & 15 & $16^{\dagger}$ & $15^{\dagger}$ & 14 & 15 & na & na & $15.5^{\dagger}$ & $15.8^{\dagger}$ \\
\hline Mean II (mm) & 1.7 & na & 1.0 & 1.7 & 1.5 & 0.6 & 0.6 & 1.0 & 1.0 & 1.8 & 0.4 \\
\hline SD II (mm) & 0.6 & na & 0.5 & na & na & na & na & 0.8 & 0.8 & 0.9 & 0.6 \\
\hline \multicolumn{12}{|l|}{ Recall Examination } \\
\hline Mean Age (yrs) & $30^{\dagger}$ & na & 27 & $30^{\dagger}$ & $32^{\dagger}$ & 29 & 30 & na & 31 & $31.6^{\dagger}$ & $32.2^{\dagger}$ \\
\hline Mean II (mm) & 4.6 & 2.8 & 2.2 & 5.3 & 3.8 & 3.2 & 3.5 & 2.4 & 3.8 & 2.6 & 2.2 \\
\hline SD II (mm) & 1.9 & 2.7 & 1.7 & na & na & na & na & 1.7 & 2.0 & 1.2 & 2.3 \\
\hline
\end{tabular}

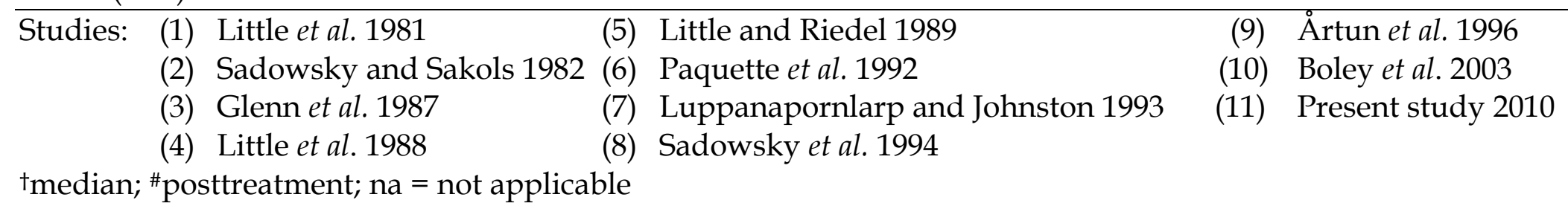


Based on the present results, the use of temporary fixed retention $(\sim 2.5$ years) following orthodontic treatment does allow for slightly less changes in Incisor Irregularity over the long-term. However, does this clinically inconsequential amount of change over the long-term (beyond 10 years) warrant the added time, expense, and hassle of placing a fixed retainer for such a transient time? We propose that if the time and effort is spent to use fixed retention at the end of treatment, plans for its indefinite use should be considered.

\section{Explanation for Stability}

Two important tenets of Tweed treatment mechanics (Sandusky and Gramling 1988) are to minimize mandibular canine expansion during treatment and to upright mandibular incisors over basal bone. In the majority of cases examined in the present study, both of these treatment goals were achieved. Expansion of the mandibular intercanine width was minimized during treatment. The average mandibular intercanine width for both retention groups prior to treatment was $24.5 \mathrm{~mm}$. Following treatment, this dimension increased to $25.7 \mathrm{~mm}$, resulting in an average in-treatment increase of just over $1.0 \mathrm{~mm}$.

Two cephalometric measurements, FMIA and IMPA, are used to evaluate the position of the mandibular incisors relative to the basal bone. The average FMIA for both retention groups prior to treatment was $60.2^{\circ}$, and $62.5^{\circ}$ at posttreatment, for an average intreatment change of $2.3^{\circ}$. The average IMPA for both retention groups prior to treatment was $93.0^{\circ}$ and posttreatment was $91.1^{\circ}$, resulting in an average in-treatment reduction of $1.9^{\circ}$.

This is compelling evidence that the orthodontists in the present study recognized and attempted to (1) minimize the expansion of the dental arches because expansion of lower intercanine width is the most predictable of all orthodontic relapse (McCauley 1944; Tweed 1944; 1945; Riedel 1960; Steadman 1961; Lundström 1968; Bishara et al. 1973; Herberger 1981; Glenn et al. 1987; Bishara et al. 1989; Vaden, Harris, and Gardner 1997; Blake and Bibby 1998; Boley et al. 2003) and (2) maintain or upright the lower incisors because advancing the lower incisors is unstable and may seriously compromise posttreatment stability of the lower incisors (Lundström 1925; Tweed 1944; 1945; Riedel 1976; Shields et al. 1985; Houston and Edler 1990; Boley et al. 2003).

The important influence that these two treatment goals have on the longterm stability of orthodontically treated cases is well documented by research referenced previously in the current study and in the final conclusions of Blake and Bibby (1998) in their review of the literature regarding retention and 
stability. The most stable position of the lower incisor is its pretreatment position and the patient's pretreatment lower arch form should be maintained during orthodontic treatment as much as possible (Blake and Bibby 1998). It seems that the close adherence to these two principles during treatment of both samples in the present study significantly influenced the acceptable long-term incisor stability documented in these cases.

\section{Predictors for Fixed Appliances}

Since barely half of the 166 cases reviewed here $(42 \% ; 69 / 166)$ were retained with a fixed lingual retainer, there must have been appreciable differential diagnoses of the cases in terms of who would benefit from this appliance in addition to Hawley retainers. A multivariate statistical method was used in an effort to tease-out those dental and skeletal features that influenced the orthodontist's decision to use fixed retention. The assumption was that those variables that were systematically different between the groups would be those that actually influenced the orthodontist's decision process.

Stepwise discriminant functions analysis was used. All of the cast and cephalometric variables were input for the start of treatment to see whether the orthodontist might have anticipated the use of a fixed retainer based on the initial presentation of the malocclusion. Despite having a large battery of variables, only total chin and overbite were significantly different between the types of retention. Notably, this does not necessarily mean that these variables were used by the orthodontist to determine who would receive a fixed retainer because these same dimensions persisted as significant predictors at the end of treatment, which probably was when the orthodontist actually settled on the type of retention to be used.

Discriminant analysis then was used to evaluate which variables measured at the end of treatment might be predictive of the type of retention prescribed. At the end of treatment, the most-distinguishing variables associated with the use of fixed retention are the following: (1) greater incisor depth, (2) greater overbite, (3) greater total chin dimension, and (4) greater Incisor Irregularity. It is supposed that these end-of-treatment conditions colored the orthodontist's decision of whether fixed retention would be beneficial.

The amount of incisor uprighting or lack thereof seems to have influenced the decision about which type of retention was used. Following treatment, mean incisor depth was greater in the fixed retention group ( $24.0 \mathrm{~mm}$ versus $20.0 \mathrm{~mm}$ ). IMPA was less in the removable group $\left(90.6^{\circ}\right.$ versus $\left.91.6^{\circ}\right)$, and FMIA was slightly less in the fixed retention group $\left(62.4^{\circ}\right.$ versus $\left.62.6^{\circ}\right)$. Taken together, 
these variables indicate more anteriorly positioned mandibular incisors and increased the likelihood that a case would be put in fixed retention.

Overbite shows up at both the pretreatment and posttreatment examination as being significantly greater in the fixed retention group than the removable group. The clinical reason for this is not clear, but two possibilities are (1) that greater overbite is associated with greater Incisor Irregularity and/or (2) that greater overbite is associated with (a consequence of) incomplete orthopedic correction of the intermaxillary skeletal relationships, that is a failure of incisor coupling. Either case might be seen as a risk factor for relapse-thus the use of a fixed retainer in hopes of enhancing the chances for stability. It is difficult to determine what significance, if any, an increased overbite might have on the stability of lower incisors, although Sadowsky (1982) demonstrated that in a sample of 96 treated patients, more severe mandibular anterior crowding $(>3.0$ $\mathrm{mm}$ ) usually occurred together with an excessive overjet and overbite at longterm recall. In another study by Kahl-Nieke et al. (1995), 226 cases with various Angle's classifications of malocclusion were examined to document postretention changes and to distinguish factors that may play a role as predictors for long-term prognosis. The authors also found an association between increased pretreatment overbite and increased amounts of postretention incisor crowding and Incisor Irregularity.

A greater total chin dimension persisted from the pretreatment evaluation to the posttreatment evaluation in the sample receiving fixed retainers. Indeed, this was the single most predictive measure of who received a fixed retainer. It is interesting that Merrifield (1966) recognized this as a useful clinical measure. As defined by Merrifield (1966), total chin is a millimetric measurement including the bony chin lying anterior to the NB line and measured to soft tissue Pogonion (Figure 3-22).

Merrifield acknowledged that some investigators have placed great significance on the bony chin, but Merrifield himself made no association between the total chin measurement and the stability of lower incisors. Merrifield (1966) found the overall evaluation of the total chin to be more important in the study of facial esthetics and profile evaluation, and he attributed changes in total chin thickness to the individual's growth (particularly of the mandible) and developmental changes. Stoner and Lindquist (1956) proposed that the lower incisors have a definite relationship to facial esthetics, though possibly indirectly. Holdaway (1956) emphasized the important contribution the bony chin has on facial esthetics, and further indicated that a recontouring of Point $B$ by lingual movement of the lower incisors increased the effective bony chin and was accompanied by improvement in facial balance. 
It is difficult to draw firm conclusions as to why total chin shows up as a statistically significant predictive variable at the pretreatment stage and again at posttreatment based on the results of the present study. The greater total chin dimension that persisted from the pretreatment stage to posttreatment stage in the fixed retention group was associated with more anteriorly positioned mandibular incisors at both treatment stages, as evidenced by greater IMPA and incisor depth.

Many clinicians today consider fixed retention in the treatment planning phase when severe pretreatment Incisor Irregularity is present. In the present study, there was no statistical difference in Incisor Irregularity prior to treatment (5.4 $\mathrm{mm}$ in the fixed group versus $5.9 \mathrm{~mm}$ in the removable group). However, by the end of treatment, Incisor Irregularity was significantly greater in the group ( $0.5 \mathrm{~mm}$ versus $0.2 \mathrm{~mm}$ ) in which a fixed retainer was used. Greater Incisor Irregularity at the end of treatment seems to have added to a sense of incomplete orthodontic correction and greater risk for future instability.

A deeper Curve of Spee persisted in the fixed retention group following treatment $(0.8 \mathrm{~mm}$ versus $0.4 \mathrm{~mm})$, albeit small, and this was identified as a significant predictor in the multivariate statistical analysis. Incomplete leveling of the Curve of Spee seems to have influenced the decision in favor of using fixed retention. Yet when evaluating the amount of change in the Curve of Spee during the recall period, the removable group displayed a slightly greater relapse ( $0.6 \mathrm{~mm}$ versus $0.4 \mathrm{~mm}$ ), revealing that fixed incisor retention added little to maintaining the Curve of Spee correction. Along this same line, lack of molar correction during treatment (where a Class I molar relationship was not fully

achieved) was greater in the fixed retention group ( $1.5 \mathrm{~mm}$ versus $1.0 \mathrm{~mm})$. This difference was statistically significant when evaluated univariately (Table A-2), and could have been the result of the decreased mandibular incisor uprighting also seen in this group.

Considered together, there was a deeper Curve of Spee and greater molar relationship during treatment in the removable group than in the fixed retention group. This suggests that fixed retention could have been considered following treatment if there was a sense of incomplete correction or an inability to achieve the desired degree of orthodontic correction.

\section{Clinical Relevance}

Our perception is that the use of temporary fixed retention following orthodontic treatment has largely been abandoned. This is predominately because of concerns over maintenance responsibility, periodontal health, caries 
and the hassle associated with placing the appliance following treatment. Results of the present study document that long-term stability is clinically no greater in patients retained by fixed retention for a short time following active treatment (ca. 2 years) than patients receiving removable retention alone. Therefore, when weighing the advantages versus disadvantages of placing a fixed lower retainer (time, money, trouble, maintenance, etc.), it seems that the fixed retainer has little clinical advantage if only placed temporarily, and that if a fixed retainer is to be used, it should be planned to remain in place indefinitely. Furthermore, studies have established that relapse occurs to some degree in combination with the normal aging process in most every patient, and that retention to some degree is needed following orthodontic treatment (Sillman 1964; Lundström 1969; Little et al. 1981, 1988; Behrents 1985a, 1985b, 1989; Al Yami 1999; Harris et al. 1999; Richardson 1999; Stephens et al. 2005). What continues to be debated is the broad question of why this relapse occurs and to what extent it will occur in individual patients.

Currently, it seems the majority of fixed retainers, when placed, are anticipated to be left indefinitely. Littlewood et al. (2006) concluded that there is insufficient research on which to base clinical judgments. From their conclusions and based on the research presented here, it can be inferred that no current mode of retention is superior to another.

One caveat to this conclusion is the individualized variability in growth changes that takes place in postorthodontic patients. Growth studies by Behrents (1985, 1986a, 1986b), Nanda $(1971,1990)$ and Harris et al. $(1994,1997)$ have documented the variability in craniofacial growth changes among individuals. These changes do not stop in young adulthood, but continue throughout life. It was probably on this premise that Riedel (1960) contended that all patients must be thoroughly evaluated to determine what type, if any, and for how long retention procedures should be undertaken. Interestingly, Nanda and Nanda (1992) proposed that retention devices should be differentially selected on the basis of dentofacial morphology and the anticipated magnitude and directions of growth instead of simply using the clinician's retainer of choice for all cases. Nanda and Nanda (1992) stress the importance of maintaining "active" retention through the pubertal growth spurt until maxillomandibular growth is completed, and they assert that the clinician has to be alert to the growth pattern of each individual patient. Specific examples of customized retention cited by the authors include a) continued use of a high-pull headgear in long-face syndrome patients during the retention phase, $b$ ) addition of an anterior biteplane to the retaining device in short-face syndrome patients, and c) longer retention periods for patients experiencing a delayed pubertal growth spurt, i.e. patients with skeletal deep bite. 
Horowitz and Hixon (1969, p 4) described physiologic recovery as, "The type of posttreatment changes that represent a rebound or reversion toward the original malocclusion." Based on this premise, it could be assumed that cases that exhibit greater severity of pretreatment malocclusion and undergo the most in-treatment changes could be expected to exhibit a greater degree of posttreatment relapse. This was demonstrated in a recent study at the University of Washington involving 86 patients was undertaken to identify factors associated with stability (Ormiston et al. 2005). The authors found the initial severity of malocclusion to be negatively correlated with posttreatment stability. They concluded that patients with more severe index scores before treatment tended to be less stable.

This common trend also was evident in the present study (Figure 5-2). There is a significant statistical association between the amount of change during treatment and the amount of relapse $(\mathrm{P}=0.0084)$. Prediction of the amount of relapse from the treatment change takes the form of $Y=1.26-0.13(X)$ for the 166 cases in this study, meaning that a $1.0 \mathrm{~mm}$ change in Incisor Irregularity is associated with $0.13 \mathrm{~mm}$ of relapse.

The premise of posttreatment orthodontic retention is based on histological research that shows that it takes time for the surrounding periodontal tissues to reorient themselves following tooth movement (Reitan 1959, 1967; Rygh et al. 1986). Reorganization of the periodontal ligament is estimated to occur over a 3 to 4 month period of time following treatment (Reitan 1959, 1967, 1969; Melrose and Millett 1998), whereas the gingival collagen-fiber network typically takes 4 to 6 months to be remodeled, and the elastic supracrestal fibers have been shown to remain deviated for more than 232 days (Reitan 1967; Melrose and Millett 1998). According to Paulson (1992), bonded retainers should be left in place for only 3 to 4 years, except in cases involving adolescent patients still undergoing their adolescent growth spurt, because any longer than 4 years exceeds the recommendation from Reitan's work $(1959,1969)$ in regards to the time necessary for elastic fiber reorganization. The results of the present study do not support this. In the sample of 166 cases at an average of 15 years postretention, there existed only a $0.6 \mathrm{~mm}$ difference in mandibular Incisor Irregularity between those cases receiving mandibular fixed retention for approximately 2-3 years and those receiving only removable Hawley retainers for the same period of time.

Despite no apparent long-term benefit, some orthodontists might consider that the use of temporary fixed retention affords other benefits. Typically, most comprehensive treatment is completed around the age of 16. By instituting fixed retention for a couple of years at this age, the patient is carried through 


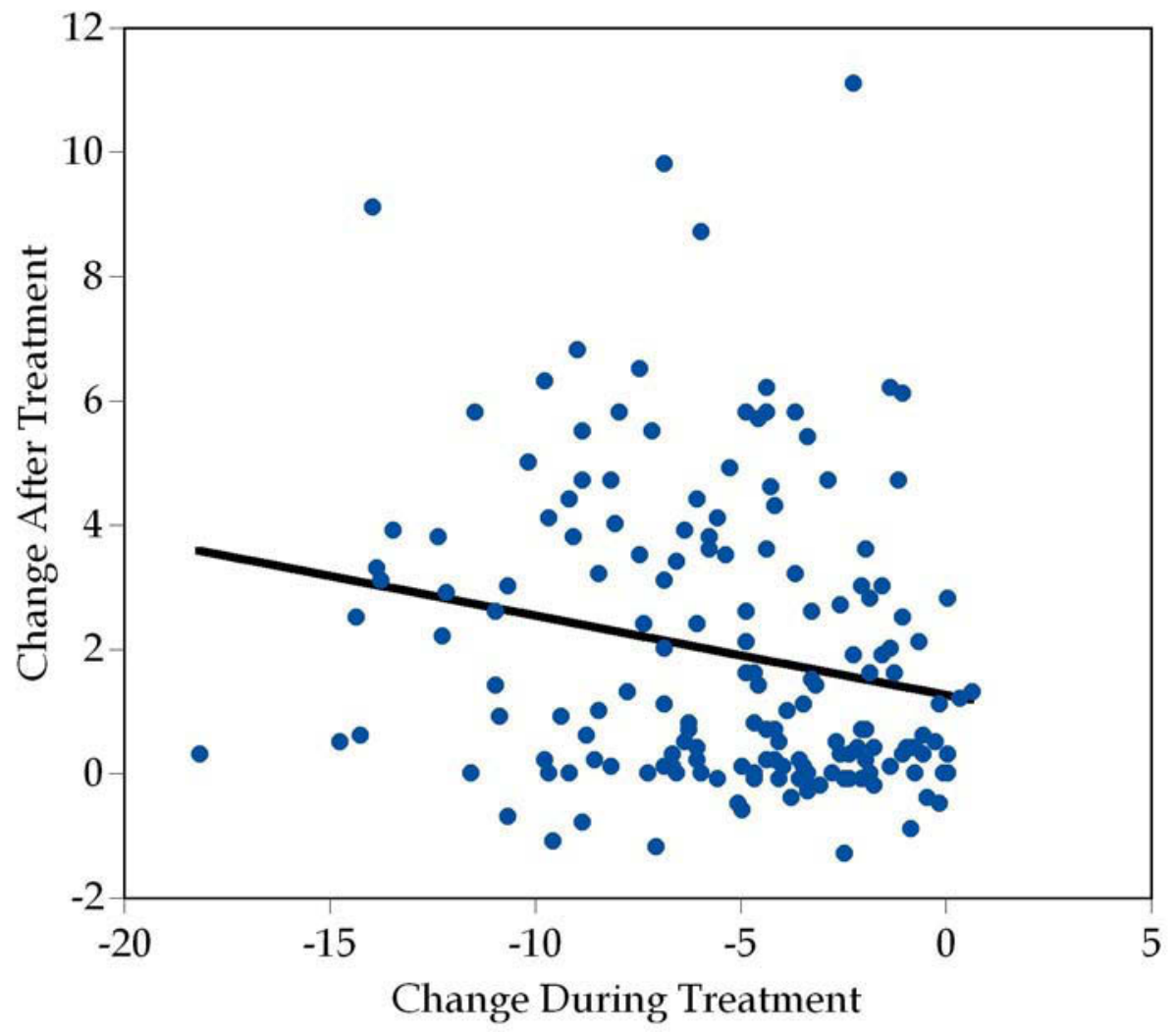

Figure 5-2. Scatter-plot graph reflecting the association for Incisor Irregularity between the amount of relapse following treatment and the amount of change during treatment. 
adolescence, into young adult status, and into the early years of college when expectations of judicious patient compliance can be considered questionable at best. Fixed retention during this period might also allow for the controversial issue of the third molars to be addressed. Finally, an additional few years of fixed retention might provide adequate time for a sense of disconnect from the orthodontic practice. As time goes by, changes in the dentition might be perceived to be the result of aging or the lack of retainer compliance, and not a result of the orthodontic treatment.

Judgments concerning posttreatment retention and stability have become more of a practice management issue than a clinical issue. If the incisors can be mechanically retained by some permanent form of retention, then there seems less of a chance for future problems related to stability, no compliance issues with patients, and a "stick it and forget it" perspective. These retainers must be periodically maintained and monitored for signs of distortion, breakage, or adverse effects on the periodontium. Where the responsibility lies for this maintenance must be clear from the beginning of a patient's treatment. If removable forms of retention are relied on to maintain lifelong stability, the patient needs to assume liability for his or her occlusal status by faithful retainer - wear as instructed on an individual basis. In either case, obtaining documentation of informed consent from the patient is critical from both a standpoint of patient awareness and that of legal responsibility. Written communication that describes where responsibilities lie regarding stability and the prevention of unpredictable changes that can occur as a result of the normal aging process is paramount to prevent future misunderstandings or litigation that might arise as a result of poor communication.

The results of the present research indicate that, contrary to previous reports, overall stability may be better than originally documented. However, similar to previous reports, there is no doubt that, to guarantee stability, one of two things must occur. Either lifetime fixed retention must be planned for the patient, or the patient must acknowledge that he or she must be compliant with long-term removable retainer wear. Based on the results of the present study, we conclude that even though there was a slight statistical benefit, there is no clinical long-term occlusal benefit gained from temporary fixed retention. Because no form of retention has been found to be superior to another (Littlewood et al. 2006), patients who maintain life long compliance with their removable retainers should expect the same degree of stability as those patients who rely on permanent fixed retainers.

In anticipation of the occlusal instability that can be expected to occur in treated and nontreated patients alike in response to the normal aging process, the questions that remain are "who should be responsible for posttreatment 
stability?" and "for how long?" "Should patients at some point expect to own the obligation to their own health, or is this just a part of the ongoing professional duty of dental professionals, such as orthodontists, general dentists, and hygienists, to continue to be liable for?" These likely are questions that cannot be answered by research such as this. These answers will likely come from the obligations felt by individual practitioners toward their patients.

Because the dentition continues to change with age, regardless of orthodontic treatment, Horowitz and Hixon (1969) recommend that there are two important responsibilities placed upon the orthodontist: first, to maintain the highest standards of excellence in rendering treatment as reflected in the conclusions noted by Blake and Bibby (1998) and, second, to record carefully changes in the dentition as they are periodically observed in the years following treatment. 


\section{CHAPTER 6. SUMMARY AND CONCLUSIONS}

Postretention results are the method used to measure the success of orthodontic diagnosis and treatment. Tirk (1965, p 165) said, "The result of orthodontic therapy - good, bad, or indifferent - is only evident many years out of retention."

This longitudinal cephalometric and cast analysis examined the diagnostic records of cases treated comprehensively by several experienced orthodontists who use standard Edgewise mechanics. Of the 166 cases, 69 patients $(23$ males; 46 females) had been placed in a fixed mandibular canine-to-canine retainer and a maxillary removable retainer (traditional Hawley-type) at the end of active treatment for 2 to 3 years. The other 97 patients (20 males; 77 females) were placed in maxillary and mandibular removable retainers (traditional Hawleytype) for the same period of time. The two-fold purpose of this study was to: a) identify whether the type of retention - either Hawley retainers or Hawleys in combination with a fixed lingual retainer - provided greater long-term stability and b) review and document the long-term posttreatment changes of orthodontic cases. Treatment records were made at the start of treatment, at the end of active treatment, and at a long-term recall examination. The average long-term recall period for these patients is 16 years out of the active phase of treatment (13.5 years postretention).

Major findings were:

- Temporary use ( 2.5 years) of a mandibular fixed retainer following comprehensive orthodontic treatment appears to add little to long-term stability; therefore, if fixed retention is considered for retention, it should remain indefinitely to ensure stability.

- The amount of relapse seen in both groups was quite small, and the clinical difference between groups at the long term (average $\sim 0.6 \mathrm{~mm}$ ) was even smaller.

- Mandibular intercanine width was slightly expanded during treatment, but by the long-term recall examination, this dimension had returned to its pretreatment value.

- The maxillary and mandibular arches both became slightly narrower after treatment as measured by intermolar width and intercanine width.

- Overjet and overbite both increased after treatment. 
- About two-thirds (64\%) of the correction of mandibular Incisor Irregularity was maintained over the long-term period. The mandibular Incisor Irregularity at the recall examination was less than $3.5 \mathrm{~mm}$ in $73 \%$ of cases, which is the limit suggested by Little (1975) as being clinically acceptable.

- There is a significant statistical association between the amount of change during treatment and the amount of relapse. Prediction of the amount of relapse from the treatment change takes the form of $Y=1.26-0.13(X)$ for the 166 cases in this study, meaning that a $1.0 \mathrm{~mm}$ change in Incisor Irregularity is associated with $0.13 \mathrm{~mm}$ of relapse.

- These results ( $2.2 \mathrm{~mm}$ Incisor Irregularity at long-term recall) seem more stable than those reported in most long-term recall studies.

It is likely that posttreatment changes and retention protocols will continue to cause discussion among the orthodontic community. Both groups of patients in this study treated under the Tweed guidelines remained extremely stable regardless of which retention protocol was used. However, patients must be conditioned to the potential occlusal changes that could occur, and it must be impressed on them the importance of their diligence in maintaining the stability of their dentition as they age. Further research should be done to determine if these same results could be expected for other treatment philosophies that do not observe such strict treatment parameters, i.e., upright mandibular incisors, minimal if any lower intercanine expansion, and the like. 


\section{LIST OF REFERENCES}

Al Yami EA, Kuijpers-Jagtman AM, van't Hof MA. Stability of orthodontic treatment outcome: follow-up until 10 years postretention. Am J Orthod Dentofacial Orthop 1999;115:300-4.

Alexander RG. The 20 principles of the Alexander Discipline. Chicago: Quintessence Publishing Company; 2008.

Anderson AB. A survey of treatment characteristics in a university based graduate orthodontic program. M.D.S. thesis, University of Tennessee, Memphis, 2010.

Andrews LF. The six keys to normal occlusion. Am J Orthod 1972;62:296-309.

Angle EH. Classification of malocclusion. Dent Cosmos 1899;41:248-64, 350-7.

Angle EH. Treatment of malocclusion of the teeth, 7th ed. Philadelphia: The SS White Dental Manufacturing Company; 1907.

Angle EH. Treatment of malocclusion of the teeth and fracture of the maxillae, 6th ed. Philadelphia: The SS White Dental Manufacturing Company; 1900.

Årtun J. Caries and periodontal reactions associated with long-term use of different types of bonded lingual retainers. Am J Orthod 1984;86:112-8.

Årtun J, Garol JD, Little RM. Long-term stability of mandibular incisors following successful treatment of Class II, Division 1, malocclusions. Angle Orthod 1996;66:229-38.

Årtun J, Spadafora AT, Shipiro PA. A 3-year follow-up study of various types of orthodontic canine-to-canine retainers. Eur J Orthod 1997;19:501-9.

Årtun J, Spadafora AT, Shipiro PA, McNeill RW, Chapko MK. Hygeine status associated with different types of bonded, orthodontic canine-to-canine retainers: a clinical trial. J Clin Periodontol 1987;14:89-94.

Atack N, Harradine N, Sandy JR, Ireland AJ. Which way forward? Fixed or removable lower retainers. Angle Orthod 2007;77:954-9.

Ballard ML. Asymmetry in tooth size: a factor in the etiology, diagnosis and treatment of malocclusion. Angle Orthod 1944;14:67-70. 
Baume LJ, Horowitz HS, Summers CJ, Dirks OB, Brown WAB, Carlos JP, Cohen

LK, Freer TJ, Harvold EP, Moorrees CFA, Salzmann JA, Schmuth G, Solow

B, Taatz J. A method for measuring occlusal traits. Int Dent J 1973;23:530-7.

Bearn DR. Bonded orthodontic retainers: a review. Am J Orthod Dentofacial Orthop 1995;108:207-13.

Begg PR, Kesling PC. Begg orthodontic theory and technique, 3rd ed. Philadelphia: WB Saunders Company; 1971.

Behrents RG. An atlas of growth in the aging craniofacial skeleton. Monograph 18, Craniofacial Growth Series. Ann Arbor: Center for Human Growth and Development, University of Michigan, 1985b.

Behrents RG. Adult craniofacial growth. In: Enlow DH, Hans MG. Facial growth, 2nd ed. Ann Arbor: Needham Press; 2008. p. 291-306.

Behrents RG. The consequences of adult craniofacial growth. Monograph 22, Craniofacial Growth Series. Ann Arbor: Center for Human Growth and Development, University of Michigan, 1989.

Behrents RG. Growth in the aging craniofacial skeleton. Monograph 17, Craniofacial Growth Series. Ann Arbor: Center for Human Growth and Development, University of Michigan, 1985a.

Behrents RG, White L. JCO interviews Dr. Rolf Behrents on adult craniofacial growth. J Clin Orthod 1986;20:842-7.

Bergstrom K, Jensen R. The significance of third molars in the aetiology of crowding. Trans Eur Orthod Soc 1960;11:90-1.

Bishara SE, Chadra JM, Potter RE. Stability of intercanine width, overbite and overjet correction. Am J Orthod 1973;63:588-95.

Bishara SE, Jakobsen JR, Treder JE, Stasi MJ. Changes in the maxillary and mandibular tooth size-arch length relationship from early adolescence to early adulthood. Am J Orthod Dentofacial Orthop 1989;95:46-59.

Bishara SE, Jakobsen JR, Treder JE, Nowak A. Arch length changes from 6 weeks to 45 years. Angle Orthod 1998;68:69-74.

Björk A. Prediction of mandibular growth rotation. Am J Orthod 1969;55:585-99. 
Black GV. Operative dentistry, Vol. 4. Chicago: Medico-Dental Publishing Company; 1936. p. 9-32.

Blair RN, Harris EF. Sex differences in esthetic treatment needs of adolescent orthodontic patients. J Dent Res 2008; Abstract 0332.

Blake M, Bibby K. Retention and stability: a review of the literature. Am J Orthod Dentofacial Orthop 1998;114:299-306.

Boese LR. Fiberotomy and reproximation without lower retention-nine years in retrospect: Parts I and II. Angle Orthod 1980;50:88-97, 169-78.

Boley JC. Review of the literature on post retention stability of mandibular incisors. CH Tweed Int Found Orthod Res Edu 2007;6:13-6.

Boley JC, Mark JA, Sachdeva RCL, Buschang PH. Long-term stability of Class I premolar extraction treatment. Am J Orthod Dentofacial Orthop 2003;124:277-87.

Bolton WA. The clinical application of a tooth size analysis. Am J Orthod 1962;48:504-29.

Bolton WA. Disharmony in tooth size and its relation to the analysis and treatment of malocclusion. Angle Orthod 1958;28:113-30.

Booth FA, Edelman JM, Proffit WR. Twenty-year follow-up of patients with permanently bonded mandibular canine-to-canine retainers. Am J Orthod Dentofacial Orthop 2008;133:70-6.

Bresonis WL, Grewe JM. Treatment and posttreatment changes in orthodontic cases: overbite and overjet. Angle Orthod 1974;44:295-9.

Broadbent BH. The influence of the third molars on the alignment of the teeth. Am J Orthod Oral Surg 1943;29:312-30.

Brodie AG. Does scientific investigation support the extraction of teeth in orthodontic therapy. Am J Orthod Oral Surg 1944;30:445-60.

Burke SP, Silveira AM, Goldsmith LJ, Yancey JM, Van Stewart A, Scarfe WC. A meta-analysis of mandibular intercanine width in treatment and postretention. Angle Orthod 1998;68:53-60. 
Case CS. Techniques and principles of dental orthopedia. Chicago: CS Case Company; 1921.

Case CS, Cryer MH, Dewey M. The extraction debate of 1911. Am J Orthod 1964;50:656-8, 660-91, 751-68, 843-56, 900-12.

Cerny R, Lloyd D. Dentists' opinions on orthodontic retention appliances. J Clin Orthod 2008;42:415-9.

Cooley WW, Lohnes PR. Multivariate data analysis. New York: Wiley; 1971.

Dahl EH, Zachrisson BU. Long-term experience with direct bonded lingual retainers. J Clin Orthod 1991;25:619-32.

Dawson P. Functional occlusion from TMJ to smile design. St. Louis: CV Mosby; 2007.

De Koch WH. Dental arch depth and width studied continually from 18 years of age to adulthood. Am J Orthod 1972;62:56-66.

De La Cruz A, Sampson P, Little RM, Årtun J, Shapiro PA. Long-term changes in arch form after orthodontic treatment and retention. Am J Orthod Dentofacial Orthop 1995;107:518-30.

Dewey M. Practical orthodontia. St. Louis: CV Mosby Company; 1914.

Dewey M, Anderson GM. Practical orthodontics, 6th ed. St. Louis: CV Mosby Company; 1942. p. 498-514.

Dinçer M, Meral O, Tümer N. The investigation of occlusal contacts during the retention period. Angle Orthod 2003;73:640-6.

Downs WB. Variations in facial relationships (their significance in treatment and prognosis). Am J Orthod 1948;34:812-40.

Driscoll-Gilliland J, Buschang PH, Behrents RG. An evaluation of growth and stability in untreated and treated subjects. Am J Orthod Dentofacial Orthop 2001;120:588-97.

Edwards JG. A long-term prospective evaluation of the circumferential supracrestal fiberotomy in alleviating orthodontic relapse. Am J Orthod Dentofacial Orthop 1988;93:380-7. 
Edwards JG. A study of the periodontium during orthodontic rotation of teeth. Am J Orthod 1968;54:441-61.

Edwards JG. A surgical procedure to eliminate rotational relapse. Am J Orthod 1970;57:35-46.

Elms TN, Buschang PH, Alexander RG. Long-term stability of Class II, Division 1 nonextraction cervical face-bow therapy. Am J Orthod Dentofacial Orthop 1996;109:271-6.

Eslambolchi S, Woodside DG, Rossouw PE. A descriptive study of mandibular incisor alignment in untreated subjects. Am J Orthod Dentofacial Orthop 2008;133:343-53.

Fastlicht J. Crowding of mandibular incisors. Am J Orthod 1970;58:156-63.

Fränkel R, Fränkel C. Orofacial orthopedics with the function regulator. Basel: Karger; 1989.

Gardner RA, Harris EF, Vaden JL. Postorthodontic dental changes: a longitudinal study. Am J Orthod Dentofacial Orthop 1998;114:581-6.

Gebeck TR, Merrifield LL. Orthodontic diagnosis and treatment analysis concepts and values. Part I. Am J Orthod 1995;107:434-43.

Germane N, Lindauer SJ, Rubenstein LK, Revere JH, Isaacson RJ. Increase in arch perimeter due to orthodontic expansion. Am J Orthod Dentofacial Orthop 1991;100:421-7.

Gianelly A. Evidence-based therapy: an orthodontic dilemma. Am J Orthod Dentofacial Orthop 2006;129:596-8.

Gilmore C, Little RM. Mandibular incisor dimensions and crowding. Am J Orthod 1984;86:493-502.

Glass GV. Primary, secondary and meta-analysis of research. Educ Res 1976;5:38.

Glassell BE, Harris EF. Sex differences in the uptake of orthodontic services among adolescents. J Dent Res 2008; Abstracts 0331. 
Glenn G, Sinclair PM, Alexander RG. Nonextraction orthodontic therapy: posttreatment dental and skeletal stability. Am J Orthod Dentofacial Orthop 1987;92:321-8.

Gorman JC, Smith RJ. Comparison of treatment effects with labial and lingual fixed appliances. Am J Orthod 1991;99:202-9.

Gould M, Picton D. A method of measuring forces acting on the teeth from the lips, cheek, and tongue. Br Dent J 1962;112:235-42.

Graber TM. Postmortems in posttreatment adjustment. Am J Orthod 1966;52:331-52.

Graber TM, Vanarsdall RL. Orthodontics: current principles and techniques, 2nd ed. St. Louis: CV Mosby; 1994.

Graber TM, Vanarsdall RL, Vig KWL. Orthodontics: current principles and techniques, 4th ed. St. Louis: CV Mosby; 2005.

Guilford SH. Orthodontia, 3rd ed. Philadelphia: TC Davis and Sons; 1898.

Hahn GW. Retention-The stepchild of orthodontia. Angle Orthod 1944;14:3-12.

Harris EF. A longitudinal study of arch size and form in untreated adults. Am J Orthod Dentofacial Orthop 1997;111:419-27.

Harris EF, Behrents RG. The intrinsic stability of Class I molar relationship: a longitudinal study of untreated patients. Am J Orthod Dentofacial Orthop 1988;94:63-7.

Harris EF, Corruccini RS. Quantification of dental occlusal variation: a review of methods. Dental Anthropology 2008;21:1-11.

Harris EF, Gardner RZ, Vaden JL. A longitudinal cephalometric study of postorthodontic craniofacial changes. Am J Orthod Dentofacial Orthop 1999;115:77-82.

Harris EF, Vaden JL, Dunn KL, Behrents RG. Effects of patient age on postorthodontic stability in Class II, division 1 malocclusions. Am J Orthod Dentofacial Orthop 1994;105:25-34.

Harris GS. Graphic display of total space analysis data. J CH Tweed Found 1985;13:28-52. 
Hawley CA. A removable retainer. Int J Orthod 1919;2:291-8.

Heier EE, De Smit AA, Wijgaerts IA, Adriaens PA. Periodontal implications of bonded versus removable retainers. Am J Orthod Dentofacial Orthop 1997;112:607-16.

Hellman M. Changes in the human face brought on by development. Int J Orthod 1927;13:475-516.

Hellman M. Failures in orthodontic treatment. Int J Orthod 1936;22:343-58.

Hellman M. Fundamental principles and expedient compromises in orthodontic procedures. Am J Orthod Oral Surg 1944;30:429-36.

Herberger RJ. Stability of mandibular intercuspid width after long periods of retention. Angle Orthod 1981;51:103-15.

Holdaway RH. Changes in relationship of points A and B during orthodontic treatment. Am J Orthod 1956;42:176-93.

Horowitz SL, Hixon EH. Physiologic recovery following orthodontic treatment. Am J Orthod 1969;55:1-4.

Houston WJB, Edler R. Long-term stability of the lower labial segment relative to the A-Pog line. Eur J Orthod 1990;12:302-10.

Howes, AE. Case analysis and treatment planning based upon the relationship of tooth material to its supporting bone. Am J Orthod Oral Surg 1947;33:499533.

Huckaba GW. The physiologic basis of relapse. Am J Orthod 1952;38:335-49.

Jackson VH. Orthodontia and orthopaedia of the face. Philadelphia: Lippincott; 1904. p. 415.

Jacobson A. Application of the "Wits" appraisal. Am J Orthod 1976;70:179-89.

Jacobson A. The "Wits" appraisal of jaw disharmony. Am J Orthod 1975;67:12538.

Johnston LE, White L. JCO interviews Dr. Lysle E. Johnston, Jr., on orthodontics and the scientific method. J Clin Orthod 1993;27:201-6. 
Joondeph DR. Retention and relapse. In: Graber TM, Vanarsdall RL, Vig KWL. Orthodontics: current principles and techniques, 4th ed. St. Louis: Elsevier; 2005. p. 1123-51.

Joondeph DR, Riedel RA. Retention and relapse. In: Graber TM, Vanarsdall RL, Vig KWL. Orthodontics: current principles and techniques, 2nd ed. St. Louis: Elsevier; 1994. p. 908-50.

Kahl-Nieke B, Fischbach H, Schwarze CW. Post-retention crowding and incisor irregularity: a long-term follow-up evaluation of stability and relapse. Br J Orthod 1995;22:249-57.

Kaplan H. The logic of modern retention procedures. Am J Orthod Dentofacial Orthop 1988;93:325-40.

Katsaros C, Livas C, Renkema AM. Unexpected complications of bonded mandibular lingual retainers. Am J Orthod Dentofacial Orthop 2007;132:838-41.

Kelly JE, Harvey CR. An assessment of the occlusion of the teeth of youths 12-17 years. Vital Health Stat 1977;162:1-65.

Kessling HD. The philosophy of the tooth positioning appliance. Am J Orthod Oral Surg 1945;31:297-304.

King GJ, Keeling SD. Orthodontic bone remodeling in relation to appliance decay. Angle Orthod 1995;65:129-40.

Kingsley N. A letter to the alumni society. Angle School of Orthodontia, 1908.

Kingsley N. Treatise on oral deformities. New York: Appleton; 1880.

Knott VB. Longitudinal study of dental arch widths at four stages of dentition. Angle Orthod 1972;42:387-94.

Knott VB. Size and form of the dental arches in children with good occlusion studied longitudinally from age 9 years to late adolescence. Am J Phys Anthropol 1961;19:263-84.

Kuijpers-Jagtman AM. Repair and revision 8. Relapse of lower incisors: retreatment? Ned Tijdschr Tandheelkd 2002;109:42-6. 
Lee RT. Arch width and form: a review. Am J Orthod Dentofacial Orthop 1999;115:305-13.

Lee RT. The lower incisor bonded retainer in clinical practice: a three-year study.Br J Orthod 1981;8:15-8.

Levin MA. Post-orthodontic maintenance in the adult patient. Dent Clin North Am 1972;16:559-71.

Lindauer SJ. Orthodontic treatment planning. In: Nanda R. Biomechanics in clinical orthodontics. Philadelphia: WB Saunders Company; 1997. p. 23-49.

Lischer BE. Principles and methods of orthodontics. Philadelphia: Lea and Febiger; 1912.

Little RM. The irregularity index: a quantitative score of mandibular anterior alignment. Am J Orthod 1975;68:554-63.

Little RM. Stability and relapse: early treatment of arch length deficiency. Am J Orthod Dentofacial Orthop 2002;121:578-81.

Little RM, Riedel RA, Årtun J. An evaluation of changes in mandibular anterior alignment from 10 to 20 years postretention. Am J Orthod Dentofacial Orthop 1988;93:423-8.

Little RM, Riedel RA. Postretention evaluation of stability and relapse mandibular arches with generalized spacing. Am J Orthod DentofacialOrthop 1989;95:37-41.

Little RM, Riedel RA, Stein A. Mandibular arch length increase during the mixed dentition: postretention evaluation of stability and relapse. Am J Orthod Dentofacial Orthop 1990;97:393-404.

Little RM, Sinclair PM. Clinical implications of the University of Washington post-retention studies. J Clin Orthod 2009;43:645-51.

Little RM, Sinclair PM. Clinical implications of the University of Washington post-retention studies. J Clin Orthod 2009;44:723-7.

Little RM, Wallen TR, Riedel RA. Stability and relapse of mandibular anterior alignment-first premolar extraction cases treated by traditional edgewise orthodontics. Am J Orthod 1981;80:349-65. 
Littlewood SJ, Millett DT, Doubleday B, Bearn DR, Worthington HV.

Orthodontic retention: a systematic review. J Orthod 2006;33:205-12.

Lundström A. Changes in crowding and spacing of the teeth with age. Dent Pract 1969;19:218-24.

Lundström A. Malocclusion of the teeth regarded as a problem in correction of the apical base. Int J Orthod 1925;11:591-602.

Luppanapornlarp S, Johnston LE. The effects of premolar-extraction: a long-term comparison of outcomes in "clear-cut" extraction and nonextraction Class II patients. Angle Orthod 1993;63:257-72.

Markus MB. A review and consideration of the problem of retention. Am J Orthod Oral Surg 1938;24:203-12.

Massler M. Prevalence of malocclusion in children aged 14 to 18 years. Am J Orthod 1951;37:751-68.

McCauley DR. The cuspid and its function in retention. Am J Orthod 1944;30:196-205.

McKinney JR, Harris EF. Influence of patient age and sex on orthodontic treatment: evaluations of Begg lightwire, standard edgewise, and straightwire techniques. Am J Orthod 2001;120:530-41.

Melrose C, Millett DT. Toward a perspective on orthodontic retention? Am J Orthod Dentofacial Orthop 1998;113:507-14.

Merrifield LL. The profile line as an aid in critically evaluating facial esthetics. Am J Orthod 1966;52:804-22.

Merrifield LL. Differential diagnosis with total space analysis. Bull CH Tweed Found 1978;6:10-5.

Merrifield LL. The sequential directional force edgewise technique. In: Johnston LE. New vistas in orthodontics. Philadelphia: Lea and Febinger; 1985. p. 184-208.

Mershon JV. Proceedings of the First International Orthodontic Congress. St. Louis: CV Mosby Company; 1927. 
Mills RJE. The long term results of proclination of lower incisors. Br Dent J 1966;120:355-63.

Moore AW. The mechanism of adjustment to wear and accident in the dentition and periodontium. Angle Orthod 1956;26:50-7.

Moorrees CFA. The dentition of the growing child. Cambridge: Harvard University Press; 1959.

Moorrees CFA, Gron AM, Lebret LM, Yen PK, Frohlich FJ. Growth studies of the dentition. Am J Orthod 1969;55:600-16.

Morton S, Pancherz H. Changes in functional occlusion during the postorthodontic retention period: a prospective longitudinal clinical study. Am J Orthod Dentofacial Orthop 2009;135:310-5.

Moss ML. Functional cranial analysis of the mandibular angular cartilage in the rat. Angle Orthod 1969;39:209-14.

Moss JP. The soft tissue environment of teeth and jaws: experimental malocclusion. Br J Orthod 1980;7:205-16.

Moyers RE. Handbook of orthodontics for the student and general practitioner, 3rd ed. Chicago: YearBook; 1973. p. 442.

Moyers RE. Temporomandibular muscle contraction patterns in Angle Class II, Division 1 malocclusion: an electromyographic analysis. Am J Orthod 1949;35:837-57.

Muchnic HV. Retention or continuing treatment. Am J Orthod 1970;57:23-34.

Nanda RS. Growth changes in skeletal-facial profile and their significance in orthodontic diagnosis. Am J Orthod 1971;59:501-13.

Nanda RS, Meng H, Kapila S, Goorhuis J. Growth changes in the soft tissue profile. Angle Orthod 1990;60:216-23.

Nanda RS, Nanda SK. Considerations of dentofacial growth in long-term retention and stability: is active retention needed? Am J Orthod 1992;101:297-302.

Oppenheim A. A possibility for physiologic orthodontic movement. Am J Orthod Oral Surg 1944;30:277-328. 
Ormiston JP, Huang GJ, Little RM, Decker JD, Seuk GD. Retrospective analysis of long-term stable and unstable orthodontic treatment outcomes. Am J Orthod Dentofacial Orthop 2005;128:568-74.

Pandis N, Vlahopoulos K, Madianos P, Eliades T. Long-term periodontal status of patients with mandibular lingual fixed retention. Eur J Orthod 2007;29:471-6.

Paquette DE, Beattie JR, Johnston LE. A long-term comparison of nonextraction and premolar extraction edgewise therapy in "borderline" Class II patients. Am J Orthod Dentofacial Orthop 1992;102:1-14.

Paulson RC. A functional rationale for routine maxillary bonded retention. Angle Orthod 1992;62:223-6.

Peck S, Peck H. Crown dimensions and mandibular incisor alignment. Angle Orthod 1972;42:148-53.

Perera PSG. Rotational growth and incisor compensation. Angle Orthod 1987;57:39-49.

Proffit WR. Equilibrium theory revisited: factors influencing position of the teeth. Angle Orthod 1978;48:175-86.

Proffit WR. Retention. In: Contemporary orthodontics, 2nd ed. St. Louis: CV Mosby; 1993. p. 534-5.

Proffit WR, Kydd WL, Wilshire GH, Taylor DT. Intraoral pressures in a young adult group. J Dent Res 1964;43:555-62.

Puneky PJ, Sadowsky C, BeGole EA. Tooth morphology and lower incisor alignment many years after orthodontic therapy. Am J Orthod 1984;86:299-305.

Razdolsky Y, Sadowsky C, BeGole EA. Occlusal contacts following orthodontic treatment: a follow-up study. Angle Orthod 1989;59:181-5.

Redlich M, Rahamim E, Gaft A, Shochan S. The response of supraalveolar gingival collagen to orthodontic rotation movement in dogs. Am J Orthod Dentofacial Orthop 1996;110:247-55.

Reitan K. Clinical and histological observations on tooth movement during and after orthodontic treatment. Am J Orthod 1967;53:721-45. 
Reitan K. Experiments of rotation of teeth and their subsequent retention. Trans Eur Orthod Soc 1958;34:124-40.

Reitan K. Principles of retention and avoidance of posttreatment relapse. Am J Orthod 1969;55:776-90.

Reitan K. Tissue rearrangement during retention of orthodontically rotated teeth. Angle Orthod 1959;29:105-13.

Renkema AM, Al-Assad S, Bronkhorst E, Weindel S, Katsaros C, Lisson JA. Effectiveness of lingual retainers bonded to the canines in preventing mandibular incisor relapse. Am J Orthod Dentofacial Orthop 2008;134:179.e1-179.e8.

Richardson ME. Late lower arch crowding in relation to primary crowding. Angle Orthod 1982;52:300-12.

Richardson ME. Late third molar genesis: its significance in orthodontic treatment. Angle Orthod 1980;50:121-8.

Richardson ME. A review of changes in lower arch alignment from seven to fifty years. Semin Orthod 1999;5:151-9.

Richardson ME. The role of the third molar in the cause of late lower arch crowding - a review. Am J Orthod Dentofacial Orthop 1989;95:79-83.

Ricketts RM. A principle of arcial growth of the mandible. Angle Orthod 1972;42:368-86.

Ricketts RM, Bench RW, Hilgers JJ, Schulhof R. An overview of computerized cephalometrics. Am J Orthod 1972;61:1-28.

Ricketts RM, Roth RH, Chaconas SJ, Schulhof RJ, Engel GA. Orthodontic diagnosis and planning. USA: Rocky Mountain Data Systems; 1982. p. 194-200.

Riedel RA. A review of the retention problem. Angle Orthod 1960;30:179-94.

Riedel RA, Brandt S. Dr. Richard Riedel on retention and relapse. J Clin Orthod 1976;10:454-72.

Rinchuse DJ, Miles P, Sheridan JJ. Orthodontic retention and stability: a clinical perspective. J Clin Orthod 2007;41:125-32. 
Rosenstein SW, Jacobson BN. Retention: an equal partner. Am J Orthod 1971;59:323-32.

Rowland H, Hichens L, Williams A, Hills D, Killingback N, Ewings P, Clark S, Ireland AJ, Sandy JR. The effectiveness of Hawley and vacuum-formed retainers: a single-center randomized controlled trial. Am J Orthod Dentofacial Orthop 2007;132:730-7.

Rygh P, Bowling K, Hovlandsdal L, Williams, S. Activation of the vascular system: a main mediator of periodontal fiber remodeling in orthodontic tooth movement. Am J Orthod Dentofacial Orthop 1986;89:453-68.

Sadowsky C, Sakols EI. Long-term assessment of orthodontic relapse. Am J Orthod 1982;82:456-63.

Sadowsky C, Schneider BJ, BeGole EA, Tahir E. Long-term stability after orthodontic treatment: nonextraction with prolonged retention. Am J Orthod Dentofacial Orthop 1994;106:243-9.

Sandusky WC, Gramling JF. The edgewise technique. In: Hardin JF. Clark's clinical dentistry, Vol. 2. Philadelphia: Lippencott Company; 1988. Chapter 27.

Sanin C, Savara BS. Factors that affect the alignment of the mandibular incisors: a longitudinal study. Am J Orthod 1973;64:248-57.

Sauget E, Covell DA, Boero RP, Lieber WS. Comparison of occlusal contacts with use of Hawley and clear overlay retainers. Angle Orthod 1997;67:223-30.

Shanley LS. The influence of mandibular third molars on mandibular anterior teeth. Am J Orthod 1962;48:787-8.

Sheneman J. Third molar teeth and their effect upon the lower anterior teeth: a study of forty-nine orthodontic cases five years after band removal. Am J Orthod 1969;55:196 (Abstract).

Shah AA. Postretention changes in mandibular crowding: a review of the literature. Am J Orthod 2003;124:298-308.

Shaw WC. Factors influencing the desire for orthodontic treatment. Eur J Orthod 1981;3:151-62. 
Shaw WC, O'Brien KD, Richmond S. Quality control in orthodontics: factors influencing the receipt of orthodontic treatment. British Dental J 1991;170:66-8.

Shields TE, Little RM, Chapko MK. Stability and relapse of mandibular anterior alignment: a cephalometric appraisal of first premolar extraction cases treated by traditional edgewise orthodontics. Am J Orthod 1985;87:27-38.

Siatkowski RE. Incisor uprighting: mechanism for late secondary crowding in the anterior segments of the dental arches. Am J Orthod 1974;66:398-410.

Sillman JH. Dimensional changes of the dental arches: longitudinal study from birth to 25 years. Am J Orthod 1964;50:824-42.

Sinclair PM, Little RM. Dentofacial maturation of untreated normals. Am J Orthod 1985;88:146-56.

Sinclair PM, Little RM. Maturation of untreated normal occlusions. Am J Orthod 1983;83:114-23.

Sloss EA, Southard KA, Qian F, Stock SE, Mann KR, Meyer DL, Southard TE. Comparison of soft-tissue profiles after treatment with headgear or Herbst appliance. Am J Orthod Dentofacial Orthop 2008;133:509-14.

Smith RJ, Davidson WM, Gipe GP. Incisor shape and incisor crowding: a reevaluation of the Peck and Peck ratio. Am J Orthod 1982;82:231-5.

Sokal RR, Rohlf FJ. Biometry: the principles and practice of statistics in biological research, 3rd ed. San Francisco: WH Freeman and Company; 1995.

Southard TE, Southard KA, Tolley EA. Periodontal force: a potential cause of relapse. Am J Orthod Dentofacial Orthop 1992;101:221-7.

Strang R. Factors associated with successful orthodontic treatment. Am J Orthod 1952;38:790-800.

Strang R. The fallacy of denture expansion as a treatment procedure. Angle Orthod 1949;19:12-22.

Steadman SR. Changes of intermolar and intercuspid distances following orthodontic treatment. Angle Orthod 1961;31:207-15. 
Steadman SR. A philosophy and practice of orthodontic retention. Angle Orthod 1967;37:175-85.

Stephens CK, Boley JC, Behrents RG, Alexander RG, Buschang PH. Long-term profile changes in extraction and nonextraction patients. Am J Orthod 2005;128:450-7.

Stoner MM, Lindquist JT. A cephalometric evaluation of fifty-seven consecutive cases treated by Dr. Charles H. Tweed. Angle Orthod 1956;26:68-98.

Sullivan B, Freer TJ, Vautin D, Basford KE. Occlusal contacts: comparison of orthodontic patients, post-treatment patients, and untreated controls. J Prosthet Dent 1991;65:232-7.

Tanne K, Inone Y, Sakuda M. Biomechanical behavior of the periodontium before and after orthodontic tooth movement. Angle Orthod 1995;65:123-8.

Tanner JM. Growth at adolescence, 2nd ed. Oxford: Blackwell Scientific Publications; 1962.

Thilander B. Orthodontic relapse versus natural development. Am J Orthod Dentofacial Orthop 2000;117:562-3.

Thompson HE. Orthodontic relapses analyzed in a study of connective tissue fibers. Am J Orthod 1959;45:93-103.

Tirk T. Limitations in orthodontic treatment. Angle Orthod 1965;35:165-77.

Tukey JW. Exploratory data analysis. Reading, MA: Addision-Wesley; 1977.

Tweed $\mathrm{CH}$. The application of the principles of the edge-wise arch in the treatment of malocclusions: Angle Orthod 1941;11:5-11.

Tweed $\mathrm{CH}$. Indications for the extraction of teeth in orthodontic procedures. Am J Orthod Oral Surg 1944;30:405-28.

Tweed CH. A philosophy of orthodontic treatment. Am J Orthod 1945;31:74-103.

Tweed $\mathrm{CH}$. Why I extract teeth in the treatment of certain types of malocclusion. Alpha Omegan 1952;16:93-104.

Tweed CH. Clinical orthodontics, Vol. I and II. St. Louis: CV Mosby; 1966. 
Ursi WJ, Trotman CA, McNamara JA Jr, Behrents RG. Sexual dimorphism in normal craniofacial growth. Angle Orthod 1993;63:47-56.

Vaden JL, Dale JG, Klontz HA. The Tweed-Merrifield edgewise appliance: philosophy, diagnosis, and treatment. In: Graber TM, Vanarsdall RL Jr. Orthodontics: current principles and techniques. St. Louis: Mosby; 2000. p. 647-707.

Vaden JL, Harris EF, Gardner RL. Relapse revisited. Am J Orthod Dentofacial Orthop 1997;111:543-53.

van der Linden F. Facial growth and facial orthopedics. Chicago: Quintessence Publishing; 1986.

Waldron R. Reviewing the problem of retention. Am J Orthod Oral Surg 1942;28:770-91.

Walter DC. Changes in form and dimensions of dental arches resulting from orthodontic treatment. Angle Orthod 1953;23:3-18.

Wasson JL. Achieving functional occlusion with orthodontics. In: Clark JW. Clark's clinical dentistry, Vol. 2. Philadelphia: Lippincott Company; 1988. p. 31-9.

Webster RL. Retention. Am J Orthod 1948;34:897-937.

Weinberger BW. Orthodontics: a historical review of its origin and evolution, Vol. I and II. St. Louis: CV Mosby Company; 1926.

Weinstein S, Haack DC, Morris LY, Synder BB, Attaway HE. On an equilibrium theory of tooth position. Angle Orthod 1963;33:1-26.

Winer BJ, Brown DR, Michels KM. Statistical principles in experimental design, 3rd ed. New York: McGraw-Hill Book Company; 1991.

Zachrisson BU. Clinical experience with direct-bonded orthodontic retainers. Am J Orthod 1977;71:440-8.

Zachrisson BU. Important aspects of long-term stability. J Clin Orthod 1997;31:562-83.

Zachrisson BU. Improving orthodontic results in cases with maxillary incisors missing. Am J Orthod 1978;73:274-89. 
Zachrisson BU, Büyükyilmaz T. Bonding in orthodontics. In: Graber TM, Vanarsdall RL, Vig KWL. Orthodontics: current principles and techniques, 4th ed. St. Louis: Elsevier Inc.; 2005. p. 579-659. 
APPENDIX A. DESCRIPTIVE STATISTICS FOR ALL VARIABLES 
Table A-1. Descriptive statistics at the start of treatment (sexes pooled).

\begin{tabular}{|c|c|c|c|c|c|c|c|c|}
\hline \multirow[b]{2}{*}{ Variable } & \multicolumn{4}{|c|}{ Fixed Retention } & \multicolumn{4}{|c|}{ Removable Retention } \\
\hline & $\bar{x}$ & sd & sem & $\mathrm{n}$ & $\bar{x}$ & sd & sem & $\mathrm{n}$ \\
\hline ADisc & 1.66 & 3.25 & 0.391 & 69 & 1.92 & 2.52 & 0.256 & 97 \\
\hline MADisc & 0.87 & 3.29 & 0.396 & 69 & 0.43 & 3.25 & 0.330 & 97 \\
\hline cos & 2.20 & 0.75 & 0.090 & 69 & 2.18 & 0.94 & 0.095 & 97 \\
\hline $\mathrm{MC}$ & 6.68 & 5.19 & 0.625 & 69 & 6.26 & 4.66 & 0.473 & 97 \\
\hline ASR & 33.89 & 5.47 & 0.658 & 69 & 32.99 & 5.30 & 0.538 & 97 \\
\hline ISR & 16.99 & 7.88 & 0.949 & 69 & 15.56 & 6.35 & 0.644 & 97 \\
\hline IAR & 76.97 & 15.46 & 1.861 & 69 & 72.81 & 17.24 & 1.750 & 97 \\
\hline ASP & 35.39 & 3.11 & 0.374 & 69 & 34.45 & 2.83 & 0.287 & 97 \\
\hline UMW & 51.19 & 3.25 & 0.391 & 69 & 50.38 & 3.34 & 0.339 & 97 \\
\hline LMW & 50.54 & 2.95 & 0.356 & 69 & 50.27 & 2.68 & 0.272 & 97 \\
\hline UCW & 32.58 & 2.57 & 0.349 & 54 & 32.44 & 2.26 & 0.255 & 78 \\
\hline LCW & 24.64 & 2.16 & 0.268 & 65 & 24.44 & 2.02 & 0.215 & 88 \\
\hline ISD & 3.54 & 1.63 & 0.196 & 69 & 3.24 & 1.32 & 0.134 & 97 \\
\hline ASD & 9.22 & 1.56 & 0.188 & 69 & 8.83 & 1.42 & 0.145 & 97 \\
\hline $\mathrm{ID}^{*}$ & 25.52 & 7.71 & 0.928 & 69 & 21.89 & 7.29 & 0.740 & 97 \\
\hline ASW & 27.16 & 1.86 & 0.224 & 69 & 26.74 & 1.86 & 0.189 & 97 \\
\hline II & 5.36 & 3.86 & 0.464 & 69 & 5.91 & 3.80 & 0.386 & 97 \\
\hline FMIA & 60.29 & 6.04 & 0.727 & 69 & 60.18 & 6.81 & 0.691 & 97 \\
\hline FMA & 26.70 & 5.87 & 0.707 & 69 & 27.02 & 5.69 & 0.577 & 97 \\
\hline
\end{tabular}


Table A-1. Continued.

\begin{tabular}{|c|c|c|c|c|c|c|c|c|}
\hline \multirow[b]{2}{*}{ Variable } & \multicolumn{4}{|c|}{ Fixed Retention } & \multicolumn{4}{|c|}{ Removable Retention } \\
\hline & $\bar{x}$ & sd & sem & $\mathrm{n}$ & $\bar{x}$ & sd & sem & $\mathrm{n}$ \\
\hline IMPA & 93.13 & 7.21 & 0.868 & 69 & 92.92 & 7.10 & 0.721 & 97 \\
\hline SNA* & 81.12 & 3.06 & 0.368 & 69 & 80.16 & 3.30 & 0.335 & 97 \\
\hline SNB & 76.36 & 2.94 & 0.353 & 69 & 75.90 & 3.55 & 0.360 & 97 \\
\hline ANB & 4.75 & 2.33 & 0.280 & 69 & 4.27 & 2.30 & 0.234 & 97 \\
\hline $\mathrm{AOBO}$ & 2.94 & 4.06 & 0.489 & 69 & 1.88 & 3.71 & 0.377 & 97 \\
\hline DOP & 8.46 & 4.02 & 0.484 & 69 & 9.49 & 3.86 & 0.392 & 97 \\
\hline Z angle & 68.86 & 8.90 & 1.072 & 69 & 68.92 & 8.93 & 0.907 & 97 \\
\hline UL & 13.28 & 2.66 & 0.320 & 69 & 13.19 & 2.40 & 0.243 & 97 \\
\hline $\mathrm{TC}^{*}$ & 14.74 & 2.67 & 0.321 & 69 & 13.67 & 2.34 & 0.238 & 97 \\
\hline $\mathrm{AFH}$ & 64.13 & 5.37 & 0.646 & 69 & 63.58 & 5.65 & 0.574 & 97 \\
\hline PFH & 47.35 & 4.50 & 0.542 & 69 & 47.30 & 5.39 & 0.547 & 97 \\
\hline $\mathrm{OB}$ & 4.35 & 2.53 & 0.304 & 69 & 3.86 & 2.07 & 0.210 & 97 \\
\hline OJ* & 6.28 & 3.34 & 0.402 & 69 & 5.03 & 2.63 & 0.267 & 97 \\
\hline
\end{tabular}

${ }^{*}$ An asterisk denotes statistical significance between the two kinds of retention. 
Table A-2. Descriptive statistics at the end of treatment (sexes pooled).

\begin{tabular}{|c|c|c|c|c|c|c|c|c|}
\hline \multirow[b]{2}{*}{ Variable } & \multicolumn{4}{|c|}{ Fixed Retention } & \multicolumn{4}{|c|}{ Removable Retention } \\
\hline & $\overline{\mathrm{x}}$ & $\mathrm{sd}$ & sem & $\mathrm{n}$ & $\overline{\mathrm{x}}$ & $\mathrm{sd}$ & sem & $\mathrm{n}$ \\
\hline ADisc & -0.08 & 0.69 & 0.083 & 69 & -0.33 & 0.57 & 0.058 & 97 \\
\hline MADisc & -0.27 & 0.32 & 0.038 & 69 & -0.39 & 0.51 & 0.052 & 97 \\
\hline $\operatorname{COS}^{*}$ & 0.75 & 0.66 & 0.079 & 69 & 0.44 & 0.55 & 0.056 & 97 \\
\hline$M C^{*}$ & 1.49 & 1.68 & 0.202 & 69 & 0.99 & 1.24 & 0.126 & 97 \\
\hline ASR & 34.80 & 2.96 & 0.356 & 69 & 34.00 & 3.76 & 0.381 & 97 \\
\hline ISR & 17.07 & 2.70 & 0.325 & 69 & 17.81 & 2.89 & 0.293 & 97 \\
\hline IAR & 76.67 & 12.94 & 1.558 & 69 & 75.34 & 12.66 & 1.285 & 97 \\
\hline ASP & 37.65 & 2.07 & 0.249 & 69 & 36.96 & 2.61 & 0.265 & 97 \\
\hline UMW & 50.68 & 2.86 & 0.345 & 69 & 50.72 & 2.99 & 0.303 & 97 \\
\hline LMW & 48.36 & 2.69 & 0.323 & 69 & 48.43 & 3.12 & 0.316 & 97 \\
\hline UCW & 33.72 & 1.82 & 0.219 & 69 & 33.11 & 2.00 & 0.203 & 97 \\
\hline $\mathrm{LCW}$ & 25.91 & 1.39 & 0.167 & 69 & 25.53 & 1.72 & 0.175 & 97 \\
\hline ISD & 3.75 & 0.59 & 0.072 & 69 & 3.83 & 0.70 & 0.071 & 97 \\
\hline ASD & 9.99 & 0.93 & 0.112 & 69 & 9.66 & 1.16 & 0.118 & 97 \\
\hline $\mathrm{ID}^{*}$ & 24.02 & 7.62 & 0.918 & 69 & 19.99 & 6.88 & 0.699 & 97 \\
\hline ASW & 28.68 & 1.33 & 0.161 & 69 & 28.40 & 1.76 & 0.179 & 97 \\
\hline $\mathrm{II}^{*}$ & 0.46 & 0.70 & 0.084 & 69 & 0.24 & 0.35 & 0.036 & 97 \\
\hline FMIA & 62.38 & 5.87 & 0.707 & 69 & 62.63 & 6.43 & 0.653 & 97 \\
\hline FMA & 26.14 & 6.62 & 0.797 & 69 & 26.89 & 5.82 & 0.591 & 97 \\
\hline
\end{tabular}


Table A-2. Continued.

\begin{tabular}{|c|c|c|c|c|c|c|c|c|}
\hline \multirow[b]{2}{*}{ Variable } & \multicolumn{4}{|c|}{ Fixed Retention } & \multicolumn{4}{|c|}{ Removable Retention } \\
\hline & $\overline{\mathrm{x}}$ & sd & sem & $\mathrm{n}$ & $\overline{\mathrm{x}}$ & sd & sem & $\mathrm{n}$ \\
\hline IMPA & 91.59 & 7.75 & 0.933 & 69 & 90.60 & 6.80 & 0.690 & 97 \\
\hline SNA & 79.06 & 3.36 & 0.405 & 69 & 78.47 & 3.38 & 0.343 & 97 \\
\hline SNB & 76.32 & 3.12 & 0.375 & 69 & 75.69 & 3.64 & 0.369 & 97 \\
\hline ANB & 2.74 & 1.69 & 0.203 & 69 & 2.78 & 1.93 & 0.196 & 97 \\
\hline $\mathrm{AOBO}$ & 0.64 & 2.35 & 0.282 & 69 & 0.37 & 2.39 & 0.243 & 97 \\
\hline DOP & 9.49 & 3.88 & 0.467 & 69 & 10.05 & 3.88 & 0.394 & 97 \\
\hline Z angle & 76.12 & 6.51 & 0.784 & 69 & 75.14 & 6.51 & 0.661 & 97 \\
\hline UL & 15.48 & 2.97 & 0.357 & 69 & 14.95 & 2.84 & 0.288 & 97 \\
\hline $\mathrm{TC}^{*}$ & 16.41 & 2.38 & 0.286 & 69 & 15.36 & 2.31 & 0.234 & 97 \\
\hline $\mathrm{AFH}$ & 68.43 & 5.35 & 0.644 & 69 & 67.09 & 5.56 & 0.564 & 97 \\
\hline PFH & 52.32 & 5.14 & 0.619 & 69 & 50.71 & 4.67 & 0.474 & 97 \\
\hline $\mathrm{OB}^{*}$ & 2.51 & 1.16 & 0.139 & 69 & 2.00 & 0.84 & 0.085 & 97 \\
\hline $\mathrm{OJ}^{*}$ & 2.67 & 0.74 & 0.089 & 69 & 2.32 & 0.80 & 0.081 & 97 \\
\hline
\end{tabular}

${ }^{*}$ An asterisk denotes statistical significance between the two kinds of retention. 
Table A-3. Descriptive statistics for the recall examination (sexes pooled).

\begin{tabular}{|c|c|c|c|c|c|c|c|c|}
\hline \multirow[b]{2}{*}{ Variable } & \multicolumn{4}{|c|}{ Fixed Retention } & \multicolumn{4}{|c|}{ Removable Retention } \\
\hline & $\bar{x}$ & $\mathrm{sd}$ & sem & $\mathrm{n}$ & $\bar{x}$ & $\mathrm{sd}$ & sem & $\mathrm{n}$ \\
\hline ADisc & 0.38 & 1.43 & 0.172 & 69 & 0.56 & 1.48 & 0.150 & 97 \\
\hline MADisc & -0.15 & 0.28 & 0.034 & 69 & -0.19 & 0.58 & 0.059 & 97 \\
\hline $\mathrm{COS}^{*}$ & 1.19 & 0.56 & 0.068 & 69 & 1.01 & 0.58 & 0.059 & 97 \\
\hline $\mathrm{MC}$ & 1.59 & 1.73 & 0.208 & 69 & 1.30 & 1.86 & 0.189 & 97 \\
\hline ASR & 34.42 & 4.20 & 0.506 & 69 & 33.26 & 4.92 & 0.499 & 97 \\
\hline ISR & 17.77 & 4.56 & 0.548 & 69 & 17.02 & 5.00 & 0.507 & 97 \\
\hline IAR & 82.44 & 15.87 & 1.911 & 69 & 78.46 & 14.20 & 1.442 & 97 \\
\hline ASP & 36.08 & 2.31 & 0.278 & 69 & 35.27 & 2.60 & 0.264 & 97 \\
\hline UMW & 50.29 & 3.20 & 0.385 & 69 & 49.90 & 3.06 & 0.311 & 97 \\
\hline LMW & 47.99 & 2.98 & 0.359 & 69 & 47.98 & 3.33 & 0.338 & 97 \\
\hline UCW & 33.27 & 2.04 & 0.245 & 69 & 32.61 & 2.10 & 0.213 & 97 \\
\hline LCW & 24.60 & 1.54 & 0.186 & 69 & 24.44 & 1.77 & 0.180 & 97 \\
\hline ISD & 3.69 & 0.93 & 0.112 & 69 & 3.50 & 1.02 & 0.104 & 97 \\
\hline ASD & 9.50 & 1.16 & 0.140 & 69 & 9.07 & 1.31 & 0.133 & 97 \\
\hline $\mathrm{ID}^{*}$ & 23.87 & 7.76 & 0.934 & 69 & 19.96 & 6.77 & 0.687 & 97 \\
\hline ASW & 27.58 & 1.49 & 0.179 & 69 & 27.32 & 1.77 & 0.180 & 97 \\
\hline $\mathrm{II}^{*}$ & 1.92 & 2.03 & 0.244 & 69 & 2.53 & 2.55 & 0.259 & 97 \\
\hline FMIA & 63.55 & 6.11 & 0.736 & 69 & 64.42 & 6.93 & 0.703 & 97 \\
\hline FMA & 25.20 & 7.15 & 0.861 & 69 & 26.29 & 6.42 & 0.652 & 97 \\
\hline
\end{tabular}


Table A-3. Continued.

\begin{tabular}{|c|c|c|c|c|c|c|c|c|}
\hline \multirow[b]{2}{*}{ Variable } & \multicolumn{4}{|c|}{ Fixed Retention } & \multicolumn{4}{|c|}{ Removable Retention } \\
\hline & $\bar{x}$ & $\mathrm{sd}$ & sem & $\mathrm{n}$ & $\bar{x}$ & $\mathrm{sd}$ & sem & $\mathrm{n}$ \\
\hline IMPA* & 91.42 & 7.33 & 0.882 & 69 & 89.45 & 6.73 & 0.684 & 97 \\
\hline SNA & 79.19 & 3.34 & 0.403 & 69 & 78.48 & 3.26 & 0.331 & 97 \\
\hline SNB & 76.32 & 3.26 & 0.392 & 69 & 75.56 & 3.61 & 0.366 & 97 \\
\hline ANB & 2.87 & 1.87 & 0.225 & 69 & 2.93 & 2.06 & 0.209 & 97 \\
\hline AOBO & 1.67 & 2.48 & 0.298 & 69 & 1.67 & 2.81 & 0.285 & 97 \\
\hline DOP & 7.93 & 4.31 & 0.519 & 69 & 8.57 & 4.42 & 0.449 & 97 \\
\hline $\mathrm{Z}$ angle & 79.62 & 7.67 & 0.924 & 69 & 78.63 & 8.22 & 0.835 & 97 \\
\hline UL & 13.55 & 2.88 & 0.346 & 69 & 12.97 & 2.62 & 0.266 & 97 \\
\hline TC & 17.52 & 2.74 & 0.329 & 69 & 16.56 & 2.92 & 0.297 & 97 \\
\hline $\mathrm{AFH}$ & 69.75 & 5.62 & 0.677 & 69 & 68.44 & 6.10 & 0.619 & 97 \\
\hline PFH & 54.12 & 5.86 & 0.706 & 69 & 52.47 & 5.56 & 0.564 & 97 \\
\hline OB & 3.29 & 1.33 & 0.160 & 69 & 3.14 & 1.27 & 0.129 & 97 \\
\hline OJ & 3.20 & 1.02 & 0.123 & 69 & 2.95 & 1.15 & 0.117 & 97 \\
\hline
\end{tabular}

${ }^{*}$ An asterisk denotes statistical significance between the two kinds of retention. 
Table A-4. Descriptive statistics for the in-treatment changes (sexes pooled).

\begin{tabular}{|c|c|c|c|c|c|c|c|c|}
\hline \multirow[b]{2}{*}{ Variable } & \multicolumn{4}{|c|}{ Fixed Retention } & \multicolumn{4}{|c|}{ Removable Retention } \\
\hline & $\bar{x}$ & $\mathrm{sd}$ & sem & $\mathrm{n}$ & $\bar{x}$ & $\mathrm{sd}$ & sem & $\mathrm{n}$ \\
\hline ADisc & -1.74 & 2.97 & 0.358 & 69 & -2.25 & 2.60 & 0.264 & 97 \\
\hline MADisc & -1.14 & 3.29 & 0.397 & 69 & -0.82 & 3.37 & 0.342 & 97 \\
\hline $\mathrm{COS}^{*}$ & -1.45 & 0.95 & 0.114 & 69 & -1.74 & 1.04 & 0.106 & 97 \\
\hline $\mathrm{MC}$ & -5.19 & 5.28 & 0.636 & 69 & -5.27 & 4.85 & 0.492 & 97 \\
\hline ASR & 0.91 & 5.49 & 0.661 & 69 & 1.01 & 4.87 & 0.495 & 97 \\
\hline ISR $^{*}$ & 0.09 & 7.94 & 0.956 & 69 & 2.25 & 6.03 & 0.612 & 97 \\
\hline IAR & -0.31 & 14.13 & 1.702 & 69 & 2.52 & 18.96 & 1.925 & 97 \\
\hline ASP & 2.27 & 2.84 & 0.342 & 69 & 2.50 & 2.80 & 0.284 & 97 \\
\hline UMW & -0.51 & 2.28 & 0.274 & 69 & 0.35 & 2.42 & 0.246 & 97 \\
\hline LMW & -2.18 & 1.88 & 0.227 & 69 & -1.84 & 2.27 & 0.230 & 97 \\
\hline UCW & 1.06 & 2.11 & 0.287 & 54 & 0.65 & 1.94 & 0.220 & 78 \\
\hline LCW & 1.30 & 2.31 & 0.286 & 65 & 0.98 & 1.52 & 0.162 & 88 \\
\hline ISD & 0.21 & 1.63 & 0.196 & 69 & 0.59 & 1.27 & 0.129 & 97 \\
\hline ASD & 0.78 & 1.50 & 0.181 & 69 & 0.83 & 1.40 & 0.142 & 97 \\
\hline ID & -1.50 & 2.24 & 0.270 & 69 & -1.89 & 2.70 & 0.274 & 97 \\
\hline ASW & 1.52 & 1.72 & 0.207 & 69 & 1.65 & 1.70 & 0.173 & 97 \\
\hline II & -4.89 & 3.70 & 0.446 & 69 & -5.67 & 3.80 & 0.386 & 97 \\
\hline FMIA & 2.09 & 6.30 & 0.759 & 69 & 2.45 & 5.89 & 0.599 & 97 \\
\hline FMA & -0.55 & 2.28 & 0.274 & 69 & -0.13 & 2.05 & 0.208 & 97 \\
\hline
\end{tabular}


Table A-4. Continued.

\begin{tabular}{|c|c|c|c|c|c|c|c|c|}
\hline \multirow[b]{2}{*}{ Variable } & \multicolumn{4}{|c|}{ Fixed Retention } & \multicolumn{4}{|c|}{ Removable Retention } \\
\hline & $\bar{x}$ & $\mathrm{sd}$ & sem & $\mathrm{n}$ & $\bar{x}$ & $\mathrm{sd}$ & sem & $\mathrm{n}$ \\
\hline IMPA & -1.54 & 5.52 & 0.664 & 69 & -2.32 & 6.11 & 0.620 & 97 \\
\hline SNA & -2.06 & 1.86 & 0.224 & 69 & -1.69 & 1.61 & 0.163 & 97 \\
\hline SNB & -0.04 & 1.53 & 0.184 & 69 & -0.21 & 1.41 & 0.143 & 97 \\
\hline ANB & -2.01 & 1.92 & 0.231 & 69 & -1.48 & 1.86 & 0.189 & 97 \\
\hline AOBO & -2.30 & 3.32 & 0.400 & 69 & -1.51 & 2.94 & 0.299 & 97 \\
\hline DOP & 1.03 & 2.67 & 0.322 & 69 & 0.56 & 2.74 & 0.278 & 97 \\
\hline $\mathrm{Z}$ angle & 7.26 & 6.04 & 0.727 & 69 & 6.23 & 6.48 & 0.658 & 97 \\
\hline UL & 2.20 & 2.73 & 0.328 & 69 & 1.76 & 2.42 & 0.246 & 97 \\
\hline TC & 1.67 & 1.88 & 0.227 & 69 & 1.69 & 1.79 & 0.181 & 97 \\
\hline $\mathrm{AFH}$ & 4.30 & 2.99 & 0.360 & 69 & 3.52 & 2.82 & 0.286 & 97 \\
\hline PFH & 4.97 & 3.68 & 0.443 & 69 & 3.41 & 3.56 & 0.362 & 97 \\
\hline OB & -1.84 & 2.51 & 0.302 & 69 & -1.86 & 2.19 & 0.222 & 97 \\
\hline OJ & -3.61 & 3.37 & 0.406 & 69 & -2.71 & 2.65 & 0.269 & 97 \\
\hline
\end{tabular}

${ }^{*}$ An asterisk denotes statistical significance between the two kinds of retention. 
Table A-5. Descriptive statistics for the posttreatment changes (sexes pooled).

\begin{tabular}{|c|c|c|c|c|c|c|c|c|}
\hline \multirow[b]{2}{*}{ Variable } & \multicolumn{4}{|c|}{ Fixed Retention } & \multicolumn{4}{|c|}{ Removable Retention } \\
\hline & $\bar{x}$ & $\mathrm{sd}$ & sem & $\mathrm{n}$ & $\bar{x}$ & $\mathrm{sd}$ & sem & $\mathrm{n}$ \\
\hline ADisc $^{*}$ & 0.46 & 1.26 & 0.152 & 69 & 0.89 & 1.41 & 0.143 & 97 \\
\hline MADisc & 0.12 & 0.31 & 0.038 & 69 & 0.20 & 0.74 & 0.075 & 97 \\
\hline COS & 0.44 & 0.63 & 0.076 & 69 & 0.58 & 0.67 & 0.068 & 97 \\
\hline $\mathrm{MC}$ & 0.10 & 1.71 & 0.206 & 69 & 0.31 & 1.86 & 0.189 & 97 \\
\hline ASR & -0.38 & 3.14 & 0.378 & 69 & -0.74 & 3.42 & 0.347 & 97 \\
\hline $\mathrm{ISR}^{*}$ & 0.69 & 3.96 & 0.477 & 69 & -0.80 & 4.27 & 0.434 & 97 \\
\hline $\mathrm{IAR}^{*}$ & 5.77 & 12.41 & 1.494 & 69 & 3.12 & 12.78 & 1.297 & 97 \\
\hline ASP & -1.58 & 1.18 & 0.142 & 69 & -1.69 & 1.43 & 0.145 & 97 \\
\hline UMW* & -0.39 & 0.86 & 0.104 & 69 & -0.82 & 0.98 & 0.099 & 97 \\
\hline LMW & -0.37 & 0.96 & 0.115 & 69 & -0.45 & 1.16 & 0.118 & 97 \\
\hline UCW & -0.45 & 0.99 & 0.119 & 69 & -0.51 & 1.27 & 0.129 & 97 \\
\hline $\mathrm{LCW}$ & -1.31 & 1.06 & 0.128 & 69 & -1.09 & 1.01 & 0.103 & 97 \\
\hline ISD* & -0.05 & 0.76 & 0.091 & 69 & -0.33 & 0.84 & 0.085 & 97 \\
\hline ASD & -0.50 & 0.73 & 0.088 & 69 & -0.59 & 0.82 & 0.084 & 97 \\
\hline ID & -0.16 & 1.38 & 0.167 & 69 & -0.03 & 1.92 & 0.195 & 97 \\
\hline ASW & -1.10 & 0.97 & 0.117 & 69 & -1.08 & 1.08 & 0.110 & 97 \\
\hline $\mathrm{II}^{*}$ & 1.46 & 1.90 & 0.229 & 69 & 2.30 & 2.60 & 0.264 & 97 \\
\hline FMIA & 1.17 & 3.68 & 0.443 & 69 & 1.79 & 4.05 & 0.412 & 97 \\
\hline FMA & -0.94 & 2.00 & 0.241 & 69 & -0.60 & 2.10 & 0.214 & 97 \\
\hline
\end{tabular}


Table A-5. Continued.

\begin{tabular}{|c|c|c|c|c|c|c|c|c|}
\hline \multirow[b]{2}{*}{ Variable } & \multicolumn{4}{|c|}{ Fixed Retention } & \multicolumn{4}{|c|}{ Removable Retention } \\
\hline & $\bar{x}$ & $\mathrm{sd}$ & sem & $\mathrm{n}$ & $\bar{x}$ & $\mathrm{sd}$ & sem & $\mathrm{n}$ \\
\hline IMPA & -0.17 & 3.44 & 0.414 & 69 & -1.14 & 3.76 & 0.382 & 97 \\
\hline SNA & 0.13 & 1.11 & 0.134 & 69 & 0.01 & 1.31 & 0.133 & 97 \\
\hline SNB & 0.00 & 1.03 & 0.124 & 69 & -0.13 & 1.24 & 0.126 & 97 \\
\hline ANB & 0.13 & 1.22 & 0.147 & 69 & 0.14 & 1.27 & 0.129 & 97 \\
\hline АОВО & 1.03 & 1.81 & 0.218 & 69 & 1.30 & 1.91 & 0.194 & 97 \\
\hline DOP & -1.57 & 2.13 & 0.257 & 69 & -1.48 & 2.18 & 0.222 & 97 \\
\hline $\mathrm{Z}$ angle & 3.51 & 3.89 & 0.468 & 69 & 3.48 & 4.52 & 0.459 & 97 \\
\hline UL & -1.93 & 1.89 & 0.227 & 69 & -1.98 & 2.04 & 0.207 & 97 \\
\hline TC & 1.12 & 1.84 & 0.222 & 69 & 1.20 & 1.75 & 0.178 & 97 \\
\hline $\mathrm{AFH}$ & 1.32 & 2.38 & 0.286 & 69 & 1.35 & 2.19 & 0.223 & 97 \\
\hline PFH & 1.80 & 3.07 & 0.369 & 69 & 1.76 & 3.07 & 0.312 & 97 \\
\hline $\mathrm{OB}$ & 0.78 & 1.25 & 0.150 & 69 & 1.14 & 1.31 & 0.134 & 97 \\
\hline OJ & 0.54 & 1.07 & 0.128 & 69 & 0.63 & 1.11 & 0.113 & 97 \\
\hline
\end{tabular}

${ }^{*}$ An asterisk denotes statistical significance between the two kinds of retention. 
APPENDIX B. ANCOVA TESTS OF THE COMPARABILITY OF CASES (FIXED VERSUS REMOVABLE RETENTION) WHILE CONTROLLING FOR SEXUAL DIMORPHISM 
Table B-1. Results of two-way ANOVA tests evaluating the comparability of cases (fixed versus removable retention) while controlling for sexual dimorphism at the start of orthodontic treatment. ${ }^{1}$

\begin{tabular}{|c|c|c|c|c|c|c|}
\hline \multirow[b]{2}{*}{ Variable } & \multicolumn{2}{|c|}{ Sex } & \multicolumn{2}{|c|}{ Retention } & \multicolumn{2}{|c|}{ Interaction } \\
\hline & $\mathrm{F}$ & $\mathrm{P}$ & $\mathrm{F}$ & $\mathrm{P}$ & $\mathrm{F}$ & $\mathrm{P}$ \\
\hline ADisc & 1.29 & 0.2581 & 1.45 & 0.2303 & 3.24 & 0.0736 \\
\hline MADisc & 0.15 & 0.7005 & 0.09 & 0.7631 & 1.21 & 0.2725 \\
\hline cos & 11.75 & 0.0008 & 0.26 & 0.6143 & 0.21 & 0.6480 \\
\hline $\mathrm{MC}$ & 7.09 & 0.0085 & 0.29 & 0.5932 & 0.73 & 0.3941 \\
\hline ASR & 5.80 & 0.0171 & 0.26 & 0.6128 & 0.09 & 0.7642 \\
\hline ISR & 8.15 & 0.0049 & 1.86 & 0.1749 & 1.52 & 0.2196 \\
\hline IAR & 6.46 & 0.0120 & 2.58 & 0.1099 & 1.18 & 0.2780 \\
\hline ASP & 10.74 & 0.0013 & 3.52 & 0.0625 & 1.05 & 0.3065 \\
\hline UMW & 8.48 & 0.0041 & 2.34 & 0.1282 & 1.10 & 0.2953 \\
\hline LMW & 5.57 & 0.0194 & 0.83 & 0.3637 & 2.10 & 0.1496 \\
\hline UCW & 1.76 & 0.1864 & 0.09 & 0.7609 & 0.24 & 0.6245 \\
\hline LCW & 1.12 & 0.2916 & 0.01 & 0.9378 & 0.71 & 0.4009 \\
\hline ISD & 10.62 & 0.0014 & 1.75 & 0.1881 & 1.39 & 0.2409 \\
\hline ASD & 10.16 & 0.0017 & 1.66 & 0.1994 & 0.15 & 0.6958 \\
\hline ID & 12.55 & 0.0005 & 8.17 & 0.0048 & 1.16 & 0.2832 \\
\hline ASW & 3.42 & 0.0660 & 2.64 & 0.1058 & 1.73 & 0.1906 \\
\hline II & 0.04 & 0.8324 & 0.43 & 0.5121 & 0.07 & 0.7933 \\
\hline FMIA & 0.81 & 0.3705 & 0.34 & 0.5607 & 0.65 & 0.4222 \\
\hline FMA & 0.17 & 0.6830 & 0.79 & 0.3750 & 1.85 & 0.1755 \\
\hline IMPA & 1.26 & 0.2638 & 0.03 & 0.8706 & 0.09 & 0.7621 \\
\hline SNA & 0.09 & 0.7648 & 4.50 & 0.0354 & 1.21 & 0.2733 \\
\hline SNB & 2.65 & 0.1052 & 2.03 & 0.1563 & 0.86 & 0.3545 \\
\hline ANB & 7.74 & 0.0060 & 0.87 & 0.3513 & 0.04 & 0.8355 \\
\hline AOBO & 23.87 & $<0.0001$ & 1.32 & 0.2523 & 0.08 & 0.7832 \\
\hline DOP & 4.86 & 0.0289 & 2.54 & 0.1127 & 0.73 & 0.3932 \\
\hline $\mathrm{Z}$ angle & 12.64 & 0.0005 & 0.63 & 0.4302 & 0.68 & 0.4113 \\
\hline UL & 2.70 & 0.1022 & 0.00 & 0.9863 & 0.00 & 0.9557 \\
\hline
\end{tabular}

Continued 
Table B-1. Continued.

\begin{tabular}{|c|c|c|c|c|c|c|}
\hline \multirow[b]{2}{*}{ Variable } & \multicolumn{2}{|c|}{ Sex } & \multicolumn{2}{|c|}{ Retention } & \multicolumn{2}{|c|}{ Interaction } \\
\hline & $\mathrm{F}$ & $\mathrm{P}$ & $\mathrm{F}$ & $\mathrm{P}$ & $\mathrm{F}$ & $\mathrm{P}$ \\
\hline $\mathrm{TC}$ & 0.84 & 0.3598 & 6.77 & 0.0101 & 0.48 & 0.4897 \\
\hline AFH & 0.62 & 0.4316 & 0.24 & 0.6285 & 0.00 & 0.9494 \\
\hline PFH & 0.60 & 0.4402 & 0.40 & 0.5272 & 2.17 & 0.1430 \\
\hline $\mathrm{OB}$ & 21.75 & $<0.0001$ & 0.18 & 0.6713 & 0.33 & 0.5638 \\
\hline OJ & 16.17 & $<0.0001$ & 6.06 & 0.0148 & 1.22 & 0.2713 \\
\hline
\end{tabular}

${ }^{1}$ There is $1 \mathrm{df}$ associated with each of the three tests for a variable. 
Table B-2. Results of two-way ANOVA tests evaluating the comparability of cases (fixed versus removable retention) while controlling for sexual dimorphism at the end of orthodontic treatment. ${ }^{1}$

\begin{tabular}{|c|c|c|c|c|c|c|}
\hline \multirow[b]{2}{*}{ Variable } & \multicolumn{2}{|c|}{ Sex } & \multicolumn{2}{|c|}{ Retention } & \multicolumn{2}{|c|}{ Interaction } \\
\hline & $\mathrm{F}$ & $\mathrm{P}$ & $\mathrm{F}$ & $\mathrm{P}$ & $\mathrm{F}$ & $\mathrm{P}$ \\
\hline ADisc & 0.08 & 0.7729 & 1.59 & 0.2095 & 4.69 & 0.0319 \\
\hline MADisc & 0.23 & 0.6344 & 2.15 & 0.1441 & 0.01 & 0.9035 \\
\hline cos & 0.02 & 0.8751 & 14.40 & 0.0002 & 3.35 & 0.0692 \\
\hline MC & 1.18 & 0.2793 & 6.98 & 0.0091 & 1.49 & 0.2239 \\
\hline ASR & 2.51 & 0.1152 & 0.24 & 0.6272 & 1.80 & 0.1814 \\
\hline ISR & 0.41 & 0.5239 & 2.88 & 0.0917 & 0.11 & 0.7458 \\
\hline IAR & 3.32 & 0.0705 & 0.01 & 0.9100 & 0.27 & 0.6052 \\
\hline ASP & 8.66 & 0.0037 & 0.52 & 0.4713 & 1.46 & 0.2282 \\
\hline UMW & 11.27 & 0.0010 & 0.31 & 0.5760 & 5.38 & 0.0216 \\
\hline LMW & 8.38 & 0.0043 & 0.00 & 0.9846 & 1.30 & 0.2553 \\
\hline UCW & 10.76 & 0.0013 & 1.52 & 0.2187 & 0.09 & 0.7608 \\
\hline LCW & 3.75 & 0.0546 & 0.36 & 0.5480 & 1.14 & 0.2879 \\
\hline ISD & 1.35 & 0.2478 & 1.49 & 0.2243 & 0.75 & 0.3886 \\
\hline ASD & 7.17 & 0.0082 & 0.60 & 0.4406 & 2.10 & 0.1488 \\
\hline ID & 8.13 & 0.0049 & 12.62 & 0.0005 & 2.38 & 0.1250 \\
\hline ASW & 4.87 & 0.0287 & 0.27 & 0.6025 & 0.20 & 0.6585 \\
\hline II & 0.08 & 0.7754 & 6.72 & 0.0104 & 0.18 & 0.6721 \\
\hline FMIA & 2.52 & 0.1147 & 0.11 & 0.7361 & 0.04 & 0.8430 \\
\hline FMA & 1.07 & 0.3015 & 1.05 & 0.3070 & 1.11 & 0.2944 \\
\hline IMPA & 0.20 & 0.6547 & 1.34 & 0.2495 & 0.51 & 0.4747 \\
\hline SNA & 1.71 & 0.1925 & 1.88 & 0.1722 & 0.27 & 0.6065 \\
\hline SNB & 0.27 & 0.6036 & 1.58 & 0.2099 & 0.14 & 0.7049 \\
\hline ANB & 2.05 & 0.1545 & 0.02 & 0.8768 & 0.06 & 0.8136 \\
\hline AOBO & 3.28 & 0.0721 & 0.14 & 0.7110 & 0.00 & 0.9500 \\
\hline DOP & 3.90 & 0.0501 & 0.57 & 0.4532 & 0.18 & 0.6752 \\
\hline $\mathrm{Z}$ angle & 2.17 & 0.1430 & 1.08 & 0.3011 & 0.00 & 0.9718 \\
\hline UL & 40.11 & $<0.0001$ & 0.40 & 0.5274 & 0.43 & 0.5117 \\
\hline
\end{tabular}

Continued 
Table B-2. Continued.

\begin{tabular}{|c|c|c|c|c|c|c|}
\hline \multirow[b]{2}{*}{ Variable } & \multicolumn{2}{|c|}{ Sex } & \multicolumn{2}{|c|}{ Retention } & \multicolumn{2}{|c|}{ Interaction } \\
\hline & $\mathrm{F}$ & $\mathrm{P}$ & $\mathrm{F}$ & $\mathrm{P}$ & $\mathrm{F}$ & $\mathrm{P}$ \\
\hline TC & 12.43 & 0.0005 & 5.81 & 0.0171 & 0.37 & 0.5456 \\
\hline AFH & 11.54 & 0.0009 & 0.69 & 0.4068 & 0.10 & 0.7525 \\
\hline PFH & 19.76 & $<0.0001$ & 2.82 & 0.0949 & 0.43 & 0.5138 \\
\hline OB & 1.04 & 0.3094 & 10.14 & 0.0017 & 0.16 & 0.6860 \\
\hline OJ & 0.00 & 0.9527 & 5.67 & 0.0184 & 0.05 & 0.8299 \\
\hline
\end{tabular}

${ }^{1}$ There is $1 \mathrm{df}$ associated with each of the three tests for a variable. 
Table B-3. Results of two-way ANOVA tests evaluating the comparability of cases (fixed versus removable retention) while controlling for sexual dimorphism at the recall examination. ${ }^{1}$

\begin{tabular}{|c|c|c|c|c|c|c|}
\hline \multirow[b]{2}{*}{ Variable } & \multicolumn{2}{|c|}{ Sex } & \multicolumn{2}{|c|}{ Retention } & \multicolumn{2}{|c|}{ Interaction } \\
\hline & $\mathrm{F}$ & $\mathrm{P}$ & $\mathrm{F}$ & $\mathrm{P}$ & $\mathrm{F}$ & $\mathrm{P}$ \\
\hline ADisc & 0.24 & 0.6259 & 3.56 & 0.0609 & 7.03 & 0.0088 \\
\hline MADisc & 0.06 & 0.8144 & 0.08 & 0.7755 & 0.13 & 0.7182 \\
\hline cos & 0.09 & 0.7654 & 4.30 & 0.0396 & 0.43 & 0.5126 \\
\hline MC & 0.00 & 0.9497 & 0.45 & 0.5048 & 0.22 & 0.6385 \\
\hline ASR & 0.02 & 0.8789 & 1.34 & 0.2479 & 0.21 & 0.6498 \\
\hline ISR & 0.00 & 0.9733 & 1.69 & 0.1958 & 0.86 & 0.3550 \\
\hline IAR & 4.46 & 0.0362 & 3.15 & 0.0780 & 1.36 & 0.2460 \\
\hline ASP & 8.76 & 0.0035 & 2.06 & 0.1532 & 0.01 & 0.9333 \\
\hline UMW & 16.47 & $<.0001$ & 2.22 & 0.1383 & 7.45 & 0.0071 \\
\hline LMW & 13.60 & 0.0003 & 0.08 & 0.7833 & 2.58 & 0.1105 \\
\hline UCW & 26.01 & $<.0001$ & 1.62 & 0.2050 & 0.00 & 0.9742 \\
\hline LCW & 11.54 & 0.0009 & 0.03 & 0.8577 & 0.37 & 0.5417 \\
\hline ISD & 0.34 & 0.5626 & 1.75 & 0.1875 & 0.39 & 0.5348 \\
\hline ASD & 2.86 & 0.0929 & 2.44 & 0.1203 & 0.08 & 0.7713 \\
\hline ID & 7.50 & 0.0069 & 12.65 & 0.0005 & 3.03 & 0.0837 \\
\hline ASW & 10.10 & 0.0018 & 0.46 & 0.5000 & 0.15 & 0.6979 \\
\hline II & 0.67 & 0.4130 & 4.95 & 0.0275 & 2.09 & 0.1498 \\
\hline FMIA & 10.96 & 0.0011 & 1.39 & 0.2395 & 0.00 & 0.9968 \\
\hline FMA & 5.52 & 0.0200 & 1.30 & 0.2555 & 1.31 & 0.2546 \\
\hline IMPA & 0.44 & 0.5072 & 4.78 & 0.0302 & 1.33 & 0.2514 \\
\hline SNA & 2.66 & 0.1049 & 2.33 & 0.1292 & 0.07 & 0.7926 \\
\hline SNB & 0.73 & 0.3936 & 1.53 & 0.2183 & 0.07 & 0.7937 \\
\hline ANB & 19.57 & $<0.0001$ & 0.14 & 0.7055 & 0.00 & 0.9808 \\
\hline AOBO & 0.04 & 0.8485 & 0.05 & 0.8203 & 0.28 & 0.5959 \\
\hline DOP & 11.24 & 0.0010 & 0.21 & 0.6467 & 0.01 & 0.9204 \\
\hline $\mathrm{Z}$ angle & 0.89 & 0.3460 & 0.54 & 0.4626 & 0.13 & 0.7219 \\
\hline UL & 57.49 & $<0.0001$ & 0.18 & 0.6687 & 0.00 & 0.9726 \\
\hline
\end{tabular}

Continued 
Table B-3. Continued.

\begin{tabular}{|c|c|c|c|c|c|c|}
\hline \multirow[b]{2}{*}{ Variable } & \multicolumn{2}{|c|}{ Sex } & \multicolumn{2}{|c|}{ Retention } & \multicolumn{2}{|c|}{ Interaction } \\
\hline & $\mathrm{F}$ & $\mathrm{P}$ & $\mathrm{F}$ & $\mathrm{P}$ & $\mathrm{F}$ & $\mathrm{P}$ \\
\hline $\mathrm{TC}$ & 38.04 & $<0.0001$ & 1.44 & 0.2314 & 0.05 & 0.8179 \\
\hline $\mathrm{AFH}$ & 22.93 & $<0.0001$ & 0.04 & 0.8469 & 1.27 & 0.2613 \\
\hline PFH & 71.14 & $<0.0001$ & 0.50 & 0.4801 & 0.10 & 0.7566 \\
\hline $\mathrm{OB}$ & 1.72 & 0.1911 & 1.11 & 0.2934 & 0.32 & 0.5753 \\
\hline OJ & 0.05 & 0.8180 & 2.27 & 0.1339 & 0.29 & 0.5904 \\
\hline
\end{tabular}

${ }^{1}$ There is $1 \mathrm{df}$ associated with each of the three tests for a variable. 
Table B-4. Results of two-way ANOVA tests evaluating the comparability of cases (fixed versus removable retention) while controlling for sexual dimorphism for the in-treatment changes. ${ }^{1}$

\begin{tabular}{|c|c|c|c|c|c|c|}
\hline \multirow[b]{2}{*}{ Variable } & \multicolumn{2}{|c|}{ Sex } & \multicolumn{2}{|c|}{ Retention } & \multicolumn{2}{|c|}{ Interaction } \\
\hline & $\mathrm{F}$ & $\mathrm{P}$ & $\mathrm{F}$ & P & $\mathrm{F}$ & $\mathrm{P}$ \\
\hline ADisc & 1.21 & 0.2733 & 2.31 & 0.1309 & 1.85 & 0.1753 \\
\hline MADisc & 0.19 & 0.6602 & 0.01 & 0.9201 & 1.13 & 0.2900 \\
\hline cos & 9.20 & 0.0028 & 7.53 & 0.0068 & 2.27 & 0.1340 \\
\hline MC & 8.47 & 0.0041 & 0.06 & 0.8048 & 0.23 & 0.6341 \\
\hline ASR & 2.06 & 0.1534 & 0.04 & 0.842 & 0.34 & 0.5594 \\
\hline ISR & 6.96 & 0.0092 & 4.40 & 0.0376 & 1.93 & 0.1664 \\
\hline IAR & 1.13 & 0.2892 & 2.09 & 0.1501 & 2.02 & 0.1569 \\
\hline ASP & 0.79 & 0.3755 & 1.73 & 0.1896 & 4.28 & 0.0402 \\
\hline UMW & 0.00 & 0.9850 & 2.03 & 0.1565 & 1.76 & 0.1860 \\
\hline LMW & 0.75 & 0.3883 & 1.33 & 0.2504 & 0.11 & 0.7434 \\
\hline UCW & 0.25 & 0.6189 & 0.44 & 0.5106 & 0.37 & 0.5428 \\
\hline LCW & 0.23 & 0.6333 & 1.29 & 0.2576 & 0.14 & 0.7080 \\
\hline ISD & 7.55 & 0.0067 & 3.65 & 0.0578 & 2.55 & 0.1123 \\
\hline ASD & 1.57 & 0.2113 & 0.54 & 0.4641 & 2.12 & 0.1473 \\
\hline ID & 4.96 & 0.0274 & 3.01 & 0.0847 & 1.51 & 0.2204 \\
\hline ASW & 0.00 & 0.9634 & 1.63 & 0.2029 & 3.38 & 0.0680 \\
\hline II & 0.03 & 0.8609 & 1.06 & 0.3053 & 0.04 & 0.8358 \\
\hline FMIA & 6.89 & 0.0095 & 0.97 & 0.3256 & 0.45 & 0.5027 \\
\hline FMA & 3.57 & 0.0608 & 0.31 & 0.5782 & 0.41 & 0.5252 \\
\hline IMPA & 3.73 & 0.0551 & 1.52 & 0.2192 & 0.27 & 0.6060 \\
\hline SNA & 10.33 & 0.0016 & 1.72 & 0.1915 & 1.15 & 0.2858 \\
\hline SNB & 6.21 & 0.0137 & 0.06 & 0.8068 & 1.49 & 0.2239 \\
\hline ANB & 25.76 & $<0.0001$ & 1.09 & 0.2976 & 0.00 & 0.9816 \\
\hline AOBO & 20.83 & $<0.0001$ & 1.25 & 0.2657 & 0.15 & 0.6971 \\
\hline DOP & 0.12 & 0.7249 & 1.47 & 0.2269 & 0.39 & 0.5308 \\
\hline $\mathrm{Z}$ angle & 11.96 & 0.0007 & 0.00 & 0.9908 & 1.27 & 0.2610 \\
\hline UL & 27.29 & $<0.0001$ & 0.46 & 0.499 & 0.44 & 0.5078 \\
\hline
\end{tabular}

Continued 
Table B-4. Continued.

\begin{tabular}{|c|c|c|c|c|c|c|}
\hline \multirow[b]{2}{*}{ Variable } & \multicolumn{2}{|c|}{ Sex } & \multicolumn{2}{|c|}{ Retention } & \multicolumn{2}{|c|}{ Interaction } \\
\hline & $\mathrm{F}$ & $\mathrm{P}$ & $\mathrm{F}$ & $\mathrm{P}$ & $\mathrm{F}$ & P \\
\hline TC & 10.19 & 0.0017 & 0.33 & 0.5681 & 0.04 & 0.8439 \\
\hline AFH & 25.77 & $<0.0001$ & 0.41 & 0.5222 & 0.57 & 0.4520 \\
\hline PFH & 24.38 & $<0.0001$ & 1.84 & 0.1767 & 1.69 & 0.1957 \\
\hline OB & 25.56 & $<0.0001$ & 1.06 & 0.3052 & 0.57 & 0.4521 \\
\hline OJ & 15.73 & 0.0001 & 3.19 & 0.0759 & 1.33 & 0.2511 \\
\hline
\end{tabular}

${ }^{1}$ There is $1 \mathrm{df}$ associated with each of the three tests for a variable. 
Table B-5. Results of two-way ANOVA tests evaluating the comparability of cases (fixed versus removable retention) while controlling for sexual dimorphism for the posttreatment changes. ${ }^{1}$

\begin{tabular}{|c|c|c|c|c|c|c|}
\hline \multirow[b]{2}{*}{ Variable } & \multicolumn{2}{|c|}{ Sex } & \multicolumn{2}{|c|}{ Retention } & \multicolumn{2}{|c|}{ Interaction } \\
\hline & F & $\mathrm{P}$ & $\mathrm{F}$ & P & $\mathrm{F}$ & $\mathrm{P}$ \\
\hline ADisc & 0.15 & 0.6981 & 6.74 & 0.0103 & 3.37 & 0.0683 \\
\hline MADisc & 0.03 & 0.8699 & 0.73 & 0.3940 & 0.04 & 0.8430 \\
\hline COS & 0.01 & 0.9046 & 2.61 & 0.1083 & 1.17 & 0.2814 \\
\hline $\mathrm{MC}$ & 0.87 & 0.3519 & 2.07 & 0.1523 & 2.12 & 0.1476 \\
\hline ASR & 2.04 & 0.1555 & 1.28 & 0.2589 & 0.56 & 0.4544 \\
\hline ISR & 0.22 & 0.6360 & 7.13 & 0.0083 & 1.70 & 0.1944 \\
\hline IAR & 0.41 & 0.5247 & 3.88 & 0.0506 & 3.58 & 0.0601 \\
\hline ASP & 0.05 & 0.8173 & 1.85 & 0.1754 & 3.96 & 0.0483 \\
\hline UMW & 7.22 & 0.0080 & 9.37 & 0.0026 & 2.61 & 0.1080 \\
\hline LMW & 7.92 & 0.0055 & 0.56 & 0.4561 & 2.39 & 0.1243 \\
\hline UCW & 10.82 & 0.0012 & 0.02 & 0.8984 & 0.32 & 0.5742 \\
\hline LCW & 5.93 & 0.0159 & 1.48 & 0.2249 & 0.45 & 0.5028 \\
\hline ISD & 0.05 & 0.8157 & 6.84 & 0.0098 & 2.15 & 0.1446 \\
\hline ASD & 0.79 & 0.3762 & 2.12 & 0.1471 & 2.18 & 0.1419 \\
\hline ID & 0.22 & 0.6405 & 0.00 & 0.9890 & 0.65 & 0.4214 \\
\hline ASW & 2.58 & 0.1101 & 0.07 & 0.7940 & 1.67 & 0.1987 \\
\hline II & 0.80 & 0.3735 & 8.02 & 0.0052 & 2.42 & 0.1218 \\
\hline FMIA & 8.98 & 0.0032 & 2.07 & 0.1523 & 0.11 & 0.7431 \\
\hline FMA & 22.50 & $<0.0001$ & 0.42 & 0.5174 & 0.33 & 0.5694 \\
\hline IMPA & 0.15 & 0.7008 & 3.60 & 0.0594 & 0.62 & 0.4336 \\
\hline SNA & 0.59 & 0.4444 & 0.10 & 0.7534 & 0.50 & 0.4800 \\
\hline SNB & 18.76 & $<0.0001$ & 0.00 & 0.9708 & 0.13 & 0.7190 \\
\hline ANB & 23.52 & $<0.0001$ & 0.13 & 0.7163 & 0.16 & 0.6858 \\
\hline АOBO & 6.76 & 0.0102 & 0.65 & 0.4204 & 0.48 & 0.4881 \\
\hline DOP & 9.95 & 0.0019 & 0.20 & 0.6549 & 0.32 & 0.5728 \\
\hline$Z$ angle & 17.72 & $<0.0001$ & 0.04 & 0.8362 & 0.42 & 0.5199 \\
\hline UL & 0.49 & 0.4849 & 0.10 & 0.7480 & 0.68 & 0.4120 \\
\hline
\end{tabular}

Continued 
Table B-5. Continued.

\begin{tabular}{|c|c|c|c|c|c|c|}
\hline \multirow[b]{2}{*}{ Variable } & \multicolumn{2}{|c|}{ Sex } & \multicolumn{2}{|c|}{ Retention } & \multicolumn{2}{|c|}{ Interaction } \\
\hline & $\mathrm{F}$ & $\mathrm{P}$ & $\mathrm{F}$ & $\mathrm{P}$ & $\mathrm{F}$ & P \\
\hline $\mathrm{TC}$ & 22.10 & $<0.0001$ & 1.96 & 0.1633 & 1.36 & 0.2449 \\
\hline AFH & 15.35 & 0.0001 & 2.42 & 0.1220 & 4.48 & 0.0358 \\
\hline PFH & 55.35 & $<0.0001$ & 2.75 & 0.0993 & 2.90 & 0.0906 \\
\hline OB & 0.28 & 0.5946 & 1.90 & 0.1701 & 0.06 & 0.8016 \\
\hline OJ & 0.04 & 0.8498 & 0.03 & 0.8610 & 0.48 & 0.4879 \\
\hline
\end{tabular}

${ }^{1}$ There is $1 \mathrm{df}$ associated with each of the three tests for a variable 


\section{APPENDIX C. ANCOVA TESTS EVALUATING WHETHER THE AMOUNT OF CHANGE DEPENDS ON THE TYPE OF RETENTION, WITH PATIENT'S SEX AND TIME-OUT-OF-TREATMENT AS COVARIATES}


Table C-1. Results of ANCOVA tests of whether the amount of relapse depends on the kind of retention; dependent variable is Anterior Discrepancy (ADisc), with time-out-of-treatment as a covariate.

\begin{tabular}{lcccc}
\hline \multicolumn{1}{c}{ Source } & df & Sum Squares & F Ratio & P Value \\
\hline Retention & 1 & 10.45 & 5.89 & 0.0163 \\
Sex & 1 & 0.45 & 0.25 & 0.6149 \\
Time & 1 & 2.95 & 1.66 & 0.1991 \\
Retention-x-Sex & 1 & 4.77 & 2.69 & 0.1028 \\
Retention-x-Time & 1 & 6.00 & 3.39 & 0.0677 \\
Sex-x-Time & 1 & 3.30 & 1.86 & 0.1744 \\
Retention-x-Sex-x-Time & 1 & 0.98 & 0.55 & 0.4591 \\
\hline
\end{tabular}

Table C-2. Results of ANCOVA tests of whether the amount of relapse depends on the kind of retention; dependent variable is Midarch Discrepancy (MADisc), with time-out-of-treatment as a covariate.

\begin{tabular}{lcccc}
\hline \multicolumn{1}{c}{ Source } & df & Sum Squares & F Ratio & P Value \\
\hline Retention & 1 & 0.31 & 0.88 & 0.3503 \\
Sex & 1 & 0.01 & 0.02 & 0.8860 \\
Time & 1 & 0.23 & 0.64 & 0.4234 \\
Retention-x-Sex & 1 & 0.02 & 0.06 & 0.8059 \\
Retention-x-Time & 1 & 1.06 & 3.00 & 0.0853 \\
Sex-x-Time & 1 & 0.01 & 0.03 & 0.8567 \\
Retention-x-Sex-x-Time & 1 & 0.01 & 0.02 & 0.8948 \\
\hline
\end{tabular}

Table C-3. Results of ANCOVA tests of whether the amount of relapse depends on the kind of retention; dependent variable is Curve of Spee (COS), with timeout-of-treatment as a covariate.

\begin{tabular}{lcccc}
\hline \multicolumn{1}{c}{ Source } & df & Sum Squares & F Ratio & P Value \\
\hline Retention & 1 & 1.32 & 3.04 & 0.0834 \\
Sex & 1 & 0.00 & 0.00 & 0.9958 \\
Time & 1 & 0.45 & 1.04 & 0.3106 \\
Retention-x-Sex & 1 & 0.63 & 1.46 & 0.2289 \\
Retention-x-Time & 1 & 0.05 & 0.12 & 0.7265 \\
Sex-x-Time & 1 & 0.49 & 1.14 & 0.2882 \\
Retention-x-Sex-x-Time & 1 & 0.47 & 1.08 & 0.3000 \\
\hline
\end{tabular}


Table C-4. Results of ANCOVA tests of whether the amount of relapse depends on the kind of retention; dependent variable is Molar Correction (MC), with time-out-of-treatment as a covariate.

\begin{tabular}{lcccc}
\hline \multicolumn{1}{c}{ Source } & df & Sum Squares & F Ratio & P Value \\
\hline Retention & 1 & 7.50 & 2.30 & 0.1312 \\
Sex & 1 & 1.95 & 0.60 & 0.4406 \\
Time & 1 & 1.05 & 0.32 & 0.5702 \\
Retention-x-Sex & 1 & 8.28 & 2.54 & 0.1129 \\
Retention-x-Time & 1 & 1.12 & 0.34 & 0.5587 \\
Sex-x-Time & 1 & 3.92 & 1.20 & 0.2741 \\
Retention-x-Sex-x-Time & 1 & 2.85 & 0.88 & 0.3506 \\
\hline
\end{tabular}

Table C-5. Results of ANCOVA tests of whether the amount of relapse depends on the kind of retention; dependent variable is Anterior Segment Ratio (ASR), with time-out-of-treatment as a covariate.

\begin{tabular}{lcccc}
\hline \multicolumn{1}{c}{ Source } & df & Sum Squares & F Ratio & P Value \\
\hline Retention & 1 & 16.35 & 1.50 & 0.2220 \\
Sex & 1 & 22.64 & 2.08 & 0.1511 \\
Time & 1 & 8.49 & 0.78 & 0.3782 \\
Retention-x-Sex & 1 & 4.03 & 0.37 & 0.5434 \\
Retention-x-Time & 1 & 16.69 & 1.53 & 0.2173 \\
Sex-x-Time & 1 & 14.91 & 1.37 & 0.2435 \\
Retention-x-Sex-x-Time & 1 & 0.94 & 0.09 & 0.7686 \\
\hline
\end{tabular}

Table C-6. Results of ANCOVA tests of whether the amount of relapse depends on the kind of retention; dependent variable is Incisor Segment Ratio (ISR), with time-out-of-treatment as a covariate.

\begin{tabular}{lcccc}
\hline \multicolumn{1}{c}{ Source } & df & Sum Squares & F Ratio & P Value \\
\hline Retention & 1 & 109.61 & 6.57 & 0.0113 \\
Sex & 1 & 1.18 & 0.07 & 0.7908 \\
Time & 1 & 14.53 & 0.87 & 0.3522 \\
Retention-x-Sex & 1 & 20.02 & 1.20 & 0.2750 \\
Retention-x-Time & 1 & 95.02 & 5.70 & 0.0182 \\
Sex-x-Time & 1 & 47.07 & 2.82 & 0.0950 \\
Retention-x-Sex-x-Time & 1 & 35.05 & 2.10 & 0.1492 \\
\hline
\end{tabular}


Table C-7. Results of ANCOVA tests of whether the amount of relapse depends on the kind of retention; dependent variable is Incisor Aspect Ratio (IAR), with time-out-of-treatment as a covariate.

\begin{tabular}{lcccc}
\hline \multicolumn{1}{c}{ Source } & df & Sum Squares & F Ratio & P Value \\
\hline Retention & 1 & 636.63 & 4.02 & 0.0467 \\
Sex & 1 & 67.85 & 0.43 & 0.5137 \\
Time & 1 & 52.02 & 0.33 & 0.5674 \\
Retention-x-Sex & 1 & 483.17 & 3.05 & 0.0827 \\
Retention-x-Time & 1 & 111.28 & 0.70 & 0.4032 \\
Sex-x-Time & 1 & 190.16 & 1.20 & 0.2749 \\
Retention-x-Sex-x-Time & 1 & 5.97 & 0.04 & 0.8463 \\
\hline
\end{tabular}

Table C-8. Results of ANCOVA tests of whether the amount of relapse depends on the kind of retention; dependent variable is Anterior Segment Perimeter (ASP), with time-out-of-treatment as a covariate.

\begin{tabular}{lcccc}
\hline \multicolumn{1}{c}{ Source } & df & Sum Squares & F Ratio & P Value \\
\hline Retention & 1 & 2.44 & 1.47 & 0.2274 \\
Sex & 1 & 0.27 & 0.16 & 0.6880 \\
Time & 1 & 3.05 & 1.84 & 0.1771 \\
Retention-x-Sex & 1 & 5.13 & 3.09 & 0.0807 \\
Retention-x-Time & 1 & 13.18 & 7.93 & 0.0055 \\
Sex-x-Time & 1 & 6.37 & 3.83 & 0.0520 \\
Retention-x-Sex-x-Time & 1 & 1.82 & 1.10 & 0.2966 \\
\hline
\end{tabular}

Table C-9. Results of ANCOVA tests of whether the amount of relapse depends on the kind of retention; dependent variable is Maxillary Intermolar Width (UMW), with time-out-of-treatment as a covariate.

\begin{tabular}{lcccc}
\hline \multicolumn{1}{c}{ Source } & df & Sum Squares & F Ratio & P Value \\
\hline Retention & 1 & 6.94 & 8.28 & 0.0046 \\
Sex & 1 & 6.32 & 7.54 & 0.0067 \\
Time & 1 & 0.76 & 0.91 & 0.3411 \\
Retention-x-Sex & 1 & 1.78 & 2.12 & 0.1471 \\
Retention-x-Time & 1 & 0.06 & 0.08 & 0.7825 \\
Sex-x-Time & 1 & 0.86 & 1.03 & 0.3116 \\
Retention-x-Sex-x-Time & 1 & 0.59 & 0.70 & 0.4029 \\
\hline
\end{tabular}


Table C-10. Results of ANCOVA tests of whether the amount of relapse depends on the kind of retention; dependent variable is Mandibular Intermolar Width (LMW), with time-out-of-treatment as a covariate.

\begin{tabular}{lcccc}
\hline \multicolumn{1}{c}{ Source } & df & Sum Squares & F Ratio & P Value \\
\hline Retention & 1 & 0.41 & 0.37 & 0.5463 \\
Sex & 1 & 8.66 & 7.73 & 0.0061 \\
Time & 1 & 1.33 & 1.19 & 0.2770 \\
Retention-x-Sex & 1 & 2.42 & 2.16 & 0.1438 \\
Retention-x-Time & 1 & 0.31 & 0.28 & 0.5989 \\
Sex-x-Time & 1 & 0.07 & 0.06 & 0.8094 \\
Retention-x-Sex-x-Time & 1 & 0.01 & 0.01 & 0.9342 \\
\hline
\end{tabular}

Table C-11. Results of ANCOVA tests of whether the amount of relapse depends on the kind of retention; dependent variable is Maxillary Intercanine Width (UCW), with time-out-of-treatment as a covariate.

\begin{tabular}{lcccc}
\hline \multicolumn{1}{c}{ Source } & df & Sum Squares & F Ratio & P Value \\
\hline Retention & 1 & 0.00 & 0.00 & 0.9874 \\
Sex & 1 & 13.18 & 10.37 & 0.0016 \\
Time & 1 & 1.52 & 1.20 & 0.2758 \\
Retention-x-Sex & 1 & 0.22 & 0.17 & 0.6810 \\
Retention-x-Time & 1 & 0.97 & 0.77 & 0.3827 \\
Sex-x-Time & 1 & 1.06 & 0.83 & 0.3633 \\
Retention-x-Sex-x-Time & 1 & 0.19 & 0.15 & 0.7022 \\
\hline
\end{tabular}

Table C-12. Results of ANCOVA tests of whether the amount of relapse depends on the kind of retention; dependent variable is Mandibular Intercanine Width (LCW), with time-out-of-treatment as a covariate.

\begin{tabular}{lcccc}
\hline \multicolumn{1}{c}{ Source } & df & Sum Squares & F Ratio & P Value \\
\hline Retention & 1 & 1.45 & 1.37 & 0.2434 \\
Sex & 1 & 6.27 & 5.93 & 0.0160 \\
Time & 1 & 0.00 & 0.00 & 0.9733 \\
Retention-x-Sex & 1 & 0.57 & 0.54 & 0.4635 \\
Retention-x-Time & 1 & 0.32 & 0.31 & 0.5804 \\
Sex-x-Time & 1 & 0.36 & 0.34 & 0.5581 \\
Retention-x-Sex-x-Time & 1 & 0.03 & 0.03 & 0.8648 \\
\hline
\end{tabular}


Table C-13. Results of ANCOVA tests of whether the amount of relapse depends on the kind of retention; dependent variable is Incisor Segment Depth (ISD), with time-out-of-treatment as a covariate.

\begin{tabular}{lcccc}
\hline \multicolumn{1}{c}{ Source } & df & Sum Squares & F Ratio & P Value \\
\hline Retention & 1 & 3.93 & 6.23 & 0.0136 \\
Sex & 1 & 0.00 & 0.00 & 0.9941 \\
Time & 1 & 0.72 & 1.15 & 0.2860 \\
Retention-x-Sex & 1 & 1.01 & 1.60 & 0.2076 \\
Retention-x-Time & 1 & 3.77 & 5.97 & 0.0156 \\
Sex-x-Time & 1 & 1.74 & 2.76 & 0.0985 \\
Retention-x-Sex-x-Time & 1 & 1.80 & 2.85 & 0.0933 \\
\hline
\end{tabular}

Table C-14. Results of ANCOVA tests of whether the amount of relapse depends on the kind of retention; dependent variable is Anterior Segment Depth (ASD), with time-out-of-treatment as a covariate.

\begin{tabular}{lcccc}
\hline \multicolumn{1}{c}{ Source } & df & Sum Squares & F Ratio & P Value \\
\hline Retention & 1 & 1.27 & 2.10 & 0.1496 \\
Sex & 1 & 0.40 & 0.67 & 0.4157 \\
Time & 1 & 0.00 & 0.00 & 0.9848 \\
Retention-x-Sex & 1 & 0.98 & 1.62 & 0.2049 \\
Retention-x-Time & 1 & 2.49 & 4.10 & 0.0447 \\
Sex-x-Time & 1 & 1.54 & 2.54 & 0.1132 \\
Retention-x-Sex-x-Time & 1 & 0.09 & 0.14 & 0.7072 \\
\hline
\end{tabular}

Table C-15. Results of ANCOVA tests of whether the amount of relapse depends on the kind of retention; dependent variable is Incisor Depth (ID), with time-outof-treatment as a covariate.

\begin{tabular}{lcccc}
\hline \multicolumn{1}{c}{ Source } & df & Sum Squares & F Ratio & P Value \\
\hline Retention & 1 & 0.00 & 0.00 & 0.9946 \\
Sex & 1 & 0.58 & 0.19 & 0.6615 \\
Time & 1 & 0.00 & 0.00 & 0.9705 \\
Retention-x-Sex & 1 & 1.23 & 0.41 & 0.5222 \\
Retention-x-Time & 1 & 3.43 & 1.14 & 0.2868 \\
Sex-x-Time & 1 & 4.54 & 1.51 & 0.2206 \\
Retention-x-Sex-x-Time & 1 & 0.06 & 0.02 & 0.8879 \\
\hline
\end{tabular}


Table C-16. Results of ANCOVA tests of whether the amount of relapse depends on the kind of retention; dependent variable is Anterior Segment Width (ASW), with time-out-of-treatment as a covariate.

\begin{tabular}{lcccc}
\hline \multicolumn{1}{c}{ Source } & df & Sum Squares & F Ratio & P Value \\
\hline Retention & 1 & 0.00 & 0.00 & 0.9832 \\
Sex & 1 & 3.43 & 3.38 & 0.0677 \\
Time & 1 & 5.47 & 5.39 & 0.0215 \\
Retention-x-Sex & 1 & 1.37 & 1.35 & 0.2476 \\
Retention-x-Time & 1 & 3.23 & 3.19 & 0.0762 \\
Sex-x-Time & 1 & 0.84 & 0.83 & 0.3636 \\
Retention-x-Sex-x-Time & 1 & 2.09 & 2.06 & 0.1528 \\
\hline
\end{tabular}

Table C-17. Results of ANCOVA tests of whether the amount of relapse depends on the kind of retention; dependent variable is Mandibular Incisor Irregularity (II), with time-out-of-treatment as a covariate.

\begin{tabular}{lcccc}
\hline \multicolumn{1}{c}{ Source } & df & Sum Squares & F Ratio & P Value \\
\hline Retention & 1 & 38.71 & 7.20 & 0.0081 \\
Sex & 1 & 3.01 & 0.56 & 0.4550 \\
Time & 1 & 7.27 & 1.35 & 0.2464 \\
Retention-x-Sex & 1 & 12.47 & 2.32 & 0.1296 \\
Retention-x-Time & 1 & 8.41 & 1.57 & 0.2127 \\
Sex-x-Time & 1 & 0.01 & 0.00 & 0.9625 \\
Retention-x-Sex-x-Time & 1 & 3.99 & 0.74 & 0.3904 \\
\hline
\end{tabular}

Table C-18. Results of ANCOVA tests of whether the amount of relapse depends on the kind of retention; dependent variable is FMIA, with time-out-of-treatment as a covariate.

\begin{tabular}{lcccc}
\hline \multicolumn{1}{c}{ Source } & df & Sum Squares & F Ratio & P Value \\
\hline Retention & 1 & 22.34 & 1.53 & 0.2175 \\
Sex & 1 & 120.16 & 8.25 & 0.0046 \\
Time & 1 & 23.89 & 1.64 & 0.2023 \\
Retention-x-Sex & 1 & 0.16 & 0.01 & 0.9177 \\
Retention-x-Time & 1 & 7.25 & 0.50 & 0.4815 \\
Sex-x-Time & 1 & 39.13 & 2.69 & 0.1033 \\
Retention-x-Sex-x-Time & 1 & 10.48 & 0.72 & 0.3977 \\
\hline
\end{tabular}


Table C-19. Results of ANCOVA tests of whether the amount of relapse depends on the kind of retention; dependent variable is FMA, with time-out-of-treatment as a covariate.

\begin{tabular}{lcccc}
\hline \multicolumn{1}{c}{ Source } & df & Sum Squares & F Ratio & P Value \\
\hline Retention & 1 & 2.99 & 0.79 & 0.3748 \\
Sex & 1 & 85.33 & 22.64 & $<0.0001$ \\
Time & 1 & 10.06 & 2.67 & 0.1044 \\
Retention-x-Sex & 1 & 2.59 & 0.69 & 0.4086 \\
Retention-x-Time & 1 & 0.22 & 0.06 & 0.8110 \\
Sex-x-Time & 1 & 12.16 & 3.23 & 0.0744 \\
Retention-x-Sex-x-Time & 1 & 0.21 & 0.06 & 0.8126 \\
\hline
\end{tabular}

Table C-20. Results of ANCOVA tests of whether the amount of relapse depends on the kind of retention; dependent variable is IMPA, with time-out-of-treatment as a covariate.

\begin{tabular}{lcccc}
\hline \multicolumn{1}{c}{ Source } & df & Sum Squares & F Ratio & P Value \\
\hline Retention & 1 & 44.47 & 3.32 & 0.0704 \\
Sex & 1 & 0.87 & 0.06 & 0.7996 \\
Time & 1 & 1.63 & 0.12 & 0.7279 \\
Retention-x-Sex & 1 & 5.96 & 0.44 & 0.5060 \\
Retention-x-Time & 1 & 15.77 & 1.18 & 0.2798 \\
Sex-x-Time & 1 & 10.44 & 0.78 & 0.3788 \\
Retention-x-Sex-x-Time & 1 & 11.05 & 0.82 & 0.3654 \\
\hline
\end{tabular}

Table C-21. Results of ANCOVA tests of whether the amount of relapse depends on the kind of retention; dependent variable is SNA, with time-out-of-treatment as a covariate.

\begin{tabular}{lcccc}
\hline \multicolumn{1}{c}{ Source } & df & Sum Squares & F Ratio & P Value \\
\hline Retention & 1 & 0.00 & 0.00 & 0.9584 \\
Sex & 1 & 0.67 & 0.44 & 0.5074 \\
Time & 1 & 5.06 & 3.33 & 0.0700 \\
Retention-x-Sex & 1 & 1.23 & 0.81 & 0.3693 \\
Retention-x-Time & 1 & 0.00 & 0.00 & 0.9790 \\
Sex-x-Time & 1 & 2.84 & 1.87 & 0.1733 \\
Retention-x-Sex-x-Time & 1 & 1.23 & 0.81 & 0.3695 \\
\hline
\end{tabular}


Table C-22. Results of ANCOVA tests of whether the amount of relapse depends on the kind of retention; dependent variable is SNB, with time-out-of-treatment as a covariate.

\begin{tabular}{lcccc}
\hline \multicolumn{1}{c}{ Source } & df & Sum Squares & F Ratio & P Value \\
\hline Retention & 1 & 0.05 & 0.04 & 0.8394 \\
Sex & 1 & 22.57 & 18.41 & $<0.0001$ \\
Time & 1 & 2.05 & 1.68 & 0.1974 \\
Retention-x-Sex & 1 & 0.18 & 0.15 & 0.7038 \\
Retention-x-Time & 1 & 0.11 & 0.09 & 0.7697 \\
Sex-x-Time & 1 & 0.00 & 0.00 & 0.9911 \\
Retention-x-Sex-x-Time & 1 & 0.07 & 0.06 & 0.8112 \\
\hline
\end{tabular}

Table C-23. Results of ANCOVA tests of whether the amount of relapse depends on the kind of retention; dependent variable is ANB, with time-out-of-treatment as a covariate.

\begin{tabular}{lcccc}
\hline \multicolumn{1}{c}{ Source } & df & Sum Squares & F Ratio & P Value \\
\hline Retention & 1 & 0.08 & 0.06 & 0.8067 \\
Sex & 1 & 31.02 & 22.28 & $<0.0001$ \\
Time & 1 & 0.66 & 0.48 & 0.4907 \\
Retention-x-Sex & 1 & 0.47 & 0.34 & 0.5607 \\
Retention-x-Time & 1 & 0.13 & 0.09 & 0.7626 \\
Sex-x-Time & 1 & 2.80 & 2.01 & 0.1581 \\
Retention-x-Sex-x-Time & 1 & 0.71 & 0.51 & 0.4753 \\
\hline
\end{tabular}

Table C-24. Results of ANCOVA tests of whether the amount of relapse depends on the kind of retention; dependent variable is AOBO (Wits appraisal), with time-out-of-treatment as a covariate.

\begin{tabular}{lcccc}
\hline \multicolumn{1}{c}{ Source } & df & Sum Squares & F Ratio & P Value \\
\hline Retention & 1 & 2.82 & 0.83 & 0.3626 \\
Sex & 1 & 23.27 & 6.87 & 0.0096 \\
Time & 1 & 1.61 & 0.48 & 0.4917 \\
Retention-x-Sex & 1 & 3.04 & 0.90 & 0.3449 \\
Retention-x-Time & 1 & 3.34 & 0.99 & 0.3223 \\
Sex-x-Time & 1 & 12.14 & 3.58 & 0.0602 \\
Retention-x-Sex-x-Time & 1 & 0.32 & 0.09 & 0.7594 \\
\hline
\end{tabular}


Table C-25. Results of ANCOVA tests of whether the amount of relapse depends on the kind of retention; dependent variable is Downs' Occlusal Plane (DOP), with time-out-of-treatment as a covariate.

\begin{tabular}{lcccc}
\hline \multicolumn{1}{c}{ Source } & df & Sum Squares & F Ratio & P Value \\
\hline Retention & 1 & 0.97 & 0.21 & 0.6450 \\
Sex & 1 & 41.60 & 9.14 & 0.0029 \\
Time & 1 & 0.04 & 0.01 & 0.9224 \\
Retention-x-Sex & 1 & 1.48 & 0.32 & 0.5699 \\
Retention-x-Time & 1 & 2.03 & 0.45 & 0.5051 \\
Sex-x-Time & 1 & 0.02 & 0.01 & 0.9415 \\
Retention-x-Sex-x-Time & 1 & 0.75 & 0.16 & 0.6859 \\
\hline
\end{tabular}

Table C-26. Results of ANCOVA tests of whether the amount of relapse depends on the kind of retention; dependent variable is $\mathrm{Z}$ angle, with time-out-oftreatment as a covariate.

\begin{tabular}{lcccc}
\hline \multicolumn{1}{c}{ Source } & df & Sum Squares & F Ratio & P Value \\
\hline Retention & 1 & 0.03 & 0.00 & 0.9670 \\
Sex & 1 & 276.53 & 16.70 & $<0.0001$ \\
Time & 1 & 26.83 & 1.62 & 0.2049 \\
Retention-x-Sex & 1 & 10.59 & 0.64 & 0.4250 \\
Retention-x-Time & 1 & 30.57 & 1.85 & 0.1761 \\
Sex-x-Time & 1 & 19.98 & 1.21 & 0.2737 \\
Retention-x-Sex-x-Time & 1 & 5.70 & 0.34 & 0.5583 \\
\hline
\end{tabular}

Table C-27. Results of ANCOVA tests of whether the amount of relapse depends on the kind of retention; dependent variable is Upper Lip (UL), with time-out-oftreatment as a covariate.

\begin{tabular}{lcccc}
\hline \multicolumn{1}{c}{ Source } & df & Sum Squares & F Ratio & P Value \\
\hline Retention & 1 & 0.72 & 0.18 & 0.6701 \\
Sex & 1 & 2.04 & 0.51 & 0.4744 \\
Time & 1 & 3.73 & 0.94 & 0.3333 \\
Retention-x-Sex & 1 & 2.35 & 0.59 & 0.4425 \\
Retention-x-Time & 1 & 0.03 & 0.01 & 0.9348 \\
Sex-x-Time & 1 & 0.99 & 0.25 & 0.6169 \\
Retention-x-Sex-x-Time & 1 & 0.05 & 0.01 & 0.9119 \\
\hline
\end{tabular}


Table C-28. Results of ANCOVA tests of whether the amount of relapse depends on the kind of retention; dependent variable is Total Chin (TC), with time-out-oftreatment as a covariate.

\begin{tabular}{lccrr}
\hline \multicolumn{1}{c}{ Source } & df & Sum Squares & F Ratio & P Value \\
\hline Retention & 1 & 2.75 & 1.01 & 0.3169 \\
Sex & 1 & 57.74 & 21.15 & $<0.0001$ \\
Time & 1 & 24.24 & 8.88 & 0.0033 \\
Retention-x-Sex & 1 & 2.75 & 1.01 & 0.3169 \\
Retention-x-Time & 1 & 1.32 & 0.48 & 0.4879 \\
Sex-x-Time & 1 & 2.93 & 1.08 & 0.3014 \\
Retention-x-Sex-x-Time & 1 & 4.99 & 1.83 & 0.1784 \\
\hline
\end{tabular}

Table C-29. Results of ANCOVA tests of whether the amount of relapse depends on the kind of retention; dependent variable is Anterior Facial Height (AFH), with time-out-of-treatment as a covariate.

\begin{tabular}{lcccc}
\hline \multicolumn{1}{c}{ Source } & df & Sum Squares & F Ratio & P Value \\
\hline Retention & 1 & 8.12 & 1.75 & 0.1878 \\
Sex & 1 & 67.12 & 14.46 & 0.0002 \\
Time & 1 & 13.95 & 3.00 & 0.0850 \\
Retention-x-Sex & 1 & 20.44 & 4.40 & 0.0375 \\
Retention-x-Time & 1 & 0.57 & 0.12 & 0.7262 \\
Sex-x-Time & 1 & 0.25 & 0.05 & 0.8183 \\
Retention-x-Sex-x-Time & 1 & 1.95 & 0.42 & 0.5175 \\
\hline
\end{tabular}

Table C-30. Results of ANCOVA tests of whether the amount of relapse depends on the kind of retention; dependent variable is Posterior Facial Height (PFH), with time-out-of-treatment as a covariate.

\begin{tabular}{lccrc}
\hline \multicolumn{1}{c}{ Source } & df & Sum Squares & F Ratio & P Value \\
\hline Retention & 1 & 16.41 & 2.31 & 0.1302 \\
Sex & 1 & 373.39 & 52.66 & $<0.0001$ \\
Time & 1 & 4.73 & 0.67 & 0.4155 \\
Retention-x-Sex & 1 & 17.06 & 2.41 & 0.1228 \\
Retention-x-Time & 1 & 5.18 & 0.73 & 0.3942 \\
Sex-x-Time & 1 & 5.48 & 0.77 & 0.3805 \\
Retention-x-Sex-x-Time & 1 & 1.36 & 0.19 & 0.6625 \\
\hline
\end{tabular}


Table C-31. Results of ANCOVA tests of whether the amount of relapse depends on the kind of retention; dependent variable is Overbite $(\mathrm{OB})$, with time-out-oftreatment as a covariate.

\begin{tabular}{lcccc}
\hline \multicolumn{1}{c}{ Source } & df & Sum Squares & F Ratio & P Value \\
\hline Retention & 1 & 3.68 & 2.18 & 0.1417 \\
Sex & 1 & 0.34 & 0.20 & 0.6541 \\
Time & 1 & 1.71 & 1.01 & 0.3156 \\
Retention-x-Sex & 1 & 0.08 & 0.05 & 0.8252 \\
Retention-x-Time & 1 & 0.81 & 0.48 & 0.4900 \\
Sex-x-Time & 1 & 0.01 & 0.01 & 0.9349 \\
Retention-x-Sex-x-Time & 1 & 0.39 & 0.23 & 0.6304 \\
\hline
\end{tabular}

Table C-32. Results of ANCOVA tests of whether the amount of relapse depends on the kind of retention; dependent variable is Overjet (OJ), with time-out-oftreatment as a covariate.

\begin{tabular}{lcccc}
\hline \multicolumn{1}{c}{ Source } & df & Sum Squares & F Ratio & P Value \\
\hline Retention & 1 & 0.03 & 0.03 & 0.8660 \\
Sex & 1 & 0.06 & 0.05 & 0.8293 \\
Time & 1 & 0.00 & 0.00 & 0.9957 \\
Retention-x-Sex & 1 & 0.46 & 0.37 & 0.5419 \\
Retention-x-Time & 1 & 1.26 & 1.03 & 0.3127 \\
Sex-x-Time & 1 & 0.40 & 0.32 & 0.5703 \\
Retention-x-Sex-x-Time & 1 & 0.02 & 0.02 & 0.8995 \\
\hline
\end{tabular}


APPENDIX D. DESCRIPTIVE STATISTICS FOR THOSE VARIABLES EXHIBITING SIGNIFICANCE FOR SEXUAL DIMORPHISM 
Table D-1. Descriptive statistics, by sex, for those variables that achieved statistical significant at the pretreatment examination.

\begin{tabular}{|c|c|c|c|c|c|c|c|c|}
\hline \multirow[b]{2}{*}{ Variable } & \multicolumn{3}{|c|}{ Males } & \multicolumn{3}{|c|}{ Females } & \multirow[b]{2}{*}{$\mathrm{F}$} & \multirow[b]{2}{*}{$\mathrm{P}$} \\
\hline & $\mathrm{n}$ & $\bar{x}$ & sem & $\mathrm{n}$ & $\bar{x}$ & sem & & \\
\hline COS & 43 & 2.56 & 0.13 & 123 & 2.05 & 0.08 & 11.85 & 0.0007 \\
\hline $\mathrm{MC}$ & 43 & 8.14 & 0.73 & 123 & 5.84 & 0.43 & 7.34 & 0.0075 \\
\hline ASR & 43 & 35.13 & 0.81 & 123 & 32.74 & 0.48 & 6.53 & 0.0115 \\
\hline ISR & 43 & 18.85 & 1.05 & 123 & 15.21 & 0.62 & 8.94 & 0.0032 \\
\hline IAR & 43 & 80.37 & 2.48 & 123 & 72.51 & 1.47 & 7.42 & 0.0072 \\
\hline ASP & 43 & 36.17 & 0.44 & 123 & 34.38 & 0.26 & 12.29 & 0.0006 \\
\hline UMW & 43 & 52.03 & 0.49 & 123 & 50.26 & 0.29 & 9.54 & 0.0024 \\
\hline LMW & 43 & 51.25 & 0.42 & 123 & 50.08 & 0.25 & 5.75 & 0.0176 \\
\hline ISD & 43 & 3.99 & 0.22 & 123 & 3.14 & 0.13 & 11.55 & 0.0009 \\
\hline ASD & 43 & 9.63 & 0.22 & 123 & 8.76 & 0.13 & 11.54 & 0.0009 \\
\hline ID & 43 & 27.13 & 1.12 & 123 & 22.09 & 0.66 & 14.99 & 0.0002 \\
\hline ANB & 43 & 5.35 & 0.35 & 123 & 4.16 & 0.20 & 8.73 & 0.0036 \\
\hline AOBO & 43 & 4.74 & 0.55 & 123 & 1.47 & 0.33 & 26.09 & $<0.0001$ \\
\hline DOP & 43 & 7.84 & 0.59 & 123 & 9.50 & 0.35 & 5.78 & 0.0174 \\
\hline $\mathrm{Z}$ angle & 43 & 64.88 & 1.31 & 123 & 70.29 & 0.78 & 12.61 & 0.0005 \\
\hline OB & 43 & 5.42 & 0.33 & 123 & 3.59 & 0.19 & 23.53 & $<0.0001$ \\
\hline OJ & 43 & 7.16 & 0.44 & 123 & 4.98 & 0.26 & 18.57 & $<0.0001$ \\
\hline
\end{tabular}


Table D-2. Descriptive statistics, by sex, for those variables that achieved statistical significant at the posttreatment examination.

\begin{tabular}{|c|c|c|c|c|c|c|c|c|}
\hline \multirow[b]{2}{*}{ Variable } & \multicolumn{3}{|c|}{ Males } & \multicolumn{3}{|c|}{ Females } & \multirow[b]{2}{*}{ F } & \multirow[b]{2}{*}{$\mathrm{P}$} \\
\hline & $\mathrm{n}$ & $\bar{x}$ & sem & $\mathrm{n}$ & $\bar{x}$ & sem & & \\
\hline ASP & 43 & 38.23 & 0.36 & 123 & 36.90 & 0.21 & 10.16 & 0.0017 \\
\hline UMW & 43 & 51.92 & 0.43 & 123 & 50.29 & 0.26 & 10.46 & 0.0015 \\
\hline LMW & 43 & 49.47 & 0.44 & 123 & 48.03 & 0.26 & 7.99 & 0.0053 \\
\hline UCW & 43 & 34.24 & 0.29 & 123 & 33.06 & 0.17 & 12.52 & 0.0005 \\
\hline ASD & 43 & 10.21 & 0.16 & 123 & 9.66 & 0.10 & 8.65 & 0.0037 \\
\hline ID & 43 & 24.73 & 1.11 & 123 & 20.60 & 0.65 & 10.34 & 0.0016 \\
\hline ASW & 43 & 29.01 & 0.24 & 123 & 28.35 & 0.14 & 5.59 & 0.0192 \\
\hline DOP & 43 & 8.77 & 0.59 & 123 & 10.19 & 0.35 & 4.36 & 0.0384 \\
\hline UL & 43 & 17.37 & 0.40 & 123 & 14.40 & 0.23 & 41.87 & $<0.0001$ \\
\hline TC & 43 & 16.95 & 0.35 & 123 & 15.39 & 0.21 & 14.83 & 0.0002 \\
\hline AFH & 43 & 70.16 & 0.81 & 123 & 66.77 & 0.48 & 13.00 & 0.0004 \\
\hline PFH & 43 & 54.23 & 0.71 & 123 & 50.38 & 0.42 & 22.03 & $<0.0001$ \\
\hline
\end{tabular}


Table D-3. Descriptive statistics, by sex, for those variables that achieved statistical significant at the recall examination.

\begin{tabular}{|c|c|c|c|c|c|c|c|c|}
\hline \multirow[b]{2}{*}{ Variable } & \multicolumn{3}{|c|}{ Males } & \multicolumn{3}{|c|}{ Females } & \multirow[b]{2}{*}{$\mathrm{F}$} & \multirow[b]{2}{*}{ P } \\
\hline & $\mathrm{n}$ & $\bar{x}$ & sem & $\mathrm{n}$ & $\bar{x}$ & sem & & \\
\hline IAR & 43 & 84.60 & 2.26 & 123 & 78.54 & 1.34 & 5.32 & 0.0223 \\
\hline ASP & 43 & 36.64 & 0.37 & 123 & 35.24 & 0.22 & 10.40 & 0.0015 \\
\hline UMW & 43 & 51.64 & 0.45 & 123 & 49.51 & 0.27 & 16.20 & $<0.0001$ \\
\hline LMW & 43 & 49.44 & 0.47 & 123 & 47.47 & 0.28 & 13.07 & 0.0004 \\
\hline UCW & 43 & 34.24 & 0.30 & 123 & 32.40 & 0.17 & 28.70 & $<0.0001$ \\
\hline LCW & 43 & 25.25 & 0.25 & 123 & 24.25 & 0.15 & 12.09 & 0.0006 \\
\hline ID & 43 & 24.52 & 1.10 & 123 & 20.56 & 0.65 & 9.52 & 0.0024 \\
\hline ASW & 43 & 28.13 & 0.25 & 123 & 27.19 & 0.15 & 10.90 & 0.0012 \\
\hline FMIA & 43 & 66.72 & 0.98 & 123 & 63.13 & 0.58 & 9.96 & 0.0019 \\
\hline FMA & 43 & 23.70 & 1.01 & 123 & 26.59 & 0.60 & 6.04 & 0.0151 \\
\hline ANB & 43 & 1.81 & 0.29 & 123 & 3.28 & 0.17 & 19.63 & $<0.0001$ \\
\hline DOP & 43 & 6.37 & 0.65 & 123 & 8.98 & 0.38 & 12.06 & 0.0007 \\
\hline UL & 43 & 15.60 & 0.36 & 123 & 12.37 & 0.21 & 60.39 & $<0.0001$ \\
\hline TC & 43 & 19.14 & 0.39 & 123 & 16.20 & 0.23 & 41.54 & $<0.0001$ \\
\hline AFH & 43 & 72.60 & 0.84 & 123 & 67.72 & 0.50 & 24.76 & $<0.0001$ \\
\hline PFH & 43 & 58.58 & 0.72 & 123 & 51.26 & 0.43 & 75.57 & $<0.0001$ \\
\hline
\end{tabular}


Table D-4. Descriptive statistics, by sex, for those variables that achieved statistical significant for the in-treatment changes.

\begin{tabular}{|c|c|c|c|c|c|c|c|c|}
\hline \multirow[b]{2}{*}{ Variable } & \multicolumn{3}{|c|}{ Males } & \multicolumn{3}{|c|}{ Females } & \multirow[b]{2}{*}{ F } & \multirow[b]{2}{*}{$\mathrm{P}$} \\
\hline & $\mathrm{n}$ & $\bar{x}$ & sem & $\mathrm{n}$ & $\bar{x}$ & sem & & \\
\hline COS & 43 & -1.97 & 0.15 & 123 & -1.49 & 0.09 & 7.36 & 0.0074 \\
\hline $\mathrm{MC}$ & 43 & -7.09 & 0.75 & 123 & -4.59 & 0.44 & 8.23 & 0.0047 \\
\hline ISR & 43 & -1.20 & 1.04 & 123 & 2.24 & 0.61 & 8.16 & 0.0048 \\
\hline ISD & 43 & -0.11 & 0.21 & 123 & 0.62 & 0.13 & 8.53 & 0.0040 \\
\hline ID & 43 & -2.41 & 0.38 & 123 & -1.49 & 0.23 & 4.30 & 0.0397 \\
\hline FMIA & 43 & 4.30 & 0.91 & 123 & 1.60 & 0.54 & 6.56 & 0.0113 \\
\hline SNA & 43 & -2.58 & 0.25 & 123 & -1.59 & 0.15 & 11.31 & 0.0010 \\
\hline SNB & 43 & 0.35 & 0.22 & 123 & -0.31 & 0.13 & 6.73 & 0.0103 \\
\hline ANB & 43 & -2.93 & 0.27 & 123 & -1.28 & 0.16 & 28.17 & $<0.0001$ \\
\hline АOBO & 43 & -3.67 & 0.45 & 123 & -1.20 & 0.26 & 22.74 & $<0.0001$ \\
\hline $\mathrm{Z}$ angle & 43 & 9.53 & 0.93 & 123 & 5.65 & 0.55 & 12.97 & 0.0004 \\
\hline UL & 43 & 3.60 & 0.36 & 123 & 1.37 & 0.21 & 28.63 & $<0.0001$ \\
\hline TC & 43 & 2.42 & 0.27 & 123 & 1.42 & 0.16 & 10.03 & 0.0018 \\
\hline $\mathrm{AFH}$ & 43 & 5.72 & 0.41 & 123 & 3.19 & 0.24 & 28.16 & $<0.0001$ \\
\hline PFH & 43 & 6.42 & 0.52 & 123 & 3.24 & 0.31 & 27.65 & $<0.0001$ \\
\hline $\mathrm{OB}$ & 43 & -3.28 & 0.33 & 123 & -1.35 & 0.20 & 25.24 & $<0.0001$ \\
\hline OJ & 43 & -4.65 & 0.44 & 123 & -2.54 & 0.26 & 17.43 & $<0.0001$ \\
\hline
\end{tabular}


Table D-5. Descriptive statistics, by sex, for those variables that achieved statistical significant at the posttreatment changes.

\begin{tabular}{|c|c|c|c|c|c|c|c|c|}
\hline \multirow[b]{2}{*}{ Variable } & \multicolumn{3}{|c|}{ Males } & \multicolumn{3}{|c|}{ Females } & \multirow[b]{2}{*}{$\mathrm{F}$} & \multirow[b]{2}{*}{ P } \\
\hline & $\mathrm{n}$ & $\bar{x}$ & sem & $\mathrm{n}$ & $\bar{x}$ & sem & & \\
\hline UMW & 43 & -0.28 & 0.14 & 123 & -0.77 & 0.08 & 8.98 & 0.0031 \\
\hline LMW & 43 & -0.02 & 0.16 & 123 & -0.55 & 0.10 & 7.95 & 0.0054 \\
\hline UCW & 43 & 0.00 & 0.17 & 123 & -0.65 & 0.10 & 10.96 & 0.0011 \\
\hline LCW & 43 & -0.89 & 0.16 & 123 & -1.29 & 0.09 & 4.81 & 0.0297 \\
\hline FMIA & 43 & 2.95 & 0.58 & 123 & 1.04 & 0.34 & 7.97 & 0.0053 \\
\hline FMA & 43 & -1.98 & 0.29 & 123 & -0.31 & 0.17 & 23.70 & $<0.0001$ \\
\hline SNB & 43 & 0.56 & 0.17 & 123 & -0.30 & 0.10 & 19.62 & $<0.0001$ \\
\hline ANB & 43 & -0.60 & 0.18 & 123 & 0.40 & 0.11 & 23.29 & $<0.0001$ \\
\hline $\mathrm{AOBO}$ & 43 & 0.53 & 0.28 & 123 & 1.41 & 0.17 & 7.32 & 0.0075 \\
\hline DOP & 43 & -2.40 & 0.32 & 123 & -1.21 & 0.19 & 10.13 & 0.0017 \\
\hline $\mathrm{Z}$ angle & 43 & 5.72 & 0.62 & 123 & 2.72 & 0.37 & 17.44 & $<0.0001$ \\
\hline TC & 43 & 2.19 & 0.26 & 123 & 0.80 & 0.15 & 21.40 & $<0.0001$ \\
\hline $\mathrm{AFH}$ & 43 & 2.44 & 0.33 & 123 & 0.95 & 0.20 & 14.96 & 0.0002 \\
\hline PFH & 43 & 4.35 & 0.41 & 123 & 0.88 & 0.24 & 54.22 & $<0.0001$ \\
\hline
\end{tabular}


APPENDIX E. EXTRACTION PATTERNS 
Table E-1. Two-way ANOVA tests evaluating whether the amount of posttreatment relapse depends on the extraction pattern, while controlling for type of retention. ${ }^{1}$

\begin{tabular}{|c|c|c|c|c|c|c|c|c|c|}
\hline \multirow[b]{2}{*}{ Variable } & \multicolumn{3}{|c|}{ Extraction Pattern } & \multicolumn{3}{|c|}{ Retention } & \multicolumn{3}{|c|}{ Interaction } \\
\hline & df & F Ratio & P Value & df & F Ratio & P Value & $\mathrm{df}$ & F Ratio & P Value \\
\hline ADisc & 2 & 1.43 & 0.2423 & 1 & 3.49 & 0.0640 & 2 & 0.72 & 0.4877 \\
\hline MADisc & 2 & 0.51 & 0.6032 & 1 & 0.04 & 0.8419 & 2 & 0.09 & 0.9171 \\
\hline $\cos$ & 2 & 1.80 & 0.1696 & 1 & 1.26 & 0.2641 & 2 & 0.52 & 0.5928 \\
\hline MC & 2 & 3.25 & 0.0420 & 1 & 0.20 & 0.6546 & 2 & 0.09 & 0.9115 \\
\hline ASR & 2 & 2.29 & 0.1053 & 1 & 2.68 & 0.1039 & 2 & 0.20 & 0.8188 \\
\hline ISR & 2 & 2.04 & 0.1339 & 1 & 5.96 & 0.0159 & 2 & 0.20 & 0.8151 \\
\hline IAR & 2 & 1.16 & 0.3156 & 1 & 1.30 & 0.2556 & 2 & 1.54 & 0.2183 \\
\hline ASP & 2 & 2.78 & 0.0654 & 1 & 0.02 & 0.8833 & 2 & 0.83 & 0.4369 \\
\hline UMW & 2 & 1.07 & 0.3453 & 1 & 3.76 & 0.0545 & 2 & 0.65 & 0.5248 \\
\hline LMW & 2 & 1.01 & 0.3675 & 1 & 0.74 & 0.3904 & 2 & 0.29 & 0.7481 \\
\hline UCW & 2 & 3.17 & 0.0450 & 1 & 0.08 & 0.7724 & 2 & 2.30 & 0.1046 \\
\hline LCW & 2 & 1.70 & 0.1863 & 1 & 4.28 & 0.0404 & 2 & 0.95 & 0.3896 \\
\hline ISD & 2 & 1.53 & 0.2204 & 1 & 3.98 & 0.0481 & 2 & 0.12 & 0.8897 \\
\hline ASD & 2 & 1.97 & 0.1429 & 1 & 1.53 & 0.2178 & 2 & 0.11 & 0.8935 \\
\hline ID & 2 & 0.09 & 0.9177 & 1 & 0.02 & 0.8970 & 2 & 0.49 & 0.6156 \\
\hline ASW & 2 & 4.37 & 0.0145 & 1 & 2.35 & 0.1280 & 2 & 1.48 & 0.2319 \\
\hline II & 2 & 0.43 & 0.6507 & 1 & 1.58 & 0.2104 & 2 & 0.71 & 0.4920 \\
\hline FMIA & 2 & 0.16 & 0.8544 & 1 & 0.14 & 0.7045 & 2 & 0.48 & 0.6225 \\
\hline FMA & 2 & 1.28 & 0.2825 & 1 & 0.95 & 0.3309 & 2 & 0.37 & 0.6894 \\
\hline IMPA & 2 & 0.32 & 0.7279 & 1 & 1.00 & 0.3201 & 2 & 0.27 & 0.7613 \\
\hline SNA & 2 & 0.03 & 0.9737 & 1 & 0.51 & 0.4756 & 2 & 1.64 & 0.1973 \\
\hline SNB & 2 & 0.06 & 0.9385 & 1 & 0.32 & 0.5719 & 2 & 0.85 & 0.4279 \\
\hline
\end{tabular}


Table E-1. Continued.

\begin{tabular}{|c|c|c|c|c|c|c|c|c|c|}
\hline \multirow[b]{2}{*}{ Variable } & \multicolumn{3}{|c|}{ Extraction Pattern } & \multicolumn{3}{|c|}{ Retention } & \multicolumn{3}{|c|}{ Interaction } \\
\hline & $\mathrm{df}$ & F Ratio & P Value & $\mathrm{df}$ & F Ratio & P Value & $\mathrm{df}$ & F Ratio & P Value \\
\hline ANB & 2 & 0.10 & 0.9041 & 1 & 0.04 & 0.8499 & 2 & 1.09 & 0.3398 \\
\hline $\mathrm{AOBO}$ & 2 & 1.33 & 0.2685 & 1 & 0.19 & 0.6640 & 2 & 1.65 & 0.1968 \\
\hline DOP & 2 & 0.70 & 0.4960 & 1 & 1.13 & 0.2894 & 2 & 1.42 & 0.2454 \\
\hline Z angle & 2 & 0.92 & 0.4014 & 1 & 0.01 & 0.9432 & 2 & 0.16 & 0.8507 \\
\hline UL & 2 & 0.78 & 0.4607 & 1 & 0.06 & 0.8106 & 2 & 0.79 & 0.4554 \\
\hline TC & 2 & 1.31 & 0.2733 & 1 & 1.19 & 0.2765 & 2 & 1.49 & 0.2281 \\
\hline AFH & 2 & 1.68 & 0.1895 & 1 & 0.18 & 0.6720 & 2 & 0.36 & 0.6955 \\
\hline PFH & 2 & 1.88 & 0.1566 & 1 & 0.03 & 0.8542 & 2 & 0.67 & 0.5127 \\
\hline OB & 2 & 2.09 & 0.1280 & 1 & 0.03 & 0.8610 & 2 & 2.11 & 0.1255 \\
\hline OJ & 2 & 1.08 & 0.3414 & 1 & 0.20 & 0.6554 & 2 & 0.32 & 0.7237 \\
\hline
\end{tabular}

1The three extraction patterns are non-extraction, 4-4/4-4, and 4-4/5-5; the two types of retention are upper

Hawley retainer with fixed lower 3-3 or upper and lower Hawley retainers. 


\section{VITA}

Mark Owens was born on January 11, 1977 in Memphis, TN. Mark graduated from White Station High School in 1995. He attended Middle Tennessee State University and graduated with a Bachelor's Degree in Biology in 2000. Mark received his Doctor of Dental Surgery degree in May 2005 from the University of the Tennessee Health Science Center. He then spent the next three years in private dental practice in Shelbyville, TN, before returning to Memphis to become a graduate student in the Department of Orthodontics at the University of Tennessee Health Science Center in 2008. Mark received his Master of Dental Science in May 2011. 